\title{
PHOTOBIOREACTOR DESIGN FOR IMPROVED ENERGY EFFICIENCY OF MICROALGAE PRODUCTION
}

\author{
A Thesis \\ Presented to \\ the Faculty of \\ California Polytechnic State University, \\ San Luis Obispo
}

\author{
In Partial Fulfillment \\ of the Requirements for the Degree \\ Master of Science in Biochemical Engineering
}

by

Alexander Burns

December 2014 
(C) 2014

Alexander Burns

ALL RIGHTS RESERVED 
TITLE:

AUTHOR:

DATE SUBMITTED:

COMMITTEE CHAIR:

COMMITTEE MEMBER:

COMMITTEE MEMBER:
Photobioreactor design for improved energy efficiency of microalgae production

Alexander Burns

December 2014

Yarrow Nelson, PhD

Professor of Civil \& Environmental Engineering

Brian Hampson, $\mathrm{PhD}$

Professor of Food Science \& Nutrition

Tryg Lundquist, $\mathrm{PhD}$

Professor of Civil \& Environmental Engineering 


\begin{abstract}
Photobioreactor Design for Improved Energy Efficiency of Microalgae Production
\end{abstract}

\begin{abstract}
Alexander Burns
The objective of this research was to investigate a new photobioreactor (PBR) design for microalgae production that retains the typical advantages of existing tubular PBRs while reducing power consumption by providing simultaneous culture circulation and gas exchange with airlift alone and no centrifugal recirculating pump. Traditional tubular PBR designs feature a compressed air supply and a centrifugal pump for culture circulation and gas exchange. Circulation and gas exchange in a closed-system PBR is necessary to keep the algae suspended and to provide sufficient mass transfer (mainly for the exchange of oxygen and carbon dioxide). In a traditional tubular PBR sparged air keeps the culture well mixed and strips out excess dissolved oxygen in an airlift-column unit, while the centrifugal pump circulates the culture in the tubular stage and decreases the amount of air bubbles traveling into this stage; where most of the photosynthesis occurs. The PBR design proposed herein does away with the usual centrifugal pump. The air blower performs both gas exchange in the airlift columns and system-wide circulation. This builds on a previous tubular PBR design that provides circulation and gas exchange by airlift alone, which was patented by Cathcart in 2011. However, the Cathcart patent does not provide data on mixing, gas exchange, energy consumption, flow regime or biomass productivity. The new design described here builds on the Cathcart design, but includes several unique design features, such as larger diffuser columns which provide airlift-induced flow for a series of vertical PBR tubes. To perform a power consumption
\end{abstract}


analysis, a pilot-scale prototype of the new PBR design was built and operated. The prototype PBR consisted of two airlift columns attached to $9 \mathrm{~m}$ of vertical serpentine tubing connected to the top and bottom by standard 90-degree PVC elbows in a U-bend fashion to each column to make a total working volume of $235 \mathrm{~L}$. The airlift columns were about $1.5 \mathrm{~m}$ tall and $30.5 \mathrm{~cm}$ ID, while the serpentine tubes were about $0.9 \mathrm{~m}$ tall and $7.6 \mathrm{~cm}$ ID to make a total of five vertical tubes for every airlift column. Data collected for this prototype design suggest an average overall areal productivity (OAP) of $111 \mathrm{~g} \mathrm{~m}^{-2} \mathrm{~d}^{-1}$ ( $\mathrm{g}$ biomass $\mathrm{m}^{-2}$ total land area with empty space day ${ }^{-1}$ ), an average illuminated surface productivity (ISP) of $14.3 \mathrm{~g} \mathrm{~m}^{-2} \mathrm{~d}^{-1}$ ( $\mathrm{g}$ biomass $\mathrm{m}^{-2}$ reactor photo-stage day $^{-1}$ ), an average volumetric productivity (VP) of $0.55 \mathrm{~g} \mathrm{~L}^{-1} \mathrm{~d}^{-1}$ ( $\mathrm{g}$ biomass $\mathrm{L}^{-1}$ reactor working volume day ${ }^{-1}$ ), a specific power input in the range of 330 to $360 \mathrm{~W} \mathrm{~m}^{-3}$ (W power needed for culture circulation and gas exchange $\mathrm{m}^{-3}$ reactor working volume) and a specific biomass productivity (SBP) in the range of 17.6 to $19.1 \mathrm{mg} \mathrm{kJ}^{-1}$ (mg biomass $\mathrm{kJ}^{-1}$ energy needed for culture circulation and gas exchange) with Chlorella vulgaris as the model algae. The biomass productivity per energy input (SBP) of the new PBR design appears to be higher than that of similar designs currently described in the literature. Elimination of the centrifugal pump in a tubular PBR design is a concept worth further study for potential energy savings. 


\section{ACKNOWLEDGMENTS}

I am indebted to Dr. Yarrow Nelson for his role as an advisor over my past two years at Cal Poly. His experience and feedback have been invaluable in the development of this thesis, and his good humor and ingenuity have pulled me out of many project issues. I would like to thank Dr. Brian Hampson for his inspiration, hard work and friendship throughout my time at Cal Poly. Thank you both for the dozens of meetings, hundreds of laughs, providing me this great opportunity and for all your help. Please keep your high standards and amazing ways of energizing people. Thanks to Dr. Tryg Lundquist for his role on my thesis committee and his endless knowledge of algae. I would also like to thank my co-worker Andrew Balberchak for being such a great project partner; his enthusiasm and passion for solving problems and building things made the completion of this thesis possible. Thank you Andrew for the countless hours of hard work, sweat and blood spent in the machine shops to make the proposed photobioreactor design a reality.

I would like to thank my friends here at Cal Poly: Alex Wilk, Jacob Suvorov, Madalyn Hunt, Rebecca Salcedo, and Tyler Dunaway for their enthusiasm, support, and constant belief in me throughout my time at Cal Poly. Being around all of them gave me the best college experience I could have asked for at Cal Poly.

Finally, I would like to thank my parents for encouraging me and teaching me how important a valuable education can be. I am truly grateful to have two parents who have sacrificed their time and energy for the benefit of their children. Without their continued support, love and encouragement I would not have been able to have the drive to pursue both a Bachelor's and Master's Degree in Biochemical Engineering.

Funding for this project was provided by the Center for Applications in Biotechnology $(\mathrm{CAB})$ at California Polytechnic State University San Luis Obispo. 


\section{TABLE OF CONTENTS}

\section{Page}

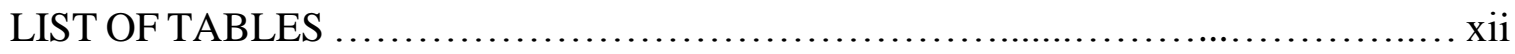

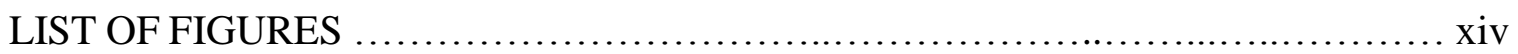

\section{CHAPTER}

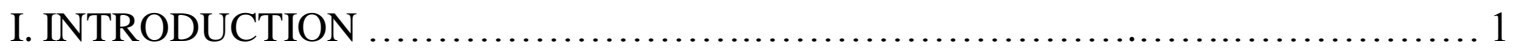

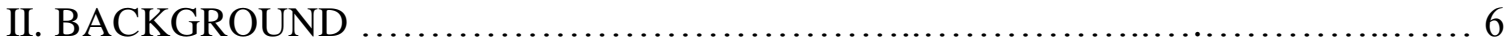

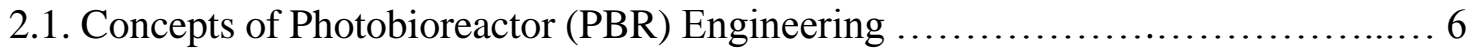

2.2. A Brief History of Microalgae Cultivation Technology Development ............ 7

2.2.1. Technological Developments from the Early 1950s to the $1990 \mathrm{~s}$.............. 7

2.2.2. Attempts of Large-Scale PBR Microalgae Facilities in the $1990 \mathrm{~s}$............. 9

2.2.2.1. Sagdiana PGT (Tadshikistan) ................................... 9

2.2.2.2. Photo Bioreactors Ltd (Spain) .................................. 10

2.2.2.3. Hidrobiologica SA (Argentina) .................................. 11

2.2.2.4. Inalco S.p.A. (Italy) .......................................... 12

2.2.2.5. Microalgae S.p.A. (Italy) ..................................... 12

2.3. Cultivation Conditions for Microalgae ................................... 13

2.3.1. Phototrophic Cultivation ......................................... 13

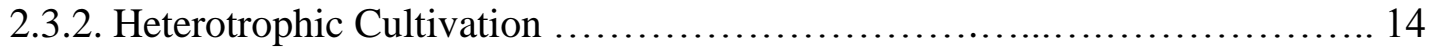

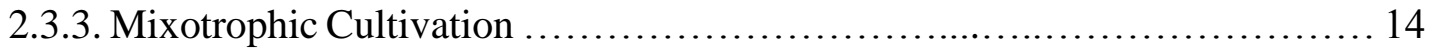

2.3.4. Photoheterotrophic Cultivation ................................... 14

2.3.5. Comparison of Different Cultivation Conditions ......................... 15 


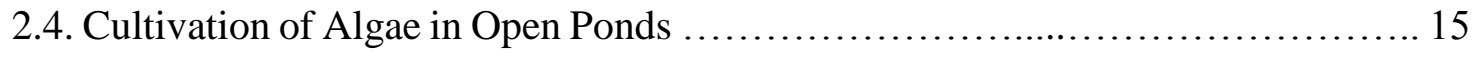

2.4.1. Lakes and Natural Ponds ................................................. 16

2.4.2. Circular Ponds ............................................................ 16

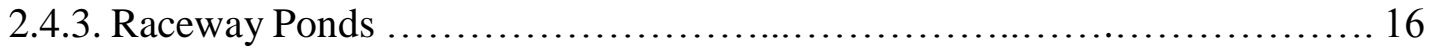

2.5. Comparison of PBRs to Open Ponds ........................................ 17

2.5.1. Pros and Cons of PBRs and Open Ponds .................................... 17

2.5.2. Coupling Ponds and Photobioreactors .................................. 19

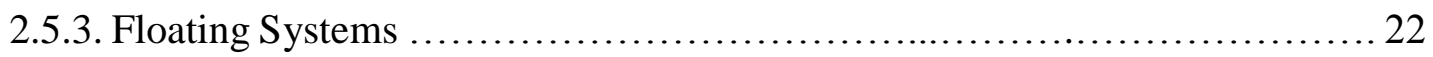

2.5.4. Summary of Present Enterprises ......................................... 23

2.6. Design Criteria for Photobioreactors ......................................... 28

2.6.1. Surface-to-Volume Ratio (S/V) ..................................... 28

2.6.2. Orientation and Inclination ............................................. 28

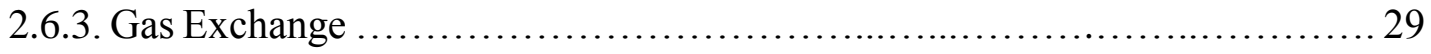

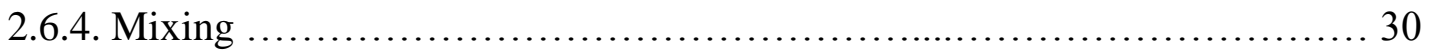

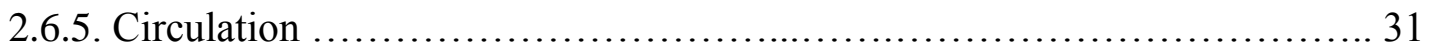

2.6.6. Temperature control ......................................................... 32

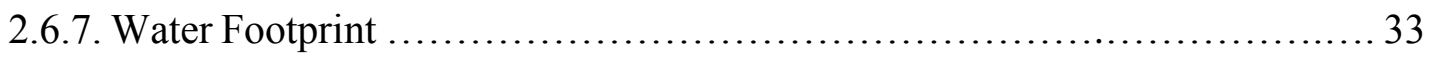

2.6.8. Construction Materials for PBRs ........................................... 33

2.7. Scale Up of Photobioreactors ...............................................

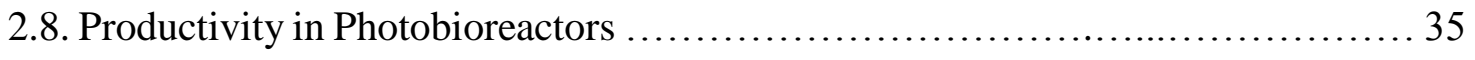

2.8.1. Methods of Evaluation and Comparison .................................. 35

2.9. Types of PBRs and Early Attempts at Cultivation of Microalgae .................. 40

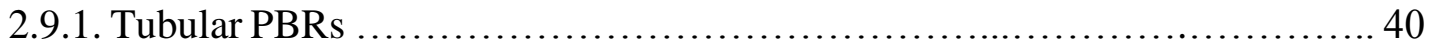


2.9.1.1. Serpentine PBRs ................................................ 41

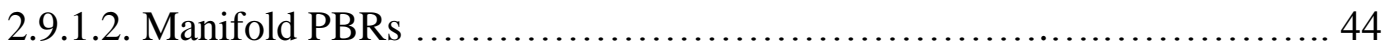

2.9.1.3. Helical PBRs ........................................................ 45

2.9.1.4. Fence Arrangement PBRs with Manifolds ............................. 46

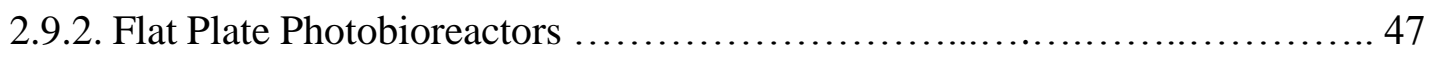

2.9.2.1. Flat Alveolar Panels ............................................... 48

2.9.2.2. Glass plate PBRs ............................................... 49

2.9.2.3. Critical Evaluation of Flat Plate PBRs ............................... 50

2.9.3. Airlift Column PBRs ................................................... 50

2.9.4. Vertical Sleeve PBRs .................................................... 54

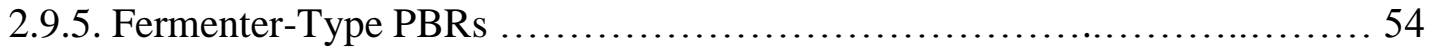

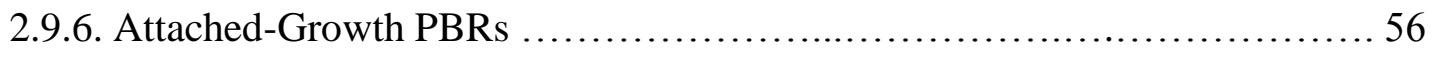

2.10. A Model Photobioreactor Located in Almeria (Spain) ........................... 56

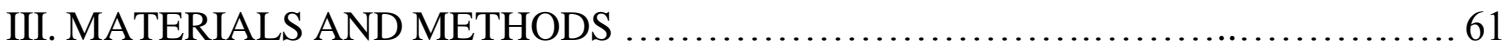

3.1. Prototype Design Criteria ...................................................... 61

3.2. Design Capacity and Geometry .......................................... 63

3.3. Selection of Construction Materials ...........................................68

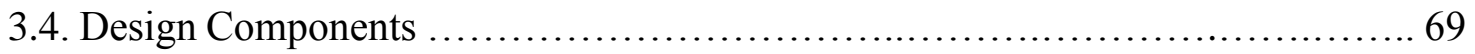

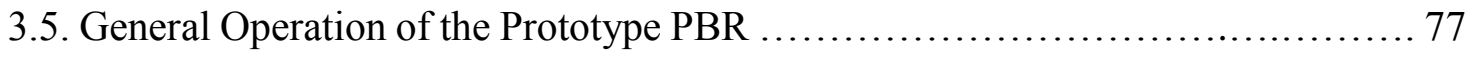

3.6. Method for Fluid Dynamics and Mass Transfer Characterization...................79

3.7. Method for Evaluation of Biomass Productivity of the Prototype PBR .......... 81

3.7.1. Direct Microscopic Counting .......................................... 82

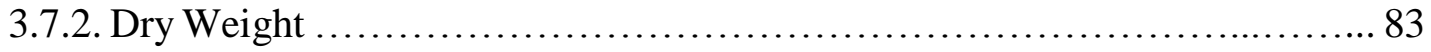


3.8. Methods for Theoretical Scale-Up of the Prototype PBR

3.9. Method for Evaluation of Power Consumption of PBR in Almeria (Spain) .... .85

3.9.1. Evaluating the Specific Power Input of the Air Blower .................. 86

3.9.2. Evaluating the Specific Power Input of the Centrifugal Pump .............. 87

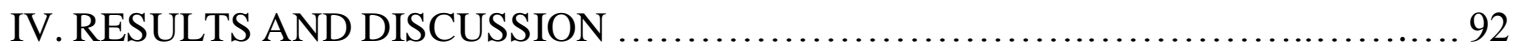

4.1. Fluid Dynamics and Mass Transfer Characterization ........................ 92

4.1.1. Theoretical Scale-Up of the Cal Poly Prototype PBR ..................... 102

4.1.2. Comparison of Prototype PBR to Model System in Almeria (Spain) in Terms of Specific Power Input ................................ 105

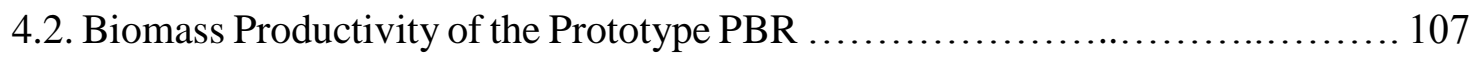

4.2.1. Operation of Batch Run $\# 1$....................................... 107

4.2.2. Operation of Batch Run $\# 2$....................................... 113

4.2.3. Comparison of Prototype PBR to Model System in Almeria (Spain)

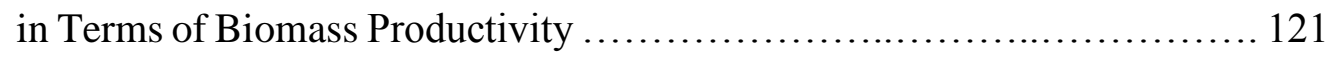

4.3. Biofouling in the Prototype PBR .................................... 127

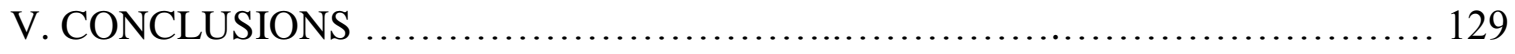

5.1. Experimental Conclusions ......................................... 129

5.2. Future Research ............................................... 131

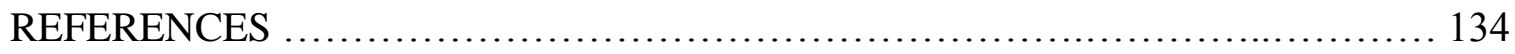

\section{APPENDICES}

A. Total Suspended Solids Data ....................................... 144

B. ${\mathrm{N}-\mathrm{NO}_{3} \text { Concentration Data } \ldots \ldots \ldots \ldots \ldots \ldots \ldots \ldots \ldots \ldots \ldots \ldots \ldots \ldots \ldots \ldots \ldots \ldots \ldots \ldots \ldots \ldots} 146$

C. Dissolved Oxygen Concentration Data ................................ 147 
D. Fluid Dynamics and Mass Transfer Characterization Data ................... 148

E. Calculations for Almeria Centrifugal Pump Power Input ..................... 149

F. Head loss Calculations for Projected 3,000-L Cal Poly PBR .................. 150

G. Dissolved Oxygen Profiles at $0.06 \mathrm{v} / \mathrm{v} / \mathrm{min}$ Aeration Rate $\ldots \ldots \ldots \ldots \ldots \ldots \ldots \ldots \ldots 1$

H. Dissolved Oxygen Profiles at $0.10 \mathrm{v} / \mathrm{v} / \mathrm{min}$ Aeration Rate ................... 152

I. Dissolved Oxygen Profiles at 0.15 v/v/min Aeration Rate ................... 153

J. Dissolved Oxygen Profiles at 0.20 v/v/min Aeration Rate ................... 154

K. Dissolved Oxygen Profiles at $0.25 \mathrm{v} / \mathrm{v} / \mathrm{min}$ Aeration Rate ................... 155

L. Dissolved Oxygen Profiles at 0.30 v/v/min Aeration Rate .................... 156

M. Dissolved Oxygen Profiles at 0.35 v/v/min Aeration Rate .................... 157

N. Dissolved Oxygen Profiles at 0.40 v/v/min Aeration Rate .................... 158

O. Dissolved Oxygen Profiles at 0.45 v/v/min Aeration Rate ................... 159

P. Dissolved Oxygen Profiles at $0.50 \mathrm{v} / \mathrm{v} / \mathrm{min}$ Aeration Rate $\ldots \ldots \ldots \ldots \ldots \ldots \ldots \ldots \ldots$

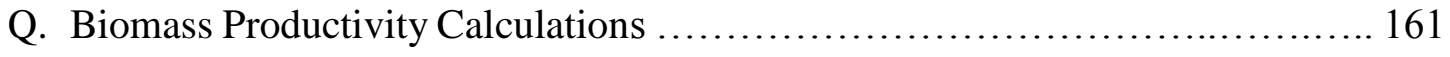

R. Airlift calculations for the 3,000-L Cal Poly PBR ........................... 163 


\section{LIST OF TABLES}

Table

Page

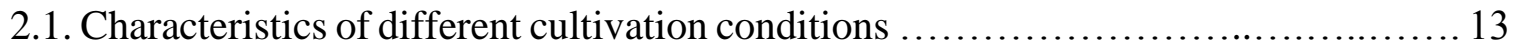

2.2. Advantages and disadvantages of open and closed algal cultivation systems ...........18

2.3. Present state of large-scale microalgal production ............................. 24

2.4. Cultivation systems of start-ups and new small firms in the field .................. 26

2.5. Recording microalgal productivities in PBRs and ponds ............................... 36

2.6. Specifications of the Almeria PBR facility when growing Scenedesmus

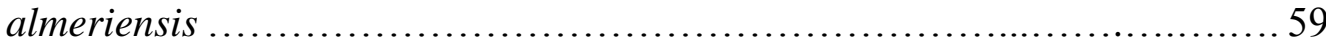

2.7. Power consumption of the main equipment used in the Almeria facility .............. 60

2.8. Power consumption of the air blower and centrifugal pump for a single

3,000-L PBR unit in the Almeria facility ............................... 60

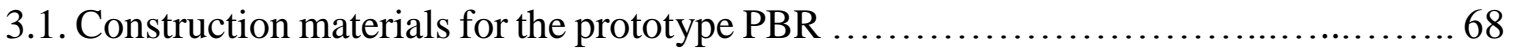

3.2. Components and concentrations for $1 \mathrm{~L}$ of Bristol medium and $10 \mathrm{~mL}$

of stock solution of each component $\ldots \ldots \ldots \ldots \ldots \ldots \ldots \ldots \ldots \ldots \ldots \ldots \ldots \ldots \ldots \ldots$

3.3. Almeria PBR specifications used to calculate the specific power input

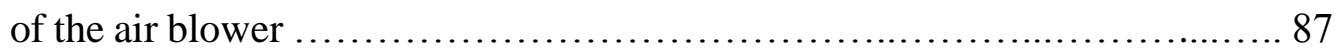

3.4. Almeria PBR specifications used to calculate the specific power input

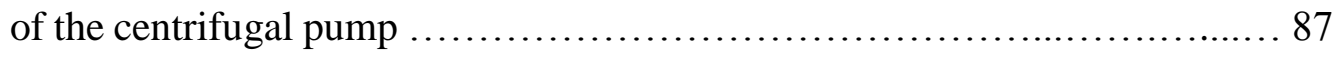

3.5. Assumptions used to calculate the specific power input of the centrifugal

pump of the Almeria PBR ............................................. 88

4.1. Comparison of overall mass transfer coefficients $\left(K_{L} a\right)$ in different

closed microalgal culture systems ................................... 96 
4.2. Specifications to calculate the specific power input of the air blower for the projected 3,000-L Cal Poly PBR

4.3. Assumptions to calculate the specific power input of the air blower for the projected 3,000-L Cal Poly PBR 104

4.4. Turbine blower specifications 105

4.5. Specific power input comparison between PBR units 106

4.6. Specifications for the Almeria and prototype PBRs

5.1. Specific biomass productivities of some PBRs 


\section{LIST OF FIGURES}

Figure

Page

1.1. A schematic view of a horizontal tubular PBR

2.1. Past attempts of large-scale microalgae production 11

2.2. Hybrid systems. $(a, b)$ Different configurations of a C-shaped fiberglass integrated open pond-PBR designed by Mottahedeh \& Tredici (2012); (c) pressurized polyethylene bags at Algenol BioFuels, Inc. (Florida, USA), for bioethanol production; (d) detail of the condensed water-ethanol vapor on the reactor wall (e) and scheme of the process; (f) Algae Tunnel system at AlgaeStream SA (France), the inset shows the interior of a tunnel

2.3. Floating Systems. (a) Floating tubular reactor designed by C. Gudin at Heliosynthesis in the 1980s; (b) floating PBR developed by Algasol Renewables SL (Spain); (c) low-density polyethylene tubular OMEGA (Offshore Membrane Enclosures for Growing Algae) photobioreactor developed by NASA (USA), at San Francisco OMEGA experimental site; (d) 0.4-ha algae floating pond developed at Utah State University Research Foundation and deployed on a wastewater pond at the Bingham Research Center in Utah, USA

2.4. Flow around a turbine blade 32

2.5. A horizontal tubular photobioreactor

2.6. Serpentine systems of the $1990 \mathrm{~s}$

2.8. A 1000-L helical PBR at Murdoch University, Australia 46

2.9. Biofence 
2.10. A flat plate photobioreactor ................................................... 48

2.11. Different types of airlift column PBRs ............................................ 51

2.12. Airlift columns ............................................................... 52

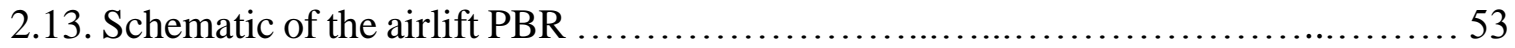

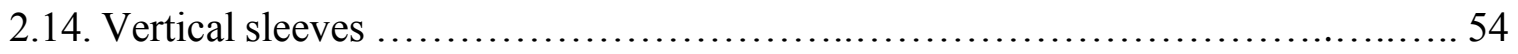

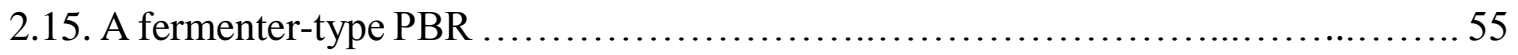

2.16. A 10-unit fence-type configuration totaling 30,000 L located in

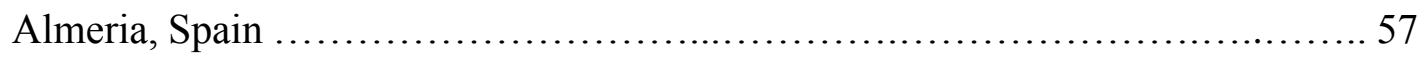

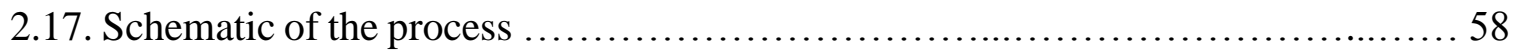

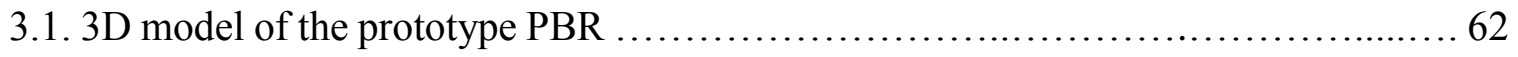

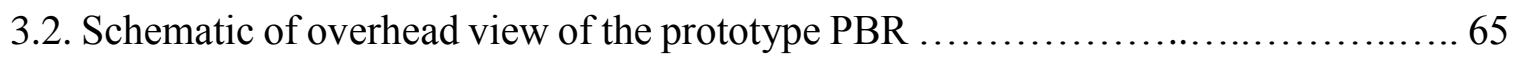

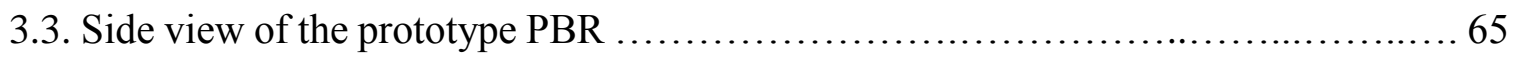

3.4. Schematic of side view of the prototype PBR showing culture exit port of the airlift column; culture flows from left to right (continues on next page).......... 66

3.5. Schematic of side view of the prototype PBR showing culture entrance port of the airlift column; culture flows from left to right ..................... 67

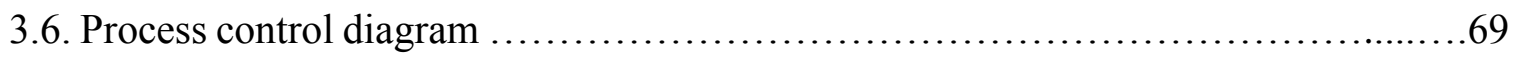

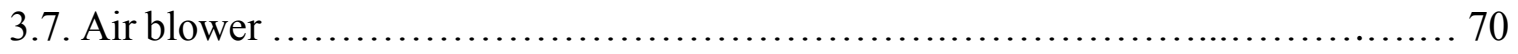

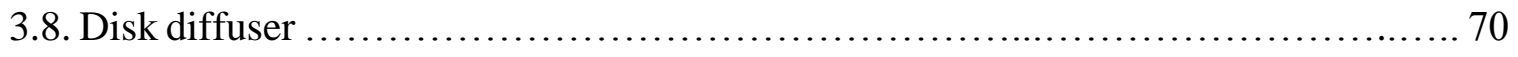

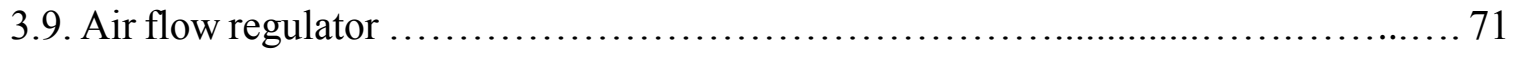

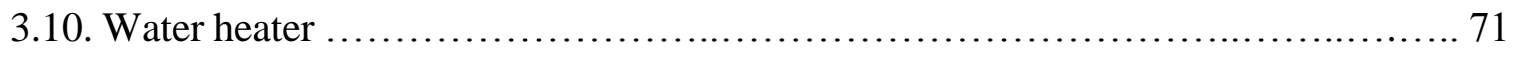

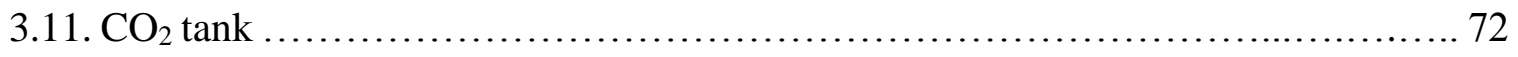

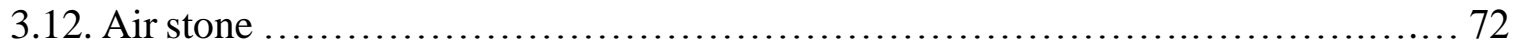




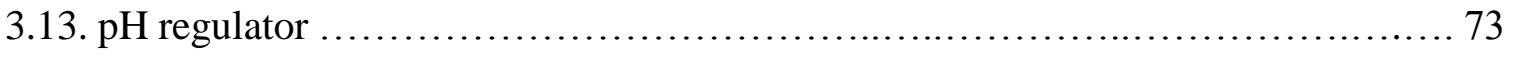

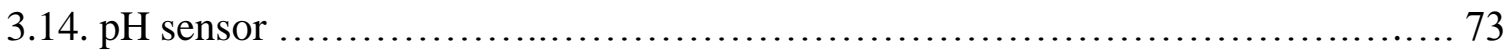

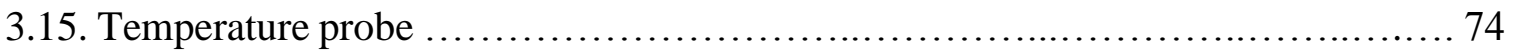

3.16. Nitrate ion-selective electrode ............................................... 74

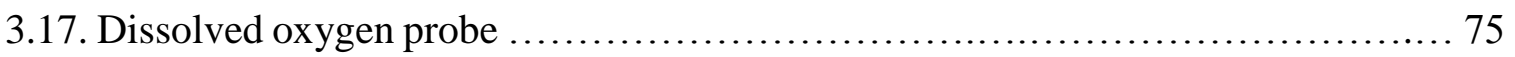

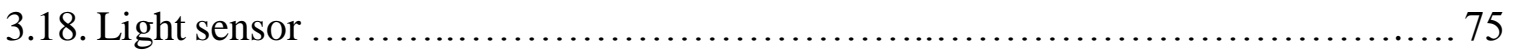

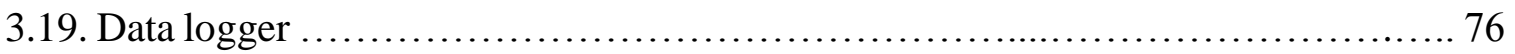

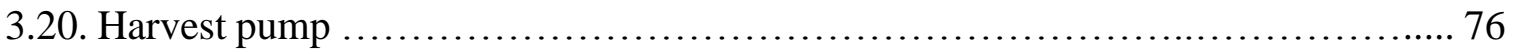

3.21. Culture of $C$. vulgaris in 1-L Bristol medium .................................... 77

3.22. Culture of $C$. vulgaris in a 20-L carboy with Bristol medium ............................ 78

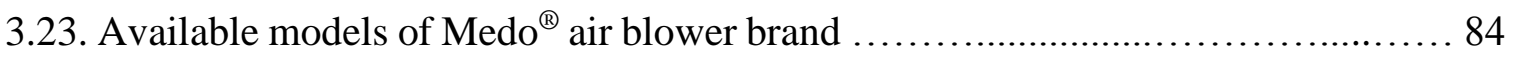

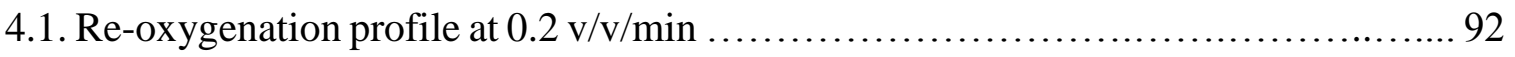

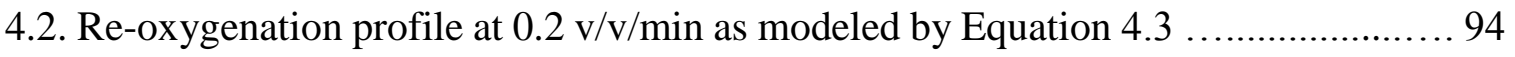

4.3. Influence of the aeration rate on the gas holdup and overall oxygen transfer coefficient of the prototype PBR ...................................... 95

4.4. Influence of the aeration rate on the Reynolds number and mixing time .............. 99

4.5. Influence of the aeration rate on the Reynolds number and overall oxygen transfer coefficient of the PBR .............................................. 100

4.6. Influence of specific power supply on aeration rate to the PBR and

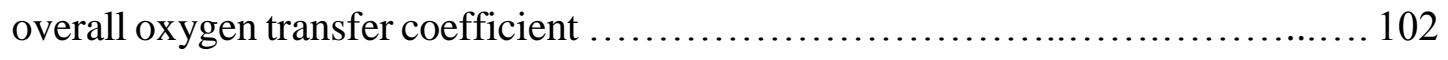

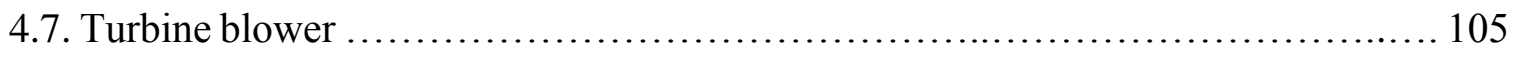

4.8. PBR at time of inoculation .................................................. 108

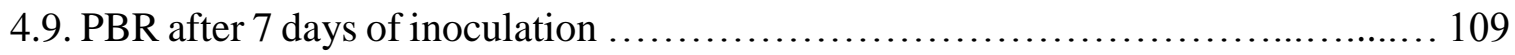


4.10. PBR after 14 days of inoculation

4.11. $\mathrm{N}-\mathrm{NO}_{3}$ and cell concentration data measured over 28 days ....................... 111

4.12. PBR temperature profile measured over 3 days ................................... 112

4.13. Temperature profile of the PBR liquid measured over 19 days ........................... 112

4.14. $\mathrm{pH}$ profile of the PBR measured over 21 days ................................ 113

4.15. Growing the $2^{\text {nd }}$ batch of $C$. vulgaris for 17 days in the PBR ................... 115

4.16. Cell concentration data measured for both batch runs ................................ 116

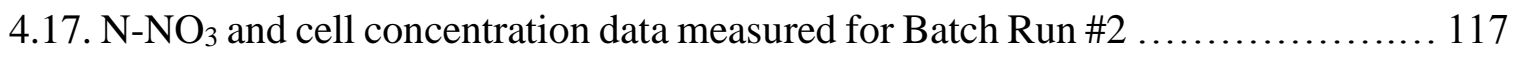

4.18. $\mathrm{N}-\mathrm{NO}_{3}$ concentration and average biomass dry weight measured

for Batch Run \#2 ….......................................... 117

4.19. $\mathrm{pH}$ profile for Batch Run \#2 measured over 17 days ............................. 119

4.20. Temperature profile for Batch Run \#2 measured over 17 days .................... 120

4.21. Illumination profile for Batch Run \#2 measured over 17 days ..................... 120

4.22. Dissolved oxygen profile for Batch Run \#2 as \%Sat .............................. 121

4.23. Biomass dry weight data from exponential growth phase of

Batch Run \#2 as modeled by Equation 4.12 ..................................... 123

4.24. Two side views of the reactor showing biofouling of the PBR tubes and columns after draining the culture 127

4.25. Two side views of the reactor after cleaning the biofouling 128 


\section{CHAPTER I: INTRODUCTION}

The design of energy efficient systems for the growth of microalgae is important due to the enormous potential for algae-based products in numerous applications. Microalgae can produce many bioactive compounds, such as antifungal, antibacterial, antiviral substances, biodiesel and high-value compounds, such as carotenoids and phycobiliproteins for human and animal nutrition (Spolaore et al., 2006). Microalgae are also being studied for $\mathrm{CO}_{2}$ fixation, removal of nitric oxide from flue gas, recovery of heavy metals and life-support systems for spacecraft (Carvalho et al., 2006).

Thousands of species of algae are known to date and only a handful of these have been investigated (Tredici, 2004). Currently a few microalgal species are being cultivated for commercial purposes, but many other species are being studied in detail as potential sources of high-value products (Olaizola, 2003). For example, algae can be used as a source of drugs as an alternative to chemical synthesis (Olaizola, 2003).

State-of-the-art microalgal production systems currently fall under two major classifications: closed systems (photobioreactor technology) and open systems (openpond technology). Photobioreactors (PBRs) can be defined as culturing systems where the light passes through reactor walls (usually made of a transparent, UV-resistant material) to reach all the cultivated cells (Tredici, 2004). Open ponds, on the other hand, are open systems where sunlight impinges on the surface of the culture. (Tredici, 2004).

Both types of cultivation systems have their advantages and drawbacks. Open ponds are simple and relatively inexpensive to build, require minimal labor for operation and maintenance, but are more susceptible than PBRs to microbial and other sources of 
contamination, and suffer from water losses through evaporation (Davis et al., 2011). PBRs lower the risk of contamination and water losses and provide greater control of variables that affect algae growth, but these features come at the expense of limited scalability and high capital, maintenance, and operating costs (Davis et al., 2011).

To date there is no such thing as "the best bioreactor system" that possesses all the advantages of open ponds and PBRs to achieve a relatively maximum biomass productivity at relatively minimum capital and operating costs (Carvalho et al., 2006). In fact, the choice of reactor design is situation dependent, which means that the algal species and the final purpose will play a role (Carvalho et al., 2006). Thus, PBRs and open ponds should not be seen as competing technologies, but more accurately as separate advancements in the field of microalgal biotechnology (Tredici, 2004). Moreover, hybrid systems that combine features of PBR and open-pond technology are being developed, and combined production processes that couple ponds and PBRs are becoming common practice in the algae industry (Zittelli et al., 2013).

Open pond technology is the first-generation technology for commercial production of microalgae and is currently the technology of choice for most commercial applications (Davis et al., 2011). Open ponds have been extensively studied by Dodd (1986), Richmond \& Becker (1986), Oswald (1988), Becker (1994) Chisti (2007), and Zittelli et al. (2013). PBR technology has been reviewed by Lee (1986), Chaumont (1993), Prokop \& Erickson (1995), Torzillo (1997), Tredici \& Chini Zittelli (1997), Pulz \& Scheibenbogen (1998), Tredici (1999), Chisti et al. (1999), Chisti (2007), and Zittelli et al., (2013). Technological development of PBRs is lacking (Zittelli et al., 2013). Although a good number of PBR designs have been proposed, very few are capable of 
sustaining commercial production of microalgae (Zittelli et al., 2013). Thus the need arises for improved PBR designs.

Tubular and airlift-column PBRs are the best known and most common PBR designs to date, but there is room for power consumption improvements on these designs. Airliftcolumn PBRs have been extensively studied since the 1940s (Tredici, 2004), while tubular PBRs have been well known since the 1950s (Tredici, 2004). An airlift-column unit alone can serve as a PBR, but is limited in size to a few meters in height. Traditional tubular PBR designs have greater potential for scaling up and have an airlift-column stage as part of the reactor system (Figure 1.1). Published literature depict the outstanding photosynthetic efficiency of tubular and airlift-column PBRs and show promising results for industrial-scale algae cultivation (Zittelli et al., 2013). A limiting factor associated with tubular PBR designs is power consumption of electrically powered components. Minimizing required power consumption should make this type of PBR more attractive since this could in turn decrease its operating costs significantly.

Tubular PBR designs normally need an air compressor or blower and a centrifugal pump for culture circulation and gas exchange (Carlvalho et al., 2006; Cathcart, 2011; Hulatt \& Thomas, 2011). In a tubular PBR the air flow provides gas exchange by stripping out excess dissolved oxygen in an airlift-column unit, where the $\mathrm{CO}_{2}$ is injected, while the centrifugal pump circulates the culture in the tubular stage and decreases the amount of air bubbles traveling into this stage (Figure 1.1). The PBR design in this thesis research uses an air blower to perform gas exchange in its airlift columns and system-wide circulation without any significant over-accumulation of dissolved oxygen. The design herein builds on previous tubular PBR designs that provide circulation and gas exchange 
by airlift alone (Travesio et al., 2001; Cathcart, 2011). However, Travesio et al. (2001) and Cathcart (2011) do not provide data on gas transfer, energy consumption, flow regime or biomass productivity. The PBR design herein does away with the centrifugal pump for a tubular stage of a PBR for significant reduction in electrical power consumption.

Pegallapati et al. (2014) reported that microalgae cultivation systems have normally been evaluated and optimized for volumetric biomass productivity $\left(\mathrm{g} \mathrm{L}^{-1} \mathrm{~d}^{-1}\right)$ without considering energy input. An assessment performed by Pegallapati et al. (2014) with data compiled on a variety of PBRs showed that they don't normally perform well in terms of energy efficiency on a basis of energy input to the cultivation process (i.e., g Joule ${ }^{-1}$ or $g$ $\mathrm{W}^{-1} \mathrm{~d}^{-1}$ ) and this parameter has been overlooked. Energy spent during cultivation must be minimized to minimize production costs (Pegallapati et al., 2014).

The objective of this research was to investigate a new photobioreactor (PBR) design for microalgae production that retains the typical advantages of existing tubular PBRs while reducing power consumption by providing simultaneous culture circulation and gas exchange with airlift alone and no centrifugal recirculating pump. The specific objectives included:

1. Design and build a prototype PBR with a configuration that has the advantages of a tubular-type PBR, relatively high volume-to-land-area ratio, and can perform circulation and gas exchange with airlift alone 
2. Perform a fluid dynamics and mass transfer characterization of this design by determining its gas holdup capacity, oxygen transfer capacity, specific power requirements and mixing capacity with respect to aeration rate.

3. Operate the PBR and evaluate its productivity by determining its volumetric productivity (g biomass $\mathrm{L}^{-1}$ reactor working volume day ${ }^{-1}$ ), illuminated surface productivity ( $\mathrm{g}$ biomass $\mathrm{m}^{-2}$ reactor photo-stage day ${ }^{-1}$ ), overall areal productivity ( $\mathrm{g}$ biomass $\mathrm{m}^{-2}$ total land area with empty space day ${ }^{-1}$ ) and specific biomass productivity (mg biomass $\mathrm{kJ}^{-1}$ energy needed for culture circulation and gas exchange) with Chlorella vulgaris as the model algae.

4. Perform a power consumption and biomass productivity analysis to assess the economic feasibility of operation of this prototype PBR as compared to similar PBR designs currently used in industry.

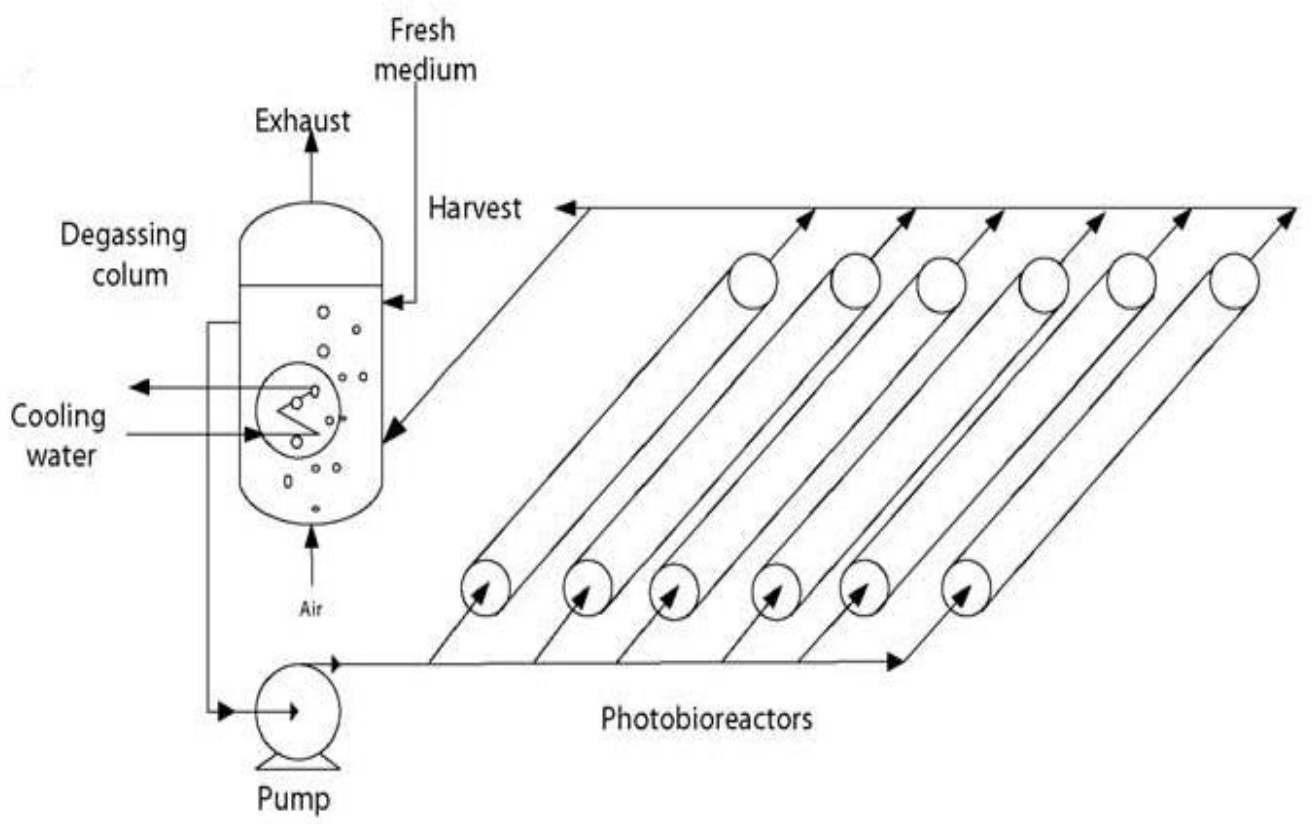

Figure 1.1. A schematic view of a horizontal tubular PBR (Jorquera et al., 2010) 


\section{CHAPTER II: BACKGROUND}

\subsection{Concepts of Photobioreactor (PBR) Engineering}

As mentioned, there are currently two major classifications of microalgal production systems: closed systems (photobioreactor technology) and open systems (open-pond technology). Photobioreactors (Figure 1.1) can be defined as culturing systems where the light passes through reactor walls (usually made of a transparent, UV-resistant material) to reach all the cultivated cells (Tredici, 2004). Open ponds, on the other hand, are open systems where most of the light impinges directly on the surface of the culture (Tredici, 2004).

Natural and man-made open pond algae production is simple technology, but some contend that is not economical on the basis of unit of biomass produced (Oilgae, 2013). Relatively low biomass productivity, contamination and water losses are the main drawbacks of open pond cultivation systems (Oilgae, 2013). The need to overcome these issues has created incentives to build and develop photobioreactors (Oilgae, 2013).

A commercial scale microalgae production plant based on PBRs must have as many of the following characteristics as possible to lower the total cost of biomass harvested (Olaizola, 2003):

- High areal productivity $\left(\mathrm{g} \mathrm{m}^{-2} \mathrm{day}^{-1}\right)$, because some operation costs are proportional to plant size

- High volumetric productivity $\left(\mathrm{g} \mathrm{L}^{-1} \mathrm{day}^{-1}\right)$, because some operation costs are proportional to the amount of water needed for culturing 
- Large volume per PBR unit $\left(\mathrm{L} \mathrm{PBR}^{-1}\right)$, because some operation costs scale with the number of PBR modules needed

- Inexpensive to build and maintain $\left(\$ \mathrm{~m}^{-3}\right)$

- Easy to control culture parameters (temperature, $\mathrm{pH}, \mathrm{O}_{2}, \mathrm{CO}_{2}$, mixing)

- Reliability of operation

- Energy efficiency in terms of biomass productivity per energy input

Note: $\mathrm{L}=$ liter, $\mathrm{g}=$ gram, $\mathrm{m}=$ meter, $\$=$ expense.

Different PBR designs have attempted to achieve these characteristics in different ways, but the optimum PBR design will be the one that can produce high value products with the best possible quality for the least amount of money (Olaizola, 2003).

\subsection{A Brief History of Microalgae Cultivation Technology Development}

Human consumption of naturally occurring microalgae has occurred for centuries (Jensen, 2002). The Chinese population consumed Nostoc to survive famine about 2000 years ago while indigenous populations around the globe have used Spirulina and Aphanizomenon as a source of food for thousands of years (Jensen, 2002). Microalgae cultivation, or farming, has been practiced for only the last few decades and the biotechnology to cultivate microalgae only began to develop in the middle of the last century (Spolaore, 2006).

\subsubsection{Technological Developments from the Early 1950s to the 1990s}

According to Spolaore (2006), in the early 1950s with world population increasing exponentially and fears of a shortage of food supply, scientists began to look for unconventional sources of protein for human nutrition. At the time algal biomass appeared 
to be a good candidate to satisfy these needs, and with a systematic study of microalgae in the pursuit of useful bioactive molecules, the search for algae-based antibiotics began.

The use of microalgae as a source of renewable energy instilled interest in experts confronting the energy crisis of the 1970s (Spolaore, 2006). Interest in the potential of algae continued with studies of algae as photosynthetic reactors and gas exchangers for space travel as a way to convert human $\mathrm{CO}_{2}$ released into breathable oxygen (Spolaore, 2006). In the late 1960s and 1970s, Advanced Integrated Wastewater Pond Systems (AIWPSs) that use microalgae to enhance the quality of wastewater began to be built in the United States (Green et al., 1995). The basis of this technology is the use of microalgae as a source of $\mathrm{O}_{2}$ for aerobic digestion of sewage and fermentation of the resulting algal biomass from $\mathrm{CO}_{2}$ absorption to produce methane as fuel to power water treatment facilities (Green et al., 1995).

Commercial-scale algae production began in the 1960s in Japan by Nihon Chlorella Co. Ltd. growing Chlorella, and this was followed by the establishment of Sosa Texoco S.A. in Mexico to grow and harvest Arthrospira (Spolaore, 2006). And in the 1970s the first aquaculture fields, for growing aquatic organisms under controlled conditions, began to appear (Spolaore, 2006).

By 1980, there were 46 large-scale plants in Asia producing more than $1000 \mathrm{~kg}$ of microalgae per month; most of which were Chlorella (Spolaore, 2006). In 1986, the establishment of Western Biotechnology Ltd. and Betatene Ltd. production facilities in Australia to grow Dunaliella salina and isolate $\beta$-carotene became a reality (Spolaore, 2006). These were soon followed by construction of commercial plants in Israel, the USA 
and India at around the same time for large-scale production of blue-green algae, such as Spirulina (Spolaore, 2006).

Of special interest in the early 1990s was unconventional production of biodiesel from microalgae as a transportation fuel (Chisti, 2007). Research in the early 1990s by the National Renewable Energy Laboratory (NREL) showed that under controlled conditions algae are capable of producing 40 times the amount of oil for biodiesel per unit area of land, compared to terrestrial oilseed crops such as soy and canola (Sheehan et al., 1998).

\subsubsection{Attempts of Large-Scale PBR Microalgae Facilities in the 1990s}

Several large-scale algae PBR production systems were built in the 1990s, although most of these production plants closed after a few months of operation (Tredici, 2004). The following is a brief description of a few of these failed commercial plants. These descriptions depict that PBR technology needed a great deal of improvement in the 1990s.

\subsubsection{Sagdiana PGT (Tadshikistan)}

A large-scale horizontal manifold PBR was built in 1991 in Javan (Tadshikistan), by Sagdiana PGT, which consisted of ten separate units for a total of $41,000 \mathrm{~m}$ of tubing (Figure 2.1.a). The tubes were $5.7 \mathrm{~cm}$-ID-glass tubes, each 73.5-m long (Tredici, 2004). However, the plant was operated for just three months (Tredici, 2004). The main

problems reported were low volumetric productivity $\left(<0.1 \mathrm{~g} \mathrm{~L}^{-1} \mathrm{~d}^{-1}\right)$, instability of the production process and contamination by Scenedesmus (Tredici, 2004). 


\subsubsection{Photo Bioreactors Ltd (Spain)}

Photo Bioreactors Ltd, established in Spain in the late 1980s, was one of the biggest disasters in the field of microalgal biotechnology. The company attempted two different reactor designs patented by John Pirt (1983). The research and design done by Mr. Pirt at Queen Elizabeth College, which indicated high projected areal productivities (more than $50 \mathrm{~g} \mathrm{~m}^{-2} \mathrm{~d}^{-1}$ ), attracted private and public investors which led to the creation of Photo Bioreactors Ltd in 1986 (Tredici, 2004). After spending three years in the construction of a commercial plant in Murcia (Spain), production of Dunaliella began in May 1990 with a system comprised of 1.2-cm ID, 50-m long polyethylene tubes connected to vertical manifolds making up a total of $125,000 \mathrm{~m}$ of tubing (Figure 2.1.b). Gas exchange in this system was controlled by an airlift column, liquid flow was controlled by centrifugal pumps and the temperature was controlled by shading the reactors with nets or by water spraying (Tredici, 2004). Soon after, another reactor system was built in the same location consisting of a near-horizontal manifold system with 200,000 m of tubing; per the patented Pirt design (1983).

Several operational flaws were reported affecting both systems soon after starting operation. Overly small tube diameter; resulting in a very high S/V ratio in relation to the length of the tubes and poor mixing and circulation, rapid material degradation under sunlight, unavoidable tube wall growth, inadequate degassing and insufficient temperature control were among the most apparent errors (Tredici, 2004). These issues led to poor growth of the algae, biofouling and heavy contamination, resulting in permanent shutdown of the plant in September 1991 (Tredici, 2004). 

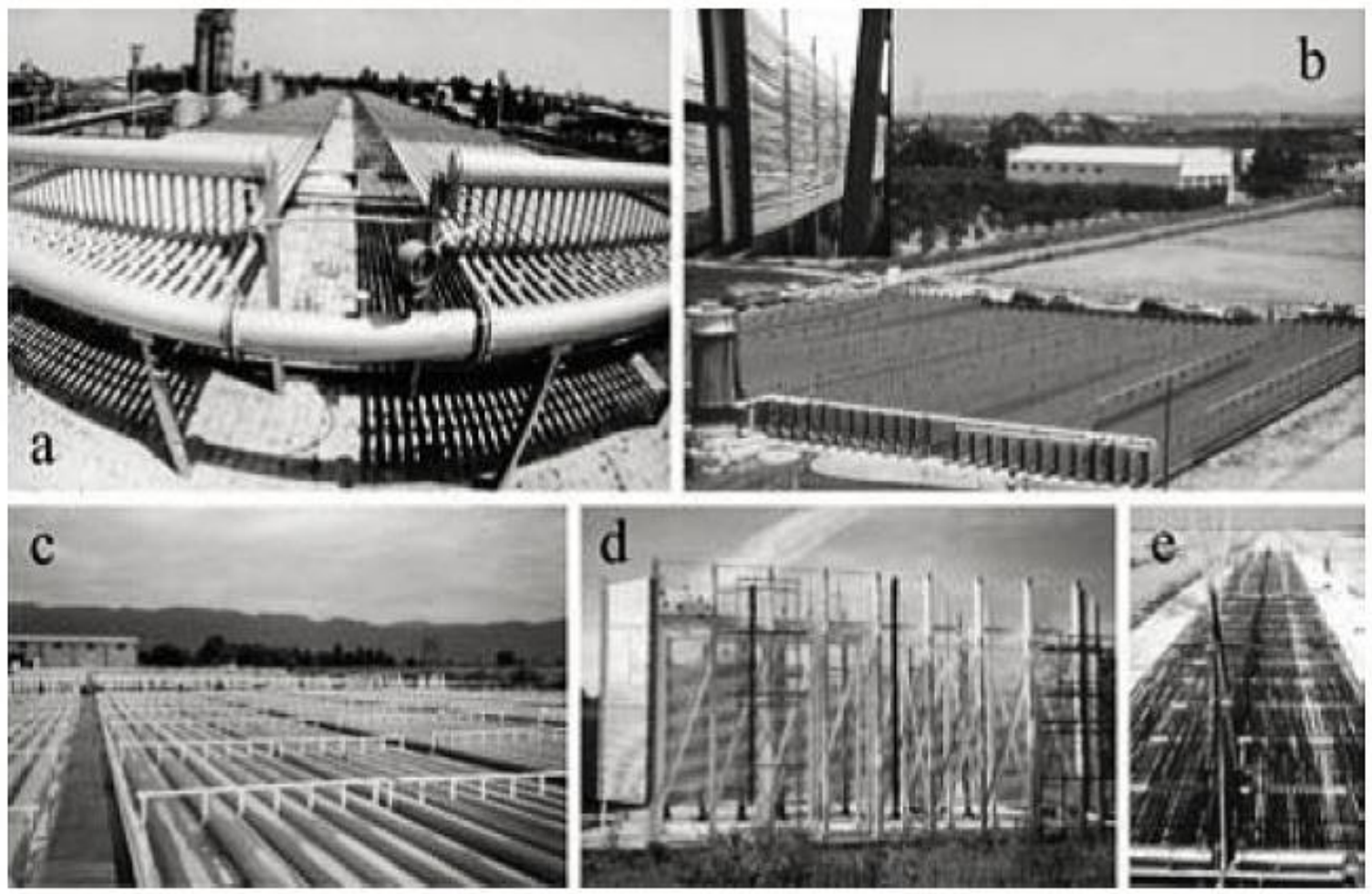

Figure 2.1. Past attempts of large-scale microalgae production (Tredici, 2004)

\subsubsection{Hidrobiologica SA (Argentina)}

According to Tredici (2004), in 1996 Hidrobiologica S.A. built the largest PBR known at the time, about $15 \mathrm{~km}$ south of La Roja (northern Argentina) with an initial investment of $€ 2$ million; which consisted of 96 polyethylene 120-m long and 25.5-cm ID tubes laid parallel to the ground and arranged like a manifold (Figure 2.1.c). The surface of the whole plant was about $5,000 \mathrm{~m}^{2}$ (which included the gaps between modules) and the total culture volume was $600 \mathrm{~m}^{3}$, driven by a single axial flow pump. During the initial period of operation, a volumetric productivity of $0.2 \mathrm{~g} \mathrm{~L}^{-1} \mathrm{~d}^{-1}$ was attained and the biomass produced was of excellent quality. However, several problems began to appear in the long run. The main problems that became apparent were inadequate mixing, biofouling, unequal distribution of culture among the tubes, limited capacity to control the 
temperature, and photosynthetic-oxygen build up to dangerous levels despite a welldesigned degassing system. Thinking that relatively large ID tubing and low flow rate were the causes behind all these problems, the plant went through a complete restructuring to a smaller ID tubing to improve culture circulation. In spite of the changes the plant was closed down in the summer of 1999.

\subsubsection{Inalco S.p.A. (Italy)}

A 3,000-L coiled PBR was built in 1996 by Inalco S.p.A. near Florence (Italy). The unit comprised of 5-cm polycarbonate tubes, 7-m high, connected by elbows and secured to an upright iron hexagonal frame with and inclination to the horizontal of about $1.4^{\circ}$ (Figure 2.1.d). Despite the fact that the reactor successfully grew A. platensis for more than six months, the company closed in 1998 (Tredici, 2004).

\subsubsection{Microalgae S.p.A. (Italy)}

In 1997 Microalgae S.p.A. built a 14-m³ horizontal manifold PBR in Crispiano (Italy) with gas exchange controlled by an airlift column (Tredici, 2004). And in 1999 the company finished expanding the plant to six units comprising of $80-\mathrm{km}$ rigid plastic tubes of about 3-cm OD for a total capacity of $85 \mathrm{~m}^{3}$ (Figure 2.1.e). However, the plant stopped operations in the winter of the same year for unknown reasons (Tredici, 2004).

As depicted in these stories of failed commercial plants, poor circulation and gas exchange have been some of the major obstacles to overcome in order to achieve viable commercial biomass production. Thus, this engineering aspect of PBR design has been overlooked many times and needs attention. 


\subsection{Cultivation Conditions for Microalgae}

Chemical composition and growth characteristics of microalgae are known to depend on cultivation conditions (Chang et al., 2011). Cultivation conditions for microalgae fall into four general categories: photoautotrophic, heterotrophic, mixotrophic and photoheterotrophic cultivation (Chang et al., 2011). Table 2.1 summarizes the characteristics of each cultivation condition, discussed at length in this section.

Table 2.1. Characteristics of different cultivation conditions (Chang et al., 2011)

\begin{tabular}{llllll}
\hline $\begin{array}{l}\text { Cultivation } \\
\text { condition }\end{array}$ & $\begin{array}{l}\text { Energy } \\
\text { source }\end{array}$ & $\begin{array}{l}\text { Carbon } \\
\text { source }\end{array}$ & $\begin{array}{l}\text { Reactor } \\
\text { scale-up }\end{array}$ & Cost & $\begin{array}{l}\text { Issues associated with } \\
\text { scale-up }\end{array}$ \\
\hline Phototrophic & Light & Inorganic & $\begin{array}{l}\text { Open pond or } \\
\text { PBR }\end{array}$ & Low & $\begin{array}{l}\text { Low cell density, } \\
\text { High condensation cost }\end{array}$ \\
Heterotrophic & Organic & Organic & $\begin{array}{l}\text { Conventional } \\
\text { fermentor }\end{array}$ & Medium & $\begin{array}{l}\text { Contamination, } \\
\text { High substrate cost } \\
\text { Mixotrophic }\end{array}$ \\
$\begin{array}{l}\text { Light } \\
\text { and } \\
\text { organic }\end{array}$ & $\begin{array}{l}\text { Inorganic } \\
\text { and } \\
\text { organic } \\
\text { PBR }\end{array}$ & High & $\begin{array}{l}\text { Contamination, } \\
\text { High equipment cost, } \\
\text { High substrate cost } \\
\text { Contamination, } \\
\text { High equipment cost, } \\
\text { High substrate cost }\end{array}$ \\
\hline
\end{tabular}

\subsubsection{Phototrophic Cultivation}

Phototrophic (or autotrophic) cultivation occurs when microalgae utilize light (such as sunlight) as the energy source and inorganic carbon (such as $\mathrm{CO}_{2}$ ) as the carbon source to form chemical energy through photosynthesis (Huang et al., 2010). This type of cultivation condition is the most widely used in industry for scale-up of outdoor systems since there is less risk of contamination from foreign pathogens, and is the method of choice for this research project (Chang et al., 2011). 


\subsubsection{Heterotrophic Cultivation}

Some microalgae species are able to grow under either phototrophic conditions or heterotrophic conditions (Chojnacka \& Marquez-Rocha, 2004). Heterotrophic cultivation occurs when organic carbon is fed to the microalgae under dark conditions (Chojnacka \& Marquez-Rocha, 2004). This cultivation condition solves the issue of limited light energy when growing microalgae outdoors, which in turn hinders high cell density in large-scale outdoor cultures (Chang et al., 2011). Microalgae can assimilate a variety of organic carbon sources (such as glucose, acetate, glycerol, fructose, sucrose, lactose, galactose, and mannose) to grow (Chang et al., 2011). An inherent drawback to heterotrophic cultivation is a high susceptibility to contamination (Chang et al., 2011).

\subsubsection{Mixotrophic Cultivation}

Mixotrophic cultivation occurs when microalgae utilize organic carbon as the energy source and inorganic carbon as carbon source (Chang et al., 2011). This means that the microalgae utilizes organic carbon to evolve $\mathrm{CO}_{2}$ during a respiration process and trap the evolved $\mathrm{CO}_{2}$ from respiration to use it via photosynthesis (Mata et al., 2010). This explains how some species of microalgae can thrive under either phototrophic or heterotrophic conditions, as stated earlier (Chang et al., 2011). Mixotrophic cultivation is rarely used commercially (Chang et al., 2011).

\subsubsection{Photoheterotrophic Cultivation}

Photoheterotrophic cultivation occurs when microalgae uses light as the energy source and organic carbon as the carbon source (Chang et al., 2011). This means that the cells need sugars and light at the same time to grow (Chojnacka \& Marquez-Rocha, 2004). 
Although this type of cultivation is useful for enhancing the production of some highvalue metabolites, the use of this cultivation condition is rarely used due to its high cost and high risk of contamination (Chang et al., 2011).

\subsubsection{Comparison of Different Cultivation Conditions}

Published literature shows that to grow algae for oil production heterotrophic cultivation is the method of choice because it gives better oil productivity than competing cultivation conditions (Chang et al., 2011). But use of sugars as a carbon source in an algae cultivation system not only creates high susceptibility to contamination (Chang et al., 2011). Sugar from food sources, such as soybean, rapeseed, palm, and corn to grow algae diverts from the food supply and the increasing competition for these sources causes these foods, and resulting biofuel, to become increasingly expensive (Campbell, 2008). Moreover, converting rainforests, peatlands, savannas, or grasslands to produce foodcrop based biofuels creates a "biofuel carbon debt" by releasing 17 to 420 times more $\mathrm{CO}_{2}$ than the annual greenhouse gas (GHG) reductions that these biofuels would provide by displacing fossil fuels (Fargione et al., 2008). Commercialization of algae behooves taking into account the cost of carbon raw material input, inherent risks of contamination, as well as scalability. The phototrophic cultivation condition best conforms to all three requirements (Chang et al., 2011).

\subsection{Cultivation of Algae in Open Ponds}

Open ponds can be either natural or artificial, i.e. man-made (Oilgae, 2013). Variations include lakes, lagoons, circular ponds and raceway ponds (Oilgae, 2013). 


\subsubsection{Lakes and Natural Ponds}

Microalgae will grow abundantly if they find a body of water with sufficient nutrients and suitable climatic conditions. If that body of water has specific chemical characteristics, such as $\mathrm{pH}$ and salinity, and relatively stable temperature, the body of water becomes more selective to certain types of algae, and in the extreme, it becomes a monoculture (Tredici, 2004). Natural ponds are defined as cultivation systems with no mixing and minimal control but still requiring a certain amount of work for construction and maintenance, for example, Dunaiella salina ponds (Tredici, 2004).

\subsubsection{Circular Ponds}

Circular open pond design is not very attractive for commercial applications since it requires expensive concrete for construction, high energy input to fulfill mixing requirements, mechanical problems of a long rotating arm and pond-size limitation to about 10,000 $\mathrm{m}^{2}$ (Zittelli et al., 2013). Nevertheless, this design is widely used in Taiwan, Japan and Indonesia for production of Chlorella (Zittelli et al., 2013).

\subsubsection{Raceway Ponds}

In raceway ponds, the algae, water and nutrients circulate around a track (Andersen, 2005). Paddlewheels provide the flow of water and keep the algae suspended in the water (Andersen, 2005). With the paddles the algae can be circulated back to the surface on a regular basis (Andersen, 2005). Also, these ponds are built shallow so the algae can be exposed to sunlight because sunlight penetrates to limited depths (Andersen, 2005). 
The raceway pond is the pond design of choice for most commercial Anthrospira farms (Zittelli et al., 2013). Earthrise Nutritionals LLC (California, USA) and Hainan DIC Microalgae (China) each produce more than 700 tons annually of Anthrospira in about 185 hectares (Zittelli et al., 2013). Cyanotech Co. (Hawaii, USA) and Perry Agro Industries Ltd (India) produce about 300 tons/year and 180 tons/year in about 36 and 52 ha plants, respectively, of Haematococcus pluvialis to obtain the pigment astaxanthin (Zittelli et al., 2013). Smaller facilities are located in Australia, Thailand, France, and California (USA). Note that the status of such facilities is subject to change.

\subsection{Comparison of PBRs to Open Ponds}

Currently there are two major classifications of microalgal production systems: closed systems (photobioreactor technology) and open systems (open-pond technology). The choice of reactor design will depend on the species of alga being grown and the final purpose (Carvalho et al., 2006).

\subsubsection{Pros and Cons of PBRs and Open Ponds}

Table 2.2 depicts major advantages and disadvantages of open and closed algal cultivation systems. The major drawbacks of open systems are high harvesting cost, relatively low areal productivity in terms of land, high evaporative losses and contamination risk. Closed systems are designed to overcome the disadvantages of open systems, but this comes at the expense of high capital and operating costs, and limited scalability; making their commercial scale-up often not economically feasible. Although productivity is not always higher than in ponds and evaporative cooling is used in PBRs, 
so PBR design and climate influence evaporative water losses and productivity in PBRs

(Mehlitz, 2009).

\section{Table 2.2. Advantages and disadvantages of open and closed algal cultivation}

systems (Pulz, 2001; Mehlitz, 2009; Davis et al., 2011; Lundquist pers. com., 2014)

\begin{tabular}{|c|c|c|}
\hline Parameter & Open Systems (Open Ponds) & Closed Systems (PBRs) \\
\hline Contamination risk & Extremely high & Low \\
\hline Space required & High & Low \\
\hline Water losses & Depending on climate & $\begin{array}{l}\text { Almost none if cooling is } \\
\text { provided by means other than } \\
\text { evaporative cooling }\end{array}$ \\
\hline $\mathrm{CO}_{2}$ losses & $\begin{array}{l}\text { High, } \mathrm{CO}_{2} \text {, like } \mathrm{O}_{2} \text {, is lost } \\
\text { through outgassing }\end{array}$ & $\begin{array}{l}\text { Low for tubular PBRs only, } \\
\text { because } \mathrm{CO}_{2} \text { is typically injected } \\
\text { at the beginning of the tubular } \\
\text { solar receiver (photo-stage) }\end{array}$ \\
\hline Number of cultivable species & $\begin{array}{l}\text { Low, monoculture are restricted } \\
\text { to a few algal varieties }\end{array}$ & High \\
\hline Flexibility of production & $\begin{array}{l}\text { Change of production between } \\
\text { the possible varieties is easily } \\
\text { accomplished by cleaning the } \\
\text { pond and restarting with new } \\
\text { alga }\end{array}$ & $\begin{array}{l}\text { Significant biofouling in the } \\
\text { tubes of a photo-stage can make } \\
\text { PBR cleaning more costly }\end{array}$ \\
\hline $\begin{array}{l}\text { Reproducibility of production } \\
\text { parameters such as } \mathrm{pH}, \\
\text { temperature and DO conc. }\end{array}$ & $\begin{array}{l}\text { Not given, dependent on } \\
\text { environmental conditions }\end{array}$ & $\begin{array}{l}\text { Possible within certain } \\
\text { tolerances }\end{array}$ \\
\hline Degree of process control & Low & High \\
\hline Changes in weather & $\begin{array}{l}\text { Makes process control more } \\
\text { difficult, rain and evaporation } \\
\text { significantly affect areal } \\
\text { productivity }\end{array}$ & $\begin{array}{l}\text { Clouds decrease insolation and } \\
\text { weather affects cooling }\end{array}$ \\
\hline $\begin{array}{l}\text { Period until maximum biomass } \\
\text { concentration is reached after } \\
\text { start }\end{array}$ & Depends on climate and strain & Depends on climate and strain \\
\hline Maximum biomass concentration & Low, approx. 0.1-0.2 $\mathrm{g} \mathrm{L}^{-1}$ & High, approx. 2-8 g L ${ }^{-1}$ \\
\hline Capital investment & Low & High \\
\hline Ease of scale-up & Good & Difficult \\
\hline Availability of technology & Readily available & $\begin{array}{l}\text { Not demonstrated economically } \\
\text { on large scale }\end{array}$ \\
\hline Downstream processing cost & High (very dilute culture) & Low (higher density culture) \\
\hline Area-to-volume ratio & $\begin{array}{l}\text { Large ( } 4-10 \text { times higher than } \\
\text { closed counterpart) }\end{array}$ & small \\
\hline $\begin{array}{l}\text { Main criteria for species } \\
\text { selection }\end{array}$ & Growth competition & $\begin{array}{l}\text { Resistance to hydrodynamic } \\
\text { shear }\end{array}$ \\
\hline Harvesting effort & Low & High \\
\hline Light utilization efficiency & Poor/fair & Fair/excellent \\
\hline Most costly parameters & Mixing & $\begin{array}{l}\text { Oxygen control, temperature } \\
\text { control }\end{array}$ \\
\hline $\begin{array}{l}\text { Main limitation for biomass } \\
\text { productivity }\end{array}$ & Surface area & Volume \\
\hline
\end{tabular}




\subsubsection{Coupling Ponds and Photobioreactors}

The main drawback of raceway ponds is their susceptibility to contamination, while for PBRs it is the high capital and operating costs. A combination of these systems seems promising for cost-effective cultivation of selected algal strains (Zittelli et al., 2013). Adoption of this combined scheme for a two-stage cultivation process has been studied by Rodolfi et al. (2008). In this process, the first stage consists of producing the inoculum in the PBR and the second stage is mass production of the strain to obtain the main product (Zittelli et al., 2013).

Integrated systems are quickly gaining ground in the algae industry. Huntley \& Redalje (2006) describe the use of a 25,000-L tubular PBR coupled with a 50,000-L open pond for both oil and astaxanthin production from $H$. pluvialis, with an annual biomass productivity of $38 \mathrm{t} \mathrm{ha}^{-1}$. The former HR BioPetroleum, Inc. (Hawaii, USA) developed and patented a dual cultivation process, which uses PBRs for continuous cultivation and open ponds for batch cultivation. This system was used to investigate $\mathrm{CO}_{2}$ mitigation coupled with biofuel production (Zittelli et al., 2013). The same company also built a 2.5ha demonstration facility in Hawaii in 2011 to grow marine algae for production of valuable products used in aquaculture and animal feeds (Zittelli et al., 2013).

Hybrid systems combine features of open ponds and PBRs. Two main types of hybrid systems can be found (Zittelli et al., 2013). One is represented be a covered open pond; the concept reduces the possibility of contamination, evaporative losses, and $\mathrm{CO}_{2}$ desorption (Zittelli et al., 2013). The other type is a partially filled tubular design widened and inflated to approximate an open pond; this design is mainly aimed at reducing costs (Zittelli et al., 2013). 
Hybrid systems have been used commercially for over a decade. Genifuel Corp. (Utah, USA) designed a covered raceway pond to grow algae for gasification (Zittelli et al., 2013). Mottahedeh \& Tredici (2012) proposed a bubble-mixed hybrid system that consists of a C-shaped open top bioreactor chamber made of fiberglass sheet and an external removable cover (also made of fiberglass) for creating a closed chamber. XL Renewables, Inc., Phyco Biosciences, Inc. (Arizona, USA), Licamele \& White (2011), MBD Energy Ltd (Melbourne, Australia), Algenol Biofuels, Inc. (Florida, USA), and AlgaeStream SA (Paris, France) have developed and patented similar designs (Figure 2.2). These designs nevertheless may be improved in terms of oxygen removal, thermoregulation and biofouling (Zittelli et al., 2013). 

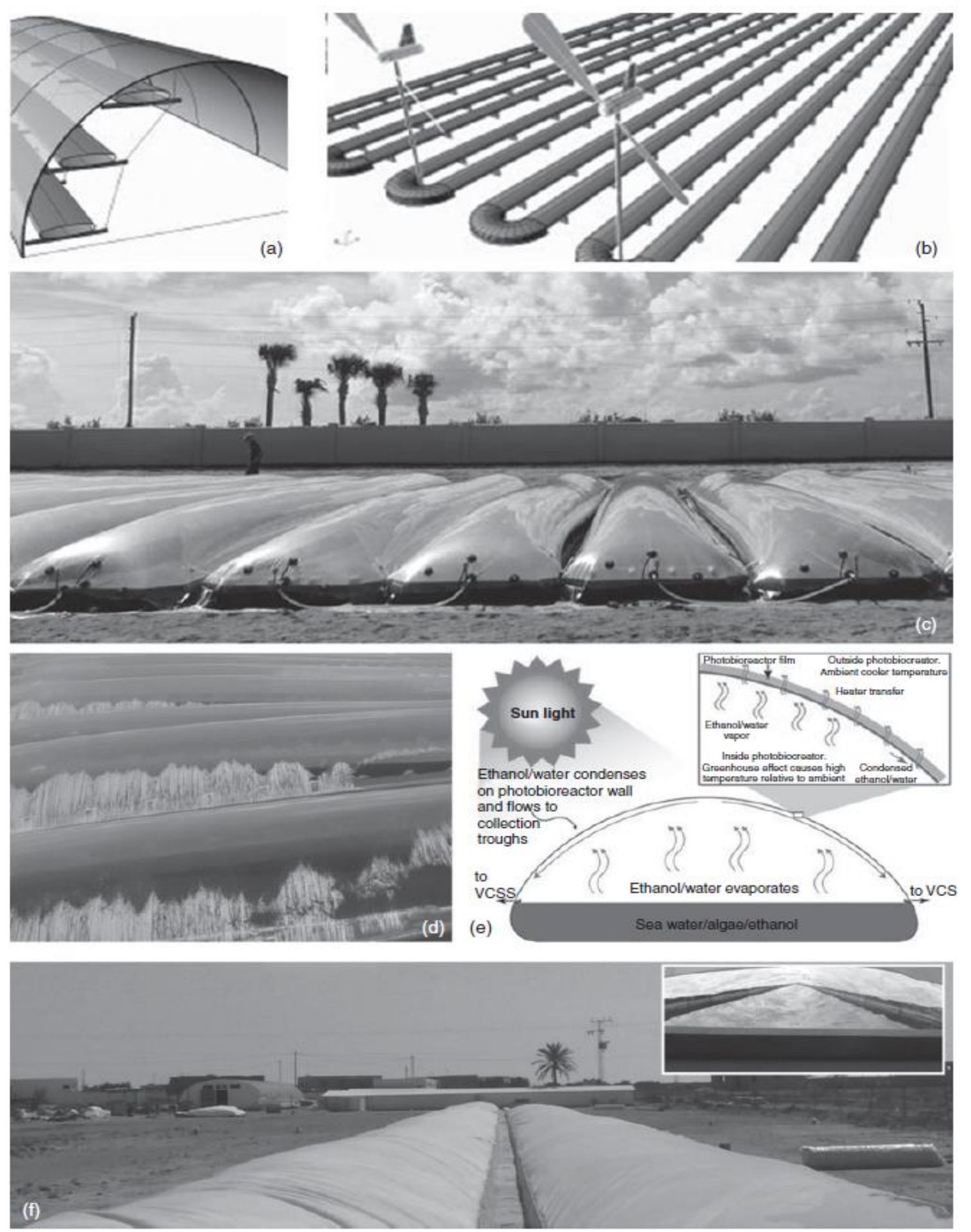

Figure 2.2. Hybrid systems. (a,b) Different configurations of a C-shaped fiberglass integrated open pond-PBR designed by Mottahedeh \& Tredici (2012); (c) pressurized polyethylene bags at Algenol BioFuels, Inc. (Florida, USA), for bioethanol production; (d) detail of the condensed water-ethanol vapor on the reactor wall (e) and scheme of the process; (f) Algae Tunnel system at AlgaeStream SA (France), the inset shows the interior of a tunnel (Zittelli et al., 2013) 


\subsubsection{Floating Systems}

Floating systems are PBRs deployed in bodies of water, which are anchored to a pier or mooring to prevent them from drifting away with waves or currents. These PBRs do not compete with the use of land, and if built close to urbanized areas (where little land is available), these PBRs can be sustained with nutrients from wastewaters and flue gases easily accessible in these areas.

The commercial exploitation of this technology still requires development and optimization. The first floating system was devised by Gudin in the 1980s, which could be thermoregulated by means of deflating its air-filled tubes and immersing them on a water pool (Tredici, 1999). Algasol Renewables SL (Mallorca, Spain) designed a floating system made of polyethylene bags, where temperature control and mixing is provided by wave action (Zittelli et al., 2013). A similar system has been designed by NASA (USA) scientists (Zittelli et al., 2013). The Energy Dynamics Laboratory of Utah State University Research Foundation developed a floating algae pond for oil production (Figure 2.3). Similar systems have been proposed by Muller Feuga \& Lemar (2011), Berzin et al. (2011), and Bussell (2010). Moreover, concepts of offshore algae farms, where biomass could be produced, treated and harvested in the open ocean have been patented by Albus et al., (2010), Bussell (2010), and Patel et al., (2011). However, no steps have been taken toward realization of an offshore commercial plant. Economic evaluation of the process needs to be done and structural resistance of the construction material against storms, waves and decay need to be tested (Zittelli et al., 2013). 

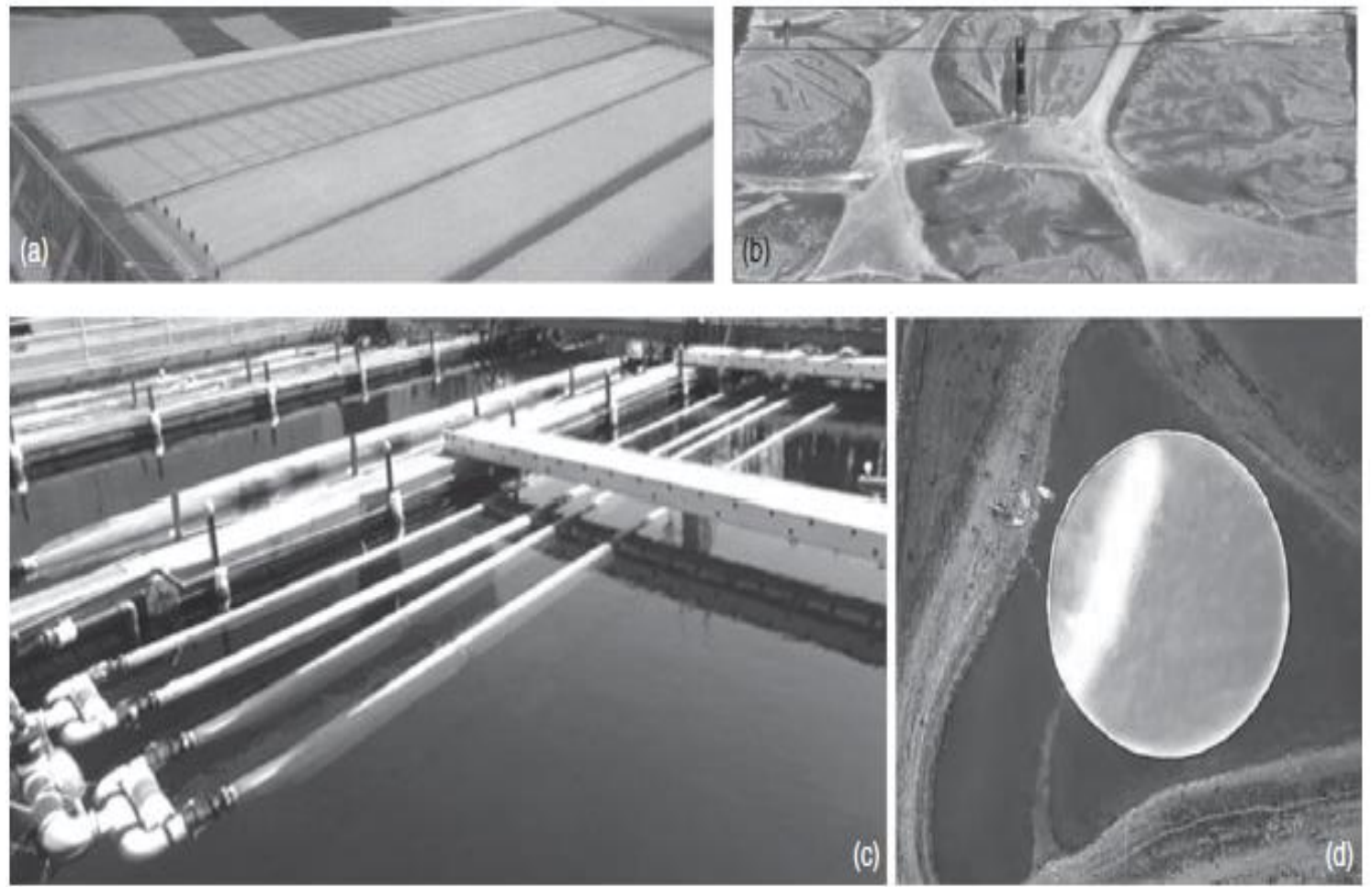

Figure 2.3. Floating Systems. (a) Floating tubular reactor designed by C. Gudin at Heliosynthesis in the 1980s; (b) floating PBR developed by Algasol Renewables SL (Spain); (c) low-density polyethylene tubular OMEGA (Offshore Membrane Enclosures for Growing Algae) photobioreactor developed by NASA (USA), at San Francisco OMEGA experimental site; (d) 0.4-ha algae floating pond developed at Utah State University Research Foundation and deployed on a wastewater pond at the Bingham Research Center in Utah, USA (Zittelli et al., 2013)

\subsubsection{Summary of Present Enterprises}

Table 2.3 describes the large-scale microalgal culture systems currently in use. This table depicts the principal applications of microalgae at present to be human nutrition, aquaculture and cosmetics. It should be noted that tabulations herein are of a generalized nature due to rapid developments in the microalgae industry. 
Table 2.3. Present state of large-scale microalgal production (Carvalho et al., 2006;

Spolaore et al., 2006)

\begin{tabular}{|c|c|c|c|c|c|}
\hline $\begin{array}{l}\text { Microalgal } \\
\text { species }\end{array}$ & $\begin{array}{l}\text { Annual } \\
\text { biomass } \\
\text { production } \\
\text { (Tons } \\
\text { DW)* } \\
\end{array}$ & Metabolite & Application & $\begin{array}{l}\text { Culturing } \\
\text { system }\end{array}$ & Location \\
\hline \multirow[t]{2}{*}{ Chlorella } & 2000 & Astaxanthin, & $\begin{array}{l}\text { Pigmenting } \\
\text { agent, human } \\
\text { nutrition, } \\
\text { aquaculture, } \\
\text { cosmetics }\end{array}$ & $\begin{array}{l}\text { Circular ponds } \\
\text { with rotating } \\
\text { arm }\end{array}$ & $\begin{array}{l}\text { Taiwan, } \\
\text { Japan, } \\
\text { U.S., } \\
\text { Thailand, } \\
\text { Germany }\end{array}$ \\
\hline & & ferrodoxin & Laboratory use & $\begin{array}{l}\text { Raceway } \\
\text { ponds, } \\
\text { heterotrophic } \\
\text { fermenters }\end{array}$ & $\begin{array}{l}\text { Taiwan, } \\
\text { Japan, } \\
\text { U.S., } \\
\text { Thailand, } \\
\text { Germany }\end{array}$ \\
\hline Dunaliella salina & 1200 & $\beta$-carotene & $\begin{array}{l}\text { Pigmenting } \\
\text { agent, human } \\
\text { nutrition, } \\
\text { cosmetics }\end{array}$ & $\begin{array}{l}\text { Extensive open } \\
\text { ponds, raceway } \\
\text { ponds }\end{array}$ & $\begin{array}{l}\text { Australia, } \\
\text { China, } \\
\text { India, } \\
\text { Chile, U.S., } \\
\text { Israel }\end{array}$ \\
\hline Arthrospira & & phycocyanin & Food coloring & $\begin{array}{l}\text { Extensive open } \\
\text { ponds, raceway } \\
\text { ponds }\end{array}$ & $\begin{array}{l}\text { Australia, } \\
\text { China, } \\
\text { India, } \\
\text { Chile, U.S., } \\
\text { Israel }\end{array}$ \\
\hline Arthrospira & 3000 & phycobiliproteins & $\begin{array}{l}\text { Human and } \\
\text { animal } \\
\text { nutrition, } \\
\text { cosmetics }\end{array}$ & Raceway ponds & $\begin{array}{l}\text { China, } \\
\text { India, U.S., } \\
\text { Myanmar, } \\
\text { Japan }\end{array}$ \\
\hline $\begin{array}{l}\text { Aphanizomenon } \\
\text { flos-aquae }\end{array}$ & 500 & & $\begin{array}{l}\text { Human } \\
\text { nutrition }\end{array}$ & & U.S. \\
\hline $\begin{array}{l}\text { Haematococcus } \\
\text { pluvialis }\end{array}$ & 300 & astaxanthin & $\begin{array}{l}\text { Pigmentin } \\
\text { agent, } \\
\text { aquaculture }\end{array}$ & $\begin{array}{l}\text { Extensive open } \\
\text { ponds, raceway } \\
\text { ponds }\end{array}$ & $\begin{array}{l}\text { U.S., India, } \\
\text { Israel }\end{array}$ \\
\hline $\begin{array}{l}\text { Crypthecodinium } \\
\text { cohnii }\end{array}$ & & $\begin{array}{l}\text { docosahexaenoic } \\
\text { acid }\end{array}$ & $\begin{array}{l}\text { functional food } \\
\text { additive }\end{array}$ & $\begin{array}{l}\text { Heterotrophic } \\
\text { fermenters }\end{array}$ & U.S. \\
\hline Shizochytrium & & $\begin{array}{l}\text { docosahexaenoic } \\
\text { acid }\end{array}$ & $\begin{array}{l}\text { functional food } \\
\text { additive }\end{array}$ & $\begin{array}{l}\text { Heterotrophic } \\
\text { fermenters }\end{array}$ & U.S. \\
\hline $\begin{array}{l}\text { Isochrysis } \\
\text { galbana }\end{array}$ & & $\begin{array}{l}\text { docosahexaenoic } \\
\text { acid }\end{array}$ & $\begin{array}{l}\text { functional food } \\
\text { additive }\end{array}$ & $\begin{array}{l}\text { Extensive open } \\
\text { ponds, raceway } \\
\text { ponds }\end{array}$ & U.S. \\
\hline
\end{tabular}

*Note: DW = Dry Weight

Table 2.4 summarizes companies and universities involved in the microalgae industry at the time of publication of this thesis project. The majority of these companies are startups and small firms in the field. Most experts working in the field agree that 50-100 $\mathrm{tha}^{-1}$ 
$\mathrm{yr}^{-1}$ is feasible, whereas more than that amount is not attainable at large scale with present strains and technologies (Zittelli et al., 2013). Nevertheless, some companies claim to be able to produce $300-600 \mathrm{tha}^{-1} \mathrm{yr}^{-1}$ and reach the maximum theoretical photosynthetic efficiency $(12.4 \%)$ and surpass the maximum of 5\% actually achieved outdoors (Zittelli et al., 2013). It is worth noting that these high values are estimates, while the low values are the actual results of experimentation (Zittelli et al., 2013).

In terms of reactor cost per square unit of occupied land area, comparison of different systems is difficult to illustrate since some companies report reactor cost with control and ancillary equipment included, while other companies report it as reactor alone (Zittelli et al., 2013). However, it appears that the microalgae industry could soon be able to produce algae in PBRs at costs similar or even lower than in open ponds on a "cost per mass of algae" basis (Lundquist pers. com., 2014). 
Table 2.4. Cultivation systems of start-ups and new small firms in the field (Zittelli

et al., 2013)

\begin{tabular}{|c|c|c|c|c|}
\hline Company/university & $\begin{array}{l}\text { Reactor type and } \\
\text { volume/land area }\end{array}$ & $\begin{array}{l}\text { Reactor cost } \\
\text { (in } 2013 € \text { ) }\end{array}$ & $\begin{array}{l}\text { Overall Areal } \\
\text { Productivity } \\
\left(\mathbf{t ~ h a}^{-1} \mathbf{y r}^{-1}\right)\end{array}$ & $\begin{array}{l}\text { Microalgal } \\
\text { species }\end{array}$ \\
\hline $\begin{array}{l}\text { University of Florence } \\
\text { (Italy) }\end{array}$ & $\begin{array}{l}\text { Advanced raceway } \\
\text { pond, } 500 \mathrm{~m}^{2}\end{array}$ & & & \\
\hline Institute of & Inclined pond, 700 & & $34^{\mathrm{b}}$ (Trebon) & Chlorella \\
\hline Microbiology, & $\mathrm{m}^{2}$ (Trebon); 100 & & $77^{\mathrm{b}}$ (Kalamata) & \\
\hline $\begin{array}{l}\text { Academy of Sciences } \\
\text { (Czech Republic) }\end{array}$ & $\mathrm{m}^{2}$ (Kalamata) & & & \\
\hline $\begin{array}{l}\text { AlgAmerica, LLC } \\
\text { (Pennsylvania, USA) }\end{array}$ & $\begin{array}{l}\text { Vertical Column, } \\
400 \mathrm{~L}\end{array}$ & $€ 852 \mathrm{PBR}^{-1}$ & & \\
\hline $\begin{array}{l}\text { University of Florence } \\
\text { (Italy) }\end{array}$ & $\begin{array}{l}\text { Annular vertical } \\
\text { column, } 120 \mathrm{~L}\end{array}$ & $\begin{array}{l}€ 1000 \mathrm{PBR}^{-1} \\
€ 5000 \mathrm{~m}^{-2} \\
\text { occupied area }\end{array}$ & $73^{\mathrm{b}, \mathrm{c}}$ & $\begin{array}{l}\text { Tetraselmis } \\
\text { suecica }\end{array}$ \\
\hline $\begin{array}{l}\text { University of Almeria } \\
\text { (Spain) }\end{array}$ & $\begin{array}{l}\text { Tubular horizontal } \\
\text { serpentine, } 4000 \mathrm{~L}\end{array}$ & & & $\begin{array}{l}\text { Scenedesmus } \\
\text { almeriensis }\end{array}$ \\
\hline $\begin{array}{l}\text { AlgaeLink NV (The } \\
\text { Netherlands) }\end{array}$ & $\begin{array}{l}\text { Tubular horizontal } \\
\text { serpentine PBR, } \\
97,000 \mathrm{~L}\end{array}$ & $\begin{array}{l}€ 160 \mathrm{~m}^{-2} \\
\text { occupied area }\end{array}$ & $\begin{array}{l}160^{\mathrm{a}} \text { (The } \\
\text { Netherlands) } \\
300^{\mathrm{a}} \\
\text { (Australia) }\end{array}$ & Tetraselmis \\
\hline $\begin{array}{l}\text { Institute of } \\
\text { Microbiology, } \\
\text { Academy of Sciences } \\
\text { (Czech Republic) }\end{array}$ & $\begin{array}{l}\text { Tubular Horizontal } \\
\text { serpentine, } 65 \mathrm{~L}\end{array}$ & $\begin{array}{l}€ 131 \mathrm{~m}^{-2} \\
\text { occupied area }\end{array}$ & $33^{\mathrm{b}}$ & Arthrospira \\
\hline $\begin{array}{l}\text { University of Almeria } \\
\text { (Spain) }\end{array}$ & $\begin{array}{l}\text { Tubular vertical } \\
\text { serpentine, } 2800 \mathrm{~L}\end{array}$ & & $73^{\mathrm{b}}$ & Nannochloropsis \\
\hline $\begin{array}{l}\text { Microphyt SAS } \\
\text { (France) }\end{array}$ & $\begin{array}{l}\text { Tubular vertical } \\
\text { serpentine, } 5400 \mathrm{~L}\end{array}$ & & $35^{\mathrm{b}}$ & $\begin{array}{l}\text { Neochloris } \\
\text { oleoabundans }\end{array}$ \\
\hline $\begin{array}{l}\text { GFT Corp. } \\
\text { (Massachusetts, USA) }\end{array}$ & $\begin{array}{l}\text { Triangular tubular } \\
\text { manifold airlift }\end{array}$ & & & \\
\hline $\begin{array}{l}\text { GFT Corp. } \\
\text { (Massachusetts, USA) }\end{array}$ & $\begin{array}{l}\text { 3D Matrix tubular } \\
\text { manifold }\end{array}$ & & $358^{\mathrm{b}}$ & \\
\hline $\begin{array}{l}\text { Subitec GmbH } \\
\text { (Germany) }\end{array}$ & $\begin{array}{l}\text { Vertical flat panel } \\
\text { (FPA), 180 L }\end{array}$ & $\begin{array}{l}€ 38 \mathrm{~m}^{-2} \\
\text { occupied area }\end{array}$ & $120^{\mathrm{a}}$ & \\
\hline $\begin{array}{l}\text { University of } \\
\text { Florence/F\&M Srl } \\
\text { (Italy) }\end{array}$ & $\begin{array}{l}\text { Flexible film } \\
\text { disposable panel } \\
\text { (GWP-I), } 800 \mathrm{~L}\end{array}$ & $\begin{array}{l}€ 50 \mathrm{~m}^{-2} \\
\text { occupied area }\end{array}$ & $40^{\mathrm{b}, \mathrm{c}}$ & Nannochloropsis \\
\hline $\begin{array}{l}\text { University of } \\
\text { Florence/F\&M Srl } \\
\text { (Italy) }\end{array}$ & $\begin{array}{l}\text { Flexible film } \\
\text { disposable panel } \\
\text { (GWP-II), } 350 \mathrm{~L}\end{array}$ & $\begin{array}{l}€ 25 \mathrm{~m}^{-2} \\
\text { occupied area }\end{array}$ & $54^{\mathrm{b}, \mathrm{c}}$ & $\begin{array}{l}\text { Tetraselmis } \\
\text { suecica }\end{array}$ \\
\hline $\begin{array}{l}\text { Archimede Ricerche Srl } \\
\text { (Italy) }\end{array}$ & $\begin{array}{l}\text { Fexible film } \\
\text { disposable panel } \\
\text { (GWP-I), } \\
40,000 \mathrm{~L}\end{array}$ & & $20^{\mathrm{b}, \mathrm{c}}$ & $\begin{array}{l}\text { Nannochloropsis, } \\
\text { Isochrysis, } \\
\text { Tetraselmis }\end{array}$ \\
\hline Vertigro Algae & Vertical flexible & & $600^{\mathrm{a}}$ & \\
\hline $\begin{array}{l}\text { Technologies, LLC } \\
\text { (Texas, USA) }\end{array}$ & film panel & & & \\
\hline $\begin{array}{l}\text { Proviron Holding NV } \\
\text { (Belgium) }\end{array}$ & $\begin{array}{l}\text { Vertical flexible } \\
\text { film panel } \\
\text { (ProviAPT) }\end{array}$ & $\begin{array}{l}€ 10 \mathrm{~m}^{-2} \\
\text { occupied area }\end{array}$ & & \\
\hline
\end{tabular}


Table 2.4. (Continued)

\begin{tabular}{|c|c|c|c|c|}
\hline Company/university & $\begin{array}{l}\text { Reactor type and } \\
\text { volume/ land area }\end{array}$ & $\begin{array}{l}\text { Reactor cost } \\
\text { (in } 2013 € \text { ) }\end{array}$ & $\begin{array}{l}\text { Overall areal } \\
\text { productivity } \\
\left(\mathbf{t ~ h a}^{-1} \mathrm{yr}^{-1}\right)\end{array}$ & $\begin{array}{l}\text { Microalgal } \\
\text { species }\end{array}$ \\
\hline Phytolutions (Germany) & $\begin{array}{l}\text { Vertical flexible } \\
\text { film panel, } \\
25,000 \mathrm{~L}\end{array}$ & $\begin{array}{l}€ 15 \mathrm{~m}^{-2} \\
\text { occupied area }\end{array}$ & $80-120^{\mathrm{a}}$ & \\
\hline $\begin{array}{l}\text { Photon8, Inc. (Texas, } \\
\text { USA) }\end{array}$ & $\begin{array}{l}\text { Horizontal flexible } \\
\text { film panel (Parallel } \\
\text { Film Reactor) }\end{array}$ & $\begin{array}{l}€ 8 \mathrm{~m}^{-2} \\
\text { occupied area }\end{array}$ & $182^{\mathrm{b}}$ & \\
\hline $\begin{array}{l}\text { AlgAmerica, LLC } \\
\text { (Pennsylvania, USA) }\end{array}$ & $\begin{array}{l}\text { Horizontal flexible } \\
\text { film panel, } 2600 \mathrm{~L}\end{array}$ & $\begin{array}{l}€ 24 \mathrm{~m}^{-2} \\
\text { occupied area }\end{array}$ & & \\
\hline & $\begin{array}{l}\text { Hybrid trough } \\
\text { system }\left(\text { Simgae }{ }^{\mathrm{TM}}\right)\end{array}$ & $\begin{array}{l}€ 4 \mathrm{~m}^{-2} \\
\text { occupied area }\end{array}$ & $50^{\mathrm{a}}$ & \\
\hline $\begin{array}{l}\text { Phycobiosciences, Inc. } \\
\text { (Arizona, USA) }\end{array}$ & $\begin{array}{l}\text { Hybrid trough } \\
\text { system (Super } \\
\text { Trough), } 133,000 \mathrm{~L}\end{array}$ & $\begin{array}{l}€ 47 \mathrm{~m}^{-2} \\
\text { occupied area }\end{array}$ & & \\
\hline $\begin{array}{l}\text { MBD Energy Ltd } \\
\text { (Australia) } \\
\text { Algenol Biofuels, Inc. } \\
\text { (Florida, USA) }\end{array}$ & $\begin{array}{l}\text { Hybrid horizontal- } \\
\text { bag system (BAGS) } \\
\text { Hybrid horizontal- } \\
\text { bag system, } 4500 \mathrm{~L}\end{array}$ & & & \\
\hline $\begin{array}{l}\text { AlgaeStream SA } \\
\text { (France) }\end{array}$ & $\begin{array}{l}\text { Hybrid system } \\
\text { (Algae Tunnel), } \\
\text { 200,000-300,000 L }\end{array}$ & $\begin{array}{l}€ 50 \mathrm{~m}^{-2} \\
\text { occupied area }\end{array}$ & $91^{\mathrm{b}}$ & Arthrospira sp. \\
\hline $\begin{array}{l}\text { Algasol Renewables SL } \\
\text { (Spain) }\end{array}$ & $\begin{array}{l}\text { Floating } \\
\text { photobioreactors } \\
\left(100 \mathrm{~m}^{2}, 5000 \mathrm{~L} ;\right. \\
\& 1 \mathrm{ha}, 500,000 \mathrm{~L})\end{array}$ & & $\begin{array}{l}105^{\mathrm{b}} \\
130^{\mathrm{a}}\end{array}$ & $\begin{array}{l}\text { Chlorella } \\
\text { Vulgaris, } \\
\text { Neochloris } \\
\text { oleoabundans }\end{array}$ \\
\hline $\begin{array}{l}\text { Energy Dynamics } \\
\text { Laboratory (USA) }\end{array}$ & Floating pond & & $69^{\mathrm{b}}$ & \\
\hline Ohio University’s Coal & Film membrane & & $108^{\mathrm{a}}$ & Chlorogloeopsis \\
\hline $\begin{array}{l}\text { Research Center (Ohio, } \\
\text { USA) }\end{array}$ & $\begin{array}{l}\text { PBR for attached } \\
\text { growth (CRF) }\end{array}$ & & & sp. \\
\hline $\begin{array}{l}\text { SBAE Industries NV } \\
\text { (Belgium) }\end{array}$ & $\begin{array}{l}\text { Film PBR for } \\
\text { attached growth } \\
\text { (DIAFORCE } \\
200 \mathrm{~m}^{2} \text { ), }\end{array}$ & & $100^{\mathrm{b}}$ & $\begin{array}{l}\text { Diatom } \\
\text { polyculture }\end{array}$ \\
\hline $\begin{array}{l}\text { University of } \\
\text { Florence/CNR-ISE } \\
\text { (Italy) }\end{array}$ & $\begin{array}{l}\text { Combined process } \\
\text { (pond + panel PBR) }\end{array}$ & & $32^{\mathrm{b}}$ & $\begin{array}{l}\text { Arthrospira } \\
\text { platensis }\end{array}$ \\
\hline $\begin{array}{l}\text { University of Hawaii } \\
\text { (USA) }\end{array}$ & $\begin{array}{l}\text { Combined process } \\
\text { (pond + horizontal } \\
\text { serpentine PBR), } \\
50,000 \mathrm{~L}+ \\
25,000 \mathrm{~L}\end{array}$ & & $38^{\mathrm{b}}$ & $\begin{array}{l}\text { Haematococcus } \\
\text { pluvialis }\end{array}$ \\
\hline $\begin{array}{l}\text { HR BioPetroleum, Inc., } \\
\text { now Cellana (Hawaii, } \\
\text { USA) }\end{array}$ & $\begin{array}{l}\text { Combined process } \\
\text { (pond }+ \\
\text { horizontal serpentine } \\
\text { PBR) }\end{array}$ & & $148^{\mathrm{a}}$ & \\
\hline $\begin{array}{l}\text { Solix Biosystems, Inc. } \\
\text { (Colorado, USA) }\end{array}$ & $\begin{array}{l}\text { Vertical flexible } \\
\text { film panel (AGS- } \\
4000 \text { ) }\end{array}$ & $\begin{array}{l}€ 3000 \mathrm{~m}^{-2} \\
\text { occupied area }\end{array}$ & & \\
\hline
\end{tabular}




\subsection{Design Criteria for Photobioreactors}

Design criteria for PBRs should be aimed at increasing volumetric and areal productivity, photosynthetic efficiency and cost-effectiveness of algal growth (Tredici, 2004). These criteria are described in the subsections below.

\subsubsection{Surface-to-Volume Ratio (S/V)}

According to Tredici (2004), the ratio between illuminated surface area of reactor photostage and volume of the reactor $(\mathrm{S} / \mathrm{V})$ plays an important role in PBR design. Tredici (2004) argues that generally higher S/V ratios will give higher cell concentrations and higher volumetric productivities in a PBR. Higher cell concentrations decrease the cost of harvesting, medium preparation and culture handling. However, too high of an S/V ratio

will cause a PBR to suffer from excessive changes in oxygen evolution, $\mathrm{CO}_{2}$ absorption, nutrient depletion and metabolite excretion, which in turn has negative long-term effects on the culture. Moreover, a PBR with an excessively high S/V ratio may become less efficient when scaled up to industrial size. Optimum S/V ratios to achieve maximum illuminated surface productivity have been reported, but it depends on the incident light intensity. Tubular PBRs are preferred when one of the design goals is to have nearoptimum $\mathrm{S} / \mathrm{V}$ ratios since this parameter can be optimized by changing the diameter of the tubes.

\subsubsection{Orientation and Inclination}

Unlike systems placed parallel to the ground, vertical systems can be oriented and tilted at different angles with respect to solar irradiation to maximize the use of sunlight (Tredici, 2004). While no great influence has been found on inclination of a PBR with 
respect to productivity at low latitudes, it's been shown that inclination significantly affects productivity at higher latitudes (Tredici, 2004). Nevertheless, PBRs facing south and tilted at an angle to intercept maximum solar irradiation (sun-oriented systems) generally achieve higher volumetric productivities (Tredici, 2004).

\subsubsection{Gas Exchange}

For a high photosynthesis rate in an algal culture, the $\mathrm{CO}_{2} / \mathrm{O}_{2}$ balance has to be adjusted in a way that the prime carboxylating enzyme in algae, rubisco, furnishes $\mathrm{CO}_{2}$ for the Calvin cycle but does not use $\mathrm{O}_{2}$ for photorespiration (Pulz, 2001). Hence, in algal cultures sufficient $\mathrm{CO}_{2}$ must be available while evolved $\mathrm{O}_{2}$ has to be removed before reaching inhibitory concentrations (Pulz, 2001). This adjustment is done by means of stripping $\mathrm{O}_{2}$ with air bubbling out of the culture, monitoring the $\mathrm{pH}$ of the culture and controlling the culture $\mathrm{pH}$ through on-demand $\mathrm{CO}_{2}$ injection.

Accumulation of photosynthetically-generated oxygen has been one of the major limiting factors of PBR scale up, especially with tubular reactors (Weissman et al., 1988). Concentrations of dissolved oxygen above air saturation are typically harmful to many autotrophs, and toxic concentrations of dissolved oxygen can easily be reached in tubular reactors with too high of an S/V ratio and severely inhibit algal growth; given the rate of $\mathrm{O}_{2}$ production and its low solubility (Weissman et al., 1988). Manifold systems, vertical columns, and flat panels mixed by air-bubbling offer a significant advantage in this issue because they provide a shorter oxygen path for mass transfer of oxygen out of solution (Sierra et al., 2008). 
Carbon dioxide supply is a major component of production costs and is the usual carbon source for photosynthetic growth of microalgae (Tredici, 2004). The dissolved carbon dioxide is normally in a pH-dependent equilibrium state with carbonate and bicarbonate species, thus, the total inorganic carbon concentration and the dissolved carbon dioxide in an algal culture are interrelated (Chisti et al., 1999). The $\mathrm{pH}$ variation in culture is mainly due to consumption of carbon dioxide, although variations in $\mathrm{pH}$ due to consumption of other nutrients and/or degradation of the excreted metabolites occur (Chisti et al., 1999). Loss of dissolved carbon dioxide due to uptake into algal cells is partly compensated by regeneration from carbonates and bicarbonates, thus, carbon dioxide uptake is accompanied by changes in $\mathrm{pH}$ (Chisti et al, 1999). Injection of $\mathrm{CO}_{2}$ as minute bubbles at the beginning of a tubular solar receiver has proven to be an efficient means of supplying $\mathrm{CO}_{2}$ to tubular reactors (Chisti et al., 1999).

\subsubsection{Mixing}

Choosing the appropriate type of device to mix the culture is essential in PBR design (Tredici, 2004). Mixing is necessary to prevent cells from settling, avoid thermal stratification, break down diffusion gradients, distribute nutrients in the medium, provide adequate supply of $\mathrm{CO}_{2}$ and oxygen removal, and ensure proper irradiance to all the cells in the culture (Weissman et al., 1988). Generally, cultures of fragile microorganisms cannot be mixed with mechanical stirrers, such as the ones used in fermenter-type bioreactors, due to high hydrodynamic shear stress the stirrer applies to cells (Carvalho, 2006). Hydrodynamic shear stress is when certain overcritical hydrodynamic load is applied to microorganisms and cell damage occurs (Bronnenmeier and Markl, 1982). 
Airlift systems are preferred for fragile microorganisms since they cause lower hydrodynamic shear stress to cells than pumps (Tredici, 2004).

\subsubsection{Circulation}

Culture velocity in tubular reactors is usually between 0.2 and $0.3 \mathrm{~m} \mathrm{~s}^{-1}$, and is what determines mass transfer capacity and turbulence (Tredici, 2004). Culture velocity partly affects adequate exchange of gasses because it determines the amount of time certain bulk of the fluid in the tubes of a tubular reactor reaches the gas-exchange devise for stripping the photosynthetically-generated oxygen (Tredici, 2004). Systems for culture circulation can be a major photobioreactor design issue in terms of both capital cost and power consumption (Weissman et al., 1988).

Finding an optimum circulation velocity for the culture is essential in a tubular reactor. The power requirement for circulation is very dependent on culture velocity, thus, minimizing velocity will reduce power consumption (Norsker et al., 2012). However, to low of a circulation velocity will eliminate turbulence necessary for mixing to occur in the tubes (Geankoplis, 2003).

The choice of circulation device and circulation velocity also depend on the type of microorganism to be cultured (Tredici, 2004). Shear-sensitive microalgae can only withstand certain magnitudes of turbulence (Sierra et al., 2008). Also, Tredici (2004) argues that cultures of fragile microorganisms cannot be circulated by pumps. Bronnenmeier and Markl (1982) present an example regarding the flow around a turbine blade (Figure 2.4). A stagnant area is found at the front side and a strongly accelerating stream is produced at the tips of the blade. At the back of the blade there is a low- 
pressure zone with eddies moving away from it. In this stream two destructive mechanisms are possible: (1) rapid pressure change over the blade accompanied by acceleration of the microorganisms and (2) high shear stresses at the turbine tips. As a result of overcritical hydrodynamic load, damage of sensitive microorganisms will occur. Thus, circulation with air blowers or air compressors is preferred for fragile microorganisms since they cause lower hydrodynamic shear stress than pumps (Tredici, 2004).

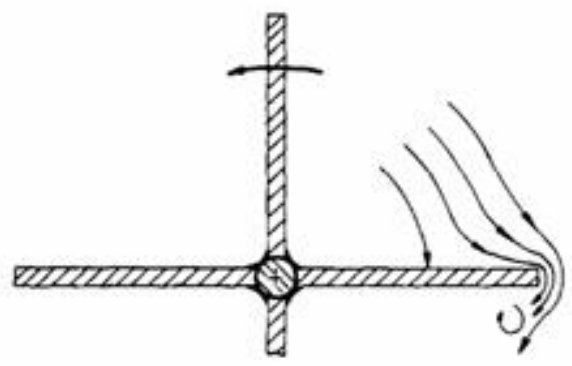

Figure 2.4. Flow around a turbine blade (Bronnenmeier \& Markl, 1982)

\subsubsection{Temperature Control}

The optimal temperature range for growth differs with type of microorganism (Tredici, 2004). While open ponds are limited by low temperatures in the morning, PBRs generally require cooling at midday, thus, several methods are used to deal with thermoregulation of PBRs (Tredici, 2004). Shading, immersion in a water bath and water spraying are the most common methods to avoid outdoor PBRs from overheating (Tredici, 2004). Shading, however, requires up to $80 \%$ of the photo-stage portion of the PBR be covered, and this normally causes a decrease in productivity (Tredici, 2004). The costeffectiveness of cooling by immersion in a water bath is rather doubtful (Tredici, 2004). 
Cooling by water spraying is more cost effective and reliable in dry climates since it requires a smaller water footprint as compared to cooling by water immersion (Tredici, 2004). The reported literature suggests that evaporative cooling is more economic than heat exchangers (Tredici, 2004).

\subsubsection{Water Footprint}

In a techno-economic, comprehensive analysis of autotrophic algae cultivation in an open pond and a tubular PBR aimed at establishing baseline economics for biofuel production, Davis et al. (2011) demonstrated the advantage of tubular PBRs over open ponds with respect to water use. In this study, having both systems the same lipid production (10 MM gal $\mathrm{yr}^{-1}$ ) as a basis, it was found that the tubular PBR scenario required $30 \%$ of the net water demand for the open pond scenario. The study also found that $57 \%$ of the net water demand for the open pond scenario was lost to evaporation, and $43 \%$ lost due to discharge to a water-body downstream or to an off-site treatment facility. In the tubular PBR case, $83 \%$ of the net water demand was lost due to evaporative cooling of the PBR, and $17 \%$ lost due to discharge to a water-body downstream or to an off-site treatment facility. This analysis was performed using Aspen Plus software, assumptions based off of vendor quotes, prior literature studies and standard engineering estimates to evaluate

all capital and operating costs in order to stablish an overall cost of production value for both the open pond scenario and the tubular PBR scenario.

\subsubsection{Construction Materials for PBRs}

The choice of construction material for the photo-stage is critical in PBR engineering (Tredici, 2004). Materials for a PBR must be non-toxic to microorganisms, must have 
high mechanical strength, high durability (resistance to weathering), high translucence, chemical stability, low cost and ease of cleaning (when biofouling is an operational issue) (Tredici, 2004).

\subsection{Scale Up of Photobioreactors}

The level of difficulty of scaling up a PBR is proportional to the size of the container and to the tolerances of the microorganism (Olaizola, 2000). When scaling up a PBR, illumination, gas exchange, temperature control and mixing regime need to be taken into consideration (Olaizola, 2003). No universal systematic scale up procedure exists for PBRs (Chisti et al., 1999). Non-tubular PBR designs, such as airlift columns, vertical sleeves and flat panels, generally face serious limitations when their volume exceeds 50$100 \mathrm{~L}$, since beyond this volume light energy is excessively diluted and gas transfer becomes limited (Pulz, 2001).

PBRs can be scaled up by multiplication of identical modules or by increasing the length and diameter of tubes; in the case of tubular PBRs (Chisti et al., 1999). However, simulations based on mass-transfer models with tubular reactors suggest that increasing the tube length at constant diameter can adversely change culture $\mathrm{pH}$, dissolved oxygen and $\mathrm{CO}_{2}$ absorption significantly due to increasing concentration gradients along the tube lenght (Chisti et al, 1999). For these reasons flat panels, manifold and airlift-column PBRs are seen as attractive designs (Tredici, 2004). The main drawback with these types of PBRs is the capital cost, which comes from the fact that numerous modules need to be built for a commercial plant. General recommendations as to maximum possible scales of tubular PBRs have been published. Chisti et al. (1999) and Molina Grima et al. (2000) 
recommended to use a pipe diameter of no more than $0.1 \mathrm{~m}$, and a continuous tube length of about $80 \mathrm{~m}$ with a flow velocity of 0.3 to $0.5 \mathrm{~m} \mathrm{~s}^{-1}$. Multiple parallel run tubes originating and ending in common headers are apparently the best way to accommodate higher flows and volumes (Chisti et al., 1999).

\subsection{Productivity in Photobioreactors}

Comparison between different types of PBRs and open ponds is not an easy task, because their evaluations depend on many factors (Tredici, 2004). Table 2.4 gives an idea of vastly different overall areal productivities experienced in facilities worldwide. Principal factors contributing to differences in calculated productivity are reactor design, whether the culture is being grown indoors or outdoors, the type of algal species chosen and the method adopted to compute productivity (Tredici, 2004).

\subsubsection{Methods of Evaluation and Comparison}

Algal culture performance is often illustrated by its specific growth rate, but what is often ignored is that high specific growth rates do not necessarily mean high productivities. The specific growth rate $(\mu)$ is defined as the increase in biomass per unit time and per unit biomass, and is one of the two variables needed to determine productivity (Zittelli et al., 2013). Productivity $(P)$ is calculated by multiplying the specific growth rate by the actual amount of biomass $(X)$ that is increasing at that specific rate $(P=\mu X)$ (Zittelli et al., 2013). Thus, productivity at steady state (also known as exponential or balanced growth phase) can be calculated as follows (Shuler \& Kargi, 2002):

$$
P=\mu X=\left(\frac{1}{X} \frac{d X}{d t}\right) X=\frac{d X}{d t}=\frac{\text { Change in unit biomass }}{\text { Change in unit time }}
$$


Table 2.5 shows how four parameters are commonly used to evaluate productivity in PBRs: volumetric productivity (VP), areal productivity (AP), illuminated surface productivity (ISP), and overall areal productivity (OAP).

Table 2.5. Recording microalgal productivities in PBRs and ponds (Tredici, 2004)

\begin{tabular}{lll}
\hline Biomass productivity & Units & Basis for calculation \\
\hline Volumetric productivity (VP) & $\mathrm{g}^{-1} \mathrm{~d}^{-1}$ & Reactor working volume \\
Illuminated surface productivity (ISP) & $\mathrm{g} \mathrm{m}^{-2} \mathrm{~d}^{-1}$ & Illuminated surface area of the reactor photo-stage \\
Areal productivity (AP) & $\mathrm{g} \mathrm{m}^{-2} \mathrm{~d}^{-1}$ & $\begin{array}{l}\text { Ground area occupied by reactor volume only } \\
\text { Overall areal productivity (OAP) }\end{array}$ \\
$\mathrm{g} \mathrm{m}^{-2} \mathrm{~d}^{-1}$ & $\begin{array}{l}\text { Ground area including the distance between } \\
\text { reactors or tubes (empty space), arranged } \\
\text { horizontally* or vertically** }\end{array}$ \\
& &
\end{tabular}

*Small peripheral effects

**Large peripheral effects

VP is a key parameter that illustrates how efficiently the unit volume of the reactor is used and is dependent on the $\mathrm{S} / \mathrm{V}$ ratio; the higher the $\mathrm{S} / \mathrm{V}$ ratio, the higher the VP of the PBR (Tredici, 2004).

Care should be taken when using AP and ISP to evaluate the productivity of a PBR. For calculation of the ISP in vertical systems the entire surface of the tubing system must be taken into account (Tredici, 2004). And when calculating the ISP for horizontal systems only the illuminated side of the reactor is taken into account. The AP and ISP for ponds, horizontal and near-horizontal flat reactors normally coincide because the occupied ground area that these reactors occupy is the same as their illuminated surface area (Tredici, 2004). In the case of horizontal tubular reactors with tubes in contact with each other, the illuminated surface area is generally 1.57 times the occupied ground area, so ISP will always be lower than AP for PBRs (Tredici, 2004). 
When it comes to evaluating the productivity of horizontal tubular reactors with empty space between contiguous tubes, and vertical and highly inclined systems, productivity calculation is more difficult unless a forth parameter for measuring productivity is introduced: the overall areal productivity (OAP) (Tredici, 2004). When evaluating productivity in reactors with empty space between contiguous tubes, and vertical and highly inclined systems, the OAP (expressed in $\mathrm{g} \mathrm{m}^{-2} \mathrm{~d}^{-1}$ ) must be calculated. The OAP is defined as the productivity obtained from the sum of the ground areas (including empty spaces) that occupy the units that constitute a plant (Tredici, 2004). The OAP has greater meaning for scale-up, for comparison between different kinds of reactors, and comparison of PBRs to ponds (Tredici, 2004). AP for vertical systems is a meaningless parameter and should always be avoided because length (or height) of the reactor is not taken into account compared to when calculating the AP for horizontal systems (Tredici, 2004). Hence, OAP is the version of areal productivity used consistently and exclusively in this thesis project.

When scaling up by multiplication of modules, it must be noted that productivities calculated using a single unit may not be representative of their productivity in a fullscale system. When scaling up by setting up a number of units, the output will be influenced by the distance separating the several units in the field (Tredici, 2004). In this case, an adequate number of units must be set up and operated and the OAP computed on the basis of the whole ground area occupied by all the units (including empty spaces), carefully considering peripheral effects (Tredici, 2004). Another common error when scaling up involves using data from short-term experiments to extrapolate to longer time 
periods (such as a year), when climatic conditions show an obvious seasonal variation (Zittelli et al., 2013).

Extrapolating productivity from pilot-scale PBRs is not straightforward because of edge or peripheral effects. Small PBR modules have more or their perimeter reactor surface area unshaded by adjacent tubes than would large groups of modules. Productivity could be overestimated in pilot-scale PBRs due to this effect (Lundquist, pers. Com., 2014).

Published year-round productivity data from PBRs are scarce, more data being available from open ponds. Maximum OAPs for short periods under optimum conditions have been reported to be in the range of 30-40 $\mathrm{g} \mathrm{m}^{-2} \mathrm{~d}^{-1}\left(120-160 \mathrm{tha}^{-1} \mathrm{yr}^{-1}\right)$ for both PBRs and open ponds (Zittelli et al., 2013). Typical OAPs obtained in pilot-scale, outdoors, yearround experiments range between 20 to $50 \mathrm{tha}^{-1} \mathrm{yr}^{-1}$ in PBRs and 20 to $80 \mathrm{tha}^{-1} \mathrm{yr}^{-1}$ in open ponds. OAPs in commercial open ponds normally do not exceed $30 \mathrm{tha}^{-1} \mathrm{yr}^{-1}$ (Zittelli et al., 2013). But this may be due to the stressful selective media used for several major commercial algae types (Lundquist, pers. com., 2014). Higher values should be considered overestimates unless supported by data from well-organized long-term experiments (Zittelli et al., 2013). Most experts agree that OAPs of $80 \mathrm{tha}^{-1} \mathrm{yr}^{-1}$, which is the range of high yield attained with $\mathrm{C} 4$ crops in the tropics, must be considered as the maximum achievable productivity with microalgae at large scale (Zittelli et al., 2013). It is this number that should be used as the best case for determining the feasibility of any algae venture, and any number higher would require genetically engineered strains capable of counteracting photosaturation and photoinhibition (Zittelli et al., 2013). 
Another parameter useful in determining the microalgae culture performance and comparing different culture systems in different locations is the photosynthetic efficiency (PE). The theoretical maximum PE for microalgae has been reported to be $12.4 \%$ (based on PAR), but PE values higher than 4-5\% (based on PAR) are rarely reached under actual outdoor conditions (Tredici, 2010). Also, a high PE is not necessarily associated with a high productivity since PE is normally higher at low irradiances (Zittelli et al., 2013). For example, during winter, in light-limited cultures, PE increases in response to lower irradiance, but this comes with a decrease in productivity (Zittelli et al., 2013).

The performance of a PBR can also be evaluated from an economic point of view, providing the cost of biomass production in the system that is being considered. Nevertheless, an economic analysis depends on the details of the algae and construction site (Tredici, 2004). For example, downstream processing and labor costs are some of the operating costs that change from site to site, and thus, will change final cost of the biomass (Tredici, 2004). Thus, production cost analysis should be based on the final cost of the biomass. Capital cost on the other hand is mainly a function of culture volume since cost of construction materials depend on size of the reactor and bigger land area required does not necessarily mean bigger volume (Acien et al., 2010). Thus, for PBRs, capital cost analysis should be done on a volume basis and not on an areal basis.

Another method to evaluate PBRs has been proposed by Pegallapati et al. (2014) to assess the energy efficiency of a cultivation process. Pegallapati et al. (2014) proposed the specific biomass productivity $(\mathrm{SBP}), P_{B} / E_{C}$ (in milligrams per kilojoule, $\mathrm{mg} \mathrm{kJ}^{-1}$ ), defined as the volumetric biomass productivity, $P_{B}\left(\mathrm{~g} \mathrm{~L}^{-1} \mathrm{~d}^{-1}\right)$ per energy input, $E_{C}\left(\mathrm{~W} \mathrm{~L}^{-}\right.$ $\left.{ }^{1}\right)$. Energy input, $E_{C}$, for cultivation includes that required for culture circulation, gas 
exchange, heating, cooling and illumination (Pegallapati et al., 2014). Energy input due to illumination is not a concern when growing algae outdoors, and energy required for heating or cooling is negligible compared to the energy required for culture circulation and gas exchange (Hulatt \& Thomas, 2011; Pegallapati et al., 2014). Optimal circulation and gas exchange is required to control growth conditions, such as nutrient supply and light utilization, which depend on the algal strain being grown (Pegallapati et al., 2014). Thus, energy input calculations should be focused on circulation and gas exchange and $P_{B} / E_{C}$ is a parameter that should be taken into consideration in process optimization and PBR design (Pegallapati et al., 2014).

\subsection{Types of PBRs and Early Attempts at Cultivation of Microalgae}

Over the past 60 years much research has been done about the effects of photobioreactor configuration on the growth of algae. The most common types of PBRs are the following: tubular, flat plate, airlift column, sleeves, fermenter-type and attached-growth PBRs. Each are described below.

\subsubsection{Tubular PBRs}

In the case of a relatively large transparent tank for growing algae, the cells pick up light while they are close to the tank wall, and at this time photosynthesis occurs with the $\mathrm{CO}_{2}$ and nutrients (Chisti, 2001). But once the cells move away from the tank wall and into the center of the tank the photosynthesis stops because light cannot penetrate such distances through the algae culture (Chisti, 2001). Thus, algal growth is limited in such a scenario. To work around this problem, tubular PBRs are built with relatively narrow diameter tubes that allow light to penetrate to the center of the tube (Chisti, 2001). This way the surface area for photosynthesis is maximized and the ratio of light to dark area is 
much greater than in a large transparent tank (Chisti, 2001). However, because there is still a need to add nutrients and remove photosynthetically-generated oxygen, the narrow tubing (which normally ranges 4-12 inches) is usually connected to a large-diameter airlift column and the algae is constantly recirculated around the narrow tubing and the airlift column (Chisti, 2001).

Tubular bioreactors are the predominant PBRs used for outdoor cultivation and there is a significant amount of published literature depicting its outstanding efficiency for growing algae (Oilgae, 2013). Tubular PBRs can be subcategorized in the following way: serpentine, manifold, helical and fence arrangement. These designs are described below.

\subsubsection{Serpentine PBRs}

Serpentine PBRs are made of several transparent narrow tubes arranged (in a 2D fashion) either horizontally (parallel to the ground) or vertically (Figure 2.5). The tubes are connected by U-bends to form a flat loop, and circulation is provided by a pump (Tredici, 2004). Nutrient addition and gas exchange normally occur in an airlift column; where the airlift system is located (Tredici, 2004). The narrow-tubing section of the system is called the photo-stage or solar receiver and the vessel where nutrient addition and gas transfer occurs is called the gas exchanger (Tredici, 2004). 


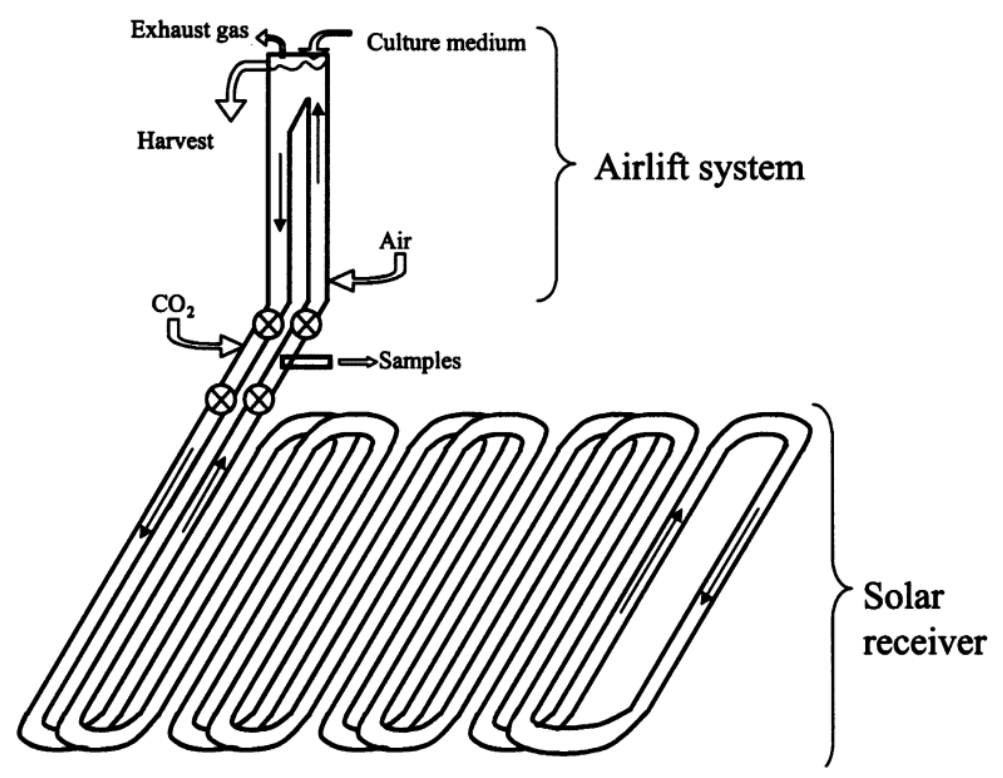

Figure 2.5. A horizontal tubular photobioreactor (Chisti, 2001)

The original design was created by Tamiya and coworkers with a 40-L reactor (Tamiya et al., 1953). This was followed by the development of a $56-\mathrm{m}^{2}$ horizontal serpentine system at the Massachusetts Institute of Technology (Tredici, 2004). The system built at MIT is considered to be the first pilot plant for microalgae production (Tredici, 2004).

Following initial studies in the 1950s, little work was done on serpentine systems for several decades. An exception is the work of Setlik in Czechoslovakia (Setlik et al, 1967). In the 1980s, Jüttner in Germany (Jüttner, 1982), Gudin and co-workers in France (Chaumont et al., 1988, Chaumont 1993), Pirt and co-workers in the UK (Pirt et al., 1983), and Florenzano and co-workers in Italy (Torzillo et al., 1986) revived incentives to study serpentine systems. In the 1990s, Molina Grima and co-workers in Spain (Molina Grima, 1999) developed reactors of the type developed by Gudin (Figure 2.6.a), and Torzillo et al. (1993) developed and experimented with a 240-L two-plane serpentine 
reactor (Figure 2.6.b) and was able to achieve a maximum areal productivity of $28 \mathrm{~g} \mathrm{~m}^{-}$ ${ }^{2} \mathrm{~d}^{-1}$. The photo-stage in these systems are submerged in a pond of water for thermoregulation, but the cost of such operation limits its applications (Tredici, 2004). Thus, microalgae species with relatively high temperature optima are more desirable for use with this type of system (Tredici, 2004).

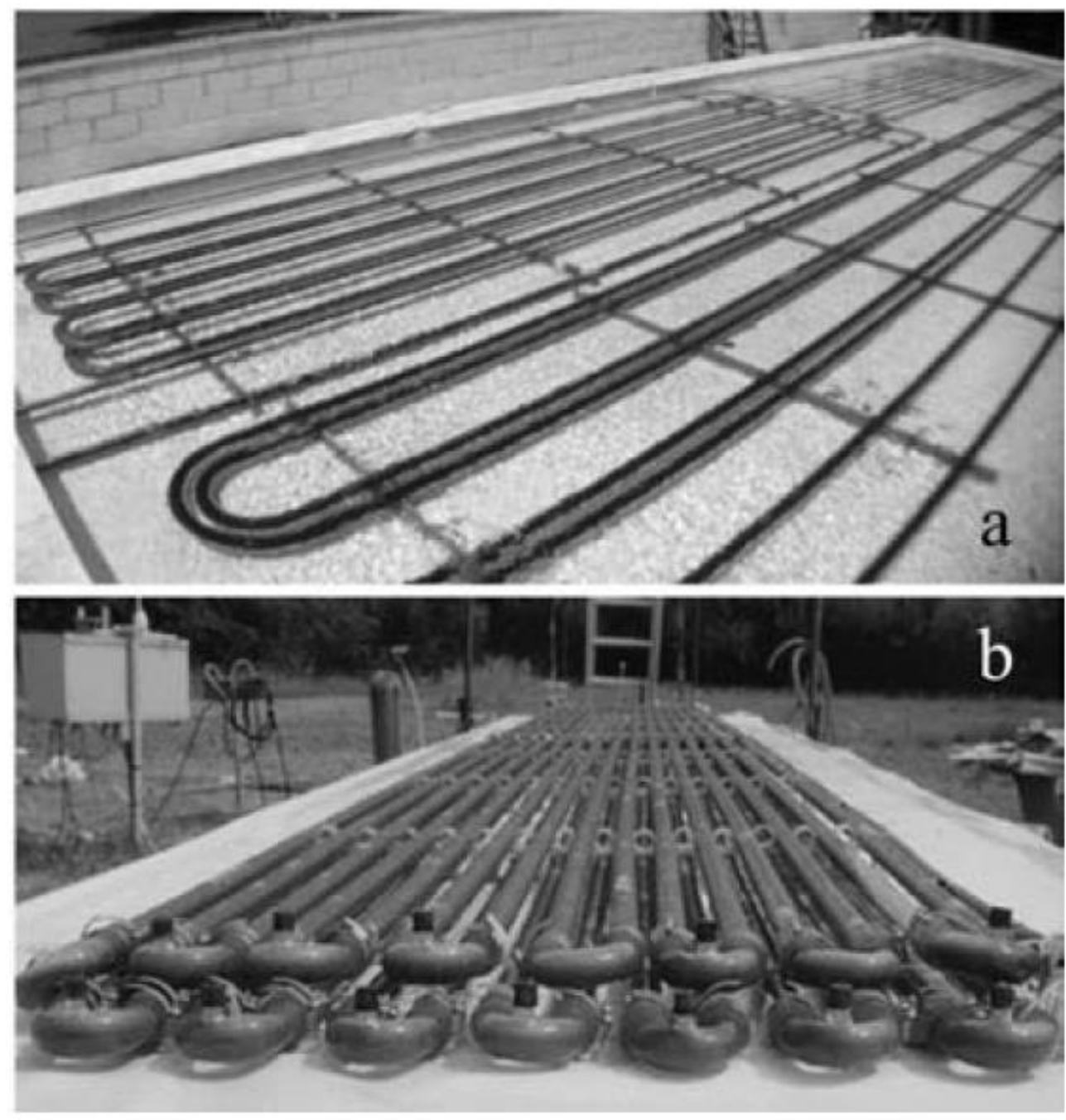

Figure 2.6. Serpentine systems of the 1990s (Tredici, 2004) 


\subsubsection{Manifold PBRs}

In a manifold PBR, a series of transparent tubes placed side-by-side at a slight angle to the horizontal (normally 4-6 $6^{\circ}$ are connected to two manifolds; one upper manifold and one lower manifold (Tredici, 2004). The upper manifold is for distribution of the culture in between the tubes, and for degassing, while the lower manifold is used for collection of the culture (Tredici, 2004). Tredici (2004) reported that manifold PBR allow for significant energy savings with respect to pumping the culture as compared to a serpentine system. Instead of moving the culture around U-bends, at each end the manifold reverses the direction of culture movement (Tredici, 2004).

Development of manifold PBRs is in most part credited to Tredici and co-workers in Italy (Tredici \& Chini Zittelli, 1998, Chini Zittelli et al., 1999), Richmond et al. (1993) in Israel, and Lee et al. (1995) in Singapore. Tredici and co-workers developed several 1,200-L near-horizontal manifold-type reactors (NHTR) to grow A. platensis, A. siamensis, Nannochloropsis sp., and P. tricornutum outdoors (Figure 2.7.a). Volumetric productivities of up to $1.3 \mathrm{~g} \mathrm{~L}^{-1} \mathrm{~d}^{-1}$ and areal productivities of more than $28 \mathrm{~g} \mathrm{~m}^{-2} \mathrm{~d}^{-1}$ were obtained with A. platensis (Tredici \& Chini Zittelli, 1998). The 133-L manifold reactor developed by Richmond et al. (1993) obtained average volumetric productivities of 0.55 $\mathrm{g} \mathrm{L}^{-1} \mathrm{~d}^{-1}$ with A. platensis, and A. siamensis (Figure 2.7.b). The 300-L manifold reactor created by Lee et al. (1995) obtained an average areal productivity of $72 \mathrm{~g} \mathrm{~m}^{-2} \mathrm{~d}^{-1}$. Other advantages of this type of reactor are the following: low shear stress, reduced biofouling and simplicity of operation (Tredici, 2004). However, tubes are limited to $40 \mathrm{~m}$ in length to prevent accumulation of photosynthetically-generated oxygen to toxic concentrations (Tredici, 2004). 


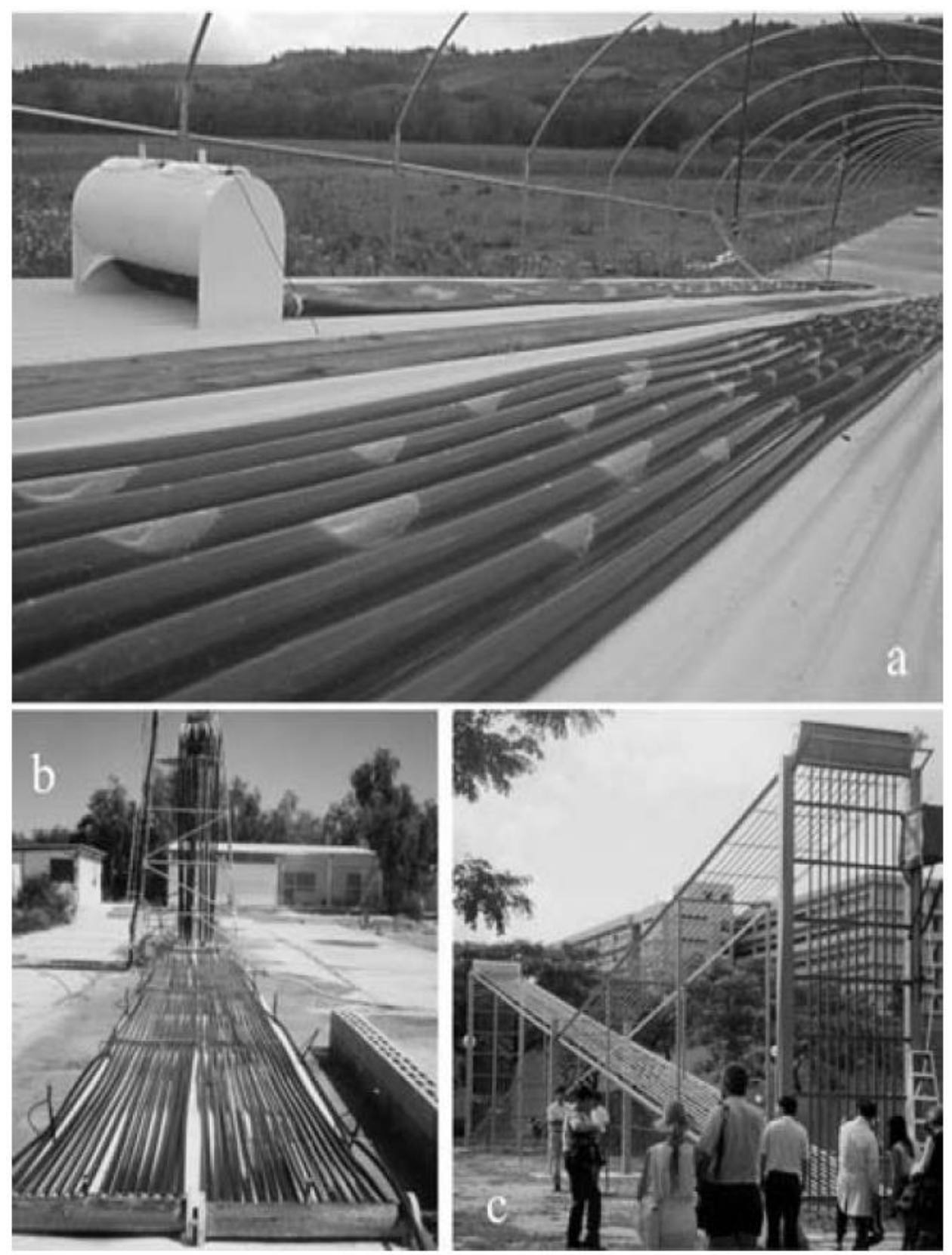

Figure 2.7. Manifold PBRs (Tredici, 2004)

\subsubsection{Helical PBRs}

A helical PBR consists of generally flexible transparent tubing (of very small diameter) wound around an upright structure (Figure 2.8). The design was first used by Davis et al. (1953) to grow Chlorella, and further development was done by Setlik et al. (1967), 
Krüger and Ellof (1981), Robinson and Morrison (1987), and Tredici and Chini Zittelli (1998). Setlik et al. (1967) and Krüger and Ellof (1981) came up with a flattened version made of glass tubes. Robinson and Morrison (1987) patented a helical PBR called biocoil, consisting of polyethylene tubing or PVC tubing wound around a cylindrical support. Tredici and Chini Zittelli (1998) devised and operated a 120-L helical PBR consisting of transparent PVC tubes wound around a vertical column with an inclination of $2^{\circ}$ to the horizontal. This PBR was able to attain a mean volumetric productivity of 0.9 $\mathrm{g} \mathrm{L}^{-1} \mathrm{~d}^{-1}$ with A. platensis (Tredici, 2004). This type of PBR offers even flow, shorter tube lengths, and minimum oxygen build-up and biofouling due to air being injected at each tube through a gas manifold for circulation and gas exchange (Tredici, 2004).

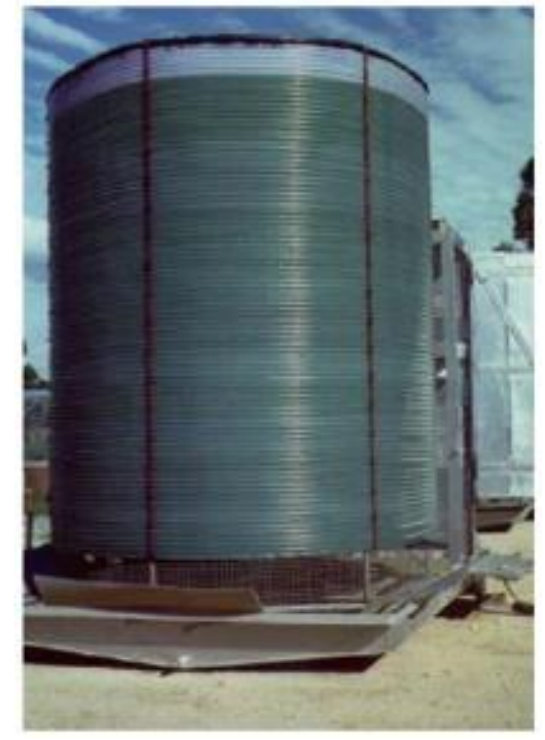

Figure 2.8. A 1000-L helical PBR at Murdoch University, Australia (Chisti, 2007)

\subsubsection{Fence Arrangement PBRs with Manifolds}

Applied Photosynthetics Limited (APL) (Manchester, UK) developed a PBR in 1998 known as bio-fence, which consists of an array of rigid transparent tubes racked together 
in banks and connected by manifolds in a fence-like structure (Figure 2.9). This PBR was specifically designed for either growing marine microalgae species used in the aquaculture business or for wastewater treatment with microalgae or photosynthetic bacteria (Tredici, 2004). The culture circulates in between a photo-stage and an airlift column, flow is provided by a pump or airlift, and $\mathrm{pH}$ is controlled by direct injection of $\mathrm{CO}_{2}$ in the photo-stage array (Tredici, 2004).

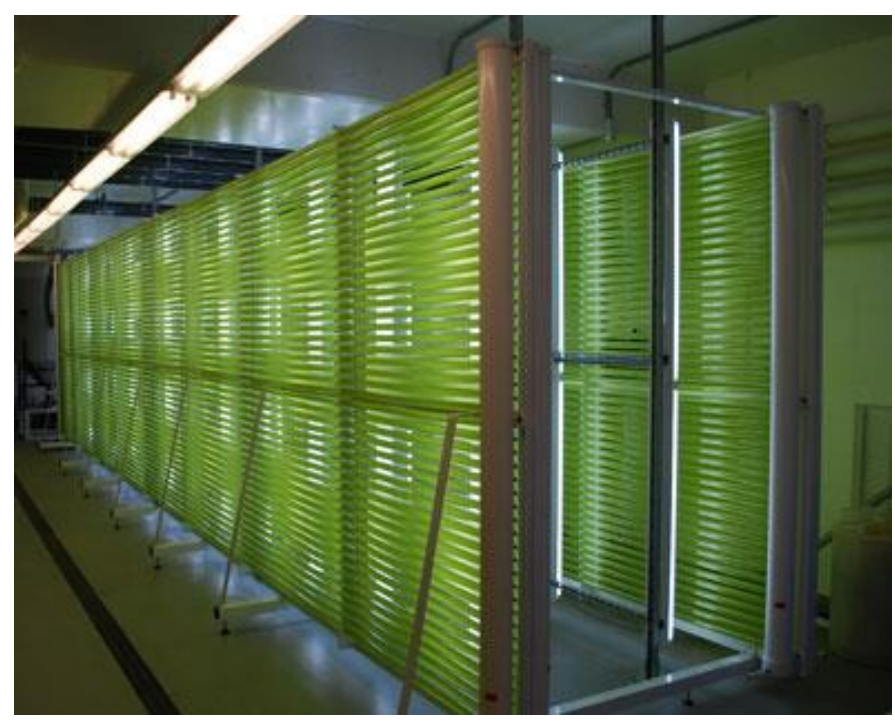

Figure 2.9. Biofence (National Research Council Canada, 2013)

\subsubsection{Flat Plate Photobioreactors}

Flat plate PBRs are made of transparent flat plates for the maximum utilization of light by the algae (Figure 2.10). The algae are cultivated in between these flat plates with the purpose of maximizing the surface-area-to-volume ratio and facilitating the measurement of irradiance at the surface of the culture (Molina, 2008). There is extensive published literature reporting high photosynthetic efficiencies with the use of these flat plate PBRs (Molina, 2008). However, this PBR design has been rarely used for mass cultivation of 
algae (Tredici, 2004). Flat plate PBRs can be subcategorized as flat alveolar panels and glass plates (Tredici, 2004).

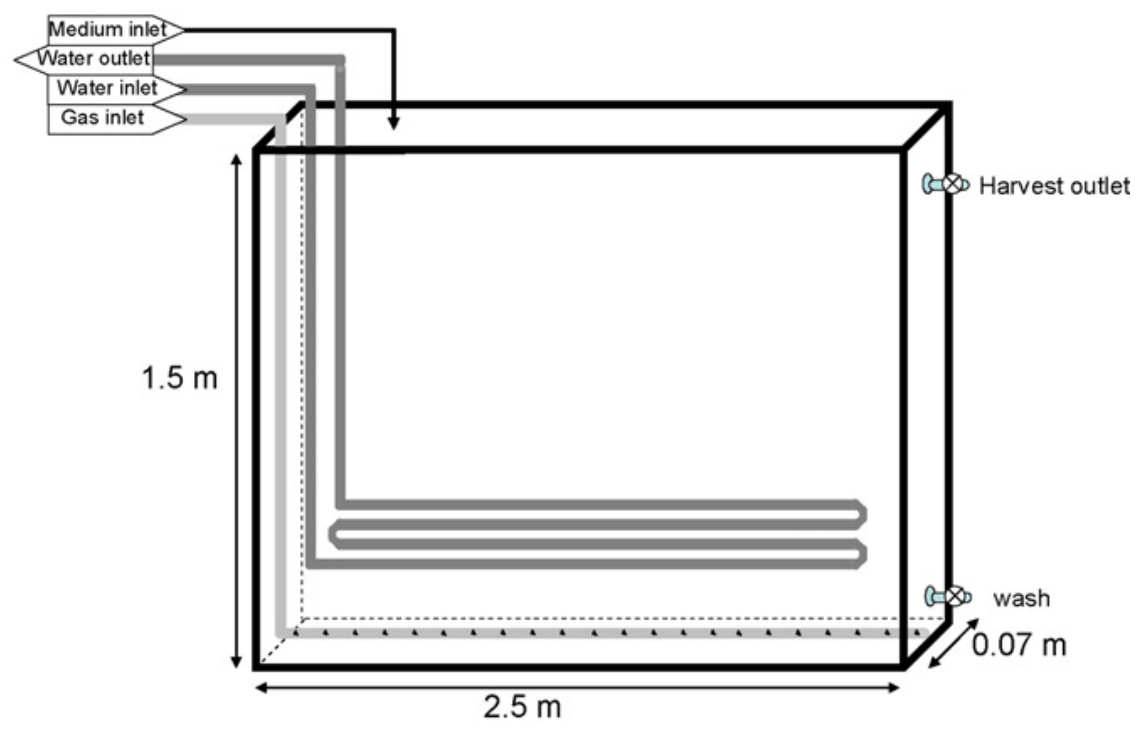

Figure 2.10. A flat plate photobioreactor (Molina, 2008)

The first design of a flat plate PBR was devised by Milner (1953) to grow Chlorella in a thin turbulent layer. Anderson \& Eakin (1985) made inclined flat plates to grow Porphyridium cruentum for polysaccharide production, and Samson \& LeDuy (1985) cultivated A. maxima in a 64-L vertical flat reactor. Samson \& LeDuy (1985) obtained volumetric productivities of $1.2 \mathrm{~g} \mathrm{~L}^{-1} \mathrm{~d}^{-1}$, corresponding to $60 \mathrm{~g} \mathrm{~m}^{-2} \mathrm{~d}^{-1}$ of illuminated surface productivity under artificial illumination with their reactor.

\subsubsection{Flat Alveolar Panels}

Based on earlier designs, Ramos de Ortega \& Roux (1986) in France, and Tredici and coworkers (Tredici et al., 1991) in Italy, further developed the flat-plate PBR concept. 
Ramos de Ortega \& Roux (1986) built a double-layer flat PBR with transparent PVC to grow Chlorella. The plates on this PBR were laid horizontally on the ground and had been partitioned to form narrow channels called alveoli (Tredici, 2004). The upper layer was used for algal growth and the lower layer for temperature control, with circulation provided by a pump (Tredici, 2004). An illuminated surface productivity of $24 \mathrm{~g} \mathrm{~m}^{-2} \mathrm{~d}^{-1}$ was attained with this PBR (Tredici, 2004). Tredici et al. (1991) introduced the idea of building vertically or highly inclined flat reactors with Plexiglas ${ }^{\circledR}$ and with the channels perpendicular to the ground. This later version, referred to as vertical alveolar panel (VAP), was extensively studied by this group for outdoor cultivation of microalgae and cyanobacteria (Tredici et al., 1991; Tredici \& Materassi, 1992; Tredici \& Chini Zittelli, 1997, 1998).

\subsubsection{Glass Plate PBRs}

In the mid-1990s Richmond and co-workers (Hu et al., 1996) devised and operated an inclined PBR made of a series of flat glass chambers connected in cascade and tilted at the optimal angle to maximize solar irradiation capture. These reactors were built to study the effect of diffused and reflected light on biomass productivity, and a maximum illuminated surface productivity of about $50 \mathrm{~g} \mathrm{~m}^{-2} \mathrm{~d}^{-1}$ was achieved (Tredici, 2004). Richmond (1999) and Richmond \& Zhang (2001) built a glass plate PBR to study the influence of light path on productivity of Nannochloropsis and A. platensis. By changing the thickness of the glass panels to change the light path, two important results were obtained: (1) the shorter the light path, the higher the volumetric productivity, and (2) optimal light path length changes with the algal species cultivated (Tredici, 2004). The 
maximum average illuminated surface productivity obtained was $12 \mathrm{~g} \mathrm{~m}^{-2} \mathrm{~d}^{-1}$,

corresponding to $0.24 \mathrm{mg} \mathrm{L}^{-1} \mathrm{~d}^{-1}$ of volumetric productivity (Tredici, 2004).

\subsubsection{Critical Evaluation of Flat Plate PBRs}

The studies carried out in Italy, Germany, and Israel on flat plate PBRs indicate that this type of PBR shows commercial promise. Elevated flat panels can be oriented and tilted at optimal angles for maximum utilization of light (Tredici, 2004). This feature is useful when it comes to seasonal variations in the angles of solar radiation and changes in latitude (Tredici, 2004). Also, utilization of $\mathrm{CO}_{2}$, mixing, degassing and temperature control are not normally problems with this concept (Tredici, 2004). However, alveolar panels can suffer from leaks from the glued connections, numerous internal walls promote significant wall growth and too many units are needed to scale up to commercial size (Tredici, 2004). Glass plates have some advantages over alveolar panels: they are easier to clean, are highly transparent, are resistant to weathering and can be tailored to the desired optical path (Tredici, 2004). But glass panels can be excessively heavy, fragile and costly (Tredici, 2004).

\subsubsection{Airlift Column PBRs}

Airlift column PBRs (Figure 2.11) are defined by a specific fluid circulation pattern where the circulation is created by a stream of gas that agitates the liquid phase, as opposed to a stirrer (Merchuk, 2003). In an airlift reactor the major patterns of fluid circulation are determined by the design of the reactor (Merchuk, 2003). Airlift reactors can be divided into three main types: (1) external loop vessels, in which circulation takes place through separate and distinct conduits; (2) baffled (or internal-loop) vessels, in 
which baffles placed strategically in a single vessel create the channels required for the circulation; and (3) columns with no baffles (Merchuk, 2003).

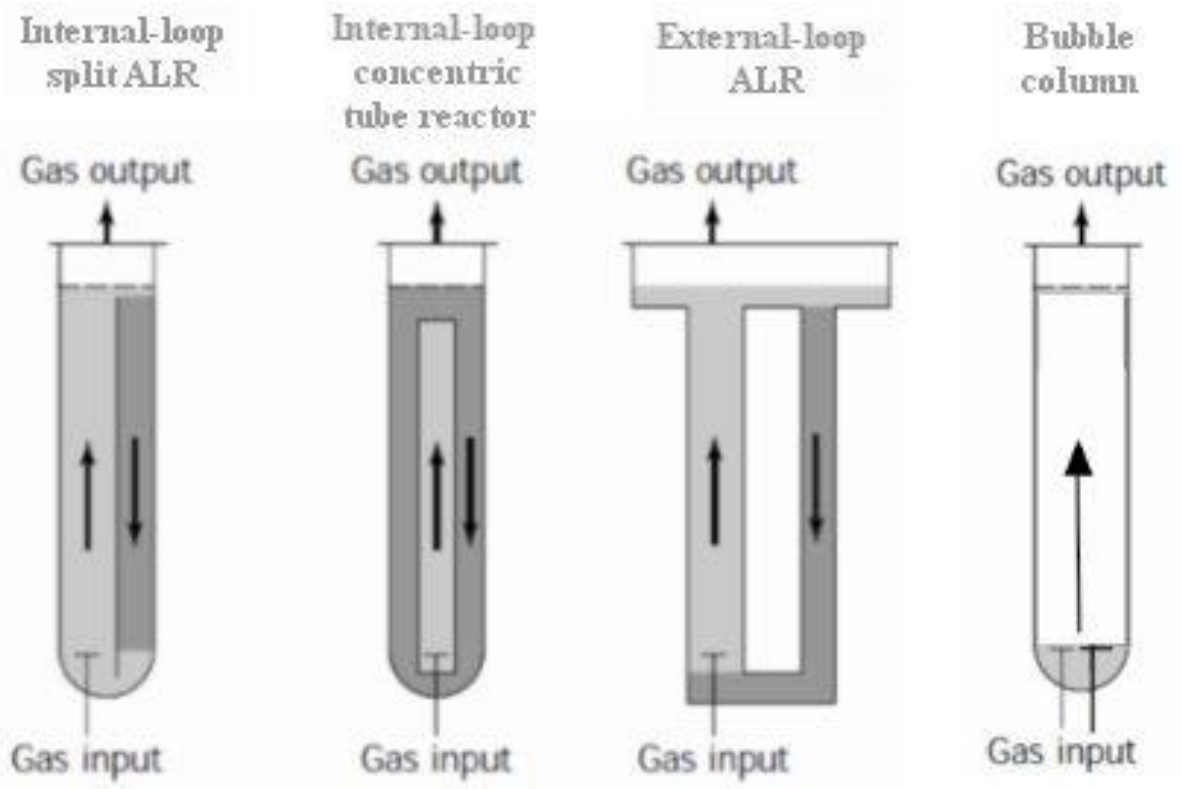

Figure 2.11. Different types of airlift column PBRs (Merchuk, 2003)

The airlift-column PBR concept was invented by Cook at Stanford University in California in the 1940s (Cook, 1950). These first units consisted of $1.8 \mathrm{~m}$-high, $10 \mathrm{~cm}$ diameter glass columns with a cone-shaped bottom. Maximum volumetric productivity indoors with Chlorella was $0.48 \mathrm{~g} \mathrm{~L}^{-1} \mathrm{~d}^{-1}$, while outdoors the volumetric productivity averaged $0.28 \mathrm{~g} \mathrm{~L}^{-1} \mathrm{~d}^{-1}$ with a maximum of $0.35 \mathrm{~g} \mathrm{~L}^{-1} \mathrm{~d}^{-1}$ (Tredici, 2004).

Further experimentation of airlift column PBRs was done by Jüttner (1982), Miyamoto et al. (1988), Hu \& Richmond (1994), Garcia Camacho et al. (1999), and Tredici and coworkers (2004). Jüttner (1982) built a 30-L glass-column reactor to grow Microcystis aeruginosa. Miyamoto et al. (1988) experimented with column reactors made of lowcost, mass-produced glass tubes used in the fluorescent glass industry and obtained 
volumetric productivities of about $0.6 \mathrm{~g} \mathrm{~L}^{-1} \mathrm{~d}^{-1}$ with Nostoc and Anacystis in outdoor cultivation. Using a similar system, Hu \& Richmond (1994) obtained volumetric productivities of $1.6 \mathrm{~g} \mathrm{~L}^{-1} \mathrm{~d}^{-1}$ with Isochrysis galbana cultivated outdoors. Garcia Camacho et al. (1999) developed a 12-L concentric-tube airlift reactor to cultivate $P$. tricornutum outdoors. And Tredici and co-workers (2004) developed a 135-L vertical annular reactor with internal illumination, made out of Plexiglas ${ }^{\circledR}$ to grow Nannochloropsis (Figure 2.12).

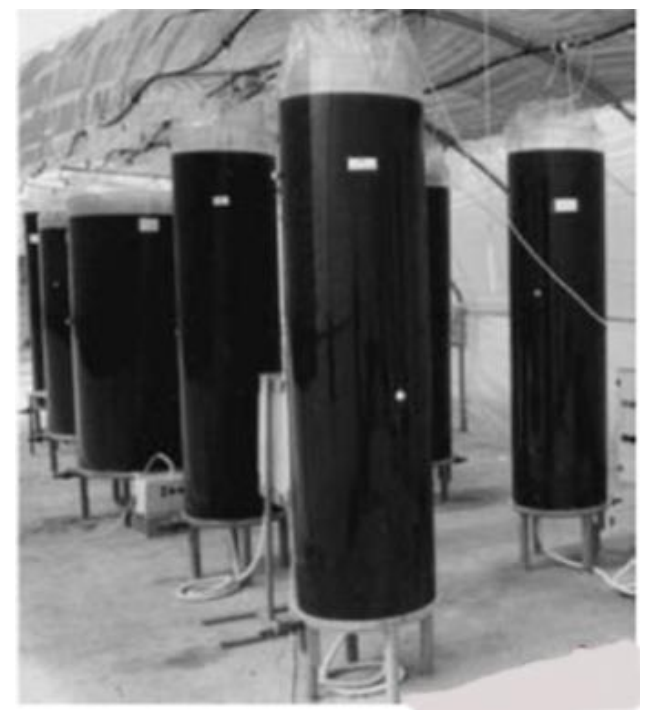

Figure 2.12. Airlift columns (Tredici, 2004)

An airlift PBR with tubes oriented in a vertical fashion and coupled with U-bends to connect adjacent tops and bottoms to form a flat loop was devised and patented by Cathcart (2011). This design is worth mentioning because it operates on the same fundamental principle as the PBR design studied in this thesis research, which is providing fluid circulation and gas exchange by airlift alone. The PBR design proposed by Cathcart (2011) consists of "down" columns and "up" columns (Figure 2.13). Flow and gas exchange is maintained by gas injection though diffusers located at the bottom of 
each column, and the gas is distributed through the columns by a gas manifold (Cathcart, 2011). The aeration rate in the "down" columns is lower than in the "up" columns to generate flow but still maintain good mixing, gas exchange and minimal biofouling in the entire reactor (Cathcart, 2011). The flow of gas in the alternating "up" columns is of sufficient force to move liquid upwards and provide enough momentum to cause downward flow to each adjacent "down" column (Cathcart, 2011). In this system no centrifugal pump is required (for appropriately sized modules) and the design addresses common issues encountered with tubular PBRs, which are excessive oxygen build-up in the tubes and shear stress to cells caused by a pump. However, no data about energy consumption improvements, flow regime, mixing, gas exchange or biomass productivity is provided in the patent. Nonetheless, this PBR design helped in the design of the prototype PBR studied in this thesis research.

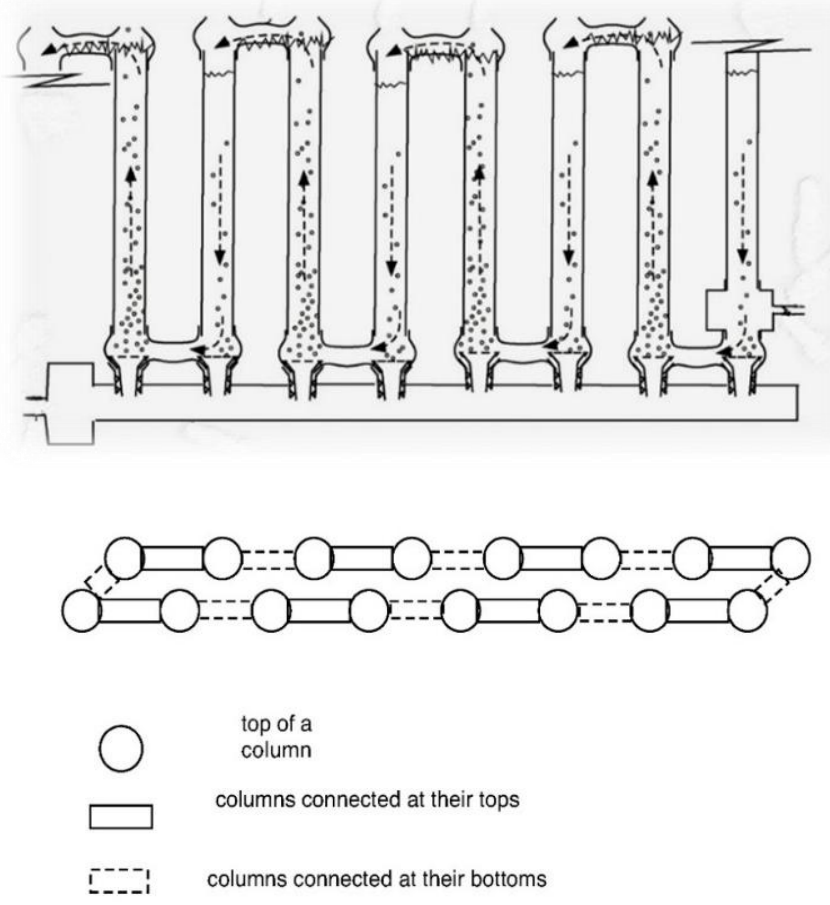

Figure 2.13. Schematic of the airlift PBR (Cathcart, 2011) 


\subsubsection{Vertical Sleeve PBRs}

Vertical sleeve PBRs use disposable tubes that are built by cutting a suitable length of transparent polyethylene tubing, heat-sealing one end, and suspending a series of bags or sleeves from a framework or mesh frame (Tredici, 2004). Several 2-m long sleeves have been used at the Institute of Applied Research in Beer-Sheva (Israel) (Figure 2.14) to grow Porphyridium and Dunaliella (Cohen \& Arad Malis, 1989). Such reactors can be used indoors and outdoors, have significantly better productivity than open ponds, and are easy and inexpensive to replace, but the concept suffers from relatively low $S / V$ ratio, biofouling and short life (due to the disposability of the tubes) (Tredici, 2004).

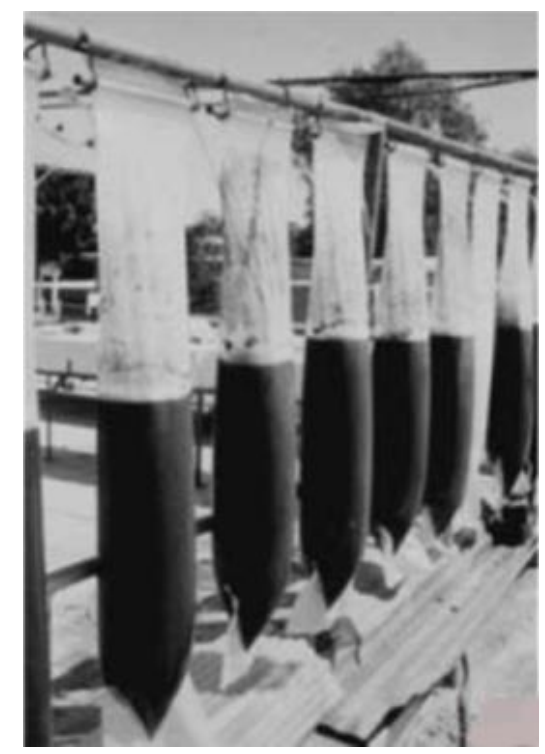

Figure 2.14. Vertical sleeves (Tredici, 2004)

\subsubsection{Fermenter-Type PBRs}

This type of PBR is best for heterotrophic cultivation of algae with appropriate carbon sources (Carvalho, 2006). This type of reactor has one major drawback: the S/V ratio is 
quite low, which in turns decreases the efficacy of harvesting sunlight if microalgae is to be cultured under phototrophic conditions (Carvalho, 2006). Pohl et al. (1988) devised a 250-L stainless steel fermenter-type PBR with internal illumination and a low-shear stress stirrer, but volumetric productivity was low $\left(30-50 \mathrm{mg} \mathrm{L}^{-1} \mathrm{~d}^{-1}\right)$. Thus, this type of reactor is only considered in the microalgae industry when microalgae is to be cultured under heterotrophic or photoheterotrophic conditions. The main advantages of this system are accurate control of processing parameters, the ability to maintain axenic cultures for long periods of time and a vast experience in the food and pharmaceutical industries (in terms of scale-up) for production of high-value metabolites (Carvalho, 2006).

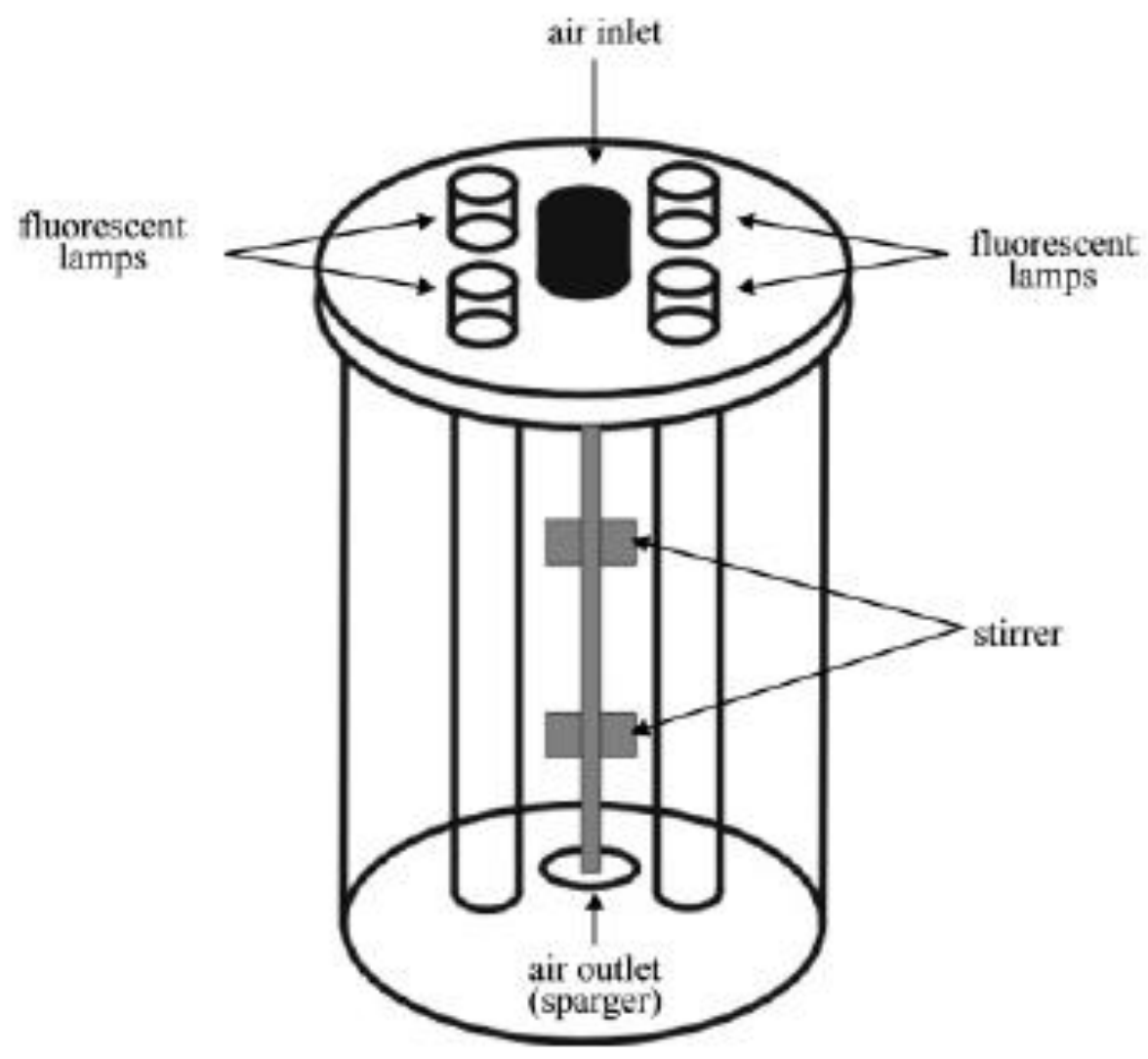

Figure 2.15. A fermenter-type PBR (Carvalho, 2006) 


\subsubsection{Attached-Growth PBRs}

Many species of microalgae and cyanobacteria tend to attach to surfaces when growing, and when cultured in a normal PBR, these microbes will tend to attach to the walls of the PBR and cause biofouling (Zittelli et al., 2013). Thus, specially designed PBRs have been designed to grow these kinds of microbes, because many of these species of microbes are attractive to the aquaculture and biofuel industry (Zittelli et al., 2013).

Attached-growth PBRs have the smallest water footprint and lowest harvesting costs than all other PBRs discussed above (Zittelli et al., 2013). However, the technology is limited to microbes that can be grown attached to a surface and, despite some claims, productivities are much lower than growing microalgae on suspended cultures (Zittelli et al., 2013). Thus, this technology would need more improvement.

\subsection{A Model Photobioreactor Located in Almeria (Spain)}

One of the largest microalgae pilot-plant PBRs in the world is located at the Experimental Station of Las Palmerillas, in Almeria (Spain) (Fernandez-Sevilla et al., 2010) and is a PBR of similar design to the prototype PBR studied in this research. The PBR units (Figure 2.16) are used to produce the lutein-rich, wild-type, mesophile microalgae strain Scenedesmus almeriensis (Sanchez et al., 2008; Acien et al., 2012). This facility consists of 10 tubular fence-type PBRs laid parallel to the ground (Dormido et al., 2014). Each PBR unit is made of a $400 \mathrm{~m}$-long serpentine tube of $0.09 \mathrm{~m}$ ID and about $22 \mathrm{~m}$ of straight pipe ending in common headers, with an airlift column $3.5 \mathrm{~m}$ high and $0.35 \mathrm{~m}$ ID, and has a working volume of 3,000 L (Dormido et al., 2014). The airlift columns are internal-loop split ALRs with a riser of $0.1 \mathrm{~m}$ ID and a downcomer of $0.25 \mathrm{~m}$ ID (Molina 
et al., 2006). The tube diameter and arrangement were optimized using the following constraints (Acien et al., 2012):

- Maximize culture volume per reactor

- Minimize yield losses caused by excessive light path

- Maximize interception of solar radiation

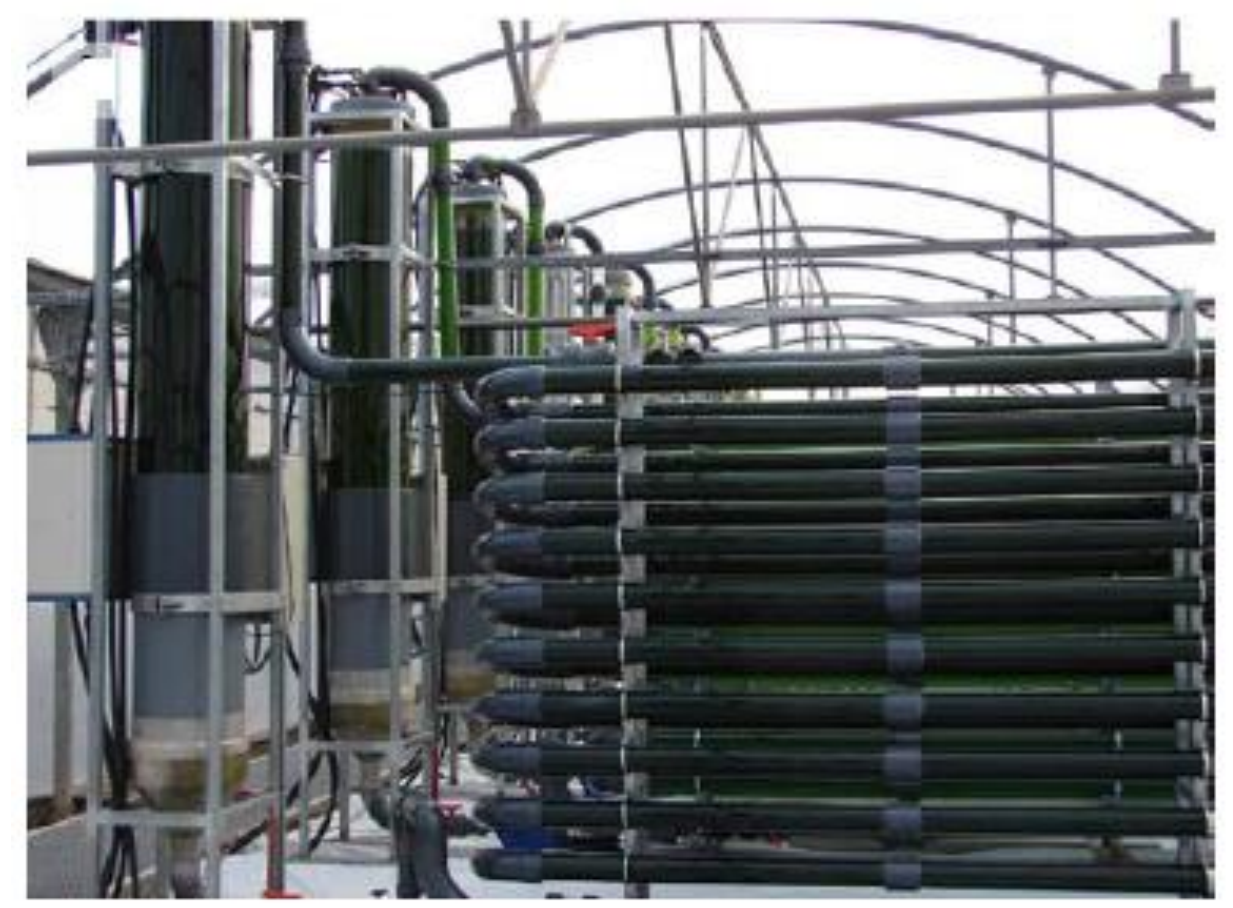

Figure 2.16. A 10-unit fence-type configuration totaling 30,000 L located in Almeria, Spain (Fernandez-Sevilla et al., 2010)

The tubular model (Figure 2.17) consists of two parts, which are the continuous tubular loop and an airlift column. The continuous tubular loop serves as the solar receiver and the airlift column is for heat exchange, degassing and nutrient addition (Acien et al., 2012). The culture is circulated with centrifugal pumps and airlift (Acien et al., 2012). Since most of the oxygen is generated in the solar receiver from photosynthesis, the 
culture is circulated with airlift in the airlift column to strip the oxygen with air $\left(\mathrm{O}_{2}\right.$ desorption) (Acien et al., 2012). In the solar receiver, a centrifugal pump is used to decrease the amount of air bubbles going into the solar receiver and prevent oxygen build up (Acien et al., 2012). To cool the PBR, water is pumped through a heat exchanger coil in the airlift column (Acien et al., 2012). Pure $\mathrm{CO}_{2}$ is supplied on demand as minute bubbles at the beginning of the tubular solar receiver for $\mathrm{pH}$ control purposes (Dormido et al., 2014).

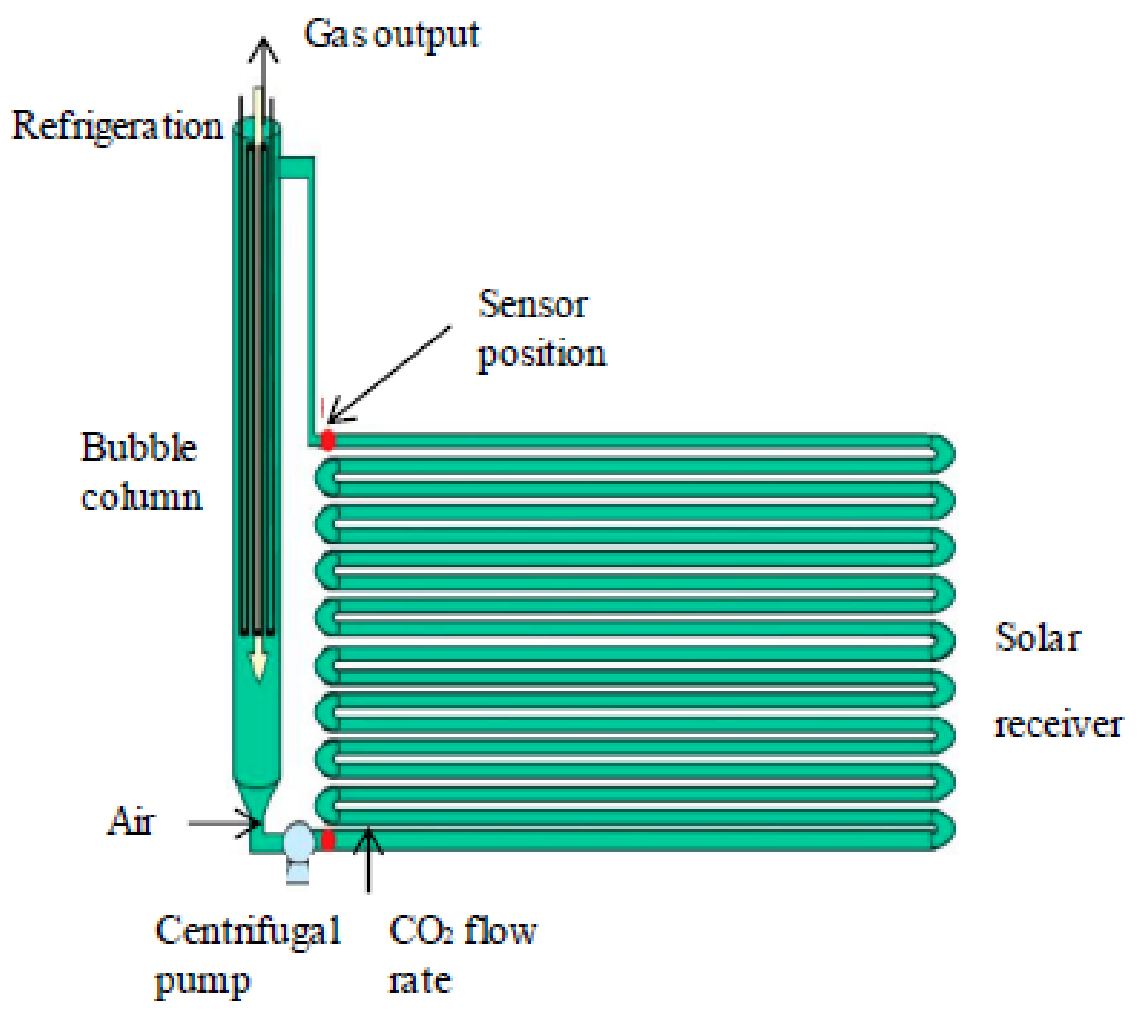

Figure 2.17. Schematic of the process (Dormido et al., 2014)

The complete system, including data acquisition and control software, were designed and built by the Department of Chemical Engineering at the University of Almeria (Almeria, Spain) (Dormido et al., 2014). The capital cost of the 10 -unit PBR is estimated at $\$ 8 / \mathrm{L}$ (in 
2014 \$) (Acien et al., 2010). Table 2.6 depicts the system specifications when growing Scenedesmus almeriensis. A 3,000-L unit in the Almeria PBR facility was chosen to compare to the Cal Poly PBR prototype studied herein due to similarities in structural design of both bioreactors.

Table 2.6. Specifications of the Almeria PBR facility when growing Scenedesmus almeriensis (Acien et al., 2010; Acien et al., 2012; Dormido et al., 2014)*

\begin{tabular}{lll}
\hline Parameter & Value & Unit \\
\hline S/V Ratio & 14.3 & $\mathrm{~m}^{-1}$ \\
Dilution rate & 0.34 & $\mathrm{~d}^{-1}$ \\
Mean maximum biomass concentration & 1.26 & $\mathrm{~g} \mathrm{~L}^{-1}$ \\
Operation time & 24 & $\mathrm{Hrs} \mathrm{d}^{-1}$ \\
Operation time & 300 & $\mathrm{~d} \mathrm{yr}^{-1}$ \\
Reactor Temperature & 35 & ${ }^{\circ}$ \\
Biomass production capacity & 3.83 & $\mathrm{Tons} \mathrm{yr}^{-1}$ \\
Efficiency of CO $\mathrm{CO}_{2}$ utilization & 75 & $\%$ \\
Air flow rate & 0.1 & $\mathrm{v}^{-} / \mathrm{min}^{-1}$ \\
Culture velocity & 0.9 & $\mathrm{~m} \mathrm{~s}^{-1}$ \\
Total unit volume & 3,000 & $\mathrm{~L}$ \\
Land area occupied by one unit & 40 & $\mathrm{~m}^{2}$ \\
Volume to land area ratio & 75 & $\mathrm{~L} \mathrm{~m}^{-2}$ \\
Photo-stage surface area of one unit & 110 & $\mathrm{~m}^{2}$ \\
Number of units & 10 & \\
Total plant volume & 30,000 & $\mathrm{~L}^{2}$ \\
Total culture land area & 400 & $\mathrm{~m}^{2}$ \\
Total reactor photo-stage surface area & 1100 & $\mathrm{~m}^{2}$ \\
Mean volumetric productivity (VP) & 0.42 & $\mathrm{~g} \mathrm{~L}^{-1} \mathrm{~d}^{-1}$ \\
Mean overall areal productivity (OAP) & 31.5 & $\mathrm{~g} \mathrm{~m}^{-2} \mathrm{~d}^{-1}$ \\
Mean illuminated surface productivity (ISP) & 11.5 & $\mathrm{~g} \mathrm{~m}^{-2} \mathrm{~d}^{-1}$ \\
Total plant power consumption & 448.2 & $\mathrm{kWh} \mathrm{day}^{-1}$ \\
Total specific plant power consumption & 15 & $\mathrm{kWh} \mathrm{m}^{-3}$ \\
Mean specific biomass productivity (SBP) & 7.81 & $\mathrm{mg} \mathrm{kJ}^{-1}$ \\
\hline *Note: All data in this table are over a period of one year, from January through December. \\
\end{tabular}

Table 2.7 shows power consumption of the main equipment used in the Almeria facility according to its power rating and time of use. Table 2.8 focuses on air blower and centrifugal pump power consumption for a single 3,000-L PBR module. The facility is equipped with all the necessary ancillaries for the automatic preparation of culture medium from fertilizers and fresh water (Acien et al., 2012). The main reason for using a 
centrifugal pump in tubular PBR designs of the type being studied is to decrease the amount of air bubbles going into the tubular solar receiver and to prevent dissolved oxygen from reaching concentrations above air saturation in the tubes; which is in general toxic to microalgae. However, this comes at the cost of high electrical power consumption for running the pump. A centrifugal pump also adds a significant amount of shear stress to cells, thus, these reactors are restricted to growing only microalgae types that are not sensitive to shear stress.

Table 2.7. Power consumption of the main equipment used in the Almeria facility (Acien et al., 2012)

\begin{tabular}{|c|c|c|c|c|c|}
\hline Equipment & $\begin{array}{l}\text { Power } \\
\text { (W) }\end{array}$ & $\begin{array}{l}\text { Operation } \\
\text { time } \\
\left(\mathrm{h} \mathrm{day}^{-1}\right)\end{array}$ & $\begin{array}{l}\text { Power } \\
\text { consumption } \\
\left(\mathrm{kWh} \mathrm{day}^{-1}\right)\end{array}$ & Units & $\begin{array}{l}\text { Power } \\
\text { consumption, } \\
\left(\mathrm{kWh} \text { day }^{-1}\right)\end{array}$ \\
\hline PBR centrifugal pump & 1000 & 24 & 24.0 & 10 & 240.0 \\
\hline Air blower & 4000 & 24 & 96.0 & 1 & 96.0 \\
\hline Decanter & 5500 & 6 & 33.0 & 1 & 33.0 \\
\hline $\begin{array}{l}\text { Culture medium } \\
\text { preparation unit }\end{array}$ & 1100 & 6 & 6.6 & 1 & 6.6 \\
\hline Ozone unit & 1100 & 6 & 6.6 & 1 & 6.6 \\
\hline Freeze dryer & 2200 & 24 & 52.8 & 1 & 52.8 \\
\hline \multirow[t]{2}{*}{ Control unit } & 550 & 24 & 13.2 & 1 & 13.2 \\
\hline & & & \multicolumn{2}{|c|}{ Total power consumption } & 448.2 \\
\hline
\end{tabular}

Table 2.8. Power consumption of the air blower and centrifugal pump for a single 3,000-L PBR unit in the Almeria facility (Acien et al., 2012)

\begin{tabular}{lllll}
\hline Equipment & $\begin{array}{l}\text { Power } \\
\text { (W) }\end{array}$ & $\begin{array}{l}\text { Operation time } \\
\left(\mathbf{h} \text { day }^{-1}\right)\end{array}$ & $\begin{array}{l}\text { Power } \\
\text { consumption } \\
\left(\mathbf{k W h ~ d a y ~}^{-1}\right)\end{array}$ & $\begin{array}{l}\text { Specific power } \\
\text { input } \\
\left(\mathbf{k W h ~ m}^{-\mathbf{3}} \mathbf{d a y}^{-\mathbf{1}} \text { ) }\right.\end{array}$ \\
\hline PBR centrifugal pump & 1000 & 24 & 24.0 & 8.0 \\
Air blower & 400 & 24 & 9.6 & 3.2 \\
& Total power consumption & 33.6 & 11.2 \\
\hline
\end{tabular}




\section{CHAPTER III: MATERIALS AND METHODS}

\subsection{Prototype Design Criteria}

The prototype was designed as a photobioreactor that has the advantages of an airlift column reactor and a tubular vertical reactor. Airlift column and vertical tubular systems were chosen due their superior capabilities when compared to other common designs based on reports by Carvalho, 2006; Ugwu et al., 2008; Das et al., 2011, and Oilgae, 2013. Unlike traditional tubular PBR designs and the PBR in the Almeria facility, the airlift-column units in this prototype are part of the photo-stage or solar receiver. The tubular stage of the prototype consists of vertical tubes that are connected by 180-degree fittings in a serpentine fashion; as opposed to the Almeria PBR which has its tubes positioned parallel to the ground. The Almeria PBR uses centrifugal pumps to circulate the culture around the serpentine tubing and an air blower for gas exchange in the airlift columns. The prototype PBR achieves circulation and gas exchange with just an air blower. Flow is induced by airlift gas in the columns and is of sufficient force and volume as to move media through the serpentine tubing. Bubbles perform both systemwide circulation and gas exchange. Although the bubbles in the tubes may not have the same degree of mixing effect as the bubbles in the column, there is still sufficient mixing in the tubes. Figure 3.1 depicts a 3D computer model of the Cal Poly prototype PBR, made with Google SketchUp 2013. The similarities between the Almeria PBR and the prototype PBR can be seen by comparing Figures 2.17 and 3.1. Both the prototype and the Almeria PBR are composed of an airlift stage and a tubular stage. 


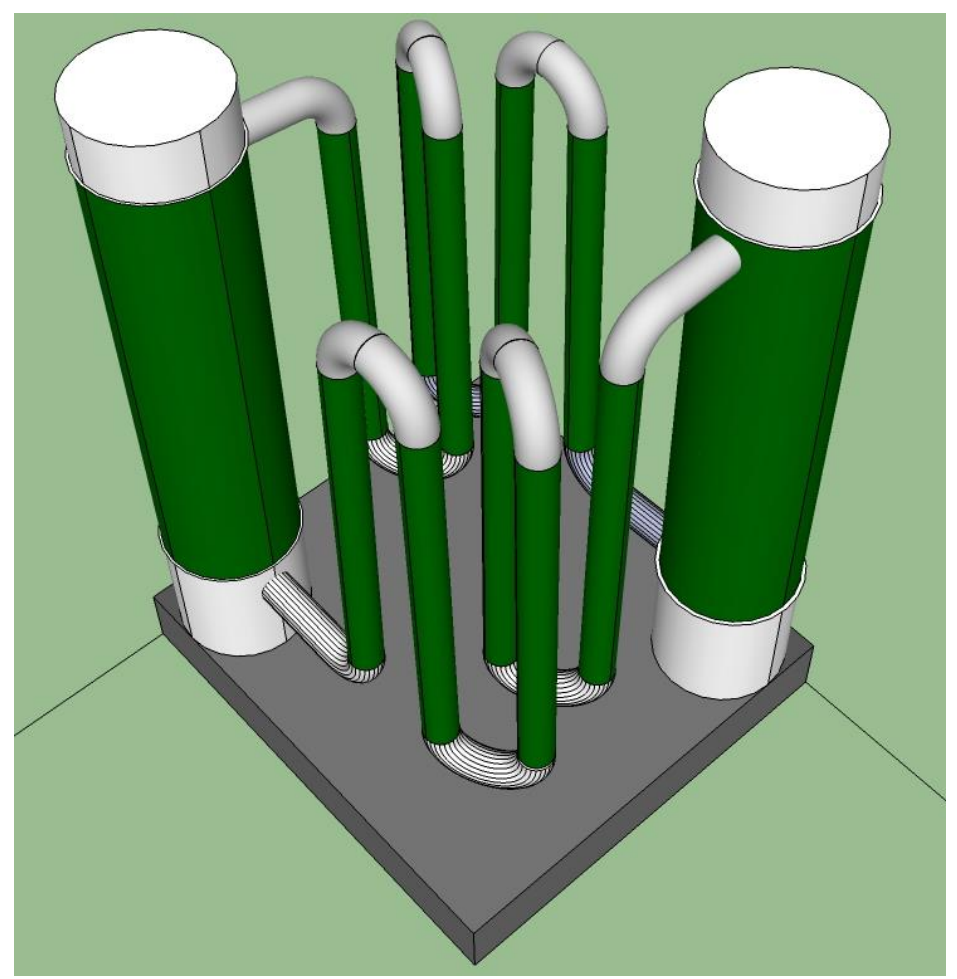

Figure 3.1. 3D model of the prototype PBR

The Cal Poly prototype design also takes into account ease of transportation and pilotscale operation. To be able to move the prototype to a new location (whether indoors or outdoors), it was constructed so as to allow loading the PBR into a standard pick-up truck with bed dimensions $8 \mathrm{ft}$ long $\mathrm{x} 4 \mathrm{ft}$ wide and into a standard elevator with dimensions 8 $\mathrm{ft}$ long x $6 \mathrm{ft}$ wide $\times 7 \mathrm{ft}$ tall and door dimensions $4 \mathrm{ft}$ wide $\mathrm{x} 6 \mathrm{ft}$ tall. The prototype can be easily moved with a pallet jack or a fork lift and disassembled for easy clean up, transportation and replacement of damaged parts. To be able to perform pilot-scale operations it was designed with a working volume of $235 \mathrm{~L}$.

The following is a summary of specific criteria that were used for the design of the Cal Poly prototype PBR: 
- Ease of transportation

- Pilot scale size (working volume $=235 \mathrm{~L}$, illuminated surface area of reactor photo-stage $=4.89 \mathrm{~m}^{2}$, total land area $=1.16 \mathrm{~m}^{2}$ )

- High volume-to-land-area ratio (203 $\mathrm{L} \mathrm{m}^{-2}$ occupied land area)

- Maximize number of input and output ports for quick drainage and fast oxygen degassing

- Continuous, steady, recirculating flow. Fast enough to prevent the algae from settling and provide sufficient oxygen transfer but not forceful enough at any point to present a significant portion of the culture to excessive shear forces and consume excessive electrical power

- Turbulent mixing effective at transporting cells between outer areas exposed to light and inner areas shaded by other cells

- High percentage of the flow path transparent and exposed to a light source

- Readily sterilizable, inexpensive and quick to take down, clean and restart.

- Provision for gas injection in an evenly distributed fashion

- Provision for out-gas extraction in an evenly distributed fashion

\subsection{Design Capacity and Geometry}

The Cal Poly prototype PBR consists of two airlift columns attached to $9 \mathrm{~m}$ of vertical serpentine tubing connected to the top and bottom by standard 90-degree PVC elbows in a U-bend fashion to each column to make a total working volume of $235 \mathrm{~L}$. The airlift columns are about $1.5 \mathrm{~m}$ tall and $30.5 \mathrm{~cm}$ ID, while the serpentine tubes are about $0.9 \mathrm{~m}$ tall and $7.6 \mathrm{~cm}$ ID. There are 5 vertical tubes for every airlift column, making a total of 
10 vertical tubes and 2 airlift columns. The serpentine tubing acts as the downcomer of the airlift columns. Unlike typical tubular PBR designs, the airlift columns are part of the photo-stage, this means that the whole reactor volume can receive solar radiation. In this reactor the culture loops around both airlift columns through the serpentine tubing (Figure 3.1).

Circulation of the culture was provided by rising bubbles produced by an air blower. The air blower was attached to disk diffusers that are located at the bottom of each airlift column. The airlift columns serve as media feed receivers. The PBR can be drained by gate valves located at the bottom of the serpentine tubing; the gate valves are all connected to a harvest pump by hoses. Sensors and probes were placed in the sampling ports located at the top of the serpentine tubing. Degassing took place in the empty headspace of each airlift column (where the photosynthetically generated oxygen was stripped out of the culture by the air diffusers) and through the sampling ports. $\mathrm{CO}_{2}$ was provided on demand for $\mathrm{pH}$ control purposes and for autotrophic cultivation.

The proposed PBR design also has a relatively high volume-to-land-area ratio. A 3,000 L PBR unit in the Almeria facility occupies a total land area (including empty space) of 40 $\mathrm{m}^{2}$, whereas the Cal Poly prototype PBR has a working volume of $235 \mathrm{~L}$ and requires a total land area of $1.16 \mathrm{~m}^{2}$. Thus, the volume-to-land-area ratio of a 3,000 $\mathrm{L}$ unit in the Almeria facility is $75 \mathrm{~L} \mathrm{~m}^{-2}$, whereas for the Cal Poly prototype PBR is $203 \mathrm{~L} \mathrm{~m}^{-2}$. Thus, the volume-to-land-area ratio of the Cal Poly prototype PBR is $271 \%$ that of a 3,000 L PBR unit in Almeria. Figure 3.1 depicts a 3D computer model of the prototype PBR, Figures 3.2, 3.4 and 3.5 show the dimensions of the prototype made with AutoCAD 2014, and Figure 3.3 depicts a photograph of the prototype in operation. 


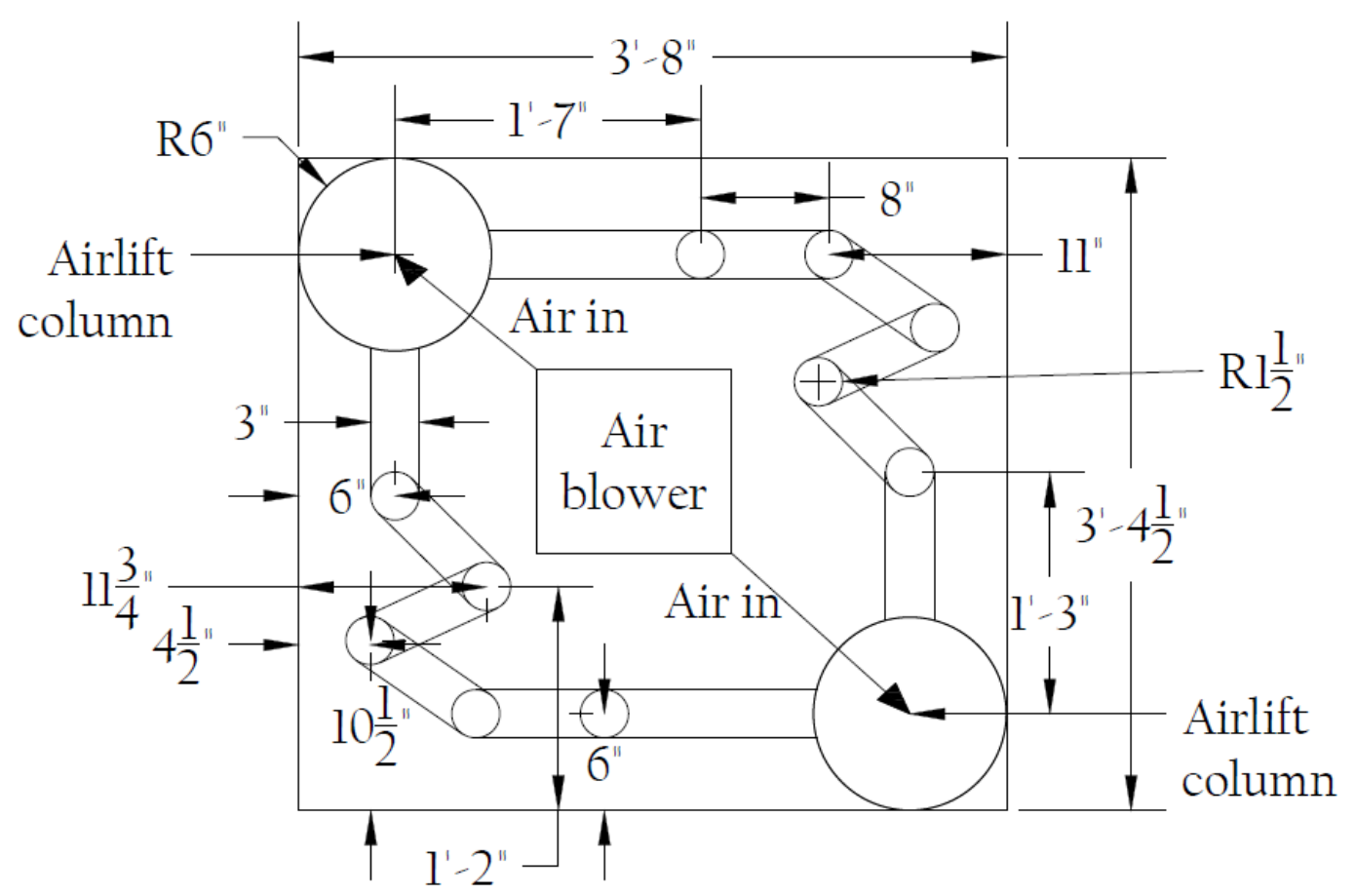

Figure 3.2. Schematic of overhead view of the prototype PBR

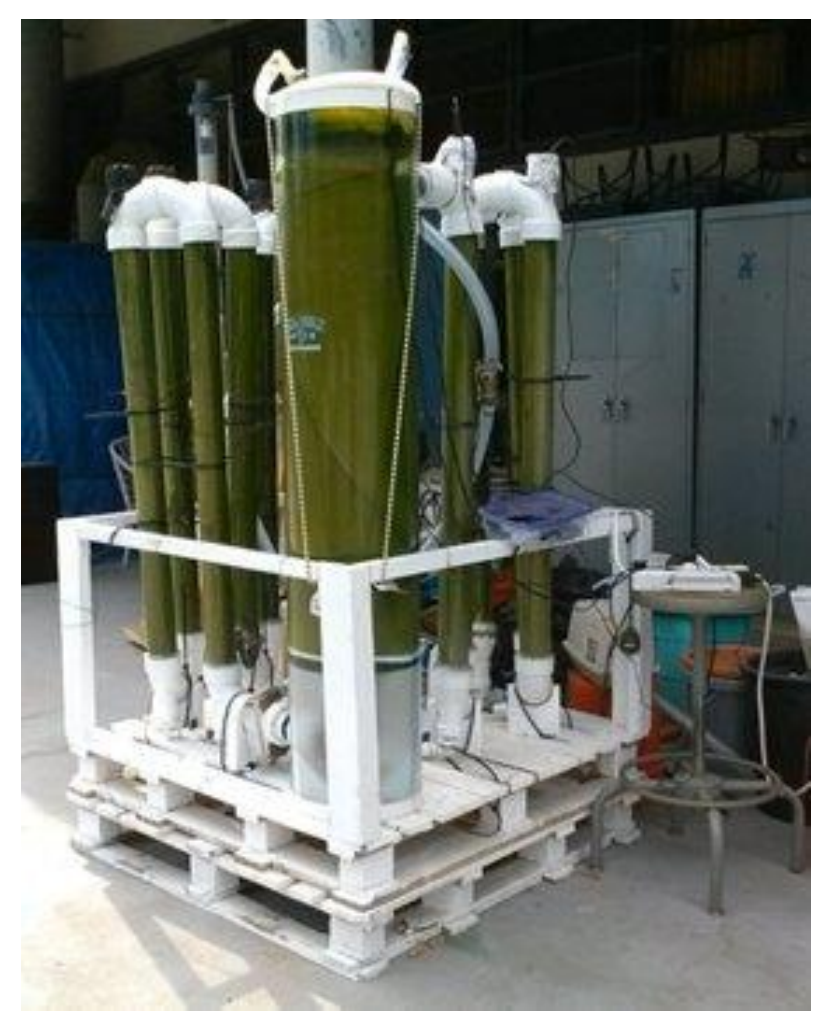

Figure 3.3. Side view of the prototype PBR 


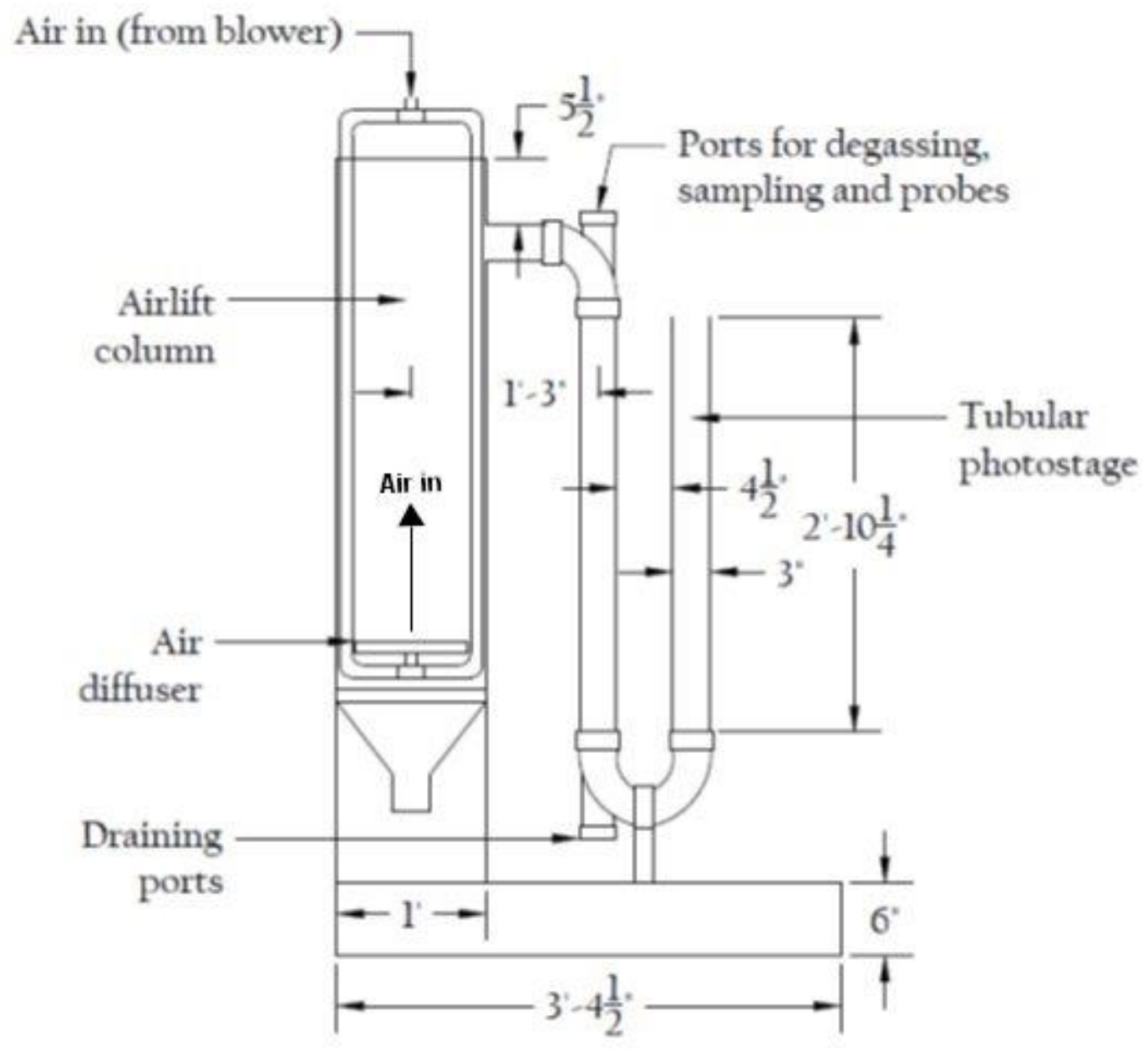

Figure 3.4. Schematic of side view of the prototype PBR showing culture exit port of the airlift column; culture flows from left to right (continues on next page) 


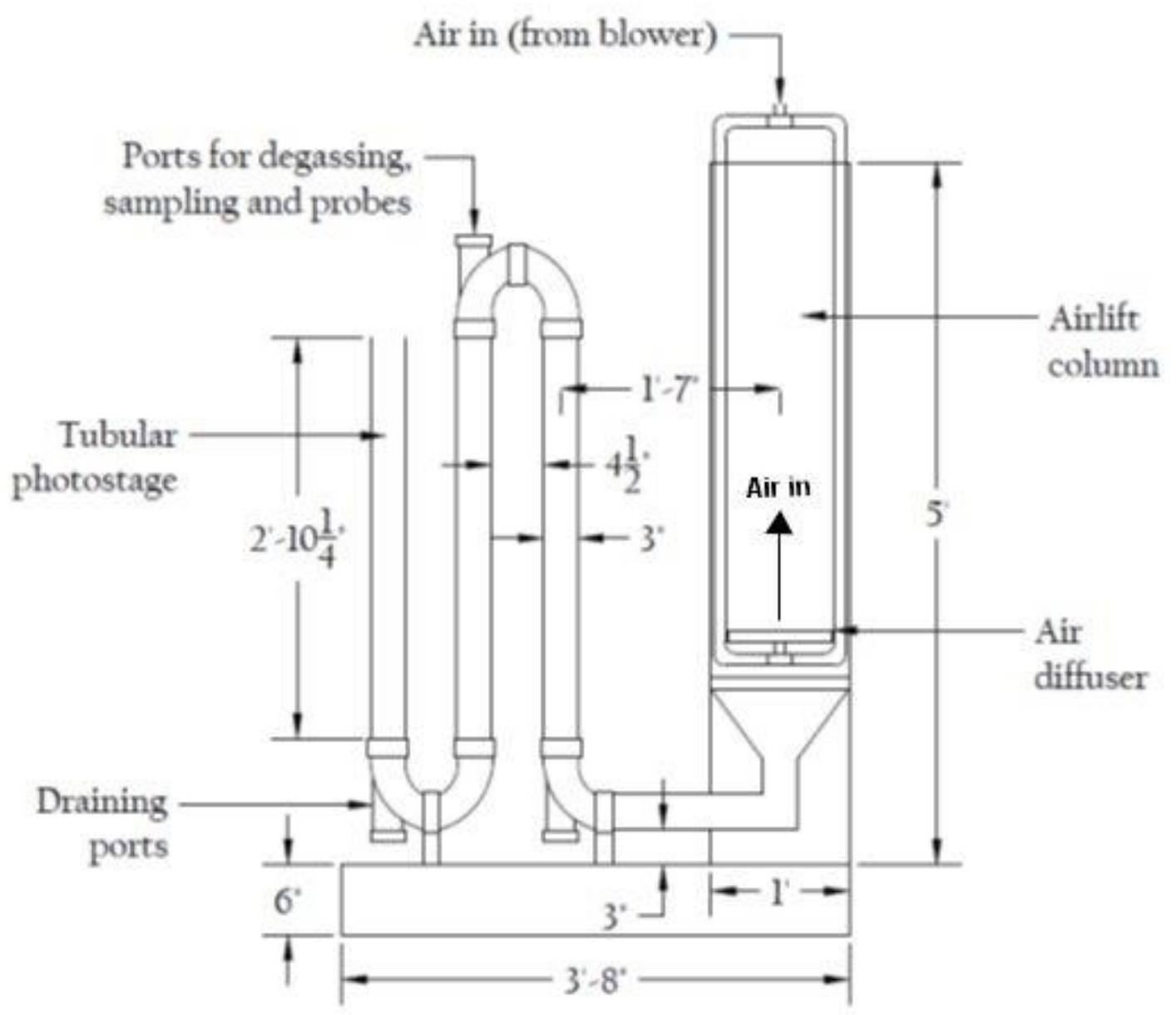

Figure 3.5. Schematic of side view of the prototype PBR showing culture entrance port of the airlift column; culture flows from left to right 


\subsection{Selection of Construction Materials}

Table 3.1 lists the major components for construction of the Cal Poly prototype PBR. The materials used are off-the-shelf materials, which are relatively inexpensive. It should be noted that the numbers shown in Table 3.2 are only indicative in nature since these costs can vary significantly by vendor and reactor volume. By adding up the total costs of all parts described in Table 3.1 (\$3628), and taking into account the working volume of the prototype $(235 \mathrm{~L})$, we can conclude that the capital cost of the Cal Poly design is about \$15/L (2014 \$). PBRs are expensive owing to their sophistication. Davis et al. (2011) reports that PBR costs are dominated by the price of the tubing system, and such a wide selection of tubing material currently available in the market creates challenges for predicting credible baseline capital costs for PBRs.

\section{Table 3.1. Construction materials for the prototype PBR}

\begin{tabular}{llll}
\hline Part & Quantity & Unit Price (\$) & Total Cost (\$) \\
\hline $\begin{array}{l}\text { 12" Diameter transparent plastic Conical } \\
\text { tanks (4' Height) }\end{array}$ & 2 & 397 & 794 \\
3" PVC Elbows & 20 & 12 & 240 \\
3" Diameter non-UV-resistant transparent & 40 feet & $1 /$ foot & 40 \\
PETG tubes & & & \\
1" Diameter transparent plastic hose & 50 feet & $2 /$ foot & 100 \\
Wooden base (3.5ft x 3.5ft) and frame & 1 & 60 & 60 \\
9" Bubble disk diffusers & 2 & 60 & 120 \\
PVC drain valves & 10 & 5 & 50 \\
Harvest pump & 1 & 80 & 80 \\
Air compressor & 1 & 592 & 592 \\
CO 2 Tank & 1 & 30 & 30 \\
pH regulator & 1 & 100 & 100 \\
Air flow regulator & 1 & 162 & 162 \\
Water heaters & 2 & 20 & 40 \\
CO 2 diffuser & 1 & 5 & 5 \\
pH sensor & 1 & 80 & 80 \\
Nitrate probe & 1 & 180 & 180 \\
Temperature probe & 1 & 30 & 30 \\
Dissolved oxygen probe & 1 & 210 & 210 \\
Light intensity sensor & 1 & 55 & 55 \\
Data loggers & 2 & 330 & 660 \\
\hline
\end{tabular}




\subsection{Design Components}

The prototype has the following components for operation (Figure 3.6):

- Air blower

- Air diffusers

- Air flow regulator

- Water heaters

- $\mathrm{CO}_{2}$ tank

- $\mathrm{CO}_{2}$ diffuser

- $\mathrm{pH}$ regulator

- $\mathrm{pH}$ sensor

- Temperature probe

- Nitrate probe

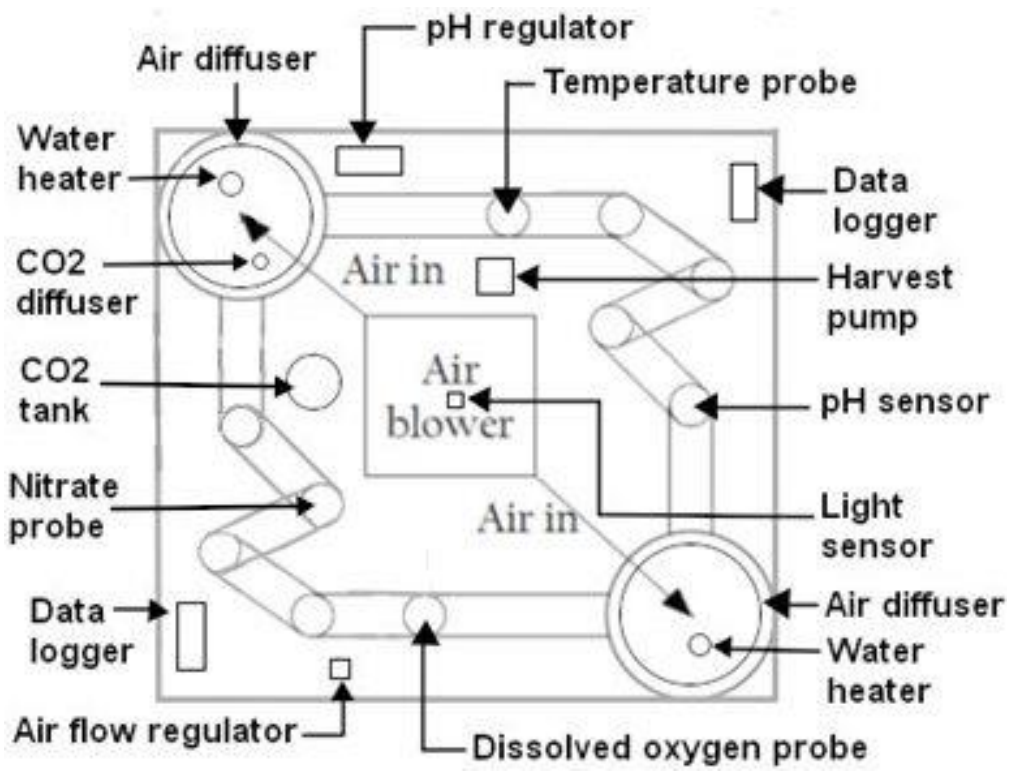

- Dissolved oxygen probe

Figure 3.6. Process control diagram

- Light intensity sensor

- Data logger

- Harvest pump

A Medo ${ }^{\circledR}$ LA-120A Air Blower (Figure 3.7) was used for culture circulation and gas exchange. The blower provided an air flow of $120 \mathrm{~L} / \mathrm{min}$ at its rated pressure of 0.18 bar, and has a power consumption of 118 Watts. 


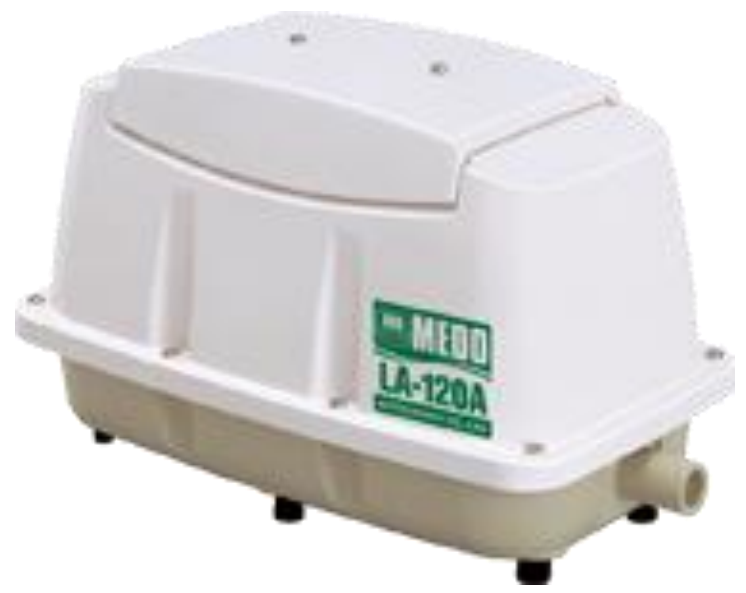

Figure 3.7. Air blower (Nitto-Kohki Co., 2014)

Two FlexAir ${ }^{\circledR}$ 9" Bubble Disk Diffusers (Figure 3.8) were connected to the air blower to diffuse air in the culture, thus, providing fluid circulation and enough turbulence for mixing the culture and gas exchange.

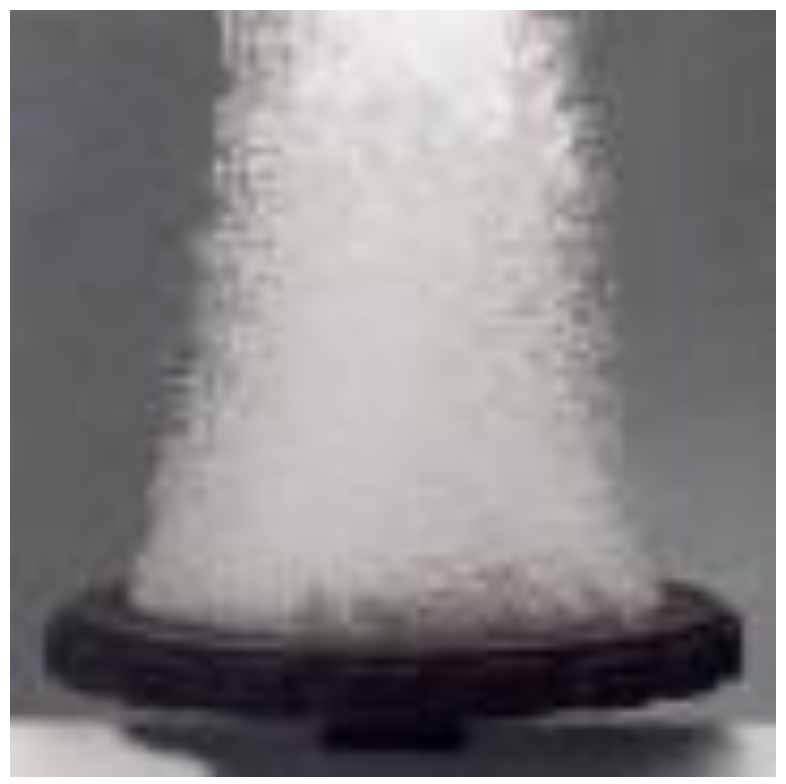

Figure 3.8. Disk diffuser (FlexAir, 2014) 
A Key Instruments ${ }^{\circledR}$ acrylic flowmeter (Figure 3.9) was connected to the air blower to regulate the air flow rate to the disk diffusers. This flowmeter uses a stainless steel ball float and has reading scales to measure the flow rate of air. The accuracy of its reading scales is $\pm 3 \%$.

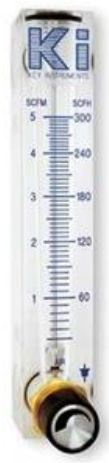

Figure 3.9. Air flow regulator (Key Instruments, 2014)

Two ViaAqua ${ }^{\circledR}$ Quartz Water Heaters (Figure 3.10) were used to keep the culture temperature fluctuation at a minimum and increase the average culture temperature. The temperature fluctuation was due to overnight temperature drops, since the culture was grown outdoors. These water heaters were rated at $300 \mathrm{~W}$ atts each.

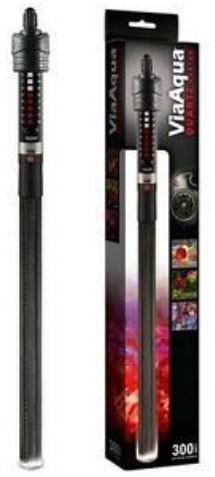

Figure 3.10. Water heater (ViaAqua, 2014) 
A 20-lb $\mathrm{CO}_{2}$ tank (Figure 3.11) was connected to the $\mathrm{PBR}$ to provide pure $\mathrm{CO}_{2}$ to the culture for autotrophic outdoor cultivation and $\mathrm{pH}$ regulation.

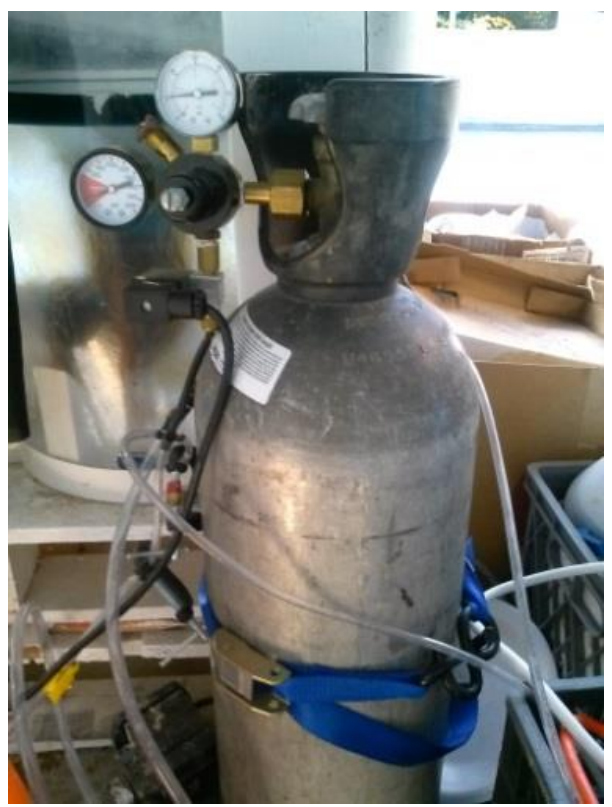

Figure 3.11. $\mathrm{CO}_{2}$ tank

A Viagrow ${ }^{\circledR}$ Micro Air Stone Diffuser (Figure 3.12) was connected to the $\mathrm{CO}_{2}$ tank through a plastic tubing to diffuse pure $\mathrm{CO}_{2}$ into one of the airlift columns of the PBR.

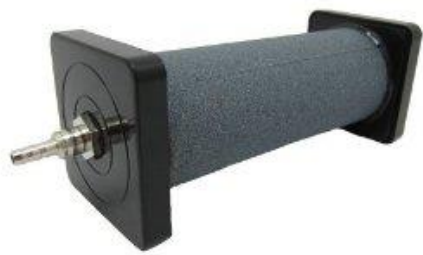

Figure 3.12. Air stone (Viagrow, 2014) 
A Milwaukee ${ }^{\circledR}$ SMS122 $\mathrm{pH}$ Meter (Figure 3.13) was used to regulate the $\mathrm{pH}$. This $\mathrm{pH}$ regulator was connected to a $\mathrm{CO}_{2}$ tank. The $\mathrm{pH}$ monitor allowed having a $\mathrm{pH}$ set point in the range of 5.5 to 9.5 . Once adjusted to a certain set point, the monitor would control the culture $\mathrm{pH}$ via injection of pure $\mathrm{CO}_{2}$. The set point used was 7.5 to grow C. vulgaris.

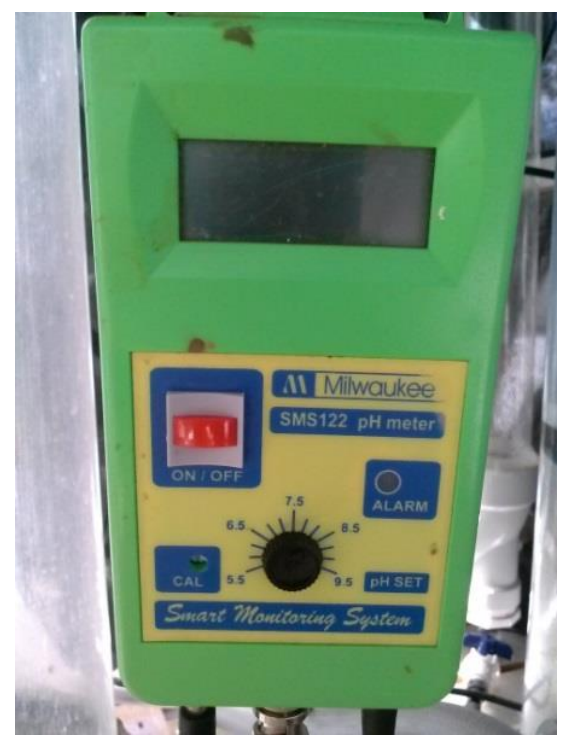

Figure 3.13. pH regulator (Milwaukee, 2014)

A Vernier ${ }^{\circledR} \mathrm{pH}$ Sensor (Figure 3.14) was used to measure the $\mathrm{pH}$ of the culture in the PBR.

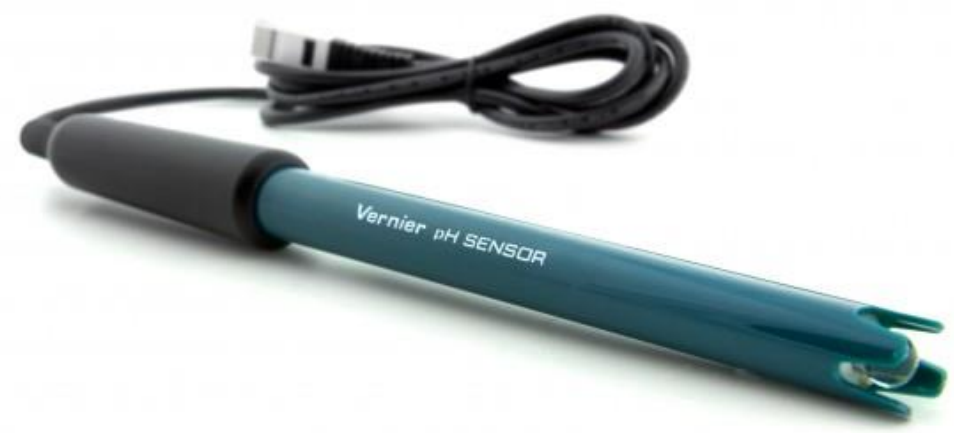

Figure 3.14. pH sensor (Vernier Software \& Technology, 2014) 
A Vernier ${ }^{\circledR}$ Stainless Steel Temperature Probe (Figure 3.15) was used to measure the temperature of the culture in the prototype PBR. This probe is a rugged, general-purpose laboratory temperature sensor.

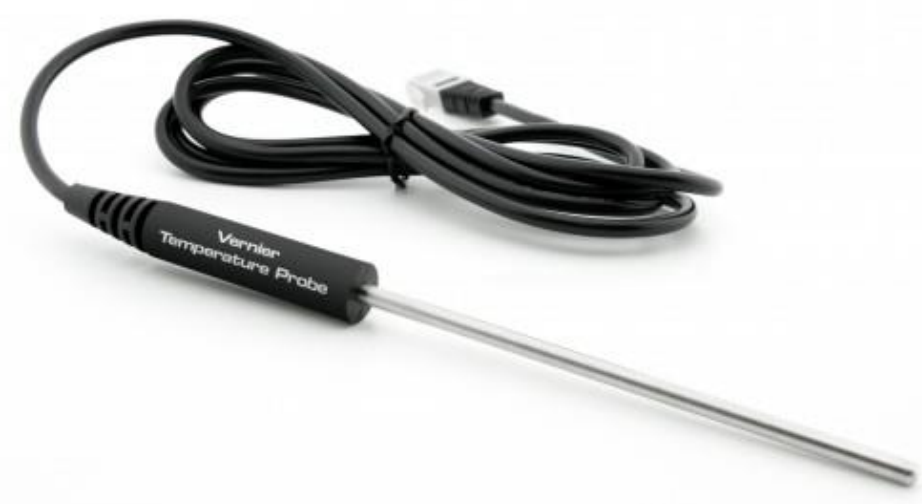

Figure 3.15. Temperature probe (Vernier Software \& Technology, 2014)

A Vernier ${ }^{\circledR}$ Nitrate Ion-Selective Electrode (Figure 3.16) was used to measure the concentration of $\mathrm{N}-\mathrm{NO}_{3}$ in the PBR samples.

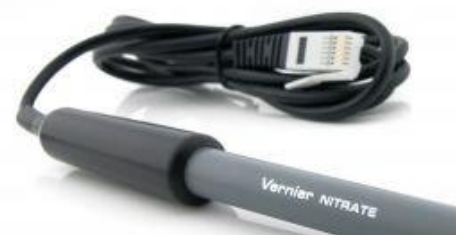

Figure 3.16. Nitrate ion-selective electrode (Vernier Software \& Technology, 2014) 
A Vernier ${ }^{\circledR}$ Dissolved Oxygen Probe (Figure 3.17) was used to measure dissolved oxygen concentration in the PBR.

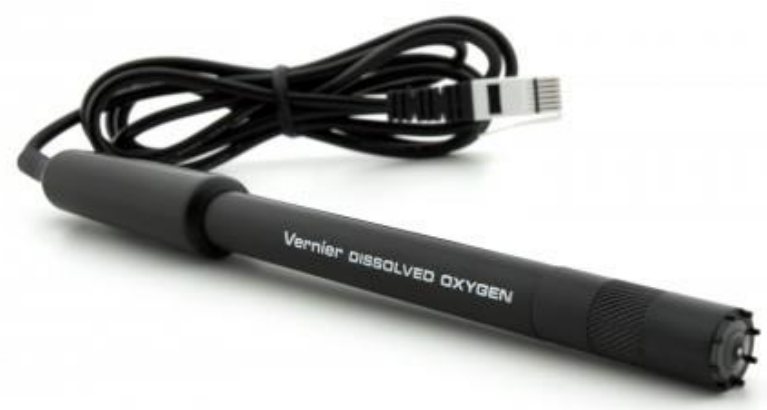

Figure 3.17. Dissolved oxygen probe (Vernier Software \& Technology, 2014)

A Vernier ${ }^{\circledR}$ Light Sensor (Figure 3.18) was used to measure the light intensity impinging in the reactor walls. This light sensor covers a range of 0 to 150,000 lux.

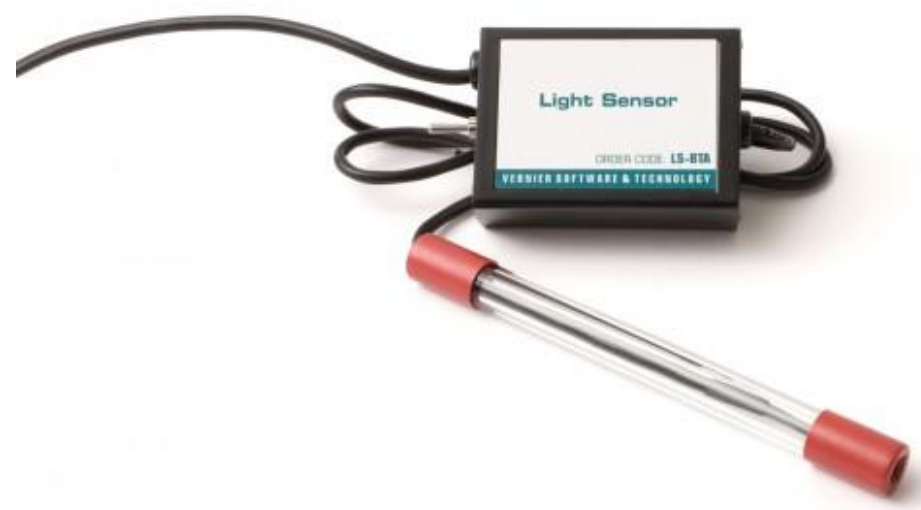

Figure 3.18. Light sensor (Vernier Software \& Technology, 2014) 
To collect $\mathrm{pH}$, temperature, light intensity, dissolved oxygen and nitrate concentration data points a Vernier ${ }^{\circledR}$ LabQuest $^{\circledR} 2$ Data Logger (Figure 3.19) was used. This data logger is a standalone interface used to collect sensor data with its built-in graphing and analysis application.

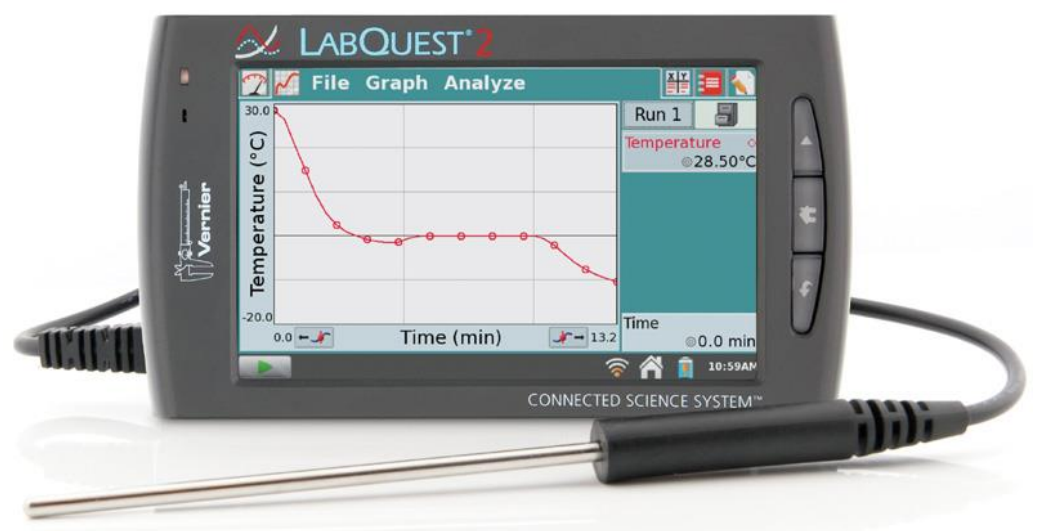

Figure 3.19. Data logger (Vernier Software \& Technology, 2014)

A Danner Manufacturing ${ }^{\circledR}$ Model 5 Magnetic Drive Pump (Figure 3.20) was used as the harvest pump. This pump has a capacity of $500 \mathrm{GPH}$.

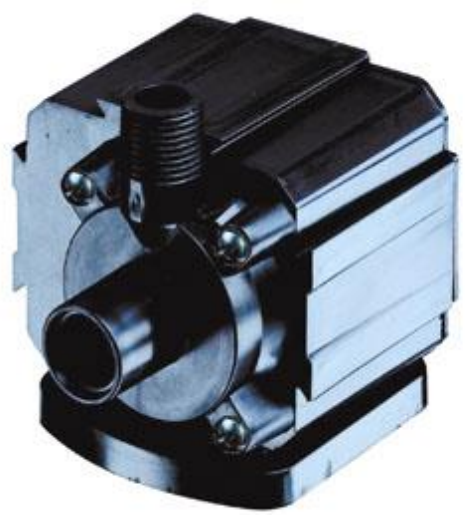

Figure 3.20. Harvest pump (Danner Manufacturing, 2014) 


\subsection{General Operation of the Prototype PBR}

The PBR was inoculated with Chlorella vulgaris, a wild-type mesophile microalgae strain, which was obtained from Carolina Biological Supply Company. The inoculum was first grown in 1-L of Bristol medium (Table 3.2) for about one week. The Chlorella inoculum culture reached a dark-green color in the flask after one week (Figure 3.21).

Table 3.2. Components and concentrations for $1 \mathrm{~L}$ of Bristol medium and $10 \mathrm{~mL}$ of stock solution of each component (UTEX, 2014)*

\begin{tabular}{llll}
\hline $\begin{array}{l}\text { Component } \\
\text { number }\end{array}$ & Component & $\begin{array}{l}\text { Stock Solution Conc. } \\
\left(\mathbf{g} / \mathbf{4 0 0} \mathbf{m L} \mathbf{~ d H}_{\mathbf{2}} \mathbf{O}\right)\end{array}$ & $\begin{array}{l}\text { Final Conc. in stock } \\
\text { solution }(\mathbf{m M})\end{array}$ \\
\hline 1 & $\mathrm{NaNO}_{3}$ & 10 & 2.94 \\
2 & $\mathrm{CaCl}_{2} \cdot 2 \mathrm{H}_{2} \mathrm{O}$ & 1 & 0.17 \\
3 & $\mathrm{MgSO}_{4} \cdot 7 \mathrm{H}_{2} \mathrm{O}$ & 3 & 0.3 \\
4 & $\mathrm{~K}_{2} \mathrm{HPO}_{4}$ & 3 & 0.43 \\
5 & $\mathrm{KH}_{2} \mathrm{PO}_{4}$ & 7 & 1.29 \\
6 & $\mathrm{NaCl}$ & 1 & 0.43
\end{tabular}

*Note: To approximately $900 \mathrm{~mL}$ of $\mathrm{dH}_{2} \mathrm{O}$ add $10 \mathrm{~mL}$ of each of the components in the order specified while stirring continuously. Bring total volume to $1 \mathrm{~L}$ with $\mathrm{dH}_{2} \mathrm{O}$. Cover and autoclave medium. Store at refrigerator temperature (UTEX, 2014).

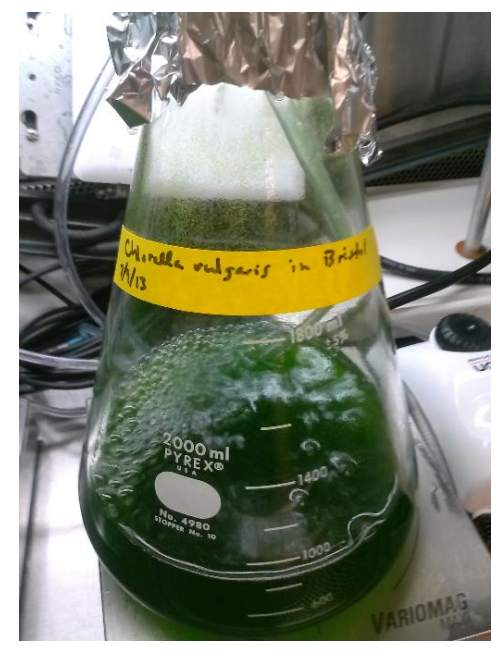

Figure 3.21. Culture of $C$. vulgaris in 1-L Bristol medium 
A week after letting the algae grow in the 1-L culture, the algae was diluted to $20 \mathrm{~L}$ and grown for another week before transferring to the PBR. The 20-L culture in the carboy depicted in Figure 3.22 also reached a dark-green color after one week.

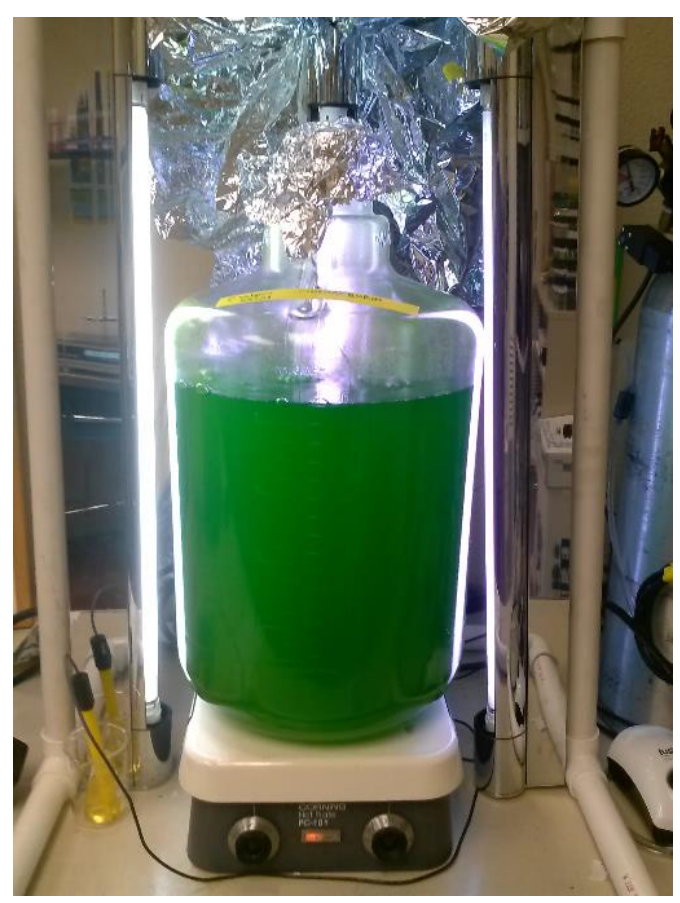

Figure 3.22. Culture of $C$. vulgaris in a 20-L carboy with Bristol medium

After letting C. vulgaris grow for another week in the 20-L culture it was then transferred to the PBR already sterilized and filled up with $235 \mathrm{~L}$ of dechlorinated tap water. The PBR was sterilized and dechlorinated by injecting ozone gas for 24 hours into the PBR through the gas diffusers located in the airlift columns. Before inoculating the PBR, 44 liters of the tap water were drained from the PBR to replace the drained volume with 20 liters of culture and 24 liters of freshly prepared Bristol 10x stock solution. 
The following steps were taken to operate the unit after preparation of the 20 -L inoculum:

1. Assemble and connect unit

2. Fill with tap water

3. Sterilize unit and dechlorinate tap water by means of ozone-gas injection

4. Add inoculum and medium

5. Initiate flow

6. Operate unit in partial shade until predetermined density is achieved

7. Operate unit under direct sunlight

8. Temperature controlled by leaving water heaters on overnight

9. Run system as the culture density increases.

10. $\mathrm{pH}$ is controlled by on-demand injection of $\mathrm{CO}_{2}$

11. Harvest by batch

12. Empty, disassemble and clean tubes, columns and U-couplers with a brush

\subsection{Method for Fluid Dynamics and Mass Transfer Characterization}

The influence of the aeration rate on the gas holdup capacity and overall mass transfer capacity of the reactor was studied. The purpose of this part of the study was to determine an overall mass transfer coefficient and optimum aeration rate. An optimum aeration rate would allow adequate oxygen transfer from the liquid phase to the gas phase while minimizing related power consumption.

The overall mass transfer coefficient, $K_{L} a$, was measured using the dynamic gassing-out method (Letzel et al., 1999). For $K_{L} a$ measurements the reactor was filled up with tap water. Dissolved oxygen was then removed from the reactor by sparging with nitrogen 
until the dissolved oxygen concentration reached about $10 \%$ of the dissolved oxygen concentration at saturation. Oxygen was then introduced in the reactor as compressed air using diffusers, and the dissolved oxygen concentration was monitored during reoxygenation of the liquid phase every second until saturation was reached. The overall gas holdup, $\varepsilon$, was quantified employing the volume expansion method. For overall gasholdup measuremnts the difference in height between the static liquid and the dispersed liquid upon aeration was measured as a function of aeration rate; as explained in the below subsections (Chisti, 1989).

Gas holdup and $K_{L} a$ measurements were repeated by increasing aeration rate by 0.05 $\mathrm{v} / \mathrm{v} / \mathrm{min}$ (volume air/volume culture/min) intervals; this unit is in common in the biotechnology industry. Re-oxygenation profiles from each experiment were analyzed separately. Low air flow rates were used ( 0.06 to $0.50 \mathrm{v} / \mathrm{v} / \mathrm{min}$ ) to minimize power consumption. Normally an aeration rate of $1 \mathrm{v} / \mathrm{v} / \mathrm{min}$ is used in bench-scale cultures (Sierra et al., 2008), but among the goals of this project is to find optimum conditions for large-scale cultures, which include low power consumption.

A tracer method was employed to determine liquid circulation velocity. A couple of drops of an $8 \mathrm{M}$ sodium hydroxide solution was poured instantaneously into one of the sampling ports and the $\mathrm{pH}$ was followed at two downstream locations by identical $\mathrm{pH}$ electrodes placed some distance apart in the tubular stage.

To better study the mixing pattern inside the reactor, aeration rate was studied as a function of mixing time. The mixing time, $t_{m}$, was determined by pulse response experiments in batch mode performed according to a procedure published by Sierra et al. 
(2008). It was determined as the time required to attain complete homogeneity after injection of a dye in the reactor. Mixing time is a direct indicator of mixing capacity of a reactor and is a useful parameter for comparing reactors in terms of characteristic mixing times (Sierra et al., 2008).

\subsection{Method for Evaluation of Biomass Productivity of the Prototype PBR}

Biomass productivity of the prototype PBR was evaluated by determining volumetric productivity (VP), illuminated surface productivity (ISP) and overall areal productivity (OAP) of the reactor under outdoor conditions using dry-weight (DW) measurements of the algae culture. The volumetric productivity was calculated using the following equation (Tredici, 2004):

$$
V P=\frac{\text { Total } \Delta g \text { DW biomass reactor }}{\text { L ractor } * \text { day }}
$$

the illuminated surface productivity was calculated with the following equation (Tredici, 2004):

$$
I S P=\frac{\text { Total } \Delta g D W \text { biomass in reactor }}{m^{2} \text { total illuminated surface area of reactor photostage } * \text { day }}
$$

and the overall areal productivity was also calculated using the following equation (Tredici, 2004):

$$
O A P=\frac{\text { Total } \Delta g D W \text { biomass in reactor }}{m^{2} \text { total land area occupied by reactor (including empty space) } * \text { day }}
$$


Samples of the culture in the reactor were taken approximately every 24 hours until biomass growth reached its stationary phase. The following techniques were used to determine the dry weight and cell count of the biomass in the samples. Calculation of VP, OAP and ISP for the prototype PBR was carried out using the data on biomass dry weight (Figure 4.18) of the exponential growth phase of Batch Run \#2 (from Day 8 to Day 12) and a method published by Shuler \& Kargi (2002) explained in detail in Section 4.4.

\subsubsection{Direct Microscopic Counting}

Cell concentration was determined by preparing wet mounts and counting the algal cells in a field of known area with an optical microscope and a hemacytometer counting grid (Fisher Scientific, U.S.). Wet mounts were prepared by pipetting $25 \mu \mathrm{L}$ of culture into each of the two microscope hemacytometer counting grids and placing a proper hemacytometer cover slip over the sample. The samples were viewed at 400X (Phase 2). Random fields were selected and all of the cells within the grid in the eyepiece were counted. Five fields in one grid were counted for good statistics and this was done for the two grids. The number of cells per $\mathrm{mL}$ in one grid were obtained with the following relationship because the grid volume was $250 \mu \mathrm{L}$ :

$$
\frac{\text { cells }}{m L}=(\text { Average number of cells in one grid }) x(250 \mu L) x\left(\frac{1 m L}{1000 \mu L}\right)
$$

The number of cells per $\mathrm{mL}$ of sample was obtained by taking the average of the two cell counts from each grid. 


\subsubsection{Dry Weight}

The total suspended solids (TSS) were determined daily. Samples were taken from the PBR at 10 PM each day and immediately tested for TSS. The testing for TSS was conducted according to Standard Methods 2540 D (APHA, 1995). The filters used for solids testing were 1.2-micron Fisher G4 glass fiber filters with 47-mm diameters. Mass measurements were made using a 5-decimal point electronic balance. Filters were first rinsed with DI water using a Millipore filter apparatus and then ashed in a muffle furnace at $525^{\circ} \mathrm{C}$ for $15 \mathrm{~min}$. The filters were then cooled in a dessicator and stored until needed in a $105^{\circ} \mathrm{C}$ oven. Just prior to analysis, filters were individually placed into aluminum foil trays and weighed. After weighing, the filter was placed on the Millipore filter apparatus and a known aliquot of sample (30-100 $\mathrm{mL})$ was passed through the filter. The filter was then removed using tweezers and placed back into its individual tray. Trays were then placed in the $105^{\circ} \mathrm{C}$ oven for at least 2 hours, but typically overnight. Trays were again weighed to obtain a TSS.

\subsection{Methods for Theoretical Scale-Up of the Prototype PBR}

One approach to theoretically scale up the Cal Poly prototype PBR to 3,000 L and be able to compare it to a 3,000-L PBR unit of the Almeria facility was to graph the power rating and air flow rate of various models of Medo ${ }^{\circledR}$ Air Blower brand (as seen in Figure 3.23). This figure depicts a linear relationship between power and air flow rate. This linear relationship was used as a method to estimate specific power input as a function of aeration rate in the Cal Poly prototype PBR and to scale up the size of the air blower needed to theoretically operate the prototype PBR at 3,000-L capacity. 


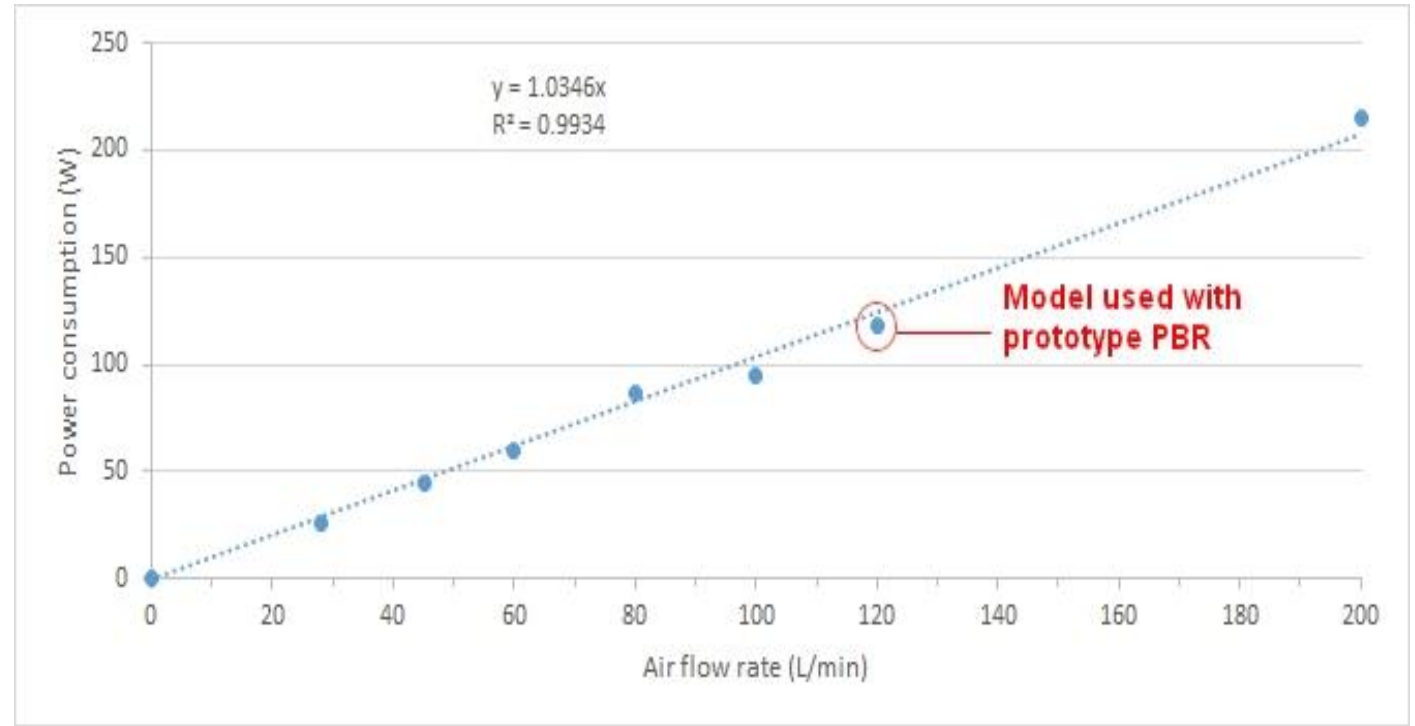

Figure 3.23. Available models of Medo $^{\circledR}$ air blower brand (Nitto-Kohki Co., 2014)

Another approach to calculate the required specific power input from an air blower to a projected 3,000-L Cal Poly PBR was to estimate the total head loss of the scaled-up PBR. To be able to determine the total head loss of the projected 3,000-L Cal Poly PBR, the height and diameter of the airlift columns were increased and the height of the vertical tubes were also increased while keeping the tube diameter constant and two airlift columns. In the prototype PBR, the airlift columns are about $1.5 \mathrm{~m}$ tall and $30.5 \mathrm{~cm} \mathrm{ID,}$ while the serpentine tubes are about $0.9 \mathrm{~m}$ tall and $7.6 \mathrm{~cm}$ ID. There are 5 vertical tubes for every airlift column, making a total of 10 vertical tubes and 2 airlift columns. The working volume of a single tube is about $4 \mathrm{~L}$ and the working volume of a single column is about $97.5 \mathrm{~L}$, thus, two columns and ten tubes add up to $235 \mathrm{~L}$. The dimensions of the projected 3,000-L Cal Poly PBR, using the approach described above, are the following: 
Column Dimensions

$H_{2}=4 m$ tall $, \quad D_{2}=0.4 m I D, \quad V_{2}=503 L$

$\frac{503 L}{\text { column }} * 2$ columns $=1,006 L$

Tube Dimensions

$H_{2}=4 \mathrm{~m}$ tall,$\quad D_{1}=D_{2}=0.076 \mathrm{mID} \rightarrow$ to be kept constant,$\quad V_{2}=18.1 \mathrm{~L}$

$3,000 L-1,006 L=1,994 L$

$\frac{1 \text { tube }}{18.1 \mathrm{~L}} * 1,994 \mathrm{~L}=110$ tubes

Serpentine tube length $=\frac{4 \mathrm{~m}}{\text { tube }} * 110$ tubes $=440 \mathrm{~m}$

Total photostage surface area $=10 \mathrm{~m}^{2}($ columns $)+105 \mathrm{~m}^{2}($ tubes $)=115 \mathrm{~m}^{2}$

\subsection{Method for Evaluation of Power Consumption of PBR in Almeria (Spain)}

A performance parameter often used to compare bioreactors is the specific power input; which is the power input per unit volume necessary for culture circulation and gas exchange in the reactor (Chisti, 1989). Each of the 3,000-L units of the Almeria PBR facility shares a single air blower connected to the bubble column to mix the culture and strip the photosynthetically-generated oxygen, but each module uses its own centrifugal pump to circulate the culture around the array of tubes. The following is an evaluation of 
the specific power input of the air blower and centrifugal pump for a 3,000-L unit of the Almeria PBR facility.

\subsubsection{Evaluating the Specific Power Input of the Air Blower}

To calculate the specific power input from the air blower to an airlift column Equation 3.5 was used. In pneumatically agitated reactors all the power supply originates from two sources: (i) the kinetic energy of the gas injected into the reactor, and (ii) isothermal expansion of the gas as it moves up in the riser; which is the predominant source of power (Chisti, 1989). The power input per unit volume due to operation, $P_{G} / V_{L}$, was calculated as a function of the density of the liquid, $\rho_{L}$, the gravitational acceleration, $g$, the superficial gas velocity on the riser, $U_{G r}$, cross-sectional area of the downcomer section, $A_{d}$, and cross-sectional area of the riser section, $A_{r}$, as follows (Chisti, 1989):

$$
\frac{P_{G}}{V_{L}}=\frac{\rho_{L} g U_{G r}}{1+\frac{A_{d}}{A_{r}}}
$$

Eq. 3.5 applies to airlift columns only and ignores the kinetic energy contribution of the specific power input since this contribution is $1.5 \%$ of the total power input according to Chisti (1989). Table 3.3 shows the specifications that were used with Equation 3.5 to calculate the specific power input. To calculate the specific power input from the air blower to an airlift column it was assumed that the algal culture has the same density of water at the specified temperature. The calculated specific power input with Eq. 3.5 was $5.5 \mathrm{kWh} \mathrm{m}^{-3}$. Acien et al. (2012) reported the specific power input from the air blower to a 3,000-L PBR module as $3.2 \mathrm{kWh} \mathrm{m}^{-3}$ (Table 2.8). Results of the calculation of the specific power input of the air blower are summarized in Table 4.5. 
Table 3.3. Almeria PBR specifications used to calculate the specific power input of the air blower (Molina et al., 2006; Acien et al., 2012; Dormido et al., 2014)

\begin{tabular}{lll}
\hline Parameter & Value & Unit \\
\hline Air flow rate & 80 & $\mathrm{~L} \mathrm{~min}^{-1}$ \\
Working volume & 3.0 & $\mathrm{~m}^{3}$ \\
Riser cross-sectional area & 0.0079 & $\mathrm{~m}^{2}$ \\
Downcomer cross-sectional area & 0.0491 & $\mathrm{~m}^{2}$ \\
Air velocity in the riser, $U_{G r}$ & 0.169 & $\mathrm{~m} / \mathrm{s}$ \\
Reactor temperature & 35 & ${ }^{\circ} \mathrm{C}$ \\
Water density at $35^{\circ} \mathrm{C}, \rho_{L}$ & 994 & $\mathrm{Kg} \mathrm{m}^{-3}$ \\
Gravitational acceleration constant, $g$ & 9.81 & $\mathrm{~m} \mathrm{~s}^{-2}$ \\
Operation time & 24 & $\mathrm{hrs} \mathrm{day}^{-1}$ \\
\hline
\end{tabular}

\subsubsection{Evaluating the Specific Power Input of the Centrifugal Pump}

To calculate the specific power input of the centrifugal pump, the specifications in Table 3.4 and assumptions in Table 3.5 were used. To calculate the specific power input of the centrifugal pump it was assumed that the algal culture has the same density and dynamic viscosity of water at the specified temperature.

Table 3.4. Almeria PBR specifications used to calculate the specific power input of the centrifugal pump (Acien et al., 2010; Dormido et al., 2014)

\begin{tabular}{lll}
\hline Parameter & Value & Unit \\
\hline Culture flow rate & 91 & $\mathrm{GPM}$ \\
Column cross-sectional area & 0.126 & $\mathrm{~m}^{2}$ \\
Tube cross-sectional area & 0.00636 & $\mathrm{~m}^{2}$ \\
Culture velocity in tube & 0.9 & $\mathrm{~m} / \mathrm{s}$ \\
Culture velocity in column & 0.0456 & $\mathrm{~m} / \mathrm{s}$ \\
Culture volume & 3.0 & $\mathrm{~m}^{3}$ \\
Tube length & 400 & $\mathrm{~m}$ \\
Tube diameter & 0.09 & $\mathrm{~m}$ \\
Column height & 3.5 & $\mathrm{~m}$ \\
Column diameter & 0.4 & $\mathrm{~m}$ \\
Reactor temperature & 35 & ${ }^{\circ} \mathrm{C}$ \\
Water density at $35^{\circ} \mathrm{C}$ & 994 & $\mathrm{Kg} \mathrm{m}^{-3}$ \\
Water dynamic viscosity at $35^{\circ} \mathrm{C}$ & 0.000719 & $\mathrm{~Pa}^{*} \mathrm{~s}$ \\
Mass flow rate & 5.69 & $\mathrm{Kg} \mathrm{s}^{-1}$ \\
Operation time & 24 & $\mathrm{hrs} \mathrm{day}^{-1}$ \\
Number of $90^{\circ}$ elbows & 37 & \\
\hline
\end{tabular}


Table 3.5. Assumptions used to calculate the specific power input of the centrifugal pump of the Almeria PBR (Norsker et al., 2012; Geankoplis, 2003)

\begin{tabular}{lll}
\hline Parameter & Value & Unit \\
\hline Tube roughness & 0.0015 & $\mathrm{~m}$ \\
Column roughness & 0.0015 & $\mathrm{~m}$ \\
Pump efficiency & 75 & $\%$ \\
\hline
\end{tabular}

The specifications and assumptions on Tables 3.4 and 3.5 were used along with the following method to calculate friction losses with a pump published by Geankoplis (2003).

Step 1: The Reynolds number in the tubes and columns were calculated using Equation 4.7.

Step 2: The relative roughness for the tubes and columns were calculated as follows:

$$
\text { Relative roughness }=\frac{\epsilon}{D}
$$

where $\epsilon$ is the equivalent roughness and $D$ is the tube or column diameter.

Step 3: With the relative roughness and Reynolds number values in hand, the Fanning friction factor, $f$, was obtained from the well-known Moody Diagram (Geankoplis, 2003) for both the tube and the column. 
Step 4: The Fanning friction factors were used in conjunction with Equation 3.7 to calculate the friction loss in the tube and the column (Geankoplis, 2003):

$$
F_{f}=4 f \frac{L}{D} \frac{v^{2}}{2}
$$

where $F_{f}$ is the friction loss in the straight tube or column, $L$ is the total length of tube or column height, $D$ is the tube or column diameter, and $v$ is the culture velocity in the tube or column.

Step 5: The friction loss in the fittings was calculated as follows (Geankoplis, 2003):

$$
h_{f}=K_{f} \frac{v_{1}^{2}}{2}
$$

where $h_{f}$ is the friction loss in the fittings, $K_{f}$ is the loss factor for the fitting $\left(0.75\right.$ for $90^{\circ}$ elbows), and $v_{1}$ is the average velocity of the fluid in the tube or column leading to the fitting. Dormido et al. (2014) reported that each 3,000-L PBR unit in the Almeria facility has thirty seven 90-degree elbows and these are the major fittings.

Step 6: The friction loss due to fluid contraction and expansion was calculated as follows (Geankoplis, 2003):

$$
\begin{gathered}
h_{c}=0.55\left(1-\frac{A_{2}}{A_{1}}\right) \frac{v_{2}^{2}}{2 \alpha} \\
h_{e x}=\left(1-\frac{A_{1}}{A_{2}}\right) \frac{v_{1}^{2}}{2 \alpha}
\end{gathered}
$$


where $h_{c}$ is the friction loss due to fluid contraction, $h_{e x}$ is the friction loss due to fluid expansion, $\alpha=1.0$ for turbulent flow, $v_{l}$ is the upstream fluid velocity in the smaller area, $v_{2}$ is the downstream velocity, $A_{l}$ is the smaller cross-sectional area, and $A_{2}$ is the bigger cross-sectional area.

Step 7: The total frictional loss was then calculated as follows (Geankoplis, 2003):

$$
\Sigma F=F_{f}+h_{f}+h_{c}+h_{e x}
$$

Step 8: The well-known mechanical-energy balance equation was implemented to calculate the shaft work of the centrifugal pump as follows (Geankoplis, 2003):

$$
\frac{1}{2 \alpha}\left(v_{2 a v}^{2}-v_{1 a v}^{2}\right)+g\left(z_{2}-z_{1}\right)+\frac{\Delta P}{\rho}+\Sigma F+W_{s}=0
$$

where the first term (from left) is the change in kinetic energy of the fluid, the second term is the change in potential energy of the fluid, the third term is the net change in pressure of the fluid, $\Sigma F$ is the total friction loss, and $W_{s}$ is the pump shaft work. To simplify Equation 3.12 it must be noted that the change in velocity of the culture is negligible, the culture is circulating in a loop, and the reactor is a closed system. Hence it can be safely assumed that there is no change in kinetic energy, potential energy and pressure. Equation 3.12 simplifies as follows:

$$
\Sigma F=-W_{s}
$$


Step 9: The pump power needed was then calculated as follows (Geankoplis, 2003):

$$
W_{p}=-\left(\frac{-W_{s}}{\eta}\right) m
$$

where $W_{p}$ is the pump power needed (in Watts), $\eta$ is the pump efficiency, and $m$ is the mass flow rate.

Step 10: Finally, the specific power input was calculated by taking the pump power needed $\left(W_{p}\right)$ and dividing it by the total working volume $\left(2.8 \mathrm{~m}^{3}\right)$ to obtain the power supply in watts per $\mathrm{m}^{3}$ of reactor volume. The calculated specific power input with Equations 3.6 through 3.14 was $14 \mathrm{kWh} \mathrm{m}^{-3}$. Acien et al. (2012) reported the specific power input from a centrifugal pump to a 3,000-L PBR module as $8.0 \mathrm{kWh} \mathrm{m}^{-3}$ (Table 2.8). The main result of the calculation of the specific power input of the centrifugal pump is in Table 4.5 in the Results Chapter. Other results for this calculation are in Appendix E.

If it is desired to calculate the discharge pressure of a pump for such a system, Equation 3.15 for head losses must be applied (Geankoplis, 2003):

$$
P=H \rho g=\frac{\sum F}{g} \rho g
$$

where $P$ is the required discharge pressure (in Pa), $H$ is the total head loss due to frictional forces (in m), $\rho$ is the fluid density (in $\mathrm{Kg} \mathrm{m}^{-3}$ ) and $g$ is the gravitational acceleration constant $\left(9.81 \mathrm{~m} \mathrm{~s}^{-2}\right)$. 


\section{CHAPTER IV: RESULTS AND DISCUSSION}

\subsection{Fluid Dynamics and Mass Transfer Characterization}

For measurements of the mass transfer coefficient $\left(K_{L} a\right)$ measurements the reactor was filled up with tap water and dissolved oxygen was removed from the reactor by sparging with nitrogen until the dissolved oxygen concentration reached about $10 \%$ of saturation. Oxygen was then introduced in the reactor as compressed air using diffusers. Figure 4.1 depicts the actual measured re-oxygenation profile for an aeration rate of $0.2 \mathrm{v} / \mathrm{v} / \mathrm{min}$ as an example. In Figure 4.1 de-oxygenation occurs in the first 1300 seconds and reoxygenation begins at around 1300 seconds. It should be noted that no algae was present at the time of these measurements. Refer to Appendices $G$ through $P$ for all other dissolved oxygen profiles.

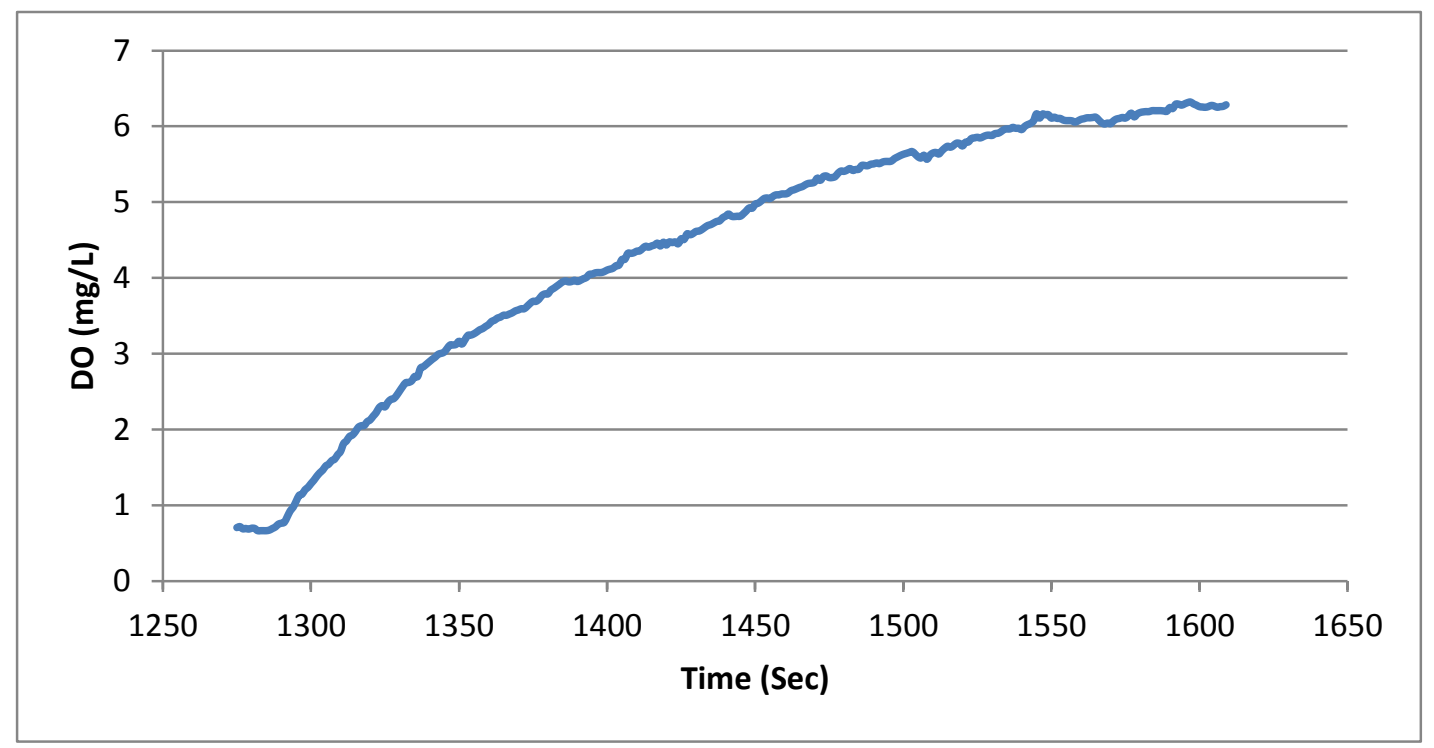

Figure 4.1. Re-oxygenation profile at $0.2 \mathrm{v} / \mathrm{v} / \mathrm{min}$ 
The critical limiting factor in aerated systems is the oxygen transfer rate (OTR), which should be optimized for ideal microalgae growth. The mass balance for dissolved oxygen, assuming a well-mixed liquid phase, is written as follows (Moutafchieva et al., 2013):

$$
\frac{d\left(C_{L}\right)}{d t}=K_{L} a\left(C_{L}^{*}-C_{L}\right)-r_{O_{2}}=O T R-O P R
$$

where $C_{L}$ represents the actual dissolved oxygen concentration in the liquid phase, $C_{L}{ }^{*}$ the dissolved oxygen concentration in equilibrium with the gas phase calculated according to Henry's law, and $K_{L} a$ the overall mass transfer coefficient. The oxygen transfer rate (OTR) is the rate at which oxygen is transferred from the liquid phase to the gas phase or vice versa (Moutafchieva et al., 2013). Whereas the oxygen production rate $(\mathrm{OPR})$ is the rate at which biomass produces oxygen to the liquid phase (Moutafchieva et al., 2013). When oxygen transfer is the rate-limiting step (i.e. due to low solubility of oxygen in water) and production of $\mathrm{O}_{2}$ by the microorganism is negligible, then $\mathrm{OPR}=0$ and the oxygen mass balance can be simplified to (Shuler \& Kargi, 2002, Sierra et al., 2008, Moutafchieva et al., 2013):

$$
\frac{d\left(C_{L}\right)}{d t}=K_{L} a\left(C_{L}^{*}-C_{L}\right)=O T R
$$

After integration and linearization Eq. 4.2 can be rearranged to obtain Eq. 4.3, which is used to calculate $K_{L} a$ as follows (Sierra et al., 2008):

$$
\ln \left(\frac{C_{L}^{*}-C_{L}}{C_{L}^{*}-C_{0}}\right)=-K_{L} a \cdot t
$$

where $C_{o}$ is the initial dissolved oxygen concentration at $\mathrm{t}=0$. 
Re-oxygenation profiles were all modeled according to Eq. 4.3 to obtain $K_{L} a$ values. As an example, Figure 4.2 shows the re-oxygenation profile for an aeration rate of 0.2 $\mathrm{v} / \mathrm{v} / \mathrm{min}$ modeled using a semi-log plot with Equation 4.3. From the slope in Figure 4.2, the value for $K_{L} a$ for this test is calculated to be $0.0039 \mathrm{~s}^{-1}$. The semi-log plot has a $\mathrm{R}^{2}$ value of 0.983; which means that the given linear relationship in the figure is a good fit.

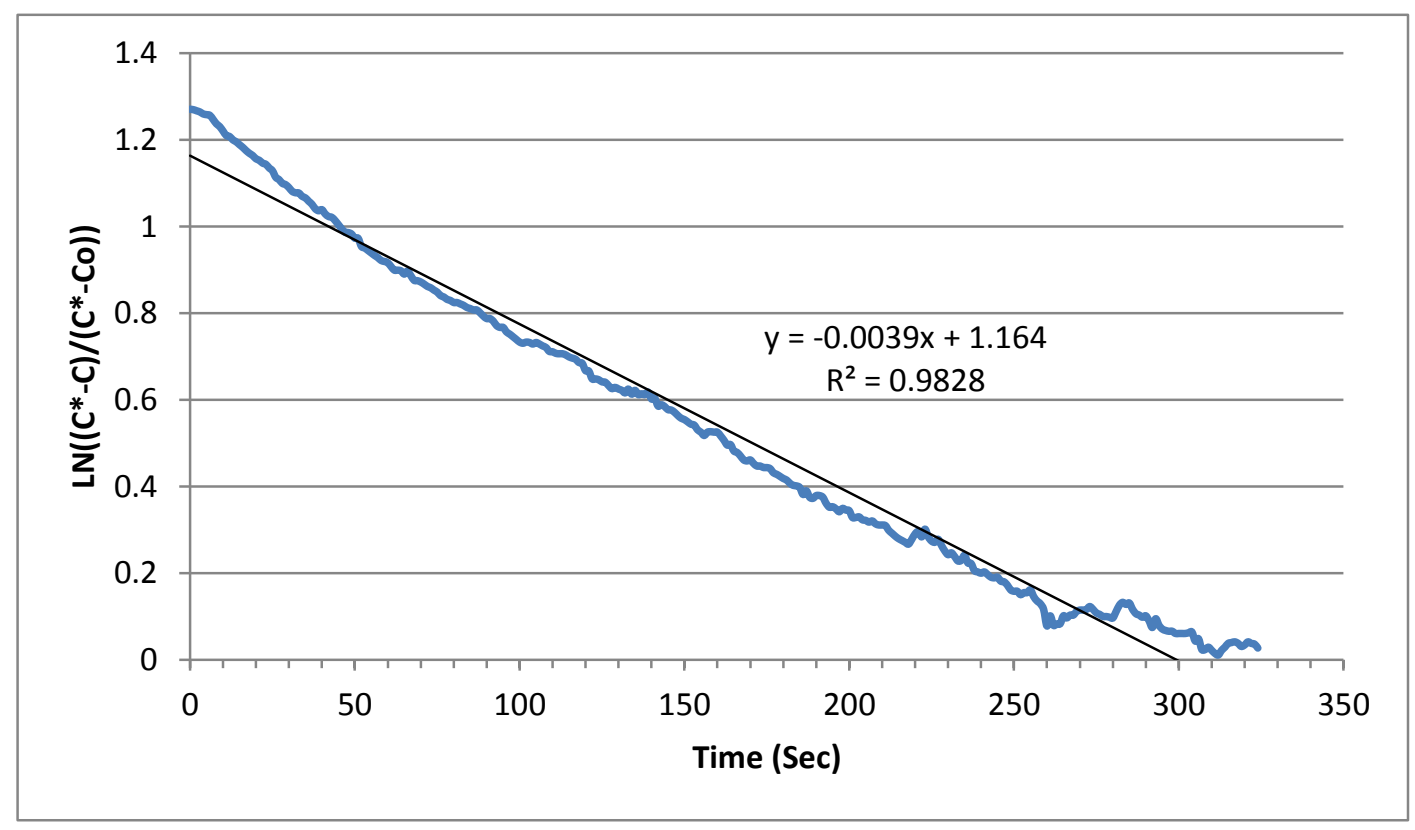

Figure 4.2. Re-oxygenation profile at $0.2 \mathrm{v} / \mathrm{v} / \mathrm{min}$ as modeled by Equation 4.3

The overall gas holdup, $\varepsilon$, was quantified employing the volume expansion method (Chisti, 1989) as follows:

$$
\varepsilon=\frac{h_{D}-h_{L}}{h_{D}}
$$

where $h_{L}$ is the measurement of unsaturated, static liquid height, and $h_{D}$ is the measurement of the height of gas-liquid dispersion upon aeration. 
The gas hold-up and overall oxygen transfer coefficient of the PBR increased linearly as aeration rate increased, and reached maximum values of 0.041 and $0.0092 \mathrm{~s}^{-1}$ respectively at the highest aeration rate of $0.5 \mathrm{v} / \mathrm{v} / \mathrm{min}$ (Figure 4.3). Sierra et al. (2008) reported similar profiles and capacities with a 250-L flat panel PBR equipped with a gas sparger. This flat-panel PBR has a maximum gas hold-up value of 0.018 and an oxygen transfer coefficient of $0.0063 \mathrm{~s}^{-1}$ at the highest aeration rate of $0.32 \mathrm{v} / \mathrm{v} / \mathrm{min}$ (Sierra et al., 2008). The prototype PBR resulted in similar values with an aeration rate of $0.32 \mathrm{v} / \mathrm{v} / \mathrm{min}$ (Figure 4.3). See Appendix D for data on Kla and gas holdup measurements.

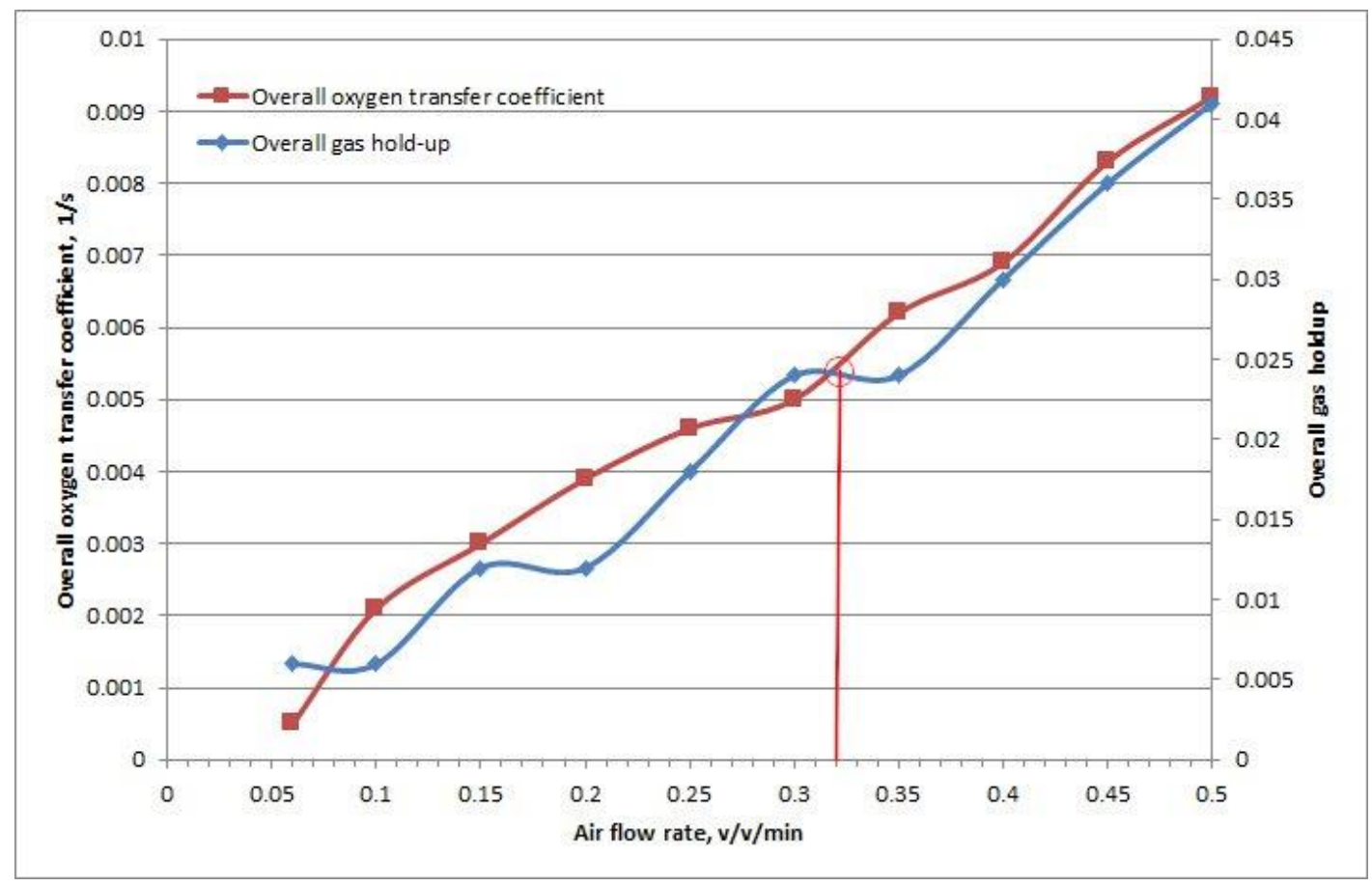

\section{Figure 4.3. Influence of the aeration rate on the gas holdup and overall oxygen transfer coefficient of the prototype PBR}

The gas holdup and overall mass transfer coefficient are important parameters in the design, operation and scale-up of aerated bioreactors. The gas holdup determines the 
residence time of the gas in the liquid phase and influences the gas-liquid interfacial area available for mass transfer (Chisti, 1989). The overall mass transfer coefficient is also a key variable. It is known that concentrations of photosynthetically-generated oxygen above air saturation generally inhibit photosynthesis in microalgae, which in turn limits the maximum productivity of microalgae in a bioreactor (Sierra et al., 2008). The determination of the overall mass transfer coefficient $\left(K_{L} a\right)$ thus is essential to provide an effective oxygen transfer rate from the liquid phase to the gas phase during algal growth and is often used to compare the efficiency of bioreactors (Moutafchieva et al., 2013).

Table 4.1 depicts published values of the overall mass transfer coefficient in some closed microalgal culture systems. A close look at this published data reveals that the overall mass transfer coefficient is normally in the magnitude range of $10^{-2}$ to $10^{-3} \mathrm{~s}^{-1}$, which coincides with the range of $K_{L} a$ values determined in the prototype PBR as shown in Figure 4.3. In conclusion, the prototype PBR provides adequate mass transfer without use of a recirculating pump.

Table 4.1. Comparison of overall mass transfer coefficients $\left(K_{L} a\right)$ in different closed microalgal culture systems (Ugwu et al., 2008; Xu et al., 2009)

\begin{tabular}{llll}
\hline Photobioreactor type & Volume $(\mathbf{L})$ & $\boldsymbol{K}_{\boldsymbol{L}} \boldsymbol{a}\left(\mathbf{s}^{\mathbf{- 1}}\right)$ & Algal strain \\
\hline Tubular reactor & 200 & 0.006 & Phaeodactylum \\
Tubular reactor & 75 & 0.004 & Phaeodactylum \\
Bubble column & 2 & $0.020-0.025$ & Phaeodactylum \\
Concentric tube airlift column & 12 & 0.020 & Phaeodactylum \\
Internally-illuminated column & 3 & 0.020 & Chlorella pyrenoidosa \\
Airlift tubular horizontal & 200 & 0.014 & Porphyridium cruentum \\
Bubble column & 13 & $0.002-0.005$ & Porphyridium sp. \\
External loop airlift column & 200 & 0.006 & Phaeodactylum \\
Inclined tubular reactor & 6 & 0.003 & Chlorella sorokiniana \\
Flat-plate reactor & 3 & 0.002 & Synechocystis aquatilis \\
Split-cylinder internal-loop airlift & 2 & 0.009 & Haematococcus pluvialis \\
\hline
\end{tabular}


As aforementioned, a tracer method was employed to determine liquid circulation velocity. From the measured time interval between the tracer peaks from the two $\mathrm{pH}$ electrodes and the known vertical distance between them, the linear liquid velocity in the tubes was calculated as follows (Chisti, 1989):

$$
U_{L}=\frac{d_{\text {electrode }}}{t_{\text {peaks }}}
$$

where $d_{\text {electrode }}$ is the known vertical distance between $\mathrm{pH}$ electrodes, $t_{\text {peaks }}$ is the measured time interval between tracer peaks, and $U_{L}$ is the liquid circulation velocity. $U_{L}$ was also measured for aeration rate intervals of $0.05 \mathrm{v} / \mathrm{v} / \mathrm{min}$. The liquid circulation velocity in the columns was then calculated using the law of conservation of mass, as follows (Geankoplis, 2003):

$$
U_{L 1} A_{1}=U_{L 2} A_{2}
$$

where $U_{L l}$ is the velocity of the culture in the tubes, $A_{l}$ is the cross sectional area of the tubes, $U_{L 2}$, is the velocity of the culture in the columns, and $A_{2}$ is the cross sectional area of the columns.

With data of velocity of the fluid in the tubes and columns, the Reynolds number for flow in tubes and columns was calculated as follows (Geankoplis, 2003):

$$
N_{R e}=\frac{D U_{L} \rho}{\mu}
$$

where $N_{R e}$ is the dimensionless Reynolds number, $D$ is the tube diameter, $U_{L}$ is the average velocity of the fluid, $\rho$ is the fluid density, and $\mu$ is the fluid dynamic viscosity. A 
Reynolds number below 2100 means the flow in the tubes is laminar, whereas above 2100 the flow is considered turbulent (Geankoplis, 2003). Turbulent flow is a good indicator that there is good mixing in the tubes and columns during fluid circulation (Geankoplis, 2003).

Reynolds number in the PBR tubes ranged from 4,000 to 17,000 (Figure 4.4), indicating highly turbulent flow. In the columns the Reynolds number was above 2,100 for all aeration rates above $0.2 \mathrm{v} / \mathrm{v} / \mathrm{min}$. This indicates that there is turbulent flow system-wide at aeration rates higher than $0.2 \mathrm{v} / \mathrm{v} / \mathrm{min}$ (Figure 4.4). In Figure 4.4 a sharp linear rise in the Reynolds number in the tube section is seen as the air flow rate increases, whereas the Reynolds number in the columns does not increase as fast but still follows a positive linear trend. However, the Reynolds number calculated for the columns does not take into account the mixing pattern created by the sparging of the air being diffused into the PBR through disc diffusers located in each of the two airlift columns. The air being sparged through the bubble columns adds significant mixing to the culture in the PBR. Refer to Appendix D for data on Reynolds number measurements. 


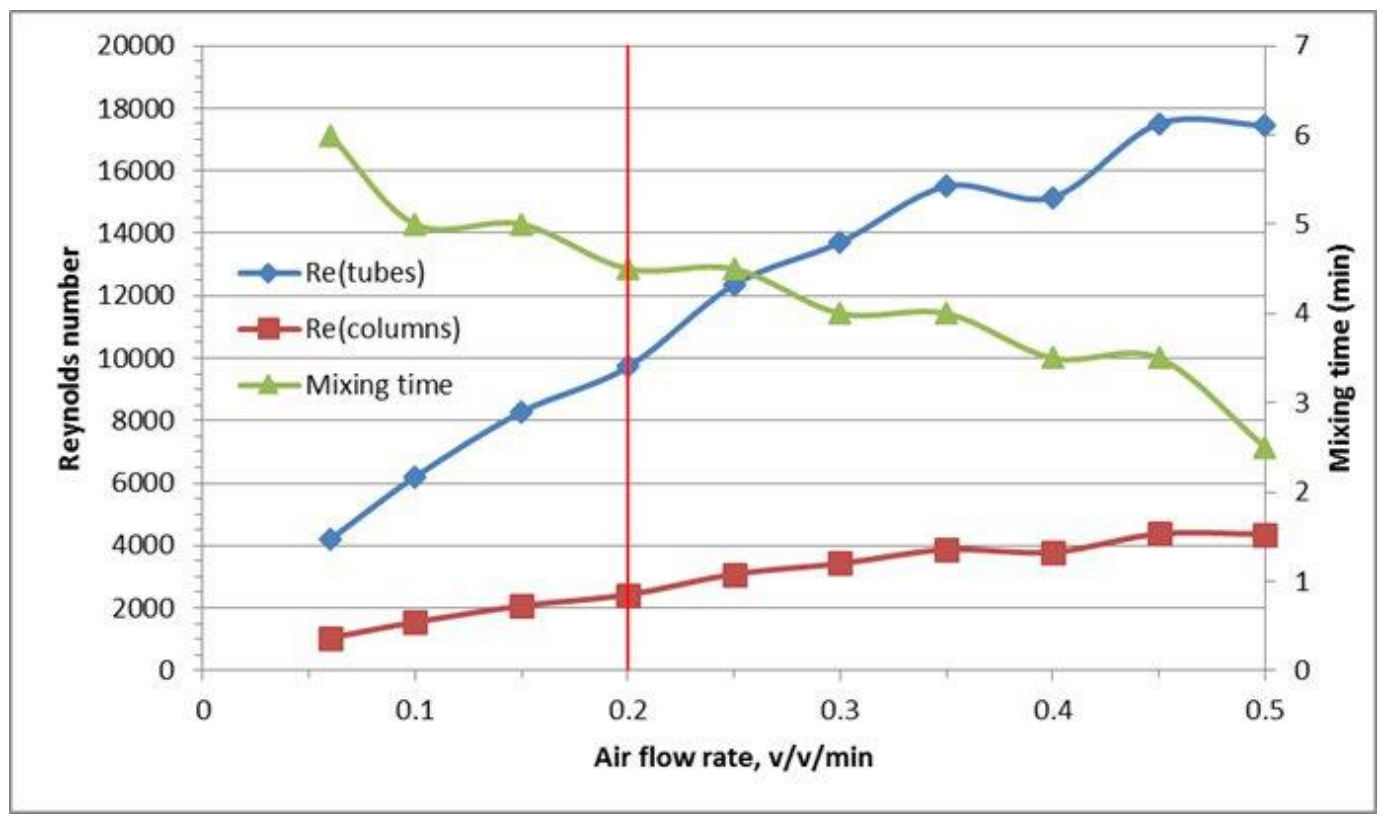

Figure 4.4. Influence of the aeration rate on the Reynolds number and mixing time

As mentioned in Section 3.6, mixing time is a direct indicator of mixing capacity of a reactor and is a useful parameter for comparing reactors in terms of characteristic mixing times (Sierra et al., 2008). The measured mixing time in the PBR was is in the range of 6 to 2.5 minutes for aeration rates between 0.06 and $0.5 \mathrm{v} / \mathrm{v} / \mathrm{min}$, as shown in Figure 4.4.

Sierra et al. (2008) reported mixing times of 1 min for bubble columns, 2 to 3 minutes for flat panel PBRs and 1 to $10 \mathrm{hrs}$ for tubular PBRs. From Figure 4.4 it can thus be concluded that there is good mixing in the entire reactor using aeration rates in the range of 0.2 to $0.5 \mathrm{v} / \mathrm{v} / \mathrm{min}$.

Aeration rate is also a key operating variable, influencing power consumption and resulting production cost, fluid dynamics of the system and other transport phenomena (Sierra et al., 2008). Determination of an optimum aeration rate is essential to maximize mixing and minimize both power consumption and shear stress to the cells. To choose an 
optimum aeration rate, the mass transfer capacity and Reynolds number profile were taken into account. Sierra et al. (2008) suggest that a mass transfer coefficient of $0.006 \mathrm{~s}^{-1}$ is sufficient for airlift columns to prevent over-accumulation of oxygen generated from photosynthesis, assuming a maximum biomass productivity of $2.0 \mathrm{~g} / \mathrm{L} \mathrm{d}^{-1}, 50 \%$ carbon content in the biomass, and a photosynthesis ratio of $1 \mathrm{~mol} \mathrm{O} / \mathrm{mol} \mathrm{CO}_{2}$. Xu et al. (2009) also reported that pneumatically agitated bubble columns and airlift devices should attain a mass transfer coefficient of $0.006 \mathrm{~s}^{-1}$, and culture circulation at low power consumption. Accomplishing these goals would make production of microalgae economically feasible in these types of PBRs (Xu et al., 2009). Based on this research done by Sierra et al. (2008) and $\mathrm{Xu}$ et al. (2009), an aeration rate of $0.35 \mathrm{v} / \mathrm{v} / \mathrm{min}$ was chosen as the optimum aeration rate to provide a required mass transfer coefficient of $0.006 \mathrm{~s}^{-1}$ and turbulent flow in the entire reactor (as seen in Figure 4.5). It should be noted that the mixing time at this aeration rate is about 4 minutes.

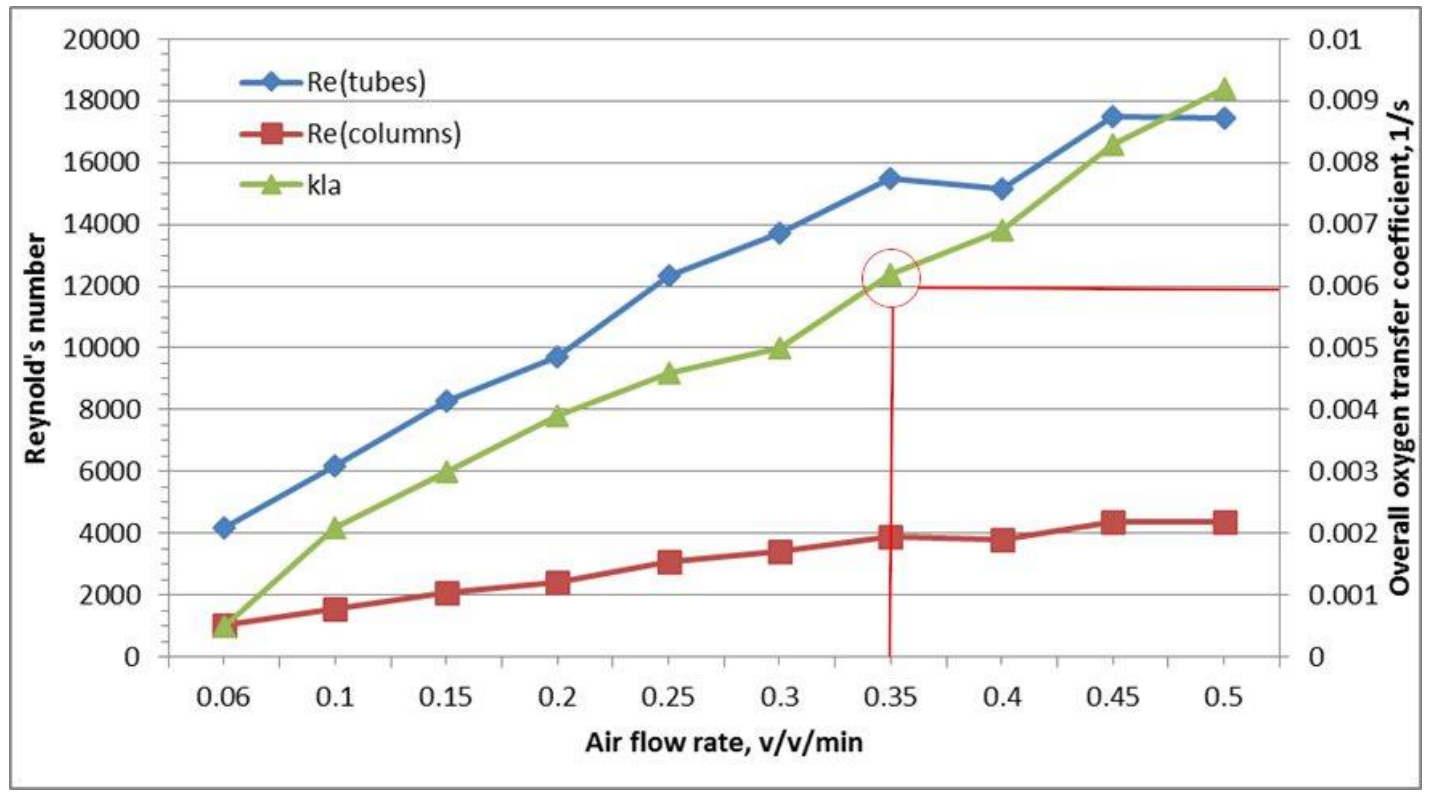

Figure 4.5. Influence of the aeration rate on the Reynolds number and overall oxygen transfer coefficient of the PBR 
The air blower power consumption for the prototype PBR was calculated using the linear relationship shown in Figure 3.23. From the slope of this line the specific power input due to circulation and gas exchange at the optimum aeration rate of $0.35 \mathrm{v} / \mathrm{v} / \mathrm{min}$ can be calculated using a basis of $1 \mathrm{~m}^{3}$ of culture as follows:

$$
1 \mathrm{~m}^{3} \text { culture } *\left(\frac{1000 \text { L culture }}{1 \mathrm{~m}^{3} \text { culture }}\right)\left(\frac{0.35 \text { L air } / \mathrm{min}}{\text { L culture }}\right)\left(\frac{1.0346 \mathrm{~W}}{\text { L air } / \mathrm{min}}\right)=362 \mathrm{~W}
$$

so the specific power input is $362 \mathrm{~W} \mathrm{~m}^{-3}$, or $8.7 \mathrm{kWh} \mathrm{m}^{-3}$ day $^{-1}$, assuming $24 \mathrm{hrs} \mathrm{day}^{-1}$ operation time at $0.35 \mathrm{v} / \mathrm{v} / \mathrm{min}$ aeration rate.

The measured magnitude of specific power input increases with increasing aeration and oxygen transfer capacity in a linear trend (Figure 4.6). According to this correlation, a specific power input of $362 \mathrm{~W} \mathrm{~m}^{-3}$ would provide optimum aeration rate of $0.35 \mathrm{v} / \mathrm{v} / \mathrm{min}$. This is very low compared to power consumption for tubular PBRs reported in the literature, which is a significant advantage for this PBR design. Sierra et al. (2008) reported that to attain the same mass transfer capacity, $40 \mathrm{~W} \mathrm{~m}^{-3}$ is required in bubble columns and 2400-3200 $\mathrm{W} \mathrm{m}^{-3}$ is required in tubular PBRs. The power consumption required by the prototype PBR $\left(362 \mathrm{~W} \mathrm{~m}^{-3}\right)$ is more than for bubble columns, but less than for tubular PBRs. Sierra et al. (2008) also reported that the highest specific power input in aerated systems range from $280 \mathrm{~W} \mathrm{~m}^{-3}$ for flat plate PBRs, to $200 \mathrm{~W} \mathrm{~m}^{-3}$ for bubble columns and airlift reactors, to $800-3400 \mathrm{~W} \mathrm{~m}^{-3}$ for tubular PBRs (Sierra et al., 2008). $362 \mathrm{~W} \mathrm{~m}^{-3}$ is the value that was used to calculate the specific biomass productivity (in $\mathrm{mg} \mathrm{kJ}^{-1}$ ) of the Cal Poly prototype PBR. See Appendix D for data on specific power input. 


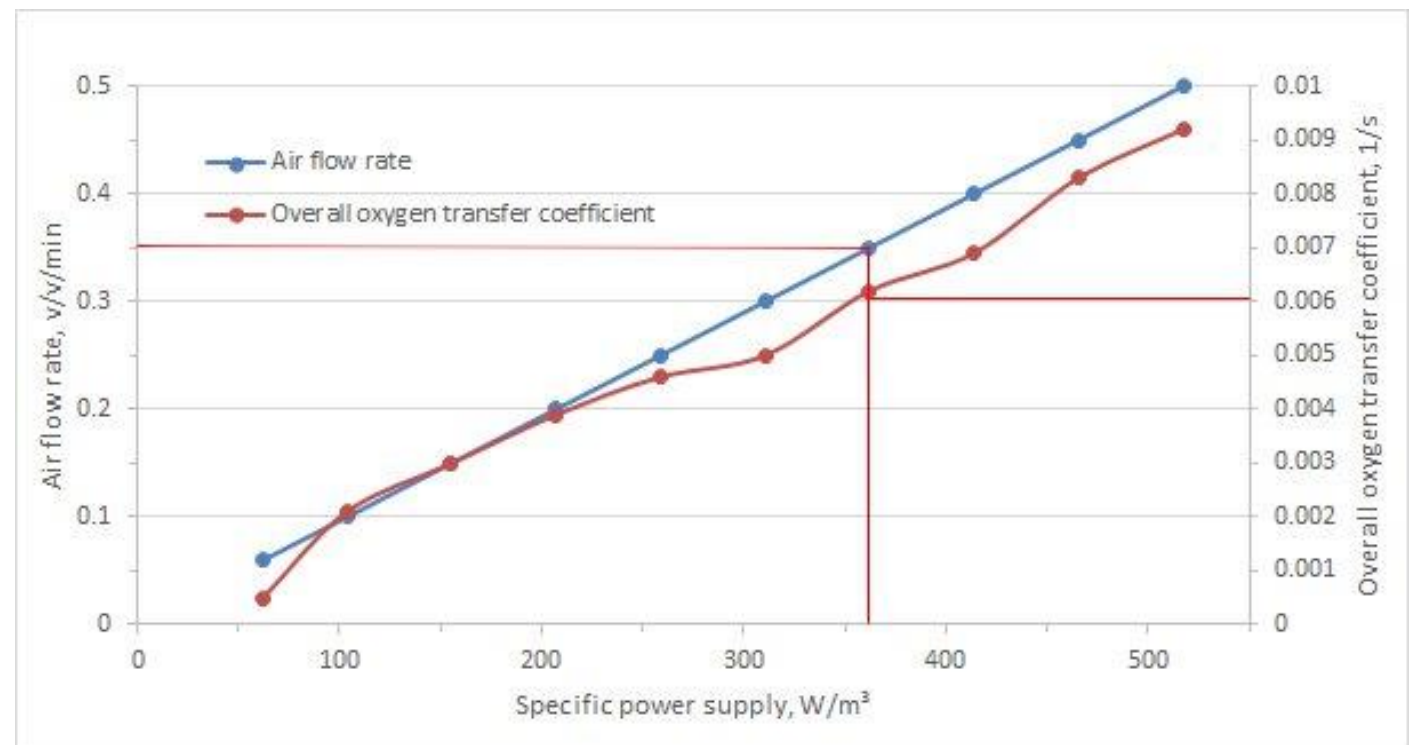

\section{Figure 4.6. Influence of specific power supply on aeration rate to the PBR and overall oxygen transfer coefficient}

The magnitude of the specific power input can also have a significant effect on shearsensitive microalgae since liquid turbulence increases with power input. When turbulence generates microeddies comparable to the size of cells, cell damage starts to occur (Sierra et al., 2008). Shear sensitive microalgae, such as Dunaliella tertiolecta and $P$.

tricornutum, can withstand up to 98 and $250 \mathrm{~W} \mathrm{~m}^{-3}$ respectively in bubble columns (Sierra et al., 2008). The model algae being used, Chlorella vulgaris, is not shear sensitive in the range of specific power supply studied in the prototype PBR because $C$. vulgaris can withstand up to $33,000 \mathrm{~W} \mathrm{~m}^{-3}$ in bubble columns (Bronnenmeier and Markl, 1982).

\subsubsection{Theoretical Scale-up of the Cal Poly Prototype PBR}

As determined above, the PBR prototype requires a specific power input of $362 \mathrm{~W} \mathrm{~m}^{-3}$ $\left(8.7 \mathrm{kWh} \mathrm{m}^{-3} \mathrm{day}^{-1}\right)$ to run at an air flow rate of $0.35 \mathrm{v} / \mathrm{v} / \mathrm{min}$ and maintain a required $K_{L} a$ 
value of $0.006 \mathrm{~s}^{-1}$. Since the total reactor volume is $235 \mathrm{~L}$ an aeration rate of $82 \mathrm{~L} \mathrm{~min}^{-1}$ was needed. To theoretically scale up the prototype PBR to 3,000 L the required air flow rate (in $\mathrm{L} \mathrm{min}^{-1}$ ) was calculated using $0.35 \mathrm{v} / \mathrm{v} / \mathrm{min}$ optimum aeration rate as follows:

$$
\text { Culture volume }(L) * \frac{0.35 \text { L air }}{\text { Lculture } * \text { min }}=\text { Required air flow rate }\left(\frac{\text { L air }}{\min }\right) \quad \text { Eq. } 4.8
$$

for scaling up to $3,000 \mathrm{~L}$ the required air flow rate is calculated as:

$$
(3,000 \text { L culture })\left(\frac{0.35 \mathrm{Lair} / \mathrm{min}}{\text { L culture }}\right)=1050{\mathrm{~L} \mathrm{air} \mathrm{min}^{-1}}^{-1}
$$

thus, for a 3,000 L unit, $1050 \mathrm{~L} \mathrm{~min}^{-1}$ of air would be required for culture circulation and gas exchange in the Cal Poly prototype PBR. The specific power input for circulation and gas exchange for a 3,000-L unit is:

$$
\left(\frac{3,000 \text { L culture }}{3 m^{3} \text { culture }}\right)\left(\frac{0.35 \mathrm{~L} \text { air } / \mathrm{min}}{L \text { culture }}\right)\left(\frac{1.0346 \mathrm{~W}}{\mathrm{~L} \text { air } / \min }\right)=362 \mathrm{~W} \mathrm{m^{-3 }}
$$

or $8.7 \mathrm{kWh} \mathrm{m}^{-3}$ day $^{-1}$ (which is a constant, regardless of the volume of the PBR), assuming $24 \mathrm{hrs} \mathrm{day}^{-1}$ operation time.

To be able to determine the total head loss of the projected 3,000-L Cal Poly PBR with the second scale-up approach described in Section 3.8, the specifications and assumptions on Tables 4.2 and 4.3 were used. Dimensions calculated in the second scale-up approach and the method to calculate friction losses with a pump published by Geankoplis (2003) and described in Section 3.9.2 were also used. The calculated total head loss for the projected 3,000-L Cal Poly PBR was $0.79 \mathrm{~m}$ and the required discharge pressure $7750 \mathrm{~Pa}$ 
(78 mbar). Refer to Appendix F for head loss calculations for the projected 3,000-L Cal

Poly PBR.

Table 4.2. Specifications to calculate the specific power input of the air blower for the projected 3,000-L Cal Poly PBR

\begin{tabular}{lll}
\hline Parameter & Value & Unit \\
\hline Culture flow rate in tubes and columns & 0.00081 & $\mathrm{~m}^{3} / \mathrm{s}$ \\
Column cross-sectional area & 0.126 & $\mathrm{~m}^{2}$ \\
Tube cross-sectional area & 0.00454 & $\mathrm{~m}^{2}$ \\
Culture velocity in tube & 0.178 & $\mathrm{~m} / \mathrm{s}$ \\
Culture velocity in column & 0.0064 & $\mathrm{~m} / \mathrm{s}$ \\
Tube length & 440 & $\mathrm{~m}$ \\
Tube diameter & 0.076 & $\mathrm{~m}$ \\
Column length & 8 & $\mathrm{~m}$ \\
Column diameter & 0.4 & $\mathrm{~m}$ \\
Reactor temperature & 35 & ${ }^{\circ} \mathrm{C}$ \\
Fluid density at $35^{\circ} \mathrm{C}$ & 994 & $\mathrm{Kg} \mathrm{m}^{-3}$ \\
Fluid dynamic viscosity at $35^{\circ} \mathrm{C}$ & 0.000719 & $\mathrm{~Pa}^{*} \mathrm{~s}$ \\
Operation time & 24 & $\mathrm{hrs} \mathrm{day}^{-1}$ \\
Culture volume & 3.0 & $\mathrm{~m}^{3}$ \\
Number of $90^{\circ}$ elbows & 222 & \\
\hline
\end{tabular}

Table 4.3. Assumptions to calculate the specific power input of the air blower for the projected 3,000-L Cal Poly PBR (Fraenkel, 1986; Norsker et al., 2012)

\begin{tabular}{lll}
\hline Parameter & Value & Unit \\
\hline Tube roughness & 0.0015 & $\mathrm{~m}$ \\
Column roughness & 0.0015 & $\mathrm{~m}$ \\
Pump efficiency & 25 & $\%$ \\
\hline
\end{tabular}

Raico Engineers Inc. provides a high pressure blower (Figure 4.7) that provides the required discharge pressure of $78 \mathrm{mbar}$ and air flow rate of $1050 \mathrm{~L} \mathrm{~min}^{-1}$ for the $3,000-\mathrm{L}$ Cal Poly PBR. The specifications of this blower are listed in Table 4.4. If such a blower were to be used in the projected 3,000-L Cal Poly PBR, the specific power input for 
culture circulation and gas exchange would be $8.0 \mathrm{kWh} \mathrm{m}^{-3}$ day $^{-1}$, assuming $24 \mathrm{hrs} \mathrm{day}^{-1}$ operation time. Refer to Appendix R for calculations of specific power input.

Table 4.4. Turbine blower specifications (Raico Engineers Inc., 2014)

\begin{tabular}{|c|c|c|c|c|}
\hline Model No. & $\begin{array}{l}\text { Power rating } \\
(k W)\end{array}$ & $\begin{array}{l}\text { Max. flow } \\
\left(L \text { min }^{-1}\right)\end{array}$ & $\begin{array}{l}\text { Max. pressure } \\
\text { (mbar) }\end{array}$ & $\begin{array}{l}\text { Max. vacuum } \\
\text { (mbar) }\end{array}$ \\
\hline 21 & 0.25 & 1333 & 110 & 100 \\
\hline
\end{tabular}

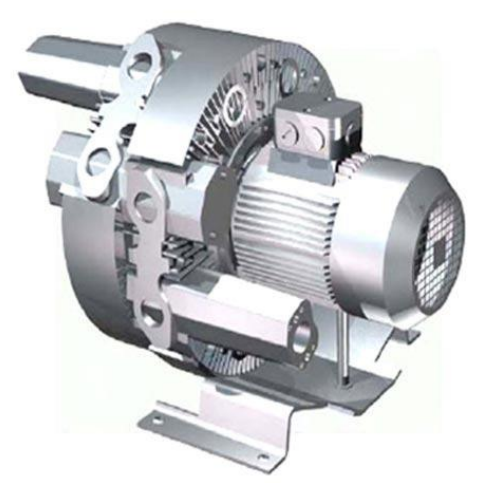

Figure 4.7. Turbine blower (Raico Engineers Inc., 2014)

\subsubsection{Comparison of Prototype PBR to Model System in Almeria (Spain) in Terms of Specific Power Input}

The plant located in Almeria (Spain) consists of 10 PBR units of 3,000 L each. Each of the 3,000-L units of the Almeria PBR facility shares a single air blower connected to the airlift column to mix the culture and strip the photosynthetically-generated oxygen, but each module uses its own centrifugal pump to circulate the culture around the array of tubes (Dormido et al., 2014). The total specific power consumption of the air blower and centrifugal pump for a 3,000-L unit in the Almeria facility was reported by Acien et al. 
(2012) to be $11 \mathrm{kWh} \mathrm{m}^{-3} \mathrm{day}^{-1}$. The total specific power consumption of the air blower and centrifugal pump for a 3,000-L unit in the Almeria facility as calculated using the assumptions and specifications found in the literature (see Tables 3.3, 3.4, and 3.5) was $20 \mathrm{kWh} \mathrm{m}^{-3}$ day $^{-1}$ (Table 4.5).

Table 4.5 compares the specific power supplies to run the reactors. Note that the Cal Poly prototype PBR needs 0.4 to $0.8 x$ (40 to $80 \%$ ) the total of the specific power input required by the Almeria PBR for culture circulation and gas exchange; based on calculated values and values obtain through literature review.

Table 4.5. Specific power input comparison between PBR units

\begin{tabular}{|c|c|c|c|c|c|}
\hline \multirow[t]{2}{*}{ Equipment } & \multicolumn{3}{|c|}{ 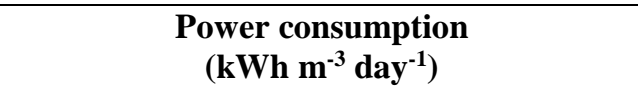 } & \multirow{2}{*}{$\begin{array}{l}\text { Ratio (Lit. } \\
\text { review values) } \\
\left(\frac{\text { Cal Poly }}{\text { Almeria }}\right)\end{array}$} & \multirow{2}{*}{$\begin{array}{l}\text { Ratio (Calc. } \\
\text { values) } \\
\left(\frac{\text { Cal Poly }}{\text { Almeria }}\right)\end{array}$} \\
\hline & $\begin{array}{l}\text { Cal Poly } \\
\text { Prototype } \\
\text { PBR }\end{array}$ & $\begin{array}{l}\text { Almeria PBR } \\
\text { (Lit. review } \\
\text { values) }\end{array}$ & $\begin{array}{l}\text { Almeria PBR } \\
\text { (Calculated } \\
\text { values) }\end{array}$ & & \\
\hline Air blower & $8.0^{\mathrm{b}}-8.7^{\mathrm{a}}$ & 3.2 & 5.5 & $3 \mathrm{x}$ & $2 \mathrm{x}$ \\
\hline Centrifugal pump & N/A & 8.0 & 14 & & \\
\hline Total & $8.0^{\mathrm{b}}-8.7^{\mathrm{a}}$ & 11 & 20 & $0.7-0.8 x$ & $0.4 \mathrm{x}$ \\
\hline
\end{tabular}

${ }^{\text {a }}$ Based on $1^{\text {st }}$ scale-up method

${ }^{\mathrm{b}}$ Based on $2^{\text {nd }}$ scale-up method

According to Molina et al. (2006), S. almeriensis was being cultured in the Almeria facility and would attain an annual maximum volumetric productivity of $0.2 \mathrm{~g} \mathrm{~L}^{-1} \mathrm{~d}^{-1}$. In spite of the high photosynthetic growth rate existing within the tubes dissolved oxygen levels would rise up to $400 \%$ of saturation. The main problem concerning the operation of the reactor was the excessive accumulation of dissolved oxygen at the end of the tubular loop. To decrease the residence time of the culture on the loop a centrifugal pump 
was installed. The use of a centrifugal pump increased the liquid velocity from $0.3 \mathrm{~m} / \mathrm{s}$ (airlift mode) to $0.9 \mathrm{~m} / \mathrm{s}$, causing a decrease of the residence time on the loop from 22 min (airlift mode) to only $7 \mathrm{~min}$. Moreover, the annual maximum volumetric productivity increased from $0.2 \mathrm{~g} \mathrm{~L}^{-1}$ day $^{-1}$ (airlift mode) to $0.6 \mathrm{~g} \mathrm{~L}^{-1}$ day $^{-1}$ by using the centrifugal pump. Acien et al. (2012) reported that in order to operate the PBRs in airlift mode and achieve the same annual maximum volumetric productivity $\left(0.6 \mathrm{~g} \mathrm{~L}^{-1} \mathrm{day}^{-1}\right)$ the reactors must be redesigned because the height of the airlift columns must be at least $4 \mathrm{~m}$ higher than height of the tubular photo-stages.

\subsection{Biomass Productivity of the Prototype PBR}

The prototype PBR was operated with two experimental runs to determine if the system could sustain a pilot-scale operation and to determine productivity. Biomass productivity of the prototype was analyzed by growing Chlorella vulgaris as the model algae.

\subsubsection{Operation of Batch Run \#1}

In the first experimental run, data were collected over a 28-day period of batch growth of Chlorella vulgaris in the prototype PBR. Parameters that were measured over the 28-day period were $\mathrm{pH}$, temperature, nitrate $\left(\mathrm{N}-\mathrm{NO}_{3}\right)$ concentration, and cell concentration.

Figure 4.8 shows the PBR under direct sunlight at the time of inoculation, which took place on Aug 5, 2013. The cell concentration in the PBR at the time of the inoculation was found to be $4.6 \times 10^{5}$ cells $\mathrm{mL}^{-1}$. 


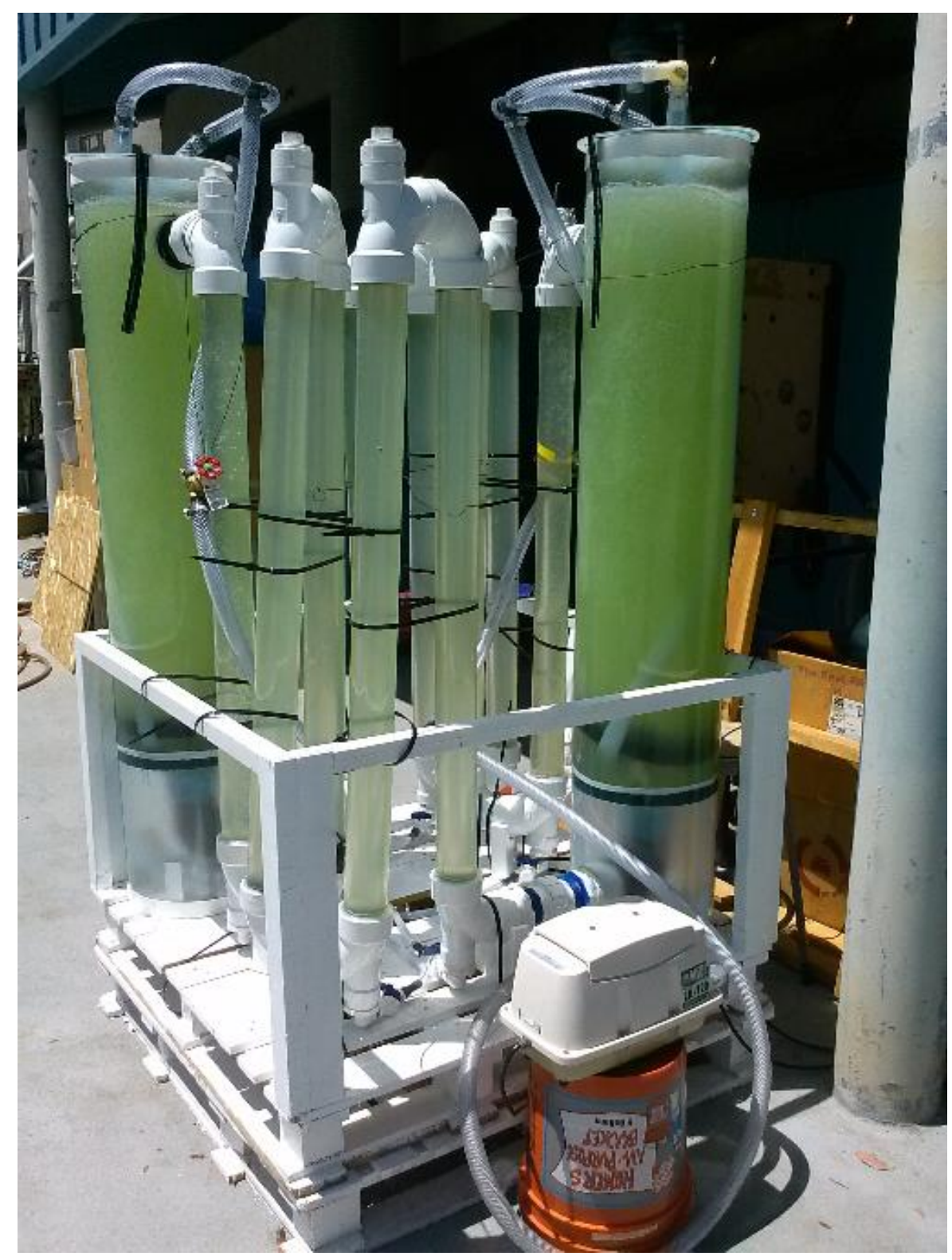

Figure 4.8. PBR at time of inoculation

During the first week of the first run, the algae appeared yellow, as seen in Figure 4.9. The cell concentration on Day 7 was found to be $4.5 \times 10^{6}$ cells $\mathrm{mL}^{-1}$, which means that the algae culture grew 10-fold (10x) in 7 days as compared to the cell concentration found on the PBR on the day of its inoculation $\left(4.6 \times 10^{5}\right.$ cells $\left.\mathrm{mL}^{-1}\right)$. The yellow color may be attributed to a relatively long lag phase; to be discussed in Section 4.2.4. 


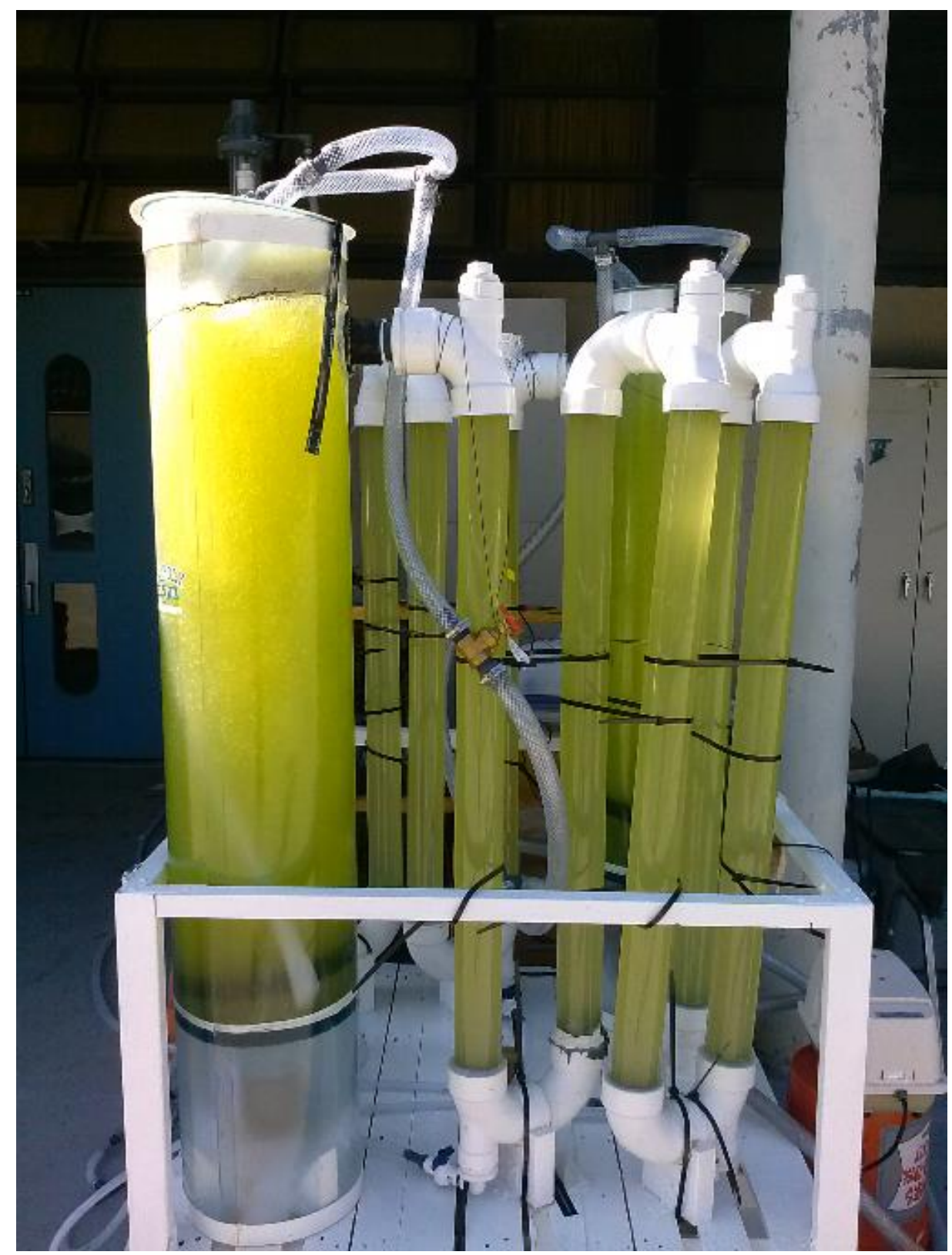

Figure 4.9. PBR after 7 days of inoculation

By Day 14 after inoculation, the algae culture was dark green (Figure 4.10) and the cell concentration in the PBR was found to be $1.10 \times 10^{7}$ cells $\mathrm{mL}^{-1}$. 


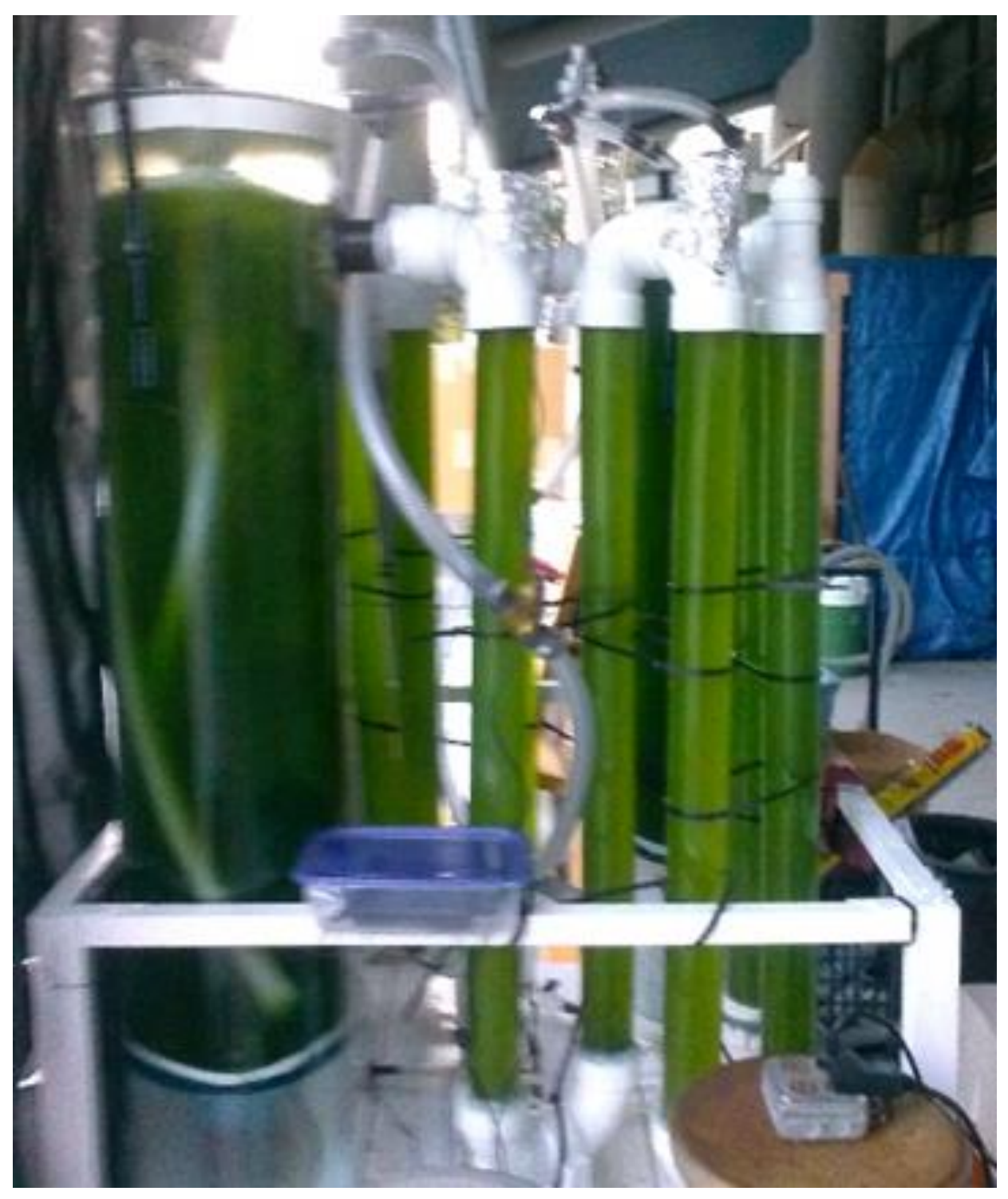

Figure 4.10. PBR after 14 days of inoculation

As seen below in Figure 4.11 immediately after inoculation, PBR cell concentration was about $4.6 \times 10^{5}$ cells $\mathrm{mL}^{-1}$ and after 28 days of data collection the cell concentration reached a high of about $3.0 \times 10^{7}$ cells $\mathrm{mL}^{-1}$. Figure 4.11 also depicts the 10 -fold increase in biomass concentration in the first 7 days of culture, a 6-fold increase in biomass concentration from Day 7 until Day 16, and a stationary or stagnant phase from Day 16 until Day 28. Overall, a 60-fold (60x) increase in biomass concentration was achieved 
from inoculation of the microalgae in the PBR until the start of the stationary phase at Day 16. Nitrate $\left(\mathrm{N}-\mathrm{NO}_{3}\right)$ concentration decreased rapidly over the first 18 days of culture, and about $15 \% \mathrm{~N}-\mathrm{NO}_{3}$ was left on Day 28 from the initial $\mathrm{N}-\mathrm{NO}_{3}$ used for inoculation. These data suggest that nitrogen deficiency was not an issue in this case.

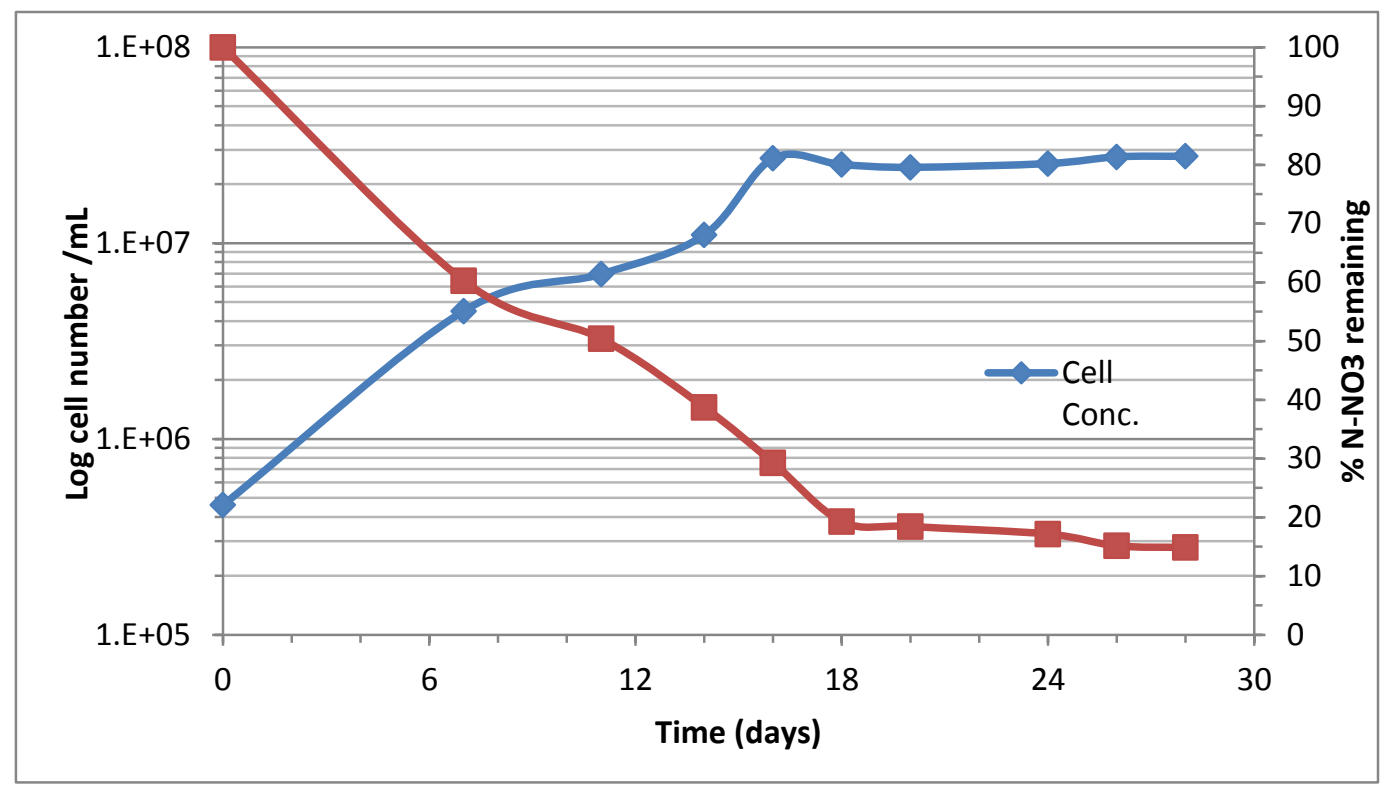

Figure 4.11. $\mathrm{N}-\mathrm{NO}_{3}$ and cell concentration data measured over 28 days

Figure 4.12 shows the temperature profiles of water in the PBR and ambient temperature collected over a 3-day period (Aug $5^{\text {th }}-$ Aug $8^{\text {th }}, 2013$ ) with the PBR under direct sunlight exposure. Figure 4.13 depicts the temperature profile of the water inside the PBR collected over a 19-day period (Aug $15^{\text {th }}-$ Sept $2^{\text {nd }}, 2013$ ) also with the PBR under direct sunlight exposure. By looking at Figures 4.12 and 4.13 it is seen that under direct sunlight exposure the maximum water temperature would be as high as $29^{\circ} \mathrm{C}$, discounting one high temperature on Day 25 , and as low as $16^{\circ} \mathrm{C}$, with a temperature fluctuation on average of about $10^{\circ} \mathrm{C}$ over each 24 -hour period. The average water temperature was 
about $22^{\circ} \mathrm{C}$. A study on the growth of $C$. vulgaris reported that the optimum temperature range to grow $C$. vulgaris is $30-35^{\circ} \mathrm{C}$ (Barghbani et al., 2012), and thus the average temperature in the PBR was about 8 degrees below this reported optimum temperature range.

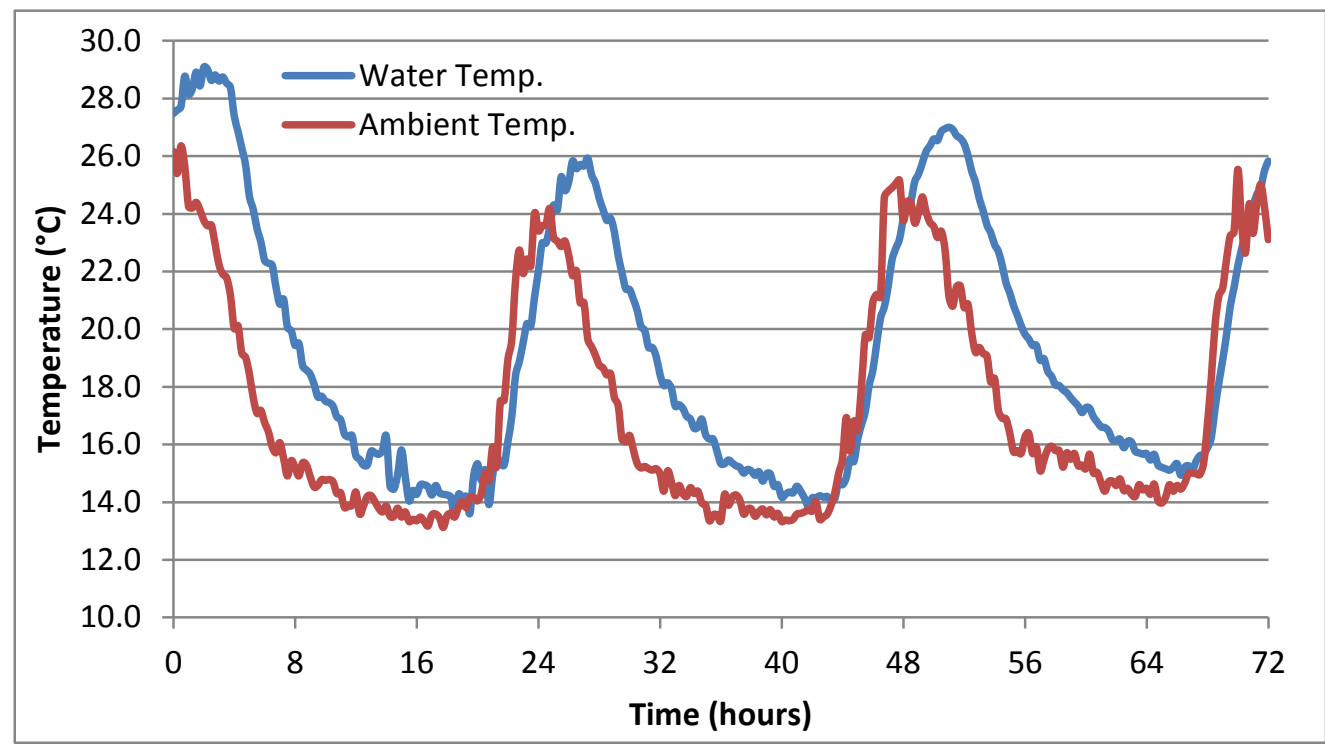

Figure 4.12. PBR temperature profile measured over 3 days

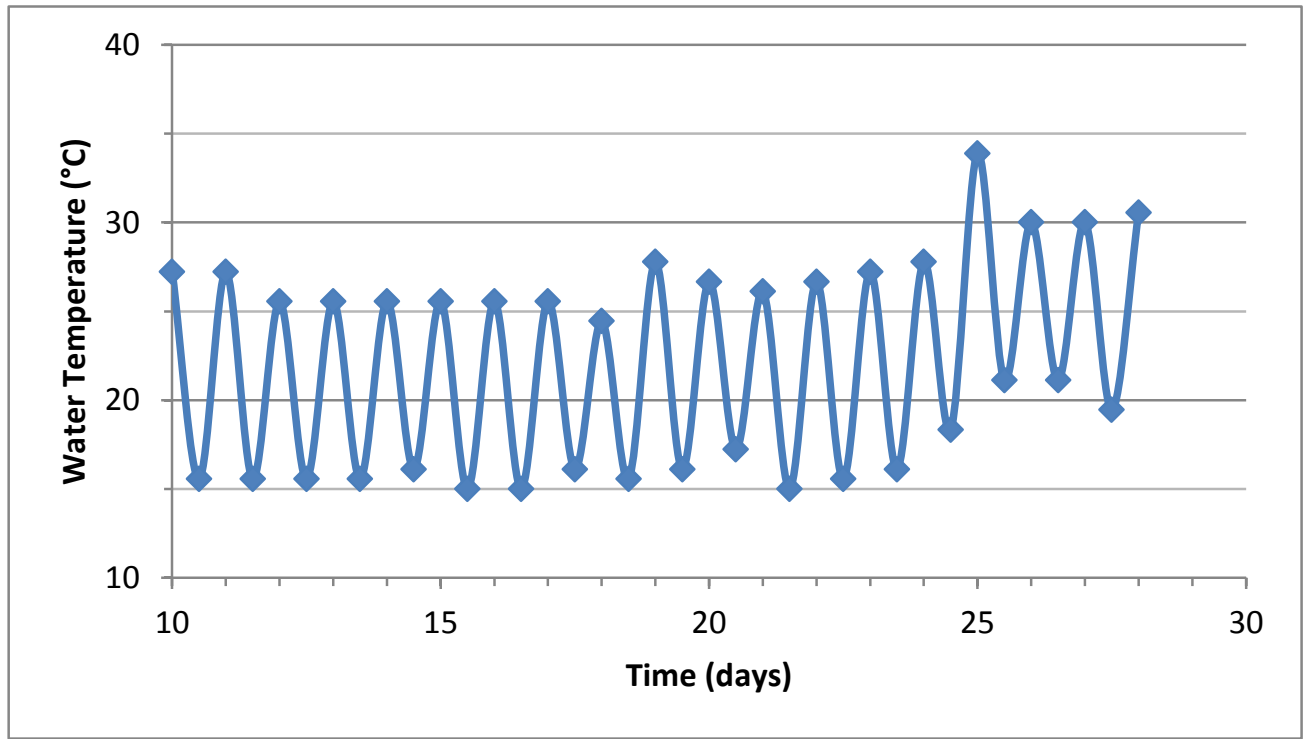

Figure 4.13. Temperature profile of the PBR liquid measured over 19 days 
The $\mathrm{pH}$ increased slightly from 8.0 to 8.5 over a period of 21 days (Aug $13^{\text {th }}-$ Sept $2^{\text {nd }}$, 2013) (Figure 4.14). Rachlin and Grosso (1991) reported that optimal growth of $C$.

vulgaris occurs in the $\mathrm{pH}$ range of 7.5 to 8.0 , which is slightly lower than the $\mathrm{pH}$ range of 8.0 to 8.5 seen in this first run (Figure 4.14).

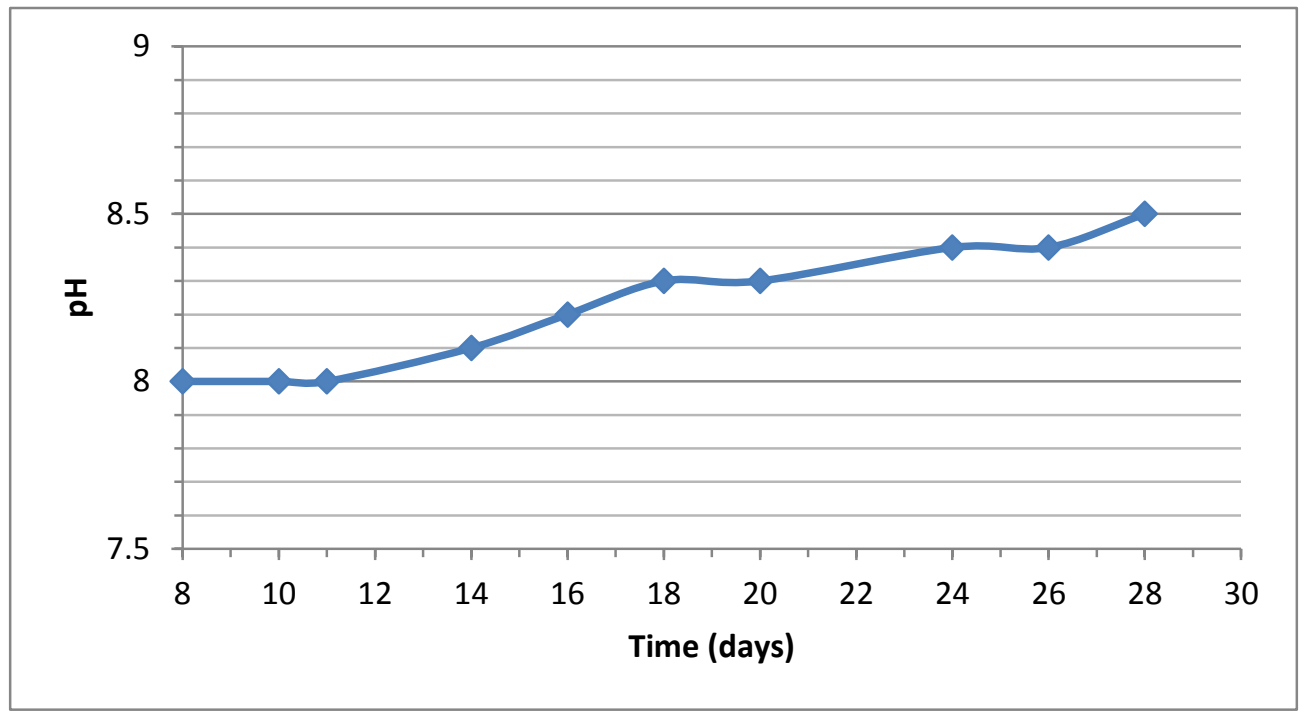

Figure 4.14. pH profile of the PBR measured over 21 days

Clearly, the $\mathrm{pH}$ and temperature were not maintained at their optimum values (as reported in the literature) during this first run. These parameters were not controlled for this run, but control of these parameters could push the maximum cell concentration and growth rates higher. Such control was attempted for the second run.

\subsubsection{Operation of Batch Run \#2}

For the second batch of Chlorella vulgaris, data were collected over a period of 17 days. Parameters collected during this batch run were biomass dry weight, cell concentration, $\mathrm{pH}$, temperature, dissolved oxygen concentration, light intensity and nitrate $\left(\mathrm{N}-\mathrm{NO}_{3}\right)$ 
concentration. To inoculate the prototype PBR the same procedure was used as in Batch Run \#1. This time injection of pure $\mathrm{CO}_{2}$ into the PBR on demand was used for $\mathrm{pH}$ regulation and to provide a source richer in carbon than is found in air. Also, average culture temperature was attempted to be increased for this second batch by using two ViaAqua ${ }^{\circledR}$ Quartz Water Heaters (Figure 3.10) rated at 300 Watts each. This additional effort was made in hopes of getting a higher maximum biomass concentration in a shorter period of time.

Figure 4.15 clearly shows a change in color from Day 0 to Day 17 in the PBR. Color changes were similar to those observed in Batch Run \#1, with a very yellow culture one week after inoculation. The yellow color may be attributed to a relatively long lag phase; to be discussed in Section 4.2.4. 

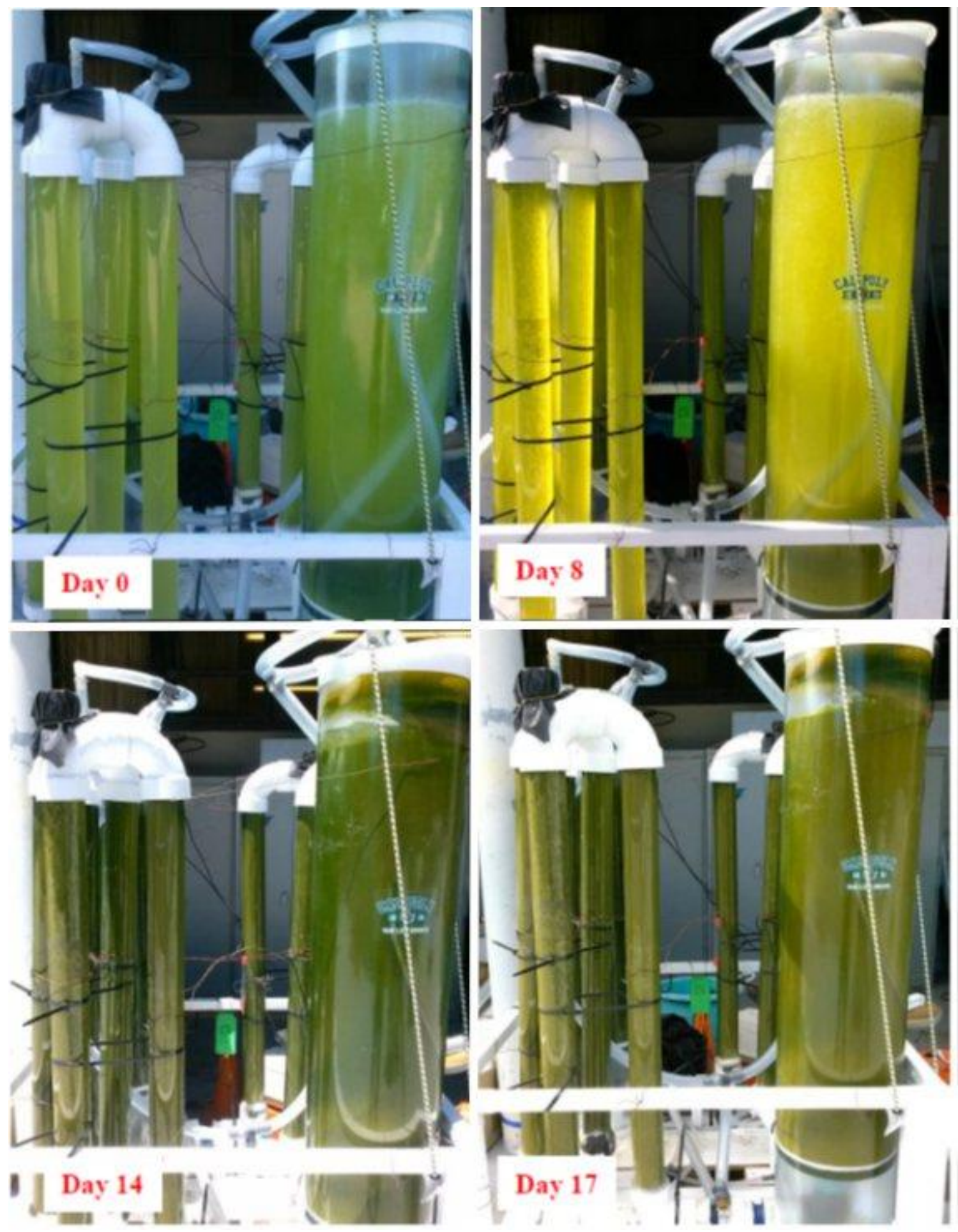

Figure 4.15. Growing the $2^{\text {nd }}$ batch of $C$. vulgaris for 17 days in the PBR 
On Day 0 after inoculation the cell concentration in the PBR was about $4.2 \times 10^{5}$ cells $\mathrm{mL}^{-}$ ${ }^{1}$ and by Day 17 the cell concentration was about $1.0 \times 10^{7}$ cells $\mathrm{mL}^{-1}$, with a high of $2.5 \times 10^{7}$ cells $\mathrm{mL}^{-1}$ on Day 14. Additional use of pure $\mathrm{CO}_{2}$ on this second run, along with better $\mathrm{pH}$ and temperature control, was not found to affect maximum biomass concentration but did squeeze the growth into a shorter period of time. Moreover, it appears that the lag phase for this run was longer than for Batch Run \#1 (Figure 4.16).

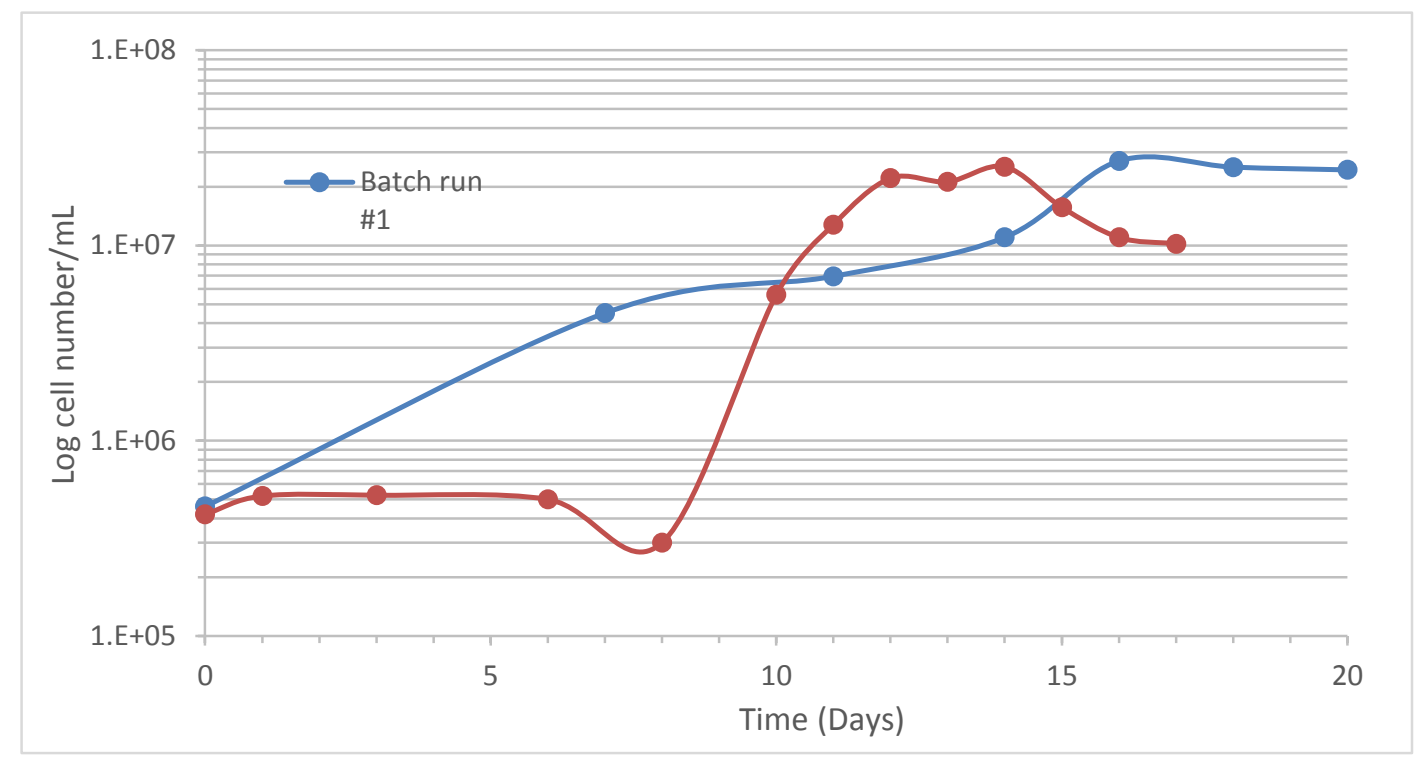

Figure 4.16. Cell concentration data measured for both batch runs

A lag phase of about 8 days was observed and exponential growth was observed from Day 8 to Day 12 and a stationary phase after Day 12 (Figure 4.17). The maximum cell concentration $\left(2.5 \times 10^{7}\right.$ cells $\left.\mathrm{mL}^{-1}\right)$ was reached at Day 14 and after Day 14 a death phase appears to begin. Thus, a 60-fold (60x) increase in cell concentration (also seen in Batch Run \#1) was observed from inoculation of the PBR (which began at $4.2 \times 10^{5}$ cells $\mathrm{mL}^{-1}$ ) until Day 14. Biomass concentration reached a maximum of about $1.0 \mathrm{~g} \mathrm{~L}^{-1}$ dry weight, and began Decreasing after day 14, which was similar to cell count behavior (Figure 
4.18). Moreover, it can be seen that $\mathrm{N}-\mathrm{NO}_{3}$ concentration fell steadily from inoculation day until day 17; reaching a low of $30 \%$ remaining $\mathrm{N}-\mathrm{NO}_{3}$.

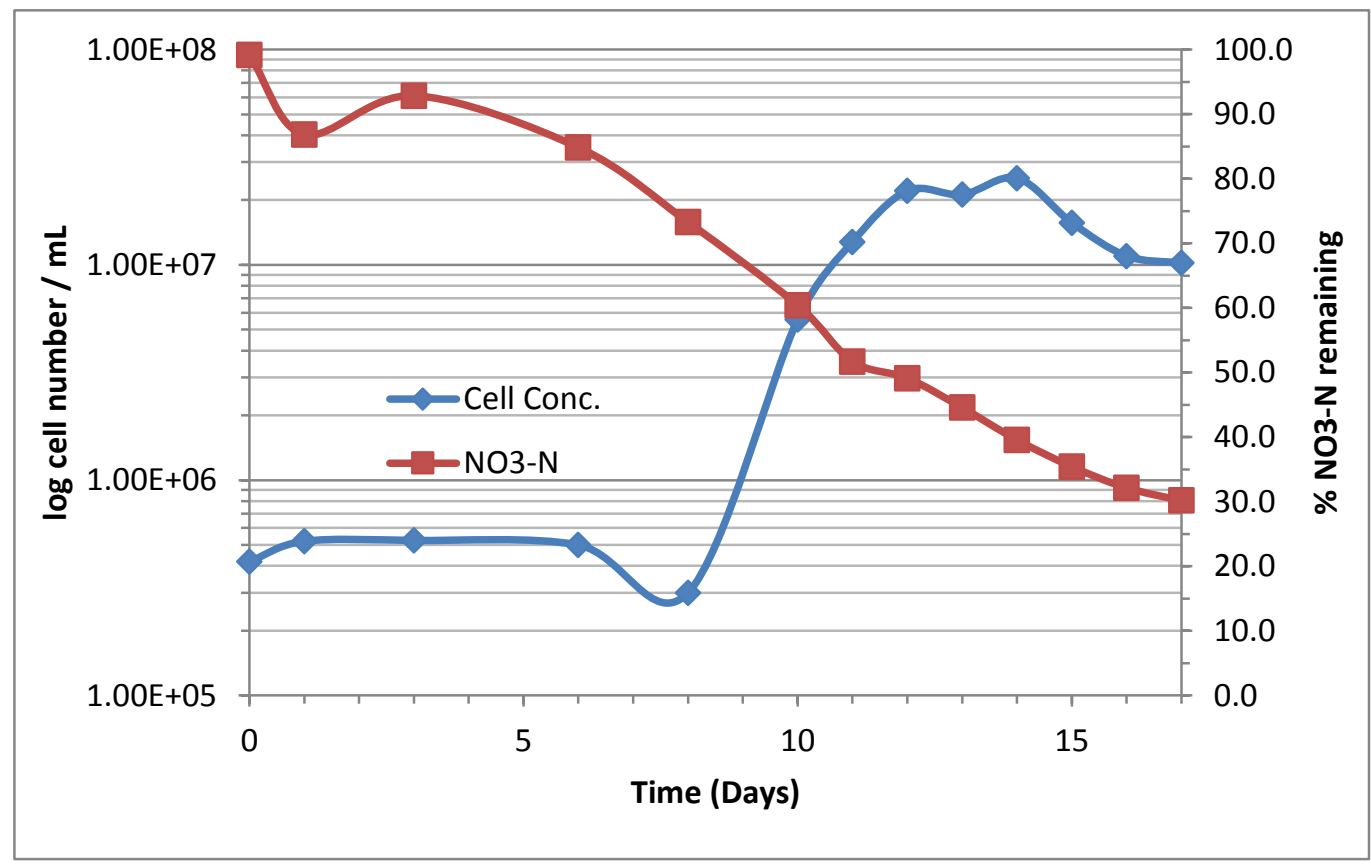

Figure 4.17. $\mathrm{N}-\mathrm{NO}_{3}$ and cell concentration data measured for Batch Run \#2

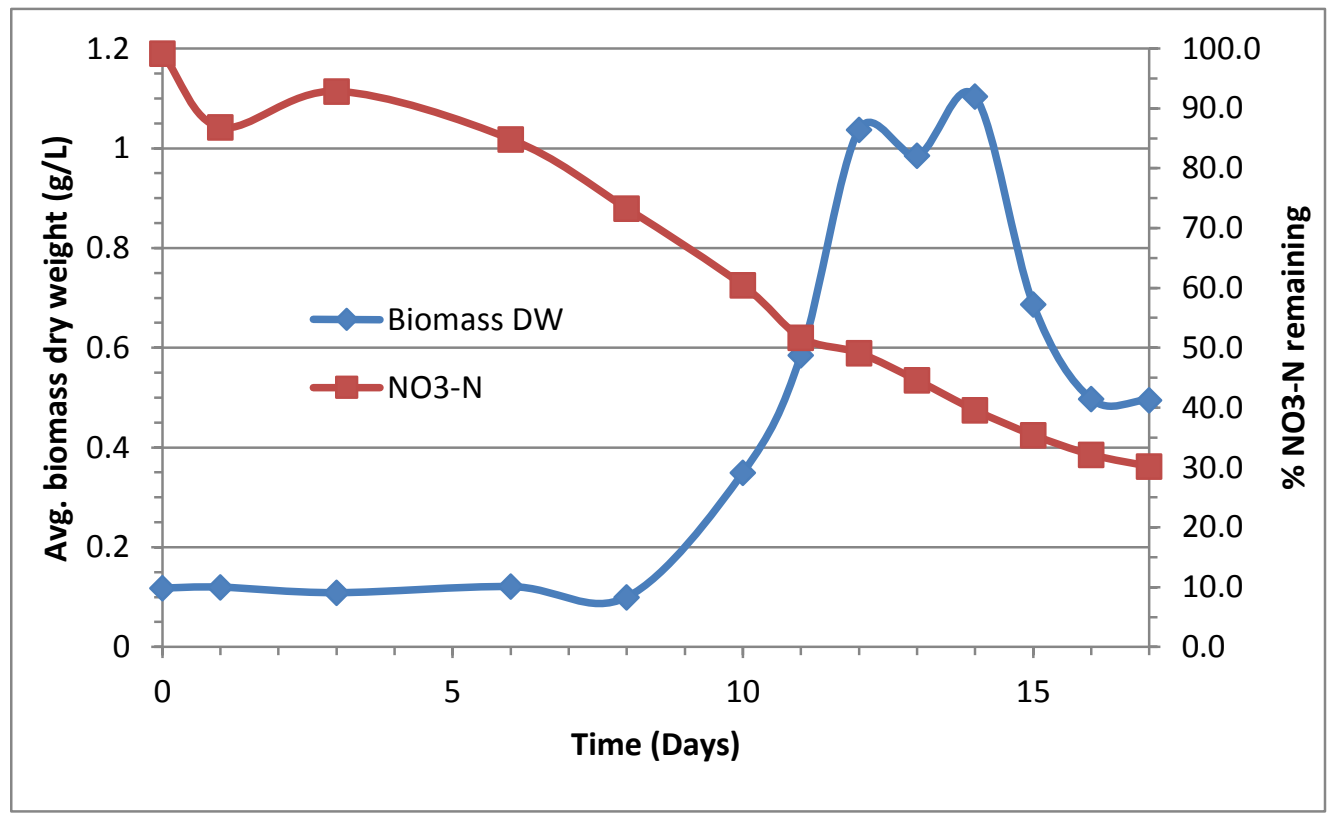

Figure 4.18. $\mathrm{N}-\mathrm{NO}_{3}$ concentration and average biomass dry weight measured for Batch Run \#2 
By looking closely at the data collected for Batch Run \#2 it can be seen that control of temperature and $\mathrm{pH}$ did increase the rate of biomass productivity. In Batch Run \#2 it took 12 days to get to the maximum cell concentration achieved in Batch Run \#1, compared to 16 days for Batch Run \#1 (Figure 4.16). Note that light intensity was an independent variable in this experiment because the culture was grown outdoors.

The relatively long lag phase (about one week) and yellow color observed in both runs is most likely due to a phenomenon called chlorosis (Figure 4.16). Chlorosis in algae is when cells or a culture of cells undergo a change in chlorophyll composition due to an inability to produce insufficient chlorophyll in their chloroplasts; the organelle responsible for conducting photosynthesis in algae (Pessarakli, 1999). Chlorophyll is the green photosynthetic pigment that gives the green color to algae. A stress condition on certain types of algae, such as Chlorella, may cause chlorosis in their chloroplasts as a response to resisting that stress condition for survival and trying to acclimate to that condition (Pessarakli, 1999). Stress on the cells can be due to nutrient imbalance, such as limitation of organic nitrogen and inorganic minerals, or environmental imbalance, such as temperature stress or light intensity stress (Pessarakli, 1999). Temperature stress can be due to large temperature fluctuations and light intensity stress can be due to lack of acclimation by the cells to certain intensity of light (Pessarakli, 1999). It is believed that not operating the unit in increasing amounts of light, as recommended for rapid cell acclimation, is what caused the chlorosis in the culture, hence the long lag phase of the culture. The PBR was exposed to direct sunlight after inoculation for both batch runs. Moreover, it is known that Chlorella has the ability to resist photoinhibition, which is a 
progressive inhibition of photosynthesis and eventual cell death by excessive light intensity (Pessarakli, 1999).

The average $\mathrm{pH}$ for the whole 17-day period of Batch Run \#2 was about 7.5 and varied from 6.5 to 8.0 except for a low excursion on Day 10 (Figure 4.19). This is slightly below the optimum $\mathrm{pH}$ range of 7.5 and 8.0 as reported for C. vulgaris by Rachlin and Grosso (1991).

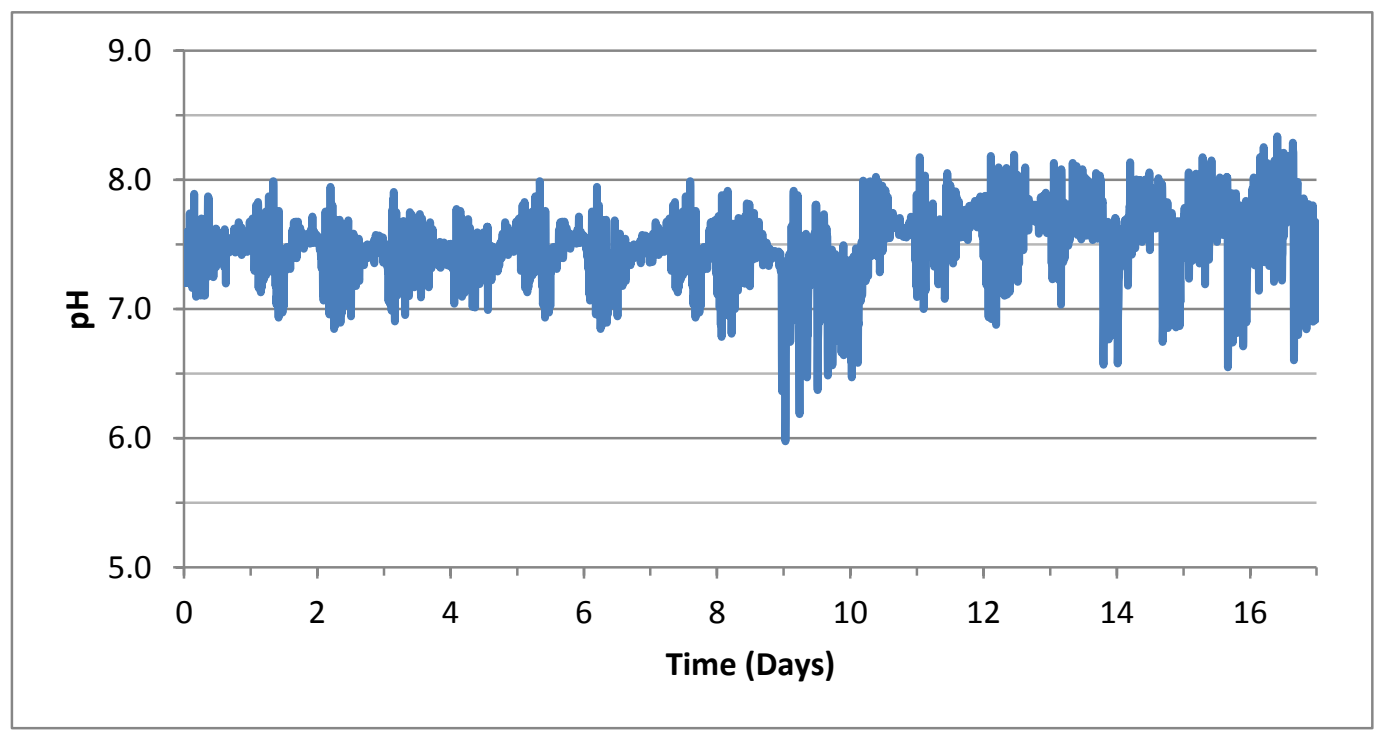

Figure 4.19. pH profile for Batch Run \#2 measured over 17 days

The average temperature for Batch Run $\# 2$ was $27^{\circ} \mathrm{C}$, but temperature varied daily between 21 and $32^{\circ} \mathrm{C}$ (Figure 4.20). This average does not fall within the optimum temperature range of $30-35^{\circ} \mathrm{C}$ reported by Barghbani et al. (2012) but is higher than the Batch Run \#1 average of $22{ }^{\circ} \mathrm{C}$, and thus closer to the optimum temperature range. 


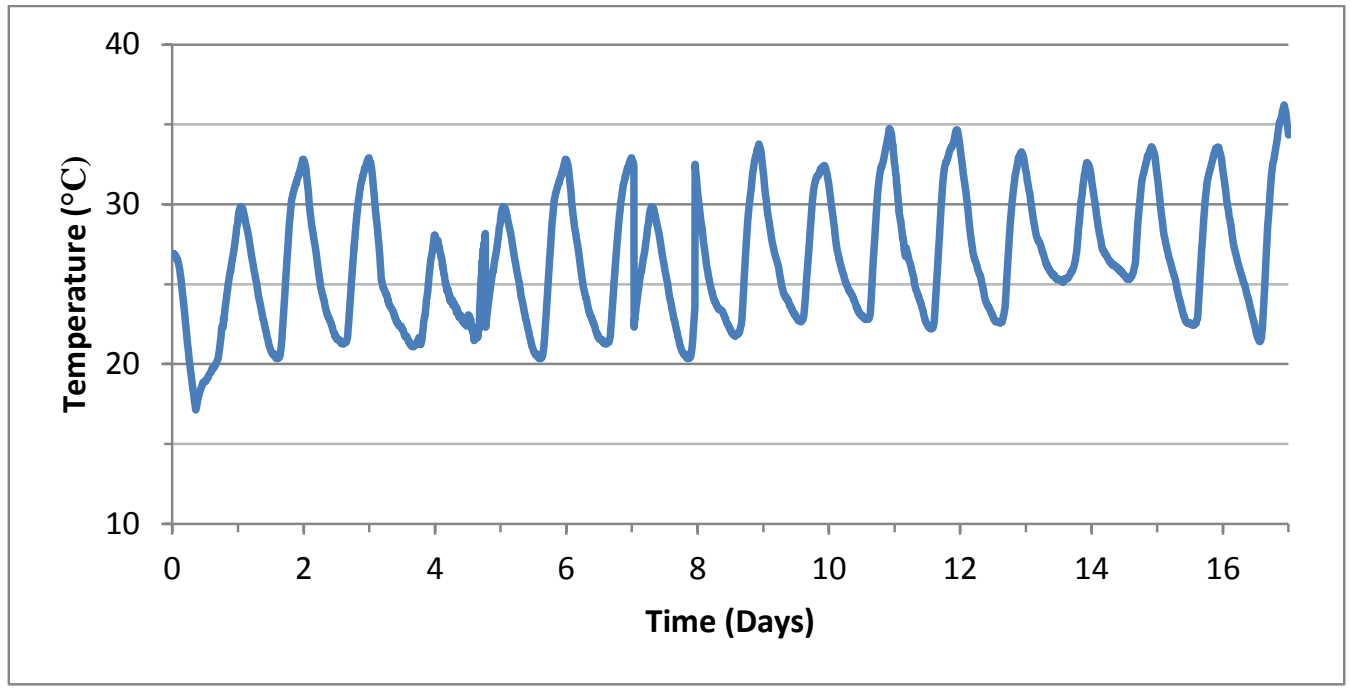

Figure 4.20. Temperature profile for Batch Run \#2 measured over 17 days

Light intensity impinging onto the reactor walls averaged 4100 lux and varied from zero at night to about 20,000 lux at peak sunlight (Figure 4.21). Oilgae (2013) reported an optimal illumination range of 5,000 to 10,000 lux for optimal algae growth in a photobioreactor. The average illumination seen in Figure 4.21 does not fall within this range, but illumination did fluctuate across this optimum range frequently over the 17 day period.

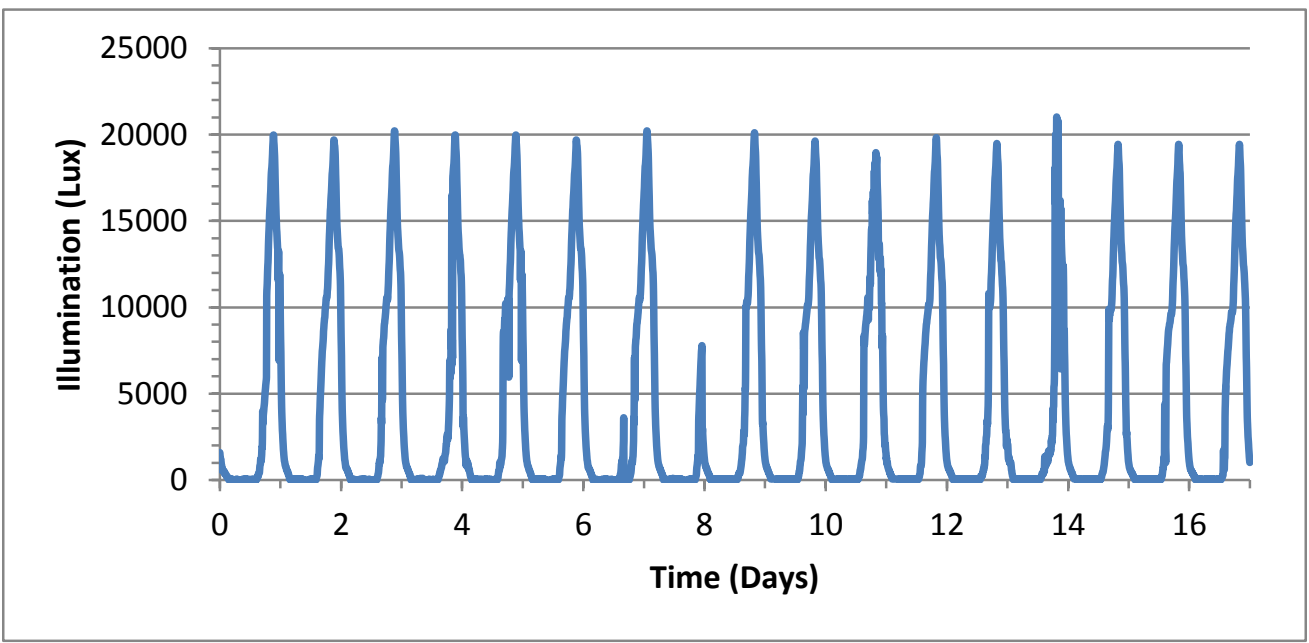

Figure 4.21. Illumination profile for Batch Run \#2 measured over 17 days 
The dissolved oxygen profile for Batch Run \#2 is depicted in Figure 4.22 as \% saturation, and averaged $100.7 \%$ saturation. Sierra et al. (2008) states that oxygen concentrations above saturation values (above 100\%Sat.) generally inhibit photosynthesis in microalgae. In this run the dissolved oxygen concentration did go above $100 \%$ Sat several times but not significantly. As seen in Figure 4.22, dissolved oxygen concentration experienced small fluctuations around $100 \%$ Sat during the entire run. Thus, we can conclude that dissolved oxygen toxicity was not likely an issue in growing this batch of C. vulgaris. Refer to Appendix $\mathrm{C}$ to see what times of day the dissolved oxygen was measured.

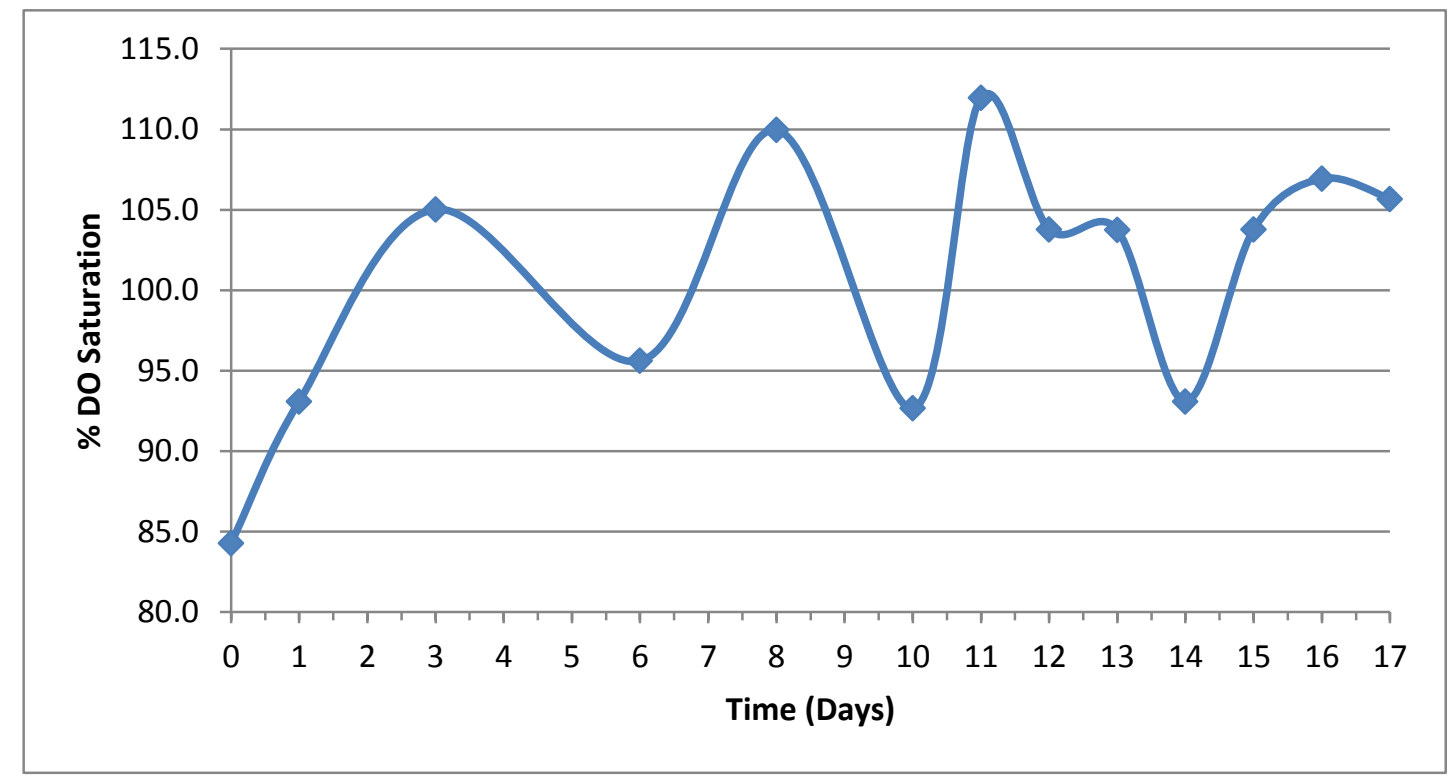

Figure 4.22. Dissolved oxygen profile for Batch Run \#2 as \%Sat

\subsubsection{Comparison of Prototype PBR to Model System in Almeria (Spain) in Terms}

\section{of Biomass Productivity}

To better compare the prototype PBR to the system in Almeria in terms of productivity, calculation of VP, OAP and ISP for the prototype PBR was carried out based on biomass 
dry weight (Figure 4.18) during exponential growth phase of Batch Run \#2 (from Day 8 to Day 12) and a method published by Shuler \& Kargi (2002):

Step 1: Recognizing that in the exponential growth phase or logarithmic growth phase the net specific growth rate determined by either cell number or cell mass is the same, and nutrient concentration is large and independent from growth rate, the exponential growth rate is first order (Shuler \& Kargi, 2002):

$$
\frac{d X}{d t}=\mu_{n e t} X, \quad X=X_{o} \quad \text { at } \quad t=0
$$

where $X$ is the active cell concentration at time $t, X o$ is the initial cell concentration at $t=$ 0 , and $\mu_{\text {net }}$ is the net specific growth rate.

Step 2: Integration of Eq. 4.11 yields:

$$
\ln \frac{X}{X_{o}}=\mu_{n e t} t
$$

Step 3: Modeling the data on biomass dry weight (Figure 4.18) of the exponential growth phase of Batch Run \#2 (from Day 8 to Day 12) according to Eq. 4.12 yields the semi-log plot shown in Figure 4.23. From the slope in Figure $4.23 \mu_{\text {net }}$ is calculated to be 0.5917 day $^{-1}$. The semi-log plot has a $R^{2}$ value of 0.998 ; which means that the given linear relationship in the figure is a good fit. 


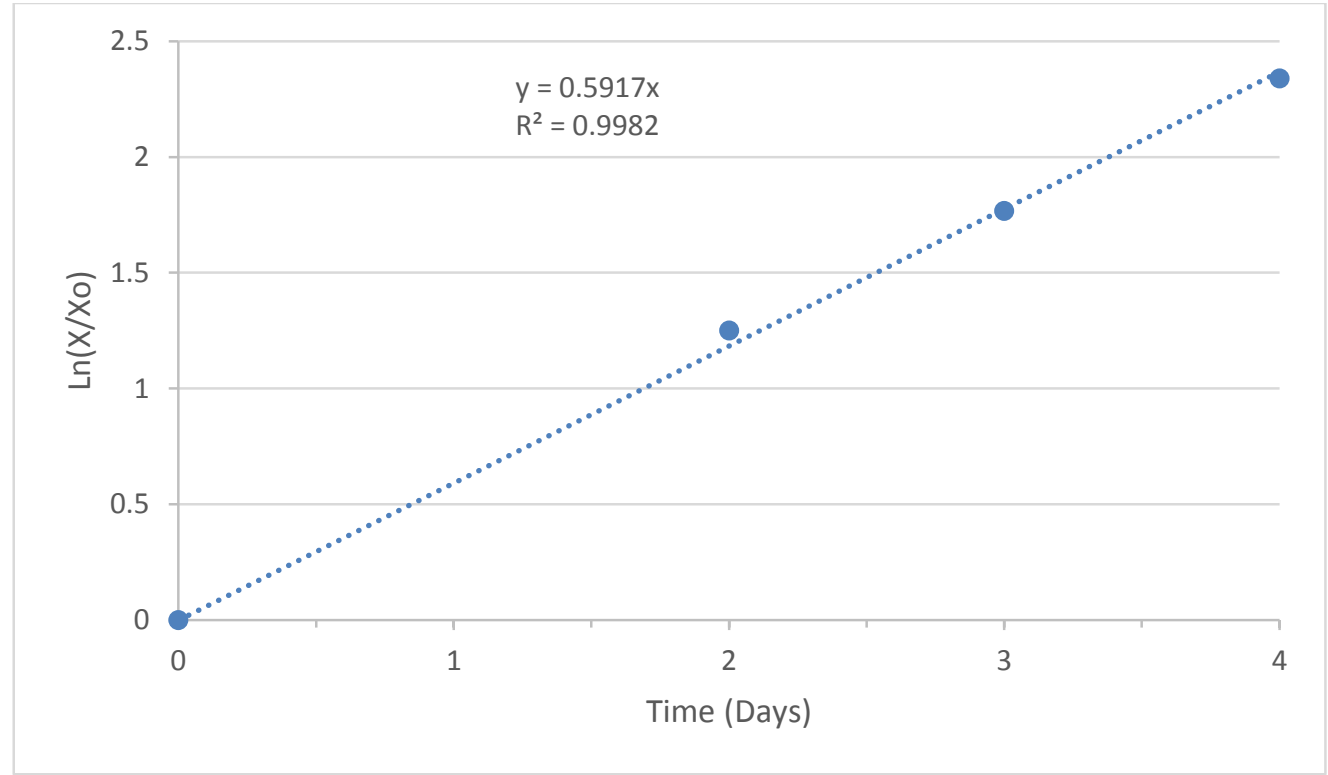

Figure 4.23. Biomass dry weight data from exponential growth phase of Batch Run \#2 as modeled by Equation 4.12

Step 4: Using the value of $\mu_{\text {net }}$ from Figure 4.23 in conjunction with the following equation to calculate biomass productivity published by Zittelli et al. (2013) yielded he results seen in Table 4.6:

$$
P=\mu_{n e t} \Delta X
$$

where $P$ is the biomass productivity as VP, ISP or OAP and $\Delta X$ is the change in biomass dry weight from Day 8 to Day 12 per unit volume of reactor (VP), per square unit of illuminated surface area of reactor photo-stage (ISP), or per square unit of land area (including empty space) occupied by reactor (OAP). Refer to Appendix Q for calculations on biomass productivity. 
Table 4.6. Specifications for the Almeria and prototype PBRs (Acien et al., 2010;

Acien et al., 2012; Dormido et al., 2014)

\begin{tabular}{|c|c|c|c|c|}
\hline \multirow[t]{5}{*}{ Parameter } & \multicolumn{2}{|c|}{ Value } & \multirow[t]{5}{*}{ Unit } & \multirow{2}{*}{$\begin{array}{l}\text { Ratio } \\
\left(\frac{\text { Cal Poly }}{\text { Almeria }}\right)\end{array}$} \\
\hline & Actual & Projected & & \\
\hline & Almeria & Cal Poly & & \\
\hline & PBR & PBR & & \\
\hline & $(3,000 \mathrm{~L})$ & $(3,000 \mathrm{~L})$ & & \\
\hline Dilution rate & $0.34^{\mathrm{a}}$ & $\mathrm{n} / \mathrm{a}$ & $d^{-1}$ & \\
\hline Operation Time & $24^{\mathrm{a}}$ & 24 & hrs d $d^{-1}$ & \\
\hline Air flow rate & $0.1^{\mathrm{a}}$ & 0.35 & $\mathrm{v} / \mathrm{v} / \mathrm{min}$ & $3.5 \mathrm{x}$ \\
\hline Ratio S/V & 14.3 & 20.8 & $\mathrm{~m}^{-1}$ & $1.5 \mathrm{x}$ \\
\hline Mean maximum biomass concentration & $1.26^{\mathrm{a}}$ & 1.0 & $\mathrm{~g} \mathrm{~L}^{-1}$ & $0.8 \mathrm{x}$ \\
\hline Volume-to-photostage-area ratio & 27.3 & $26^{\mathrm{c}}$ & $\mathrm{L} \mathrm{m}^{-2}$ & $1.0 \mathrm{x}$ \\
\hline Volume-to-land-area ratio & 75 & $203^{\mathrm{d}}$ & $\mathrm{L} \mathrm{m}^{-2}$ & $2.7 \mathrm{x}$ \\
\hline Mean volumetric productivity & $0.42^{\mathrm{a}}$ & 0.55 & $\mathrm{~g} \mathrm{~L}^{-1} \mathrm{~d}^{-1}$ & $1.3 \mathrm{x}$ \\
\hline Mean illuminated surface productivity & $11.5^{\mathrm{b}}$ & $14.3^{\mathrm{b}, \mathrm{c}}$ & $\mathrm{g} \mathrm{m}^{-2} \mathrm{~d}^{-1}$ & $1.2 \mathrm{x}$ \\
\hline Mean overall areal productivity & $31.5^{b}$ & $111^{\mathrm{b}, \mathrm{d}}$ & $\mathrm{g} \mathrm{m}^{-2} \mathrm{~d}^{-1}$ & $3.5 \mathrm{x}$ \\
\hline Specific power input required & $11-20^{\mathrm{e}}$ & $8.0-8.7^{\mathrm{e}}$ & $\mathrm{kWh} \mathrm{m}^{-3}$ & $0.4-0.8 \mathrm{x}$ \\
\hline Mean specific biomass productivity & $5.8-10.6^{\mathrm{f}}$ & $17.6-19.1^{\mathrm{f}}$ & $\mathrm{mg} \mathrm{kJ}^{-1}$ & $1.7-3.3 x$ \\
\hline
\end{tabular}

${ }^{a}$ Data collected over a period of one year, from January through December

${ }^{\mathrm{b}}$ Based on volumetric productivity

${ }^{\mathrm{c}}$ Based on calculated dimensions of hypothetic 3,000-L Cal Poly PBR

${ }^{\mathrm{d}}$ Assuming ratio of volume-to-land-area is kept constant during scale up

${ }^{\mathrm{e}}$ For culture circulation and gas exchange only

${ }^{\mathrm{f}}$ Based on specific power input required for circulation and gas exchange

Compared to the Almeria PBR described above, the prototype PBR has a somewhat higher air flow rate and S/V ratio and the volume-to-photostage area ratios for both reactors are very similar (Table 4.3). However, the volume-to-land-area ratio for the $\mathrm{Cal}$ Poly PBR is much greater. The overall areal productivity (OAP) for the Almeria PBR is $31.5 \mathrm{~g} \mathrm{~m}^{-2} \mathrm{~d}^{-1}$, its illuminated surface productivity (ISP) is $11.5 \mathrm{~g} \mathrm{~m}^{-2} \mathrm{~d}^{-1}$ and its volumetric productivity (VP) is $0.42 \mathrm{~g} \mathrm{~L}^{-1} \mathrm{~d}^{-1}$. The data collected for this prototype design suggests an OAP of $111 \mathrm{~g} \mathrm{~m}^{-2} \mathrm{~d}^{-1}$ (350\% that of Almeria), an ISP of $14.3 \mathrm{~g} \mathrm{~m}^{-2} \mathrm{~d}^{-1}(120 \%$ that of Almeria) and a VP of $0.55 \mathrm{~g} \mathrm{~L}^{-1} \mathrm{~d}^{-1}$ (130\% that of Almeria). The relatively high estimated OAP for the Cal Poly PBR is owed to the fact that the Cal Poly prototype PBR 
has a working volume of $235 \mathrm{~L}$ and requires a total land area (including empty space) of $1.16 \mathrm{~m}^{2}$, whereas a 3,000 L PBR unit in the Almeria facility occupies a total land area (including empty space) of $40 \mathrm{~m}^{2}$. Thus, the volume-to-land-area ratio of a 3,000 L unit in the Almeria facility is $75 \mathrm{~L} \mathrm{~m}^{-2}$, whereas for the Cal Poly prototype PBR is $203 \mathrm{~L} \mathrm{~m}^{-2}$. Thus, the volume-to-land-area ratio of the Cal Poly prototype PBR is $271 \%$ that of a 3,000 L PBR unit in Almeria. Refer to Appendix Q for calculations on biomass productivity.

Compared to the Almeria system, the prototype not only excels in OAP but also in terms of energy efficiency. Additional quantification to compare prototype productivity on an energy-input basis was done by dividing volumetric productivity $\left(\mathrm{g} \mathrm{L}^{-1} \mathrm{~d}^{-1}\right)$ by specific power input $\left(\mathrm{kWh} \mathrm{L}^{-1}\right)$ required for culture circulation and gas exchange. Volumetric productivity per Watt per unit volume can be defined as specific biomass productivity (SBP). Specific biomass productivity is a parameter (in $\mathrm{g} \mathrm{W}^{-1} \mathrm{~d}^{-1}$ or $\mathrm{mg} \mathrm{kJ}^{-1}$ ) proposed by Pegallapati et al. (2014) to evaluate PBRs and assess the energy efficiency of a cultivation process. The specific biomass productivity (SBP) of the Almeria PBR ranges between 5.8 and $10.6 \mathrm{mg} \mathrm{kJ}^{-1}$, whereas for the prototype studied it is in the range of 17.6 to $19.1 \mathrm{mg} \mathrm{kJ}^{-1}$ (170 to $330 \%$ that of Almeria). Pegallapati et al. (2014) reported the following values of specific biomass productivity from a literature review: 9.62, 9.98 and $86.7 \mathrm{mg} \mathrm{kJ}^{-1}$ for three outdoor, pilot-scale, flat-plate PBRs; 8.95, 9.55 and $11.9 \mathrm{mg} \mathrm{kJ}^{-1}$ for three pilot-scale, outdoor, bubble-column PBR; $11.7 \mathrm{mg} \mathrm{kJ}^{-1}$ for one pilot-scale, outdoor, helical airlift-driven PBR; 4.27 and $16.5 \mathrm{mg} \mathrm{kJ}^{-1}$ for two indoor, pilot-scale, internally-illuminated bubble columns; 79.4, 88.5 and $124 \mathrm{mg} \mathrm{kJ}^{-1}$ for three outdoor, pilot-scale, internally-illuminated bubble columns. Pegallapati et al. (2014) performed a 
literature review of pilot-scale PBR experiments that contain all parameters necessary to complete an energetic evaluation and asses the energetic performance of some PBRs. Pegallapati et al. (2014) argues that volumetric biomass productivity $\left(\mathrm{g} \mathrm{L}^{-1} \mathrm{~d}^{-1}\right)$ per energy input $\left(\mathrm{W} \mathrm{L}^{-1}\right)$ for cultivation includes that required for culture circulation, gas exchange, heating, cooling and illumination, but energy input due to illumination is not a concern when growing algae outdoors, and energy required for heating or cooling is negligible compared to the energy required for culture circulation and gas exchange (Hulatt \& Thomas, 2011; Pegallapati et al., 2014).

It should be noted that the Almeria PBR runs continuously by dilution, its whole process of growing and harvesting the algae is automated with computer programs, and the dimensions and location of the reactor have been optimized for maximum productivity. Also, data on biomass production for the Almeria PBR has been gathered and studied year round, thus, calculations and operation also account for seasonal variations. The prototype dimensions and its location haven't been optimized. Moreover, data collected on this prototype PBR comes from short-term studies. Short-term studies do not account for seasonal variations of solar irradiance in the environment surrounding the PBR when growing algae outdoors. Moreover, edge or peripheral effects were not taken into account in the calculations. Thus, the biomass productivity values for the Cal Poly prototype PBR listed in Table 4.6 may be overestimates.

A batch operation adds a lag phase to the algae growth, but it can be eliminated if the reactor runs in a continuous mode. In a continuous operation the system is in a steady state, where cells, product and substrate concentration remain constant (Shuler \& Kargi, 2002). In steady state operation the cells can be maintained at their exponential growth 
phase or balanced growth phase, in which all components of the cell grow at the same rate and average cell composition remain approximately constant (Shuler \& Kargi, 2002). In this steady state the cells are being removed at a rate equal to their growth rate, and the growth rate is equal to the dilution rate (Shuler \& Kargi, 2002). An optimization of control variables and harvest by increments are necessary steps to maintain a bioreactor in continuous operation. Since a lag phase decreases biomass productivity, calculations on biomass productivity for the prototype PBR were done ignoring the lag phase seen from inoculation until Day 8 in Figure 4.18.

\subsection{Biofouling in the Prototype PBR}

Biofouling was found to be a significant issue with the materials used to build the prototype PBR and is important to mention since reactor cleaning may be a significant part of maintenance costs. Figure 4.24 depicts the remaining algae in the reactor attached to its walls after draining the culture that had been maintained for a period of 17 days.

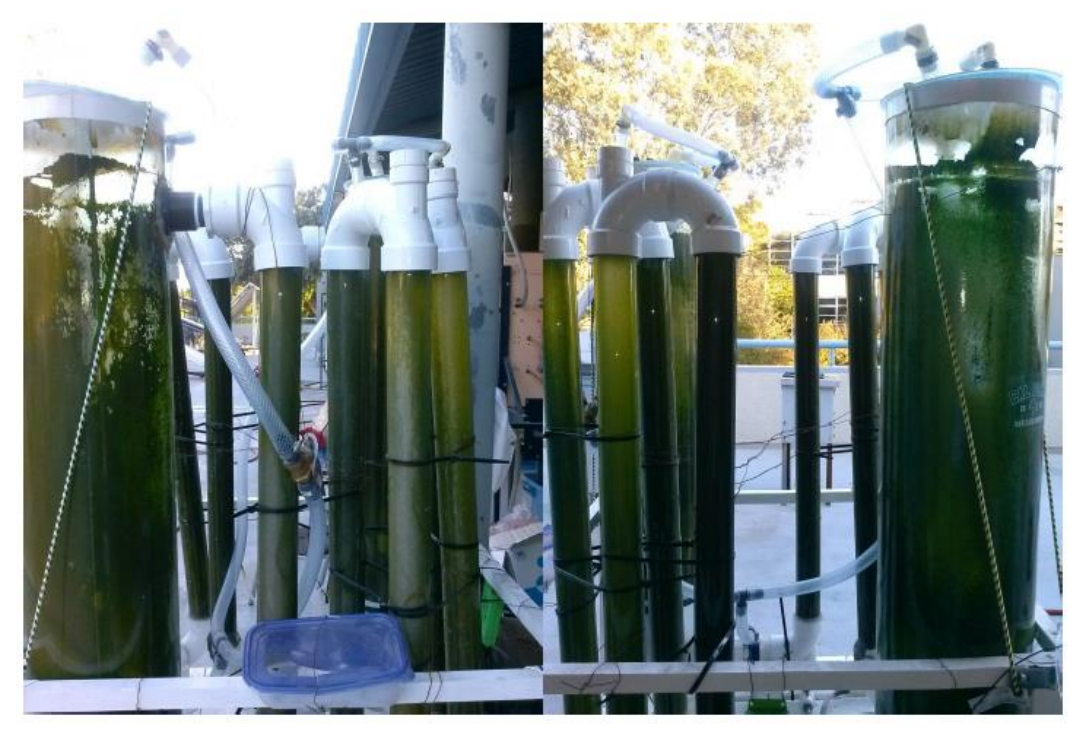

Figure 4.24. Two side views of the reactor showing biofouling of the PBR tubes and columns after draining the culture 
Figure 4.25 shows the reactor walls clean after spending several hours going through the process of cleaning the biofouling in the reactor. It should be noted that this prototype was constructed with relatively inexpensive off-the-shelf materials. Scaling up this proposed PBR design to commercial size merits greater expenditure on construction materials which are easier to clean and resistant to weathering, along with less maintenance requirements. For example, the tubes and columns could be made with polymethylmethacrylate, which is a UV-resistant, rigid and transparent plastic with almost perfect transmission of visible light (Encyclopedia Britannica, 2014). This is the material used for the tubes and columns of the PBR in the Almeria facility (Dormido et al., 2014). Also, the use of a built-in cleaning system that internally cleans the tubes of a PBR without stopping production, such as the one patented by Chaumont et al. (1993) should be considered. This patented system is an agitation system that consists of zerobuoyancy plastic balls that prevent the microalgae from sticking to the reactor walls, and is currently in use by the Almeria PBR (Dormido et al., 2014).

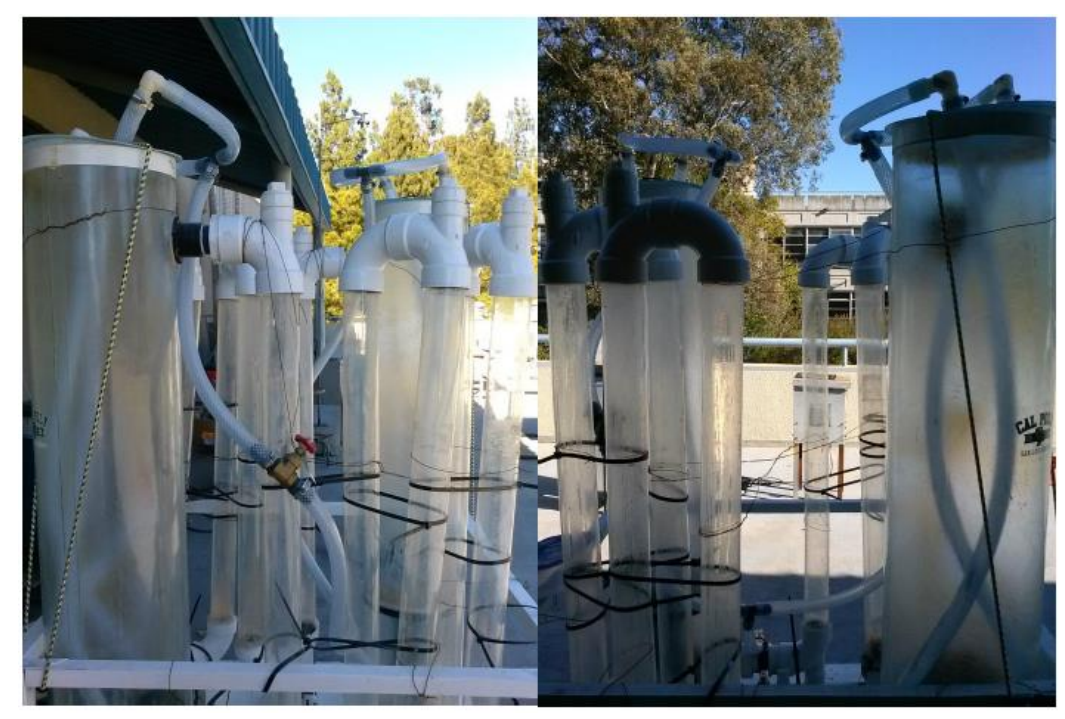

Figure 4.25. Two side views of the reactor after cleaning the biofouling 


\section{CHAPTER V: CONCLUSIONS}

\subsection{Experimental Conclusions}

The data collected for the Cal Poly prototype PBR suggest an average overall areal productivity (OAP) of $111 \mathrm{~g} \mathrm{~m}^{-2} \mathrm{~d}^{-1}$. Data gathered on some companies and universities involved in the microalgae industry at the time of publication of this thesis project report OAPs in the range of 8 to $149 \mathrm{~g} \mathrm{~m}^{-2} \mathrm{~d}^{-1}$ (Table 2.4). Maximum OAPs for short periods under optimum conditions have been reported to be in the range of 30 to $40 \mathrm{~g} \mathrm{~m}^{-2} \mathrm{~d}^{-1}$ for both PBRs and open ponds (Zittelli et al., 2013). Typical OAPs obtained in pilot-scale, outdoors, year-round experiments range between 5 to $12 \mathrm{~g} \mathrm{~m}^{-2} \mathrm{~d}^{-1}$ in PBRs and 5 to $20 \mathrm{~g}$

$\mathrm{m}^{-2} \mathrm{~d}^{-1}$ in open ponds (Zittelli et al., 2013). Zittelli et al. (2013) reports that 12 to $25 \mathrm{~g} \mathrm{~m}^{-2}$ $\mathrm{d}^{-1}$ is feasible, whereas more than that amount is not attainable at large scale with present strains and technologies. It is worth noting that the high values are estimates, while the low values are the actual results of experimentation (Zittelli et al., 2013). Higher values should be considered overestimates unless supported by data from well-organized longterm experiments (Zittelli et al., 2013). Thus, the biomass productivity values for the Cal Poly prototype PBR listed in Table 4.6 may be considered overestimates. Nonetheless, this prototype PBR appears to have a competitive OAP compared to OAPs found in the researched literature.

The specific biomass productivity (SBP) for the Cal Poly prototype PBR is in the range of 17.6 to $19.1 \mathrm{mg} \mathrm{kJ}^{-1}$ with Chlorella vulgaris. Table 5.1 lists SBPs of a few PBRs from a literature review made by Pegallapati et al. (2014). The SBP for the prototype PBR appears to be higher than that of similar designs in the researched literature. 
Table 5.1. Specific biomass productivities of some PBRs (Pegallapati et al., 2014)

\begin{tabular}{|c|c|c|}
\hline Type of PBR & $\begin{array}{l}\text { SBP } \\
\left(\mathrm{mg} \mathrm{kJ}^{-1}\right)\end{array}$ & Algal strain \\
\hline Outdoor, pilot-scale, flat plate & 9.62 & Chlorella sp. \\
\hline Outdoor, pilot-scale, flat plate & 9.98 & Spirulina sp. \\
\hline Outdoor, pilot-scale, flat plate & 86.7 & Nannochloropsis sp. \\
\hline Outdoor, pilot-scale, bubble column & 8.95 & Karlodinium veneficum \\
\hline Outdoor, pilot-scale, bubble column & 9.55 & Alexandrium minutum \\
\hline Outdoor, pilot-scale, bubble-column & 11.9 & Heterosigma akashiwo \\
\hline Outdoor, pilot-scale, airlift-driven helical & 11.7 & Porphyridium cruentum \\
\hline $\begin{array}{l}\text { Indoor, pilot-scale, internally-illuminated } \\
\text { bubble column }\end{array}$ & 4.27 & Nannochloropsis salina \\
\hline $\begin{array}{l}\text { Indoor, pilot-scale, internally-illuminated } \\
\text { bubble column }\end{array}$ & 16.5 & Scenedesmus sp. \\
\hline $\begin{array}{l}\text { Outdoor, pilot-scale, internally-illuminated } \\
\text { bubble column }\end{array}$ & 88.5 & Alexandrium minutum \\
\hline $\begin{array}{l}\text { Outdoor, pilot-scale, internally-illuminated } \\
\text { bubble column }\end{array}$ & 79.4 & Tetraselmis suecica \\
\hline $\begin{array}{l}\text { Outdoor, pilot-scale, internally-illuminated } \\
\text { bubble column }\end{array}$ & 124 & Alexandrium minutum \\
\hline
\end{tabular}

Comparing the Cal Poly prototype PBR with the Almeria system in terms of energy efficiency, we see that the SBP of the Almeria PBR ranges between 5.8 and $10.6 \mathrm{mg} \mathrm{kJ}^{-1}$, whereas for the prototype studied it is in the range of 17.6 to $19.1 \mathrm{mg} \mathrm{kJ}^{-1}$ (170 to $330 \%$ that of Almeria). This may be because the Cal Poly prototype PBR needs 40 to $80 \%$ the total of the specific power input required by the Almeria PBR for culture circulation and gas exchange. However, since SBP is dependent on algal growth, other factors such as growth conditions and algal strain are also likely to influence SBP.

It must be noted that pump efficiency, length of pipe and culture velocity are the most influential variables when determining specific power input from a pump (Geankoplis, 2003). Fraenkel (1986) defines pump efficiency as follows: 


$$
\text { Efficiency }=\frac{\text { hydraulic energy output }}{\text { actual energy input }}
$$

which is always less than one because actual energy needs are greater than the hydraulic energy needs due to inevitable friction losses when transmitting energy. Typical efficiencies of centrifugal pumps range between 50 and $85 \%$ (Geankoplis, 2003). While efficiencies of airlift pumps range between 20 to 30\% (Fraenkel, 1986). For calculations of specific power input, $75 \%$ was assumed for the centrifugal pumps in the Almeria system, whereas $25 \%$ was assumed for the airlift pump in the Cal Poly PBR (Tables 3.5 and 4.3). Also, the tubular stage for both the projected 3,000-L Cal Poly PBR and a 3,000-L PBR in the Almeria facility are similar in length; $440 \mathrm{~m}$ and $400 \mathrm{~m}$ respectively (Tables 3.4 and 4.2). However, the relatively higher SBP for the Cal Poly PBR when compared to the Almeria system may be owed to the fact that the required culture velocity in the tubular stage of the Cal Poly PBR is only $0.2 \mathrm{~m} \mathrm{~s}^{-1}$, whereas the Almeria system uses $0.9 \mathrm{~m} \mathrm{~s}^{-1}$ (Tables 3.4 and 4.2). Since friction losses are proportional to the square of the culture velocity (Equations 3.7 through 3.11 ) the higher flow rate used in the Almeria system would be expected to require much greater pumping energy.

\subsection{Future Research}

Elimination of the centrifugal pump in a tubular PBR design is a concept worth further study for potential energy savings. This may involve the use of lower culture circulation velocities, and therefore further research should be done to determine the effect of culture velocity on overall PBR productivity rates. The higher velocities might also reduce biofouling of PBR surfaces, so this should also be investigated. 
Although fluid dynamics and mass transfer characterization were used to make conclusions worth further study on the proposed PBR design, parameters such as light regime, heat transfer, dilution rate, and shear stress on shear-sensitive microalgae should be studied more in depth with this proposed design for proper operation of the reactor. Studies on solar irradiation as a function of position and location of the reactor could be used to help optimize the light regime on the proposed design. Heat transfer studies could be used to develop systems to keep the reactor at an optimum temperature range with minimum water and energy consumption for heating and cooling. Studies on dilution rate in a long-term experiment could help make stronger conclusions about overall areal productivity and biomass productivity per energy input and eliminate the culture lag phase. Studies of biomass productivity of shear-sensitive microalgae would throw light on the full potential of this prototype design in terms of cultivation efficiencies of this class of algae.

Other aspects of the design, such as structural dimensions, automated control of some variables and biofouling should be improved for scale-up purposes. The number of vertical tubes, spacing between the tubes in the tubular stage and diameter of the tubes and airlift columns should be optimized to maximize culture volume and interception of solar radiation while minimizing yield loses caused by excessive light. Operating variables such as $\mathrm{pH}$, dissolved oxygen, carbon dioxide supply and nutrient concentrations effects on productivity should be studied with an automated computercontrolled system that can acquire data, monitor and control these variables according to the needs of the culture. On the matter of scaling up the proposed design, one approach for scaling up this reactor design would be to keep a ratio of five vertical tubes per airlift 
column (as seen in Figure 3.1) while increasing the height of tubes and columns and keeping diameter constant to achieve the same fluid dynamics and mass transfer behavior seen at the range of aeration rates studied. Another approach to scale up this reactor design would be to increase the length of serpentine tubing while keeping only two airlift columns. These different approaches to scale up could be tested with computer simulation programs for their validation. On the issue of biofouling, addition of a built-in cleaning system that internally cleans the tubes of a PBR without stopping production could be used. Such cleaning systems are currently in common use with tubular PBRs (Dormido et al., 2014). 


\section{REFERENCES}

Acien, F.G., Fernandez, J.M., Gonzalez, C. \& Molina Grima, E. (2010). Microalgae Production Costs. Presentation at AquaFUELs Round Table, Brussels (Belgium), 10-22-10.

Acien, F.G., Fernandez, J.M., Magan, J.J. \& Molina, G.E. (2012). Production cost of a real microalgae production plant and strategies to reduce it. Biotechnology Advances, 30, 1344-1353. DOI: 10.1016/j.biotechadv.2012.02.005.

Albus, J.S., Lacaze, A.D. \& Murphy, K.N. (2010). Open ocean floating algae farm. U.S. Patent Application US2010/4298 A1 (24 June 2010).

American Public Health Association (1995). Standard Methods for the Examination of Water and Wastewater. Washington, DC: American Public Health Association.

Andersen, R.A. (2005). Algal Culturing Techniques. San Diego, CA: Academic Press Inc.

Anderson, D.B. \& Eakin, D.E. (1985). A process for the production of polysaccharides from microalgae. Biotechnol. Bioeng. Symp., 15, 533-47.

Barghbani, R., Rezaei K., \& Javanshir, A. (2012). Investigating the effects of several parameters on the growth of Chlorella vulgaris using Taguchi's experimental approach. International Journal of Biotechnology for Wellness Industries, 1, 128133.

Becker, E.W. (1994). Microalgae: Biotechnology and Microbiology. Cambridge Studies in Biotechnology. Cambridge University Press, Cambridge.

Berzin, I., de Luis, J., Fair, D., Parrish, J., Richtman, K., Chen, F., Polito, B.F., Walker, M., Fowler, B., Lockwood, K. \& Olaizola, M. (2011). Photobioreactor systems positioned on bodies of water. U.S. Patent US 7,980,024 B2 (19 July 2011).

Burbidge, I.M. \& Harper, J.D. (2000). Patent WO 00/12673 (to Addavita Limited).

Bussell, S. (2010). Submersible aquatic algae cultivation system. U.S. Patent Application US2010/0287829 A1 (18 November 2010).

Campbell, M.N. (2008). Biodiesel: algae as a renewable source for liquid fuel. Guelph Engineering Journal, 1, 2-7.

Carvalho, A.P, Meireles, L.A., \& Malcata F.X. (2006). Microalgal Reactors: A Review of Enclosed System Designs and Performances. Biotechnology Progress, 22, 14901506. DOI: 10.1021/bp060065r 
Cathcart, P. (2011). Inexpensive, vertical production photobioreactor. U.S. Patent Application US2011/0027875 A1 (3 February 2011).

Chang, J., Lee, D., Aisyah, R., Yeh, K. \& Chen, C. (2011). Cultivation, photobioreactor design and harvesting of microalgae for biodiesel production: A critical review. Bioresource Technology, 102, 71-81. DOI:10.1016/j.biortech.2010.06.159

Chaumont, D. (1993). Biotechnology of algal biomass production: a review of systems for outdoor mass culture. J. Appl. Phycol., 5, 593-604.

Chaumont, D., Thepenier, C. \& Gudin, C. (1988). Scaling up a tubular photoreactor for continuous culture of Porphyridium cruentum - From laboratory to pilot plant. In: Algal Biotechnology (eds T. Stadler, J. Morillon, M.C. Verdus, W. Karamanos, H. Morvan \& D. Christiaen), pp. 199-208. Elsevier Applied Science, London.

Chaumont, D., Dos Santos, P.F. \& Sauze, L. (1993). Apparatus for the automatic, continuous cleaning of the pipe of the solar receptor of a photobioreactor. U.S. Patent US005242827A (7 September 1993).

Chini Zittelli, G., Lavista, F., Bastianini, A., Rodolfi, L., Vincenzini, M. \& Tredici, M.R. (1999). Production of eicosapentaenoic acid by Nannochloropsis sp. cultures in outdoor tubular photobioreactors. J. Biotech., 70, 299-312.

Chisti, M.Y. (1989). Airlift Bioreactors. London (England): Elsevier Applied Science.

Chisti, M.Y. (2007). Biodiesel from microalgae. Biotechnology Advances, 25, 294-306. DOI: 10.1016/j.biotechadv.2007.02.001

Chisti, Y., Camacho, F. G., Fernandez, F.G.A., \& Grima, E.M. (1999). Photobioreactors: light regime, mass transfer, and scaleup. Journal of Biotechnology, 70, 231-247. PII: S0168-1656(99)00078-4

Chisti, Y., Molina, E., Feranadez, J., \& Acien, F.G., (2001). Tubular photobioreactor design for algal cultures. Journal of Biotechnology, 92, 113-131.

Chojnacka, K. \& Marquez-Rocha, F.J. (2004). Kinetic and stoichiometric relationships of the energy and carbon metabolism in the culture of microalgae. Biotechnology, 3 , 21-34.

Cohen, E. \& Arad (Malis), S. (1989). A closed system for outdoor cultivation of Porphyridium Biomass, 18, 59-67. 
Cook, P.M. (1950). Large-scale culture of Chlorella. In: The Culturing of Algae (eds J. Brunel, G.W. Prescott \& L.H. Tiffany), pp. 53-75. Charles F. Kettering Foundation, Yellow Springs, Ohio.

Danner Manufacturing Inc. (2014). Retrieved from http://www.pondmaster.com/Store/Products/Danner/PID-02515.aspx

Das, D., Kumar, K., Dasgupta, C.N., Nayak, B., \& Linblad, P. (2011). Development of suitable photobioreactors for $\mathrm{CO}_{2}$ sequestration addressing global warming using green algae and cyanobacteria. Bioresource Technology, 102: 4945-4953.

Davis, R., Aden, A., \& Pienkos, P.T. (2011). Techno-economic analysis of autotrophic microalgae for fuel production. Applied Energy, 88, 3524-3531. DOI:10.1016/j.apenergy.2011.04.018

Davis, E.A., Dedrick, J., French, C.S., Milner, H.W., Myers, J., Smith, J.H.C. \& Spoehr, H.A. (1953). Laboratory experiments on Chlorella culture at the Carnegie Institution of Washington, Department of Plant Biology. In: Algal Culture from Laboratory to Pilot Plant (ed. J.S. Burlew), pp. 105-53. Carnegie Institution of Washington Publication No. 600, Carnegie Institution, Washington, D.C.

Dodd, J.C. (1986). Elements of pond design and construction. In: Handbook of Microalgal Mass Cultures (ed. A. Richmond), pp. 265-84. CRC Press, Inc., Boca Raton, Florida.

Dormido, R., Sanchez, J., Duro, N., Dormido-Canto, S., Guinaldo, M. \& Dormido, S. (2014). An Interactive Tool for Outdoor Computer Controlled Cultivation of Microalgae in a Tubular Photobioreactor System. Sensors, 14: 4466-4483.

Encyclopedia Britannica (2014). Retrieved from http://www.britannica.com/

Fargione, J., Hill, J., Tilman, D., Polasky, S. \& Hawthorne, P. (2008). Land clearing and the biofuel carbon debt. Science, 319, 1235-1238. DOI: 10.1126/science.1152747

Fernandez-Sevilla, J.M., Acien Fernandez, F.G. \& Molina Grima, E. (2010). Biotechnological production of lutein and its applications. Appl. Microbiol. Biotechnol.86: 27-40.

FlexAir (2014). Retrieved from https://pentairaes.com/index.php/flexair-9-discdiffuser.html

Fraenkel, P.L. (1986). Water Lifting Devices. Rome, Italy: Food and Agriculture Organization of the United Nations. Retrieved from http://www.fao.org/docrep/010/ah810e/ah810e00.htm\# 
Garcia Camacho, F., Contreraz Gomez, A., Acien Fernandez, F.G., Fernandez Sevilla, J. $\&$ Molina Grima, E. (1999). Use of concentric-tube airlift photobioreactors for microalgal outdoor mass cultures. Enzyme Microb. Technol., 24, 165-72.

Geankoplis, C. J. (2003). Transport Processes and Separation Process Principles. Upper Saddle River, NJ: Pearson Education Inc.

Green, F.B., Lundquist, T.J. \& Oswald, W.J., (1995). Energetics of advanced integrated wastewater pond systems, Water Science and Technology, 31, 12, 9-20.

Hirabayashi, S. (1999). Patent WO 99/50384 (to Micro Gaia Co. Ltd).

Hirabayashi, S. (2001). Patent WO 01/23519 (to Micro Gaia Co. Ltd).

Hu, Q., Guterman, H. \& Richmond, A. (1996). A flat inclined modular photobioreactor (FIMP) for outdoor mass cultivation of photoautotrophs. Biotechnol. Bioeng., 51, $51-60$.

Hu, Q. \& Richmond, A. (1994). Optimizing the population density in Isochrysis galbana grown outdoors in a glass column photobioreactor. J. Appl. Phycol., 6, 391-96.

Huang, G.H., Chen, F., Wei, D., Zhang, X.W. \& Chen, G. (2010). Biodiesel production by microalgal biotechnology. Applied Energy, 87, 38-46.

Hulatt, J.C. \& Thomas, D. N. (2011). Energy efficiency of an outdoor microalgal photobioreactor sited at mid-temperate latitude. Bioresource Technology, 102, 6687-6695. DOI:10.1016/j.biortech.2011.03.098.

Huntley, M.E. \& Redalje, D.G. (2006). CO2 mitigation and renewable oil from photosynthetic microbes: a new appraisal. Mitigat. Adapt. Strat. Global Change 12: $573-608$.

Janssen, M., Tramper, J., Mur, L.R., \& Wijffels, R.H. (2002). Enclosed Outdoor Photobioreactors: Light Regime, Photosynthetic Efficiency, Scale-Up, and Future Prospects. Biotechnology and Bioengineering, 81, 193-210. DOI: 10.1002/bit.10468

Jensen, G. S., Ginsberg, D. I., and Drapeau, M. S. (2001). Bluegreen algae as an immuno-enhancer and biomodulator. J. Am. Nutraceutical Association, 3, 24-30.

Jorquera, O., Kiperstok A., Sales, E.A., Embirucu, M., \& Ghirardi M.L. (2010). Comparative energy life-cycle analyses of microalgal biomass production in open ponds and photobioreactors. Bioresource Technology, 101, 1406-1413.

Juttner, F. (1982). Mass cultivation of microalgae and phototrophic bacteria under sterile conditions. Proc. Biochem., 17, 2-7. 
Key Instruments (2014). Retrieved from http://www.keyinstruments.com/

Kruger, G.H.J. \& Eloff, J.N. (1981). Defined algal production systems for the culture of microalgae. In: Wastewater for Aquaculture (eds J.U. Grobbelaar, C.J. Soeder \& D.F. Toerien), pp. 16-23. University of Orange Free State Publication, Bloemfontein, South Africa.

Lee, Y.K. (1986). Enclosed bioreactors for the mass cultivation of photosynthetic microorganisms: the future trend. Trends in Biotechnology, 4, 186-89.

Lee, Y.K. (2001). Microalgal mass culture systems and methods: Their limitation and potential. J. Appl. Phycol., 13, 307-15.

Letzel et al. (1999). Gas holdup and mass transfer in bubble column reactors operated at elevated pressure. Chem. Eng. Sci., 54: 2237-2246.

Licamele, J.D. \& White, C.L. (2011). V-trough photobioreactor system and method of use. U.S. Patent Application US2011/0258920 A1 (27 October 2011).

Mata, T.M., Martins, A.A. \& Caetano, N.S. (2010). Microalgae for biodiesel production and other applications: a review. Renew. Sust. Energ. Rev., 14, 217-232.

Mehlitz, T.H. (2009). Temperature Influence and Heat Management Requirements of Microalgae Cultivation in Photobioreactors, M.S. thesis, BioResource and Agricultural Engineering Department, California Polytechnic State University, San Luis Obispo.

Merchuk, J.C., \& Gluz, M. (2003). Bioreactors, Air-lift Reactors. Encyclopedia of Bioprocess Technology. 320-352. DOI: 10.1002/0471250589.ebt029

Milner, H.W. (1953). Rocking tray. In: Algal Culture from Laboratory to Pilot Plant (ed. J.S. Burlew), p. 108. Carnegie Institution of Washington Publication No. 600, Carnegie Institution, Washington, D.C.

Milwaukee (2014). Retrieved from http://www.milwaukeetesters.com/SMS120.html

Miyamoto, K., Wable, O. \& Benemann, J.R. (1988) Vertical tubular reactor for microalgae cultivation. Biotechnol. Lett., 10, 703-708.

Molina Grima, E. (1999). Microalgae, mass culture methods. In: Encyclopedia of Bioprocess Technology: Fermentation, Biocatalysis and Bioseparation (eds M.C. Flickinger \& S.W. Drew), pp. 1753-69. J. Wiley \& Sons, New York.

Molina Grima, E., Acien Fernandez, F.G., Garcia Camacho, F., Camacho Rubio, F. \& Chisti, Y. (2000). Scale-up of tubular photobioreactors. Journal of Applied Phycology, 12, 355-368. 
Molina Grima, E., Fernandez, J.M., Acien, F.G., Sanchez, J.F., Garcia, J., Magan, J.J. \& Perez, J. (2006). Production of lutein from the microalga Scenedesmus almeriensis in an industrial size photobioreactor: case study. Publicaciones de Cajamar Caja Rural. Retrieved on October 10, 2014. Retrieved from http://www.publicacionescajamar.es/ series-tematicas/centros-experimentales-laspalmerillas/production-of-lutein-from-the-microalga-scenedesmus-almeriensis-inan-industrial-size-photobioreactor-case/

Molina Grima, E., Sierra, E., Acien, J.M., Fernendez, J.L., \& Gonzalez G.C. (2008). Characterization of a flat plate photobioreactor for the production of microalgae. Chemical Engineering Journal, 138, 136-147. DOI:10.1016/j.cej.2007.06.004

Mortimer, J., Thomas, S. \& Temekenidis, P.A. (1999). Patent WO 99/46360 (to Applied Photosynthetics Limited).

Mortimer, J. (2000). Patent WO 00/05337 (to Biosynthesis Limited).

Mottahedeh, S. \& Tredici, M.R. (2012). Low cost integrated pond-photobioreactor. Canadian Patent Application 2,764,291 (16 January 2012).

Moutafchieva, D., Popova, D., Dimitrova, M., \& Tchaoushev, S. (2013). Experimental determination of the volumetric mass transfer coefficient. Journal of Chemical Technology and Metallurgy, 48, 4: 351-356.

Muller Feuga, A. \& Lemar, M. (2011). Reaction jacket for a photosynthetic reactor and related photosynthetic reactor. U.S. Patent Application US2011/0111484 A1 (12 May 2011).

National Research Council Canada. (2013). Retrieved from http://archive.nrccnrc.gc.ca/eng/facilities/imb/marine-research.html.

Nitto-Kohki Co. (2014). Retrieved from http://www.nitto-kohki.eu/en/productsen/pumps-a-compressors/air-blowers/item/la-120a.html

Norsker, N. H., Barbosa, M. J., Vermue, M. H., \& Wijffels, R.H. (2012). On energy balance and production costs in tubular and flat panel photobioreactors. Technikfolgenabschätzung - Theorie und Praxis, 21, 1: 54-62.

Oilgae (2013). Energy from Algae: Products, Market, Processes \& Strategies. Comprehensive Report. Retrieved from http://www.oilgae.com/ref/report/oilgae_ reports.html

Olaizola, M. (2000). Commercial production of astaxanthin from Haematococcus pluvialis using 25,000-liter outdoor photobioreactors. J. Appl. Phycol., 12, 499506. 
Olaizola, M. (2003). Commercial development of microalgal biotechnology: from the test tube to the marketplace. Biomolecular Engineering, 20, 459-466. DOI: 10.1016/S1389-0344(03)00076-5

Oswald, W.J. (1988). Large-scale algal culture systems (engineering aspects). In: Microalgal Biotechnology (eds M.A. Borowitzka \& L.J. Borowitzka), pp. 357-94. Cambridge University Press, Cambridge.

Patel, M.H., Brennan, F. \& Magan, N. (2011). Biomass farming system and method. U.S. Patent Application US2011/0283608 A1 (24 November 2011).

Pegallapati, A.K., Arudchelvam, Y. \& Nirmalakhandan, N. (2014). Energetic performance of photobioreactors for algal cultivation. Environmental Science and Technology Letters, 1, 2-7.

Pessarakli, M. (1999). Handbook of Plant and Crop Stress. New York, NY: Marcel Dekker Inc.

Pirt, S.J. (1983). Patent UK 2,118,572 (to Queen Elizabeth College, University of London).

Pirt, S.J., Lee, Y.K., Walach, M.R., Pirt, M.W., Balyuzi, H.H. \& Bazin, M.J. (1983). A tubular bioreactor for photosynthetic production from carbon dioxide: design and performances. J. Chem. Tech. Biotechnol., 33B, 35-58.

Prokop, A. \& Erickson, L.E. (1995). Photobioreactors. In: Bioreactor System Design (eds J.A. Asenjo \& J.C. Merchuk), pp. 441-77. Marcel Dekker, Inc., New York.

Pohl, P., Kohlhase, M., \& Martin, M. (1988). Photobioreactors for the axenic mass cultivation of microalgae. In: Algal Biotechnology; Stadler, T., Mollion, J., Verdus, M.-C., Karamanos, Y., Morvan, H., Christiaen, D., Eds.; Elsevier: New York: pp 209-218.

Pulz, O. (2001). Photobioreactors: production systems for phototrophic microorganisms. Appl. Microb. Biotechnol., 57, 287-93.

Pulz, O. \& Scheibenbogen, K. (1998). Photobioreactors: design and performance with respect to light energy input. In: Advances in Biochemical Engineering/Biotechnology (ed. T. Scheper), pp. 123-52. Springer-Verlag, Berlin.

Rachlin, J. W., \& Grosso, A. (1991). The effects of $\mathrm{pH}$ on the growth of Chlorella vulgaris and its interactions with cadmium toxicity. Arch. Environ. Contam. Toxicol, 20: 505-508.

Raico Engineers Inc. (2014). Retrieved from http://www.blowersandblowersindia.com /ring-blowers.php 
Ramos de Ortega, A. \& Roux, J.C. (1986). Production of Chlorella biomass in different types of flat bioreactors in temperate zones. Biomass, 10, 141-56.

Richmond, A. (1999). Physiological principles and modes of cultivation in mass production of photoautotrophic microalgae. In: Chemicals from Microalgae (ed. Z. Cohen), pp. 353-86. Taylor \& Francis, London.

Richmond, A. \& Becker, E.W. (1986). Technological aspects of mass cultivation, a general outline. In: CRC Handbook of MicroalgalMass Culture (ed. A. Richmond), pp. 245-63. CRC Press, Inc., Boca Raton, Florida.

Richmond, A., Boussiba, S., Vonshak, A. \& Kopel, R. (1993). A new tubular reactor for mass production of microalgae outdoors. J. Appl. Phycol., 5, 327-32. Lee, Y.K., Ding, S.Y., Low, C.S., Chang, Y.C., Forday, W.L. \& Chew, P.C. (1995) Design and performance of an a-type tubular photobioreactor for mass cultivation of microalgae. J. Appl. Phycol., 7, 47-51.

Richmond, A. \& Zhang, Z. (2001). Optimization of a flat plate glass reactor for mass production of Nannochloropsis sp. outdoors. J. Biotechnol., 85, 259-69.

Robinson, L.F. \& Morrison, A.W. (1987). Patent EP 0239272 B1 (to Biotechna Limited).

Rodolfi, L., Chini Zittelli, G., Bassi, N., Padovani, G., Biondi, N., Bonini, G. \& Tredici, M.R. (2008). Microalgae for oil: strain selection, induction of lipid synthesis and outdoor mass cultivation in a low-cost photobioreactor. Biotechnol. Bioeng. 102: $100-112$.

Samson, R. \& LeDuy, A. (1985). Multistage continuous cultivation of blue-green alga Spirulina maxima in the flat tank photobioreactors with recycle. Can. J. Chem. Eng., 63, 105-12.

Sanchez, J.F., Fernandez-Sevilla, J.M., Acien, F.G., Ceron, M.C., Perez-Parra, J. \& Molina-Grima, E. (2008). Biomass and lutein productivity of Scenedesmus almeriensis: influence of irradiance, dilution rate and temperature. Appl. Microbiol. Biotechnol., 79, 719-729. DOI 10.1007/s00253-008-1494-2.

Setlik, I., Komarek, J. \& Prokes, B. (1967). Short account of the activities from 1960 to 1965. In: Annual Report of the Laboratory of Experimental Algology and Department of Applied Algology for the year 1966 (ed. J. Necas \& O. Lhotsky), pp. 5-36. Knihtisk, Praha, Czechoslovakia.

Sheehan, J., Dunahay, T., Benemann, J., Roessler, P., (1998). A Look Back at the U.S. Department of Energy's Aquatic Species Program-Biodiesel from Algae. National Renewable Energy Program. 
Shuler, M.L., \& Kargi F. (2002). Bioprocess Engineering: Basic Concepts. Upper Sadle River, NJ: Pearson Education Inc.

Sierra, E., Acien, F.G., Fernandez, J.M., Garcia, J.L., Gonzales, C. \& Molina, E. (2008). Characterization of a flat plate photobioreactor for the production of microalgae. Chem. Eng. J. 138: 136-147.

Spolaore, P., Joannis-Cassan, C., Duran, E., \& Isambert, A. (2006). Commercial Applications of Microalgae. Journal of Bioscience and Bioengineering, 101, 8797. DOI: $10.1263 / \mathrm{jbb} .101 .87$

Tamiya, H., Hase, E., Shibata, K., Mituya, A., Iwamura, T., Nihei, T. \& Sasa, T. (1953). Kinetics of growth of Chlorella, with special reference to its dependence on quantity of available light and temperature. In: Algal Culture from Laboratory to Pilot Plant (ed. J.S. Burlew), pp. 204-32. Carnegie Institution of Washington Publication No. 600, Carnegie Institution, Washington, D.C.

Torzillo, G. (1997). Tubular Bioreactors. In: Spirulina platensis (Arthrospira): Physiology, Cell-biology and Biotechnology (ed. A. Vonshak), pp. 101-15. Taylor \& Francis, London.

Torzillo, G., Carlozzi, P., Pushparaj, B., Montaini, E. \& Materassi, R. (1993). A twoplane tubular photobioreactor for outdoor culture of Spirulina. Biotechnol. Bioeng., 42, 891-98.

Torzillo, G., Pushparaj, B., Bocci, F., Balloni, W., Materassi, R. \& Florenzano, G. (1986). Production of Spirulina biomass in closed photobioreactors. Biomass, 11, $61-74$.

Travesio, L., Hall, D.O., Rao, K.K., Benitez, F., Sanchez, E. \& Borja, R. (2001). A helical tubular reactor producing Spirulina in a semicontinuous mode. Int. Biodeterg. Biodegrad, 47, 151-155.

Tredici, M.R. \& Chini Zittelli, G. (1997). Cultivation of Spirulina (Arthrospira) platensis in flat plate reactors. In: Spirulina platensis (Arthrospira): Physiology, Cellbiology and Biotechnology (ed. A. Vonshak), pp. 117-30. Taylor \& Francis, London.

Tredici, M.R. (1999). Photobioreactors. In: Encyclopedia of Bioprocess Technology: Fermentation, Biocatalysis and Bioseparation (ed. M.C. Flickinger \& S.W. Drew), pp. 395-419. J. Wiley \& Sons, New York.

Tredici, M.R. (2004). Mass production of microalgae: Photobioreactors. In: Richmond A., editor. Handbook of microalgal culture: biotechnology and applied phycology. Oxford: Blackwell Publishing, p 178-214. 
Tredici, M.R., Carlozzi, P., Chini Zittelli, G. \& Materassi, R. (1991). A vertical alveolar panel (VAP) for outdoor mass cultivation of microalgae and cyanobacteria.

Bioresour. Technol., 38, 153-59.

Tredici, M.R. \& Chini Zittelli, G. (1997). Cultivation of Spirulina (Arthrospira) platensis in flat plate reactors. In: Spirulina platensis (Arthrospira): Physiology, Cellbiology and Biotechnology (ed. A. Vonshak), pp. 117-30. Taylor \& Francis, London.

Tredici, M.R. \& Chini Zittelli, G. (1998). Efficiency of sunlight utilization: tubular versus flat photobioreactors. Biotechnol. Bioeng., 57, 187-97.

Tredici, M.R. \& Materassi, R. (1992). From open ponds to vertical alveolar panels: the Italian experience in the development of reactors for the mass cultivation of phototrophic microorganisms. J. Appl. Phycol., 4, 221-31.

Ugwu, C.U., Aoyagi, H., \& Uchiyama, H. (2008). Photobioreactors for mass cultivation of algae. Bioresource Technology, 99, 4021-4028. DOI:

10.1016/j.biortech.2007.01.046

University of Texas (2014). Bristol Medium: Media Detail. Retrieved from http://web.biosci.utexas.edu/utex/mediaDetail.aspx?mediaID=29

Vernier Software \& Technology (2014). Retrieved from http://www.vernier.com/

ViaAqua (2014). Retrieved from http://www.petmountain.com/brands/84/1/via-aqua.html

Viagrow (2014). Retrieved from http://www.amazon.com/Viagrow-Micro-Air-StoneDiffuser/dp/B00CF496VI

Weissman, J.C. \& Goebel, R.P. (1988). Photobioreactor design: mixing, carbon utilization, and oxygen accumulation. Biotechnology and Bioengineering, 31, 336-344.

Xu, L., Weathers, P.J., Xiong, X.R., \& Liu, C.Z. (2009). Microalgal bioreactors: Challenges and opportunities. Eng. Life Sci. 2009, 9, 3, 178-189. DOI: 10.1002/elsc.20080011

Zittelli, G.C., Biondi, N., Rodolfi, L., \& Tredici, M.R. (2013). Photobioreactors for mass production of microalgae. In: Richmond, A. \& Hu, Q., editors. Handbook of microalgal culture: applied phycology and biotechnology. Oxford: Blackwell Publishing, p 225-266. 


\section{APPENDIX A: TOTAL SUSPENDED SOLIDS DATA}

\begin{tabular}{|c|c|c|c|c|c|c|c|c|}
\hline Time & Date & Day \# & $\begin{array}{l}\text { Tray } \\
\text { Code }\end{array}$ & $\begin{array}{c}\text { Tray + } \\
\text { Filter } \\
\text { Tare } \\
\text { (g) }\end{array}$ & $\begin{array}{c}\text { Sample } \\
\text { Volume } \\
(\mathbf{m L})\end{array}$ & $\begin{array}{c}\text { Tray + } \\
\text { Filter } \\
\text { at } 105{ }^{\circ} \mathrm{C} \\
\text { (g) }\end{array}$ & $\begin{array}{c}\text { TSS } \\
(\mathrm{g} / \mathrm{L})\end{array}$ & $\begin{array}{c}\text { TSS } \\
\text { Average } \\
\text { (g/L) }\end{array}$ \\
\hline $4 \mathrm{pm}$ & $5 / 29 / 2014$ & 0 & 1 & 1.07142 & 100 & 1.08257 & 0.1115 & \\
\hline $4 \mathrm{pm}$ & $5 / 29 / 2014$ & 0 & 2 & 1.0778 & 100 & 1.09053 & 0.1273 & 0.1182 \\
\hline $4 \mathrm{pm}$ & $5 / 29 / 2014$ & 0 & 3 & 1.08234 & 100 & 1.09392 & 0.1158 & \\
\hline $10 \mathrm{pm}$ & $5 / 30 / 2014$ & 1 & 1 & 1.24666 & 30 & 1.25026 & 0.1200 & \\
\hline $10 \mathrm{pm}$ & $5 / 30 / 2014$ & 1 & 2 & 1.26774 & 30 & 1.27034 & 0.0867 & 0.1200 \\
\hline $10 \mathrm{pm}$ & $5 / 30 / 2014$ & 1 & 3 & 1.25335 & 30 & 1.25795 & 0.1533 & \\
\hline $10 \mathrm{pm}$ & $6 / 1 / 2014$ & 3 & 1 & 1.25247 & 30 & 1.25547 & 0.1000 & \\
\hline $10 \mathrm{pm}$ & $6 / 1 / 2014$ & 3 & 2 & 1.25068 & 30 & 1.25378 & 0.1033 & 0.1089 \\
\hline $10 \mathrm{pm}$ & $6 / 1 / 2014$ & 3 & 3 & 1.26006 & 30 & 1.26376 & 0.1233 & \\
\hline $10 \mathrm{pm}$ & $6 / 4 / 2014$ & 6 & 1 & 1.26556 & 30 & 1.26936 & 0.1267 & \\
\hline $10 \mathrm{pm}$ & $6 / 4 / 2014$ & 6 & 2 & 1.25409 & 30 & 1.25829 & 0.14 & 0.1211 \\
\hline $10 \mathrm{pm}$ & $6 / 4 / 2014$ & 6 & 3 & 1.25706 & 30 & 1.25996 & 0.0967 & \\
\hline $10 \mathrm{pm}$ & 6/6/2014 & 8 & 1 & 1.27001 & 30 & 1.27311 & 0.1033 & \\
\hline $10 \mathrm{pm}$ & $6 / 6 / 2014$ & 8 & 2 & 1.2582 & 30 & 1.262 & 0.1267 & 0.1 \\
\hline $10 \mathrm{pm}$ & 6/6/2014 & 8 & 3 & 1.25584 & 30 & 1.25794 & 0.07 & \\
\hline $10 \mathrm{pm}$ & 6/8/2014 & 10 & 1 & 1.25499 & 50 & 1.26959 & 0.292 & \\
\hline $10 \mathrm{pm}$ & 6/8/2014 & 10 & 2 & 1.25598 & 50 & 1.27058 & 0.292 & 0.3493 \\
\hline $10 \mathrm{pm}$ & $6 / 8 / 2014$ & 10 & 3 & 1.2508 & 50 & 1.274 & 0.464 & \\
\hline $10 \mathrm{pm}$ & $6 / 9 / 2014$ & 11 & 1 & 1.26206 & 50 & 1.29046 & 0.568 & \\
\hline $10 \mathrm{pm}$ & 6/9/2014 & 11 & 2 & 1.25482 & 50 & 1.28082 & 0.52 & 0.5853 \\
\hline $10 \mathrm{pm}$ & 6/9/2014 & 11 & 3 & 1.25172 & 50 & 1.28512 & 0.668 & \\
\hline
\end{tabular}




\begin{tabular}{|c|c|c|c|c|c|c|c|c|}
\hline Time & Date & Day \# & $\begin{array}{l}\text { Tray } \\
\text { Code }\end{array}$ & $\begin{array}{c}\text { Tray + } \\
\text { Filter } \\
\text { Tare } \\
\text { (g) }\end{array}$ & $\begin{array}{c}\text { Sample } \\
\text { Volume } \\
(\mathbf{m L})\end{array}$ & $\begin{array}{c}\text { Tray + } \\
\text { Filter } \\
\text { at } 105{ }^{\circ} \mathrm{C} \\
\quad(\mathrm{g})\end{array}$ & $\begin{array}{c}\text { TSS } \\
(\mathrm{g} / \mathrm{L})\end{array}$ & $\begin{array}{c}\text { TSS } \\
\text { Average } \\
(\mathrm{g} / \mathrm{L})\end{array}$ \\
\hline $10 \mathrm{pm}$ & $6 / 10 / 2014$ & 12 & 1 & 1.25674 & 100 & 1.36344 & 1.067 & \\
\hline 10pm & $6 / 10 / 2014$ & 12 & 2 & 1.25972 & 100 & 1.36702 & 1.073 & 1.0373 \\
\hline $10 \mathrm{pm}$ & $6 / 10 / 2014$ & 12 & 3 & 1.2587 & 100 & 1.3559 & 0.972 & \\
\hline $10 \mathrm{pm}$ & $6 / 11 / 2014$ & 13 & 1 & 1.25767 & 100 & 1.36197 & 1.043 & \\
\hline $10 \mathrm{pm}$ & $6 / 11 / 2014$ & 13 & 2 & 1.25465 & 100 & 1.34895 & 0.943 & 0.985 \\
\hline $10 \mathrm{pm}$ & $6 / 11 / 2014$ & 13 & 3 & 1.25621 & 100 & 1.35311 & 0.969 & \\
\hline $10 \mathrm{pm}$ & $6 / 12 / 2014$ & 14 & 1 & 1.24772 & 100 & 1.35732 & 1.096 & \\
\hline 10pm & $6 / 12 / 2014$ & 14 & 2 & 1.2524 & 100 & 1.3626 & 1.102 & 1.104 \\
\hline $10 \mathrm{pm}$ & $6 / 12 / 2014$ & 14 & 3 & 1.25514 & 100 & 1.36654 & 1.114 & \\
\hline $10 \mathrm{pm}$ & $6 / 13 / 2014$ & 15 & 1 & 1.25632 & 100 & 1.31962 & 0.633 & \\
\hline $10 \mathrm{pm}$ & $6 / 13 / 2014$ & 15 & 2 & 1.25981 & 100 & 1.33871 & 0.789 & 0.6873 \\
\hline $10 \mathrm{pm}$ & $6 / 13 / 2014$ & 15 & 3 & 1.24982 & 100 & 1.31382 & 0.64 & \\
\hline $10 \mathrm{pm}$ & $6 / 14 / 2014$ & 16 & 1 & 1.25778 & 100 & 1.30498 & 0.472 & \\
\hline $10 \mathrm{pm}$ & $6 / 14 / 2014$ & 16 & 2 & 1.25974 & 100 & 1.30904 & 0.493 & 0.4973 \\
\hline $10 \mathrm{pm}$ & $6 / 14 / 2014$ & 16 & 3 & 1.25281 & 100 & 1.30551 & 0.527 & \\
\hline $10 \mathrm{pm}$ & $6 / 15 / 2014$ & 17 & 1 & 1.25463 & 50 & 1.27849 & 0.4772 & \\
\hline 10pm & $6 / 15 / 2014$ & 17 & 2 & 1.25382 & 50 & 1.27841 & 0.4918 & 0.4953 \\
\hline $10 \mathrm{pm}$ & $6 / 15 / 2014$ & 17 & 3 & 1.25623 & 50 & 1.28208 & 0.517 & \\
\hline
\end{tabular}




\section{APPENDIX B: N-NO ${ }_{3}$ CONCENTRATION DATA}

\begin{tabular}{|c|c|c|c|c|}
\hline Date & Time & Time & NO3-N & NO3-N \\
\hline & (Days) & & (mg/L) & (\% remaining) \\
\hline $5 / 29 / 2014$ & 0 & 4pm & 69.4 & 99.1 \\
\hline $5 / 30 / 2014$ & 1 & 10pm & 60.8 & 86.9 \\
\hline $6 / 1 / 2014$ & 3 & 10pm & 65 & 92.9 \\
\hline $6 / 4 / 2014$ & 6 & 10pm & 59.4 & 84.9 \\
\hline $6 / 6 / 2014$ & 8 & 10pm & 51.3 & 73.3 \\
\hline $6 / 8 / 2014$ & 10 & 10pm & 42.3 & 60.4 \\
\hline $6 / 9 / 2014$ & 11 & 10pm & 36.2 & 51.7 \\
\hline $6 / 10 / 2014$ & 12 & 10pm & 34.4 & 49.1 \\
\hline $6 / 11 / 2014$ & 13 & 10pm & 31.2 & 44.6 \\
\hline $6 / 12 / 2014$ & 14 & 10pm & 27.7 & 39.6 \\
\hline $6 / 13 / 2014$ & 15 & 10pm & 24.8 & 35.4 \\
\hline $6 / 14 / 2014$ & 16 & 10pm & 22.5 & 32.1 \\
\hline $6 / 15 / 2014$ & 17 & 10pm & 21.1 & 30.1 \\
\hline
\end{tabular}




\section{APPENDIX C: DISSOLVED OXYGEN CONCENTRATION DATA}

\begin{tabular}{|c|c|c|c|c|c|c|}
\hline Date & Time & Time & Temp. & DO & Sat. DO & DO \\
\hline & (Days) & & $\left({ }^{\circ} \mathbf{F}\right)$ & (mg/L) & (mg/L) & (\% Sat) \\
\hline $5 / 29 / 2014$ & 0 & $4 p m$ & 80 & 6.7 & 7.949 & 84.3 \\
\hline $5 / 30 / 2014$ & 1 & 9:30pm & 80 & 7.4 & 7.949 & 93.1 \\
\hline $6 / 1 / 2014$ & 3 & 10pm & 82 & 8.2 & 7.808 & 105.0 \\
\hline $6 / 4 / 2014$ & 6 & 10:30pm & 80 & 7.6 & 7.949 & 95.6 \\
\hline $6 / 6 / 2014$ & 8 & 11:30pm & 78 & 8.9 & 8.094 & 110.0 \\
\hline $6 / 8 / 2014$ & 10 & $10 p m$ & 78 & 7.5 & 8.094 & 92.7 \\
\hline 6/9/2014 & 11 & $11 p m$ & 80 & 8.9 & 7.949 & 112.0 \\
\hline $6 / 10 / 2014$ & 12 & 10:30pm & 79 & 8.4 & 8.094 & 103.8 \\
\hline $6 / 11 / 2014$ & 13 & 10:30pm & 83 & 8.1 & 7.808 & 103.7 \\
\hline $6 / 12 / 2014$ & 14 & $10 p m$ & 81 & 7.4 & 7.949 & 93.1 \\
\hline $6 / 13 / 2014$ & 15 & 10pm & 79 & 8.4 & 8.094 & 103.8 \\
\hline $6 / 14 / 2014$ & 16 & $10: 30$ & 80 & 8.5 & 7.949 & 106.9 \\
\hline $6 / 15 / 2014$ & 17 & 10pm & 80 & 8.4 & 7.949 & 105.7 \\
\hline
\end{tabular}




\section{APPENDIX D: FLUID DYNAMICS AND MASS TRANSFER}

CHARACTERIZATION DATA

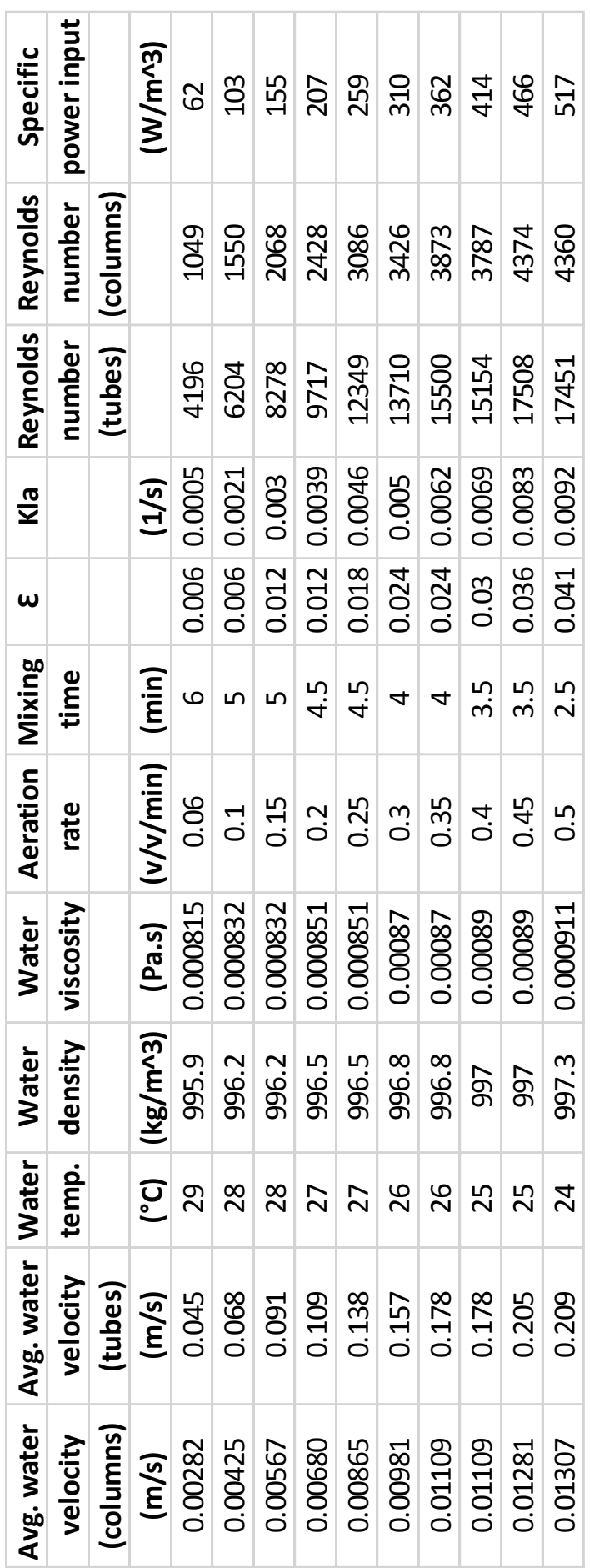




\section{APPENDIX E: CALCULATIONS FOR ALMERIA CENTRIFUGAL PUMP POWER INPUT}

\begin{tabular}{|l|r|}
\hline Parameter & Value \\
\hline Unit \\
\hline Colume roughness/tube diameter & 0.02 \\
\hline Reynolds number (tubes) & 0.004 \\
\hline Reynolds (columns) & 111981 \\
\hline Fanning friction factor for tube & 25196 \\
\hline Fanning friction factor for column & 0.03 \\
\hline Friction loss in the straight tube & 0.008 \\
\hline Friction loss in the straight column & $216 \mathrm{~J} / \mathrm{kg}$ \\
\hline Friction loss in the elbows & $0.000 \mathrm{~J} / \mathrm{kg}$ \\
\hline Friction loss from contraction & $11.239 \mathrm{~J} / \mathrm{kg}$ \\
\hline Friction loss from expansion & $0.211 \mathrm{~J} / \mathrm{kg}$ \\
\hline Total frictional loss & $0.365 \mathrm{~J} / \mathrm{kg}$ \\
\hline Shaft work & $228 \mathrm{~J} / \mathrm{kg}$ \\
\hline Pump efficiency & $228 \mathrm{~J} / \mathrm{kg}$ \\
\hline Pump work & 0.75 \\
\hline Average mass flow rate & $303.75 \mathrm{~J} / \mathrm{kg}$ \\
\hline Pump Wattage & $5.69 \mathrm{~kg} / \mathrm{s}$ \\
\hline Culture volume = & $1728 \mathrm{~W}$ \\
\hline Specific Power input = & $3 \mathrm{~m} 3$ \\
\hline & $13823 \mathrm{Wh} / \mathrm{m}^{3}$ \\
\hline & $13.8 \mathrm{kWh} / \mathrm{m}^{3}$ \\
\hline
\end{tabular}




\section{APPENDIX F: HEAD LOSS CALCULATIONS FOR PROJECTED 3,000-L CAL POLY PBR}

\begin{tabular}{|c|c|c|}
\hline Parameter & Value & Unit \\
\hline Tube roughness/tube diameter $=$ & 0.02 & \\
\hline Column roughness/column diameter $=$ & 0.004 & \\
\hline Reynolds number (tubes) = & 18702 & \\
\hline Reynolds (columns) = & 3547 & \\
\hline Fanning friction factor for tube $=$ & 0.014 & \\
\hline Fanning friction factor for column $=$ & 0.011 & \\
\hline Friction loss in the straight tube $=$ & 5.136 & $\mathrm{~J} / \mathrm{kg}$ \\
\hline Friction loss in the straight column $=$ & 0.00002 & $\mathrm{~J} / \mathrm{kg}$ \\
\hline Friction loss in the elbows $=$ & 2.638 & $\mathrm{~J} / \mathrm{kg}$ \\
\hline Friction loss from contraction $=$ & 0.008 & $\mathrm{~J} / \mathrm{kg}$ \\
\hline Friction loss from expansion $=$ & 0.015 & $\mathrm{~J} / \mathrm{kg}$ \\
\hline Total frictional loss $=$ & 7.80 & $\mathrm{~J} / \mathrm{kg}$ \\
\hline Discharge pressure $=$ & 7750 & $\mathrm{~Pa}$ \\
\hline Head $=$ & 0.79 & $\mathrm{~m}$ \\
\hline
\end{tabular}




\section{APPENDIX G: DISSOLVED OXYGEN PROFILES AT 0.06 V/V/MIN AERATION RATE}
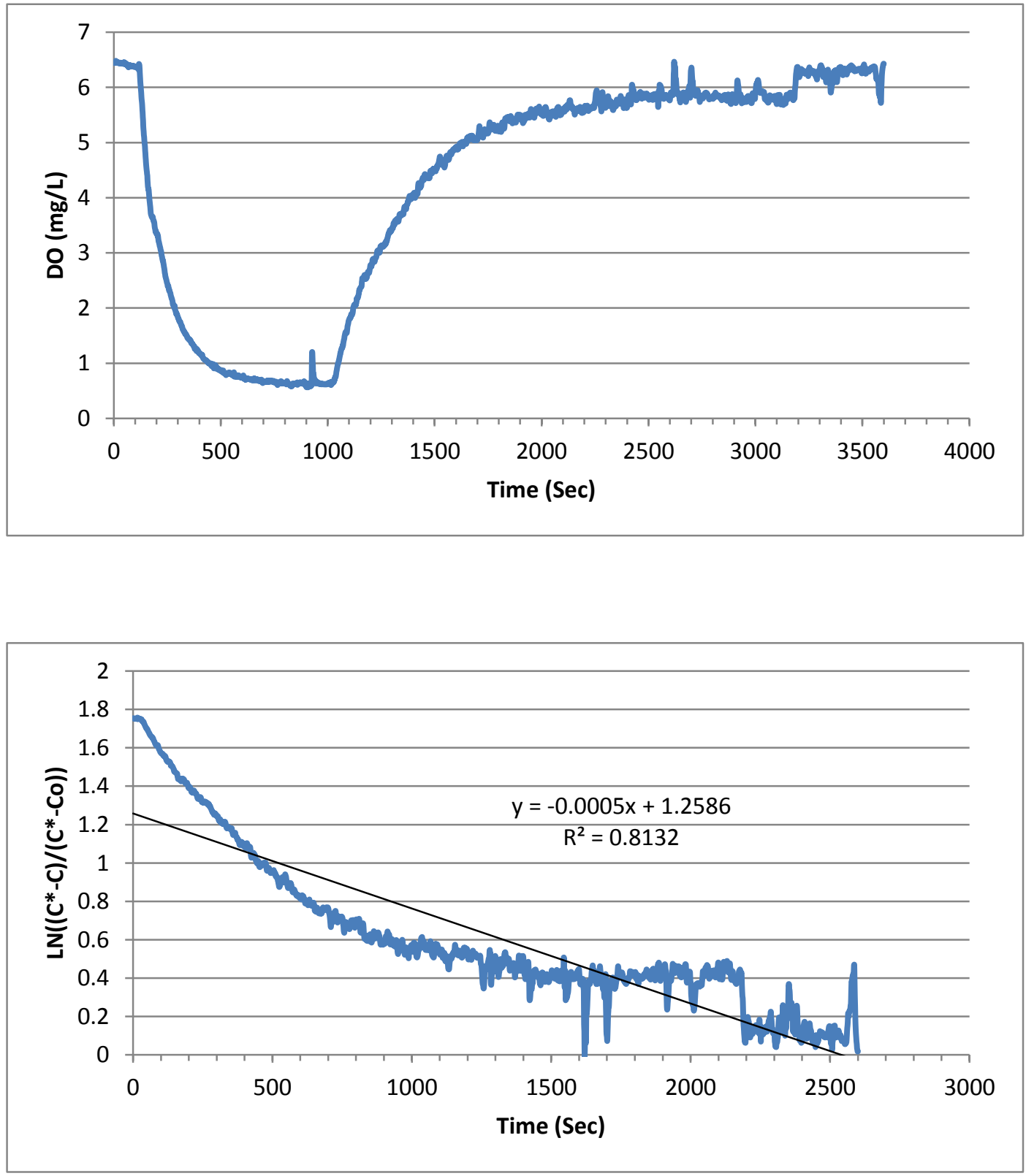


\section{APPENDIX H: DISSOLVED OXYGEN PROFILES AT 0.10 V/V/MIN AERATION RATE}
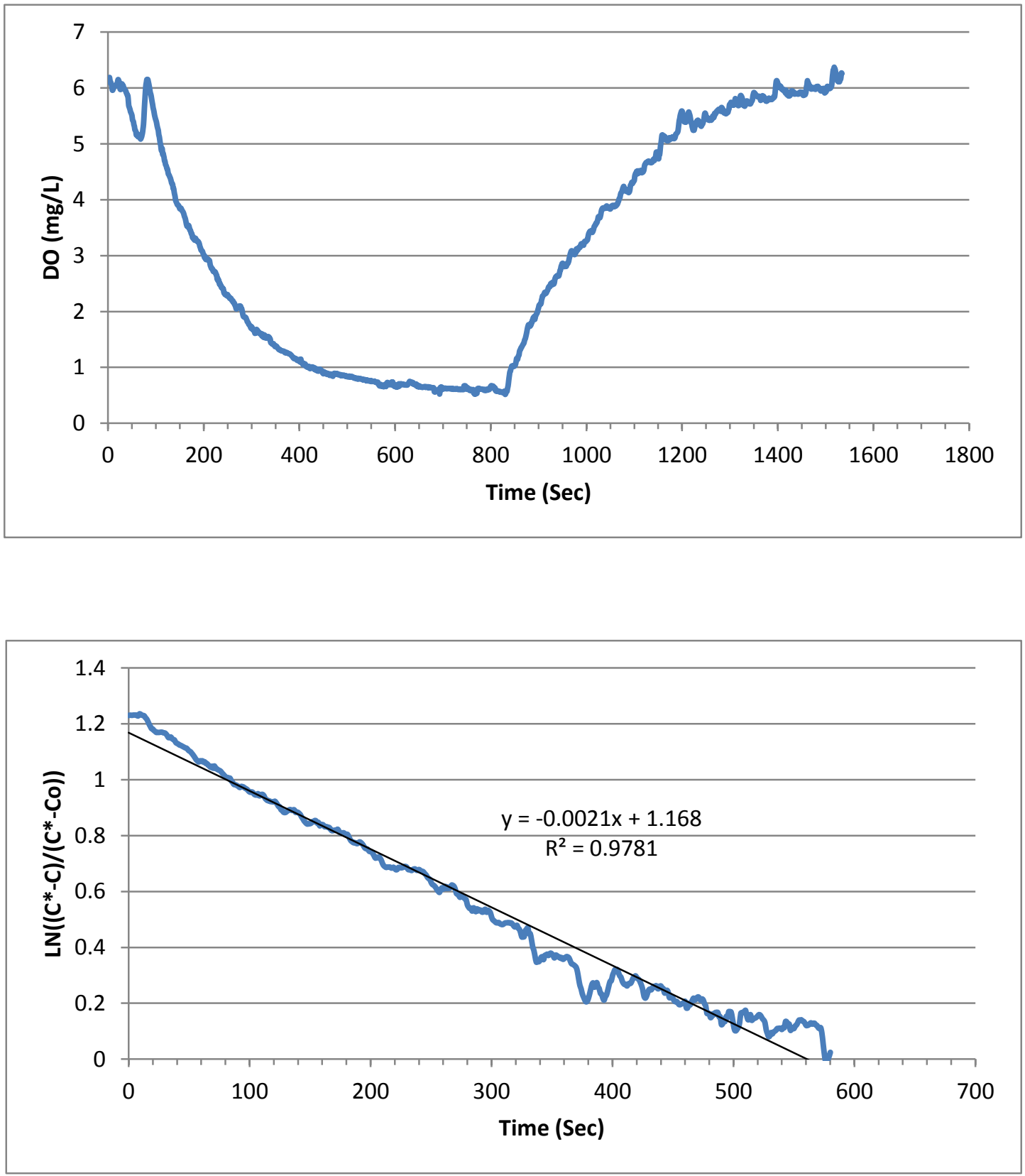


\section{APPENDIX I: DISSOLVED OXYGEN PROFILES AT 0.15 V/V/MIN AERATION RATE}
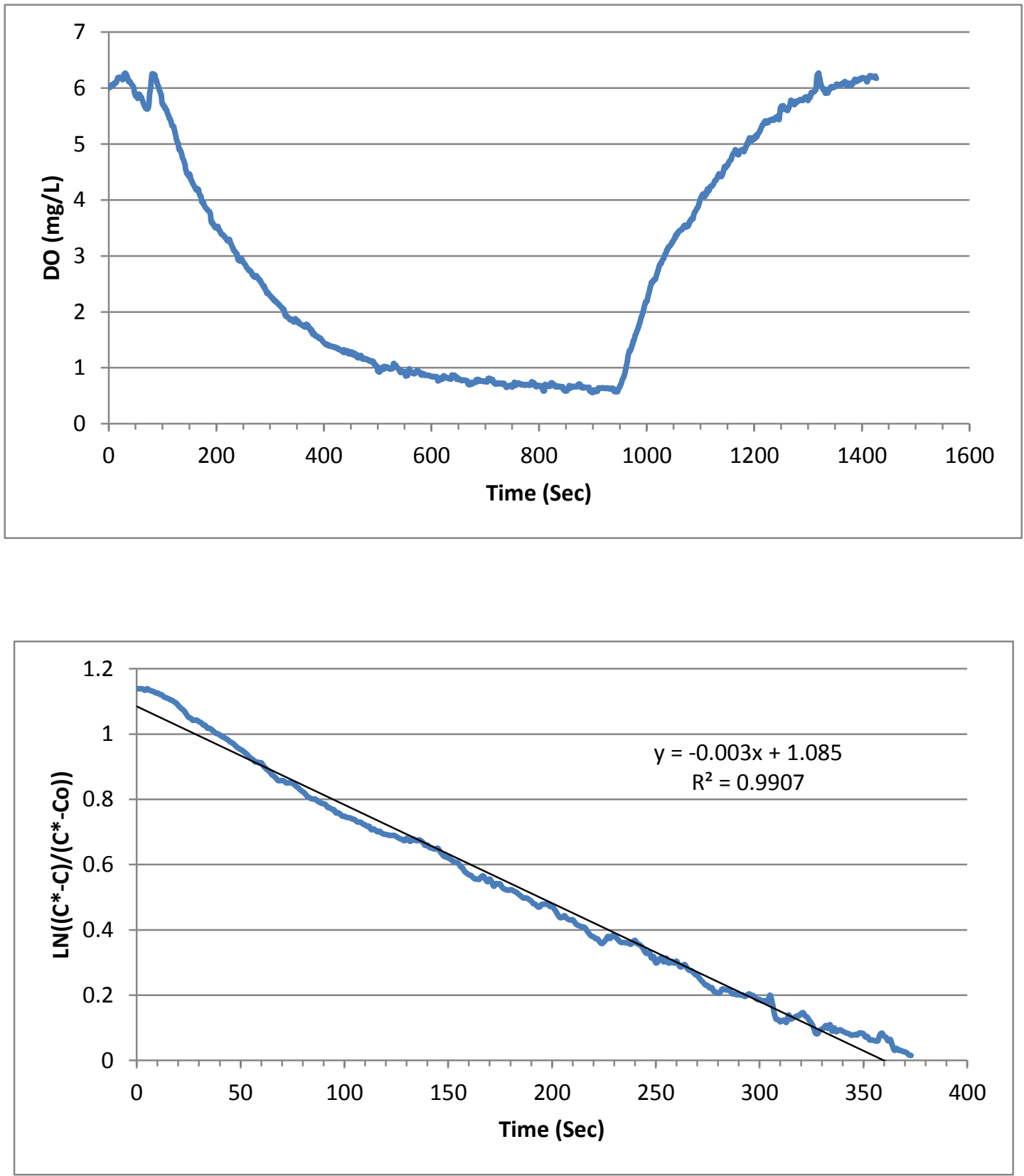


\section{APPENDIX J: DISSOLVED OXYGEN PROFILES AT 0.20 V/V/MIN AERATION RATE}
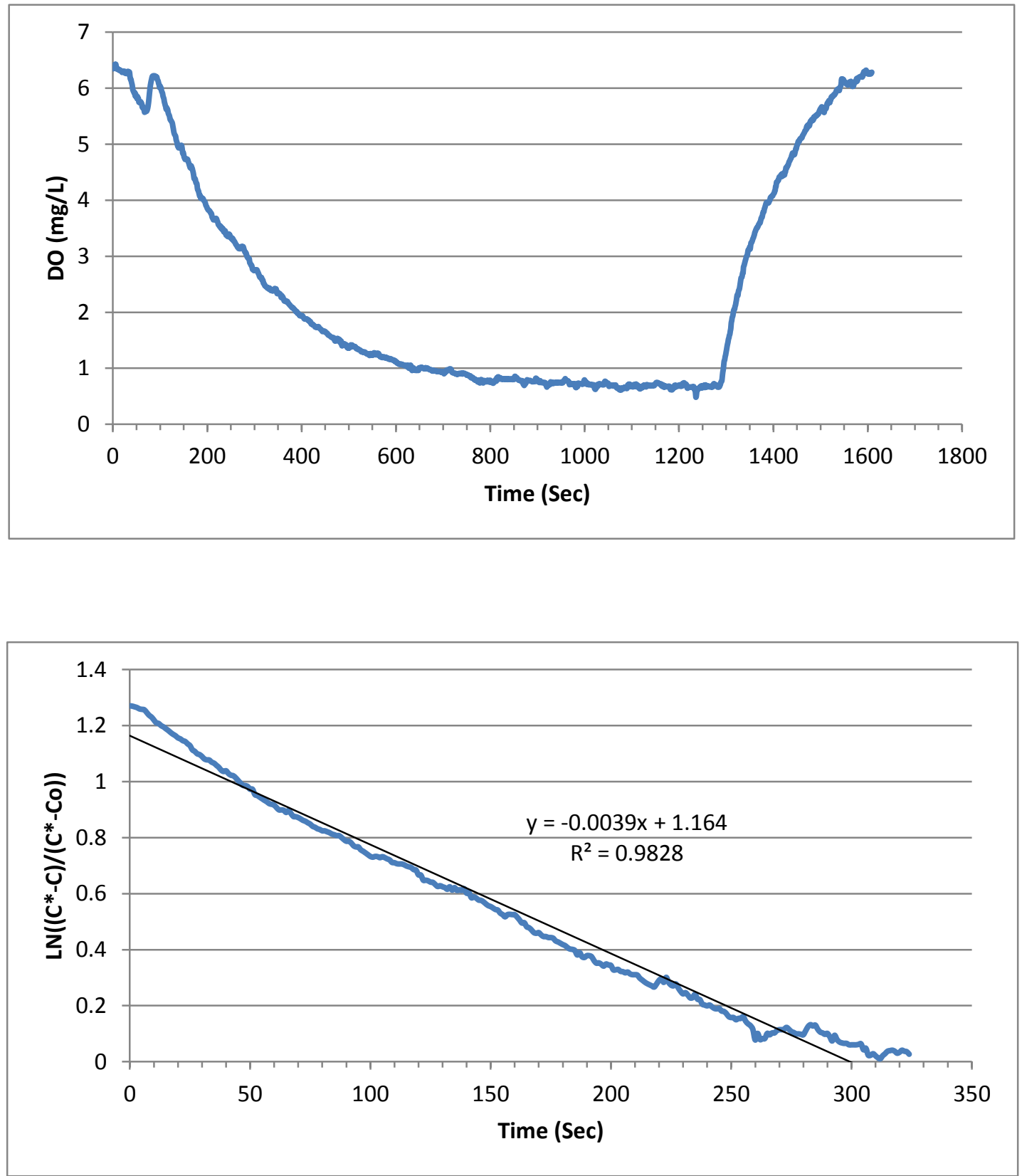


\section{APPENDIX K: DISSOLVED OXYGEN PROFILES AT 0.25 V/V/MIN AERATION RATE}
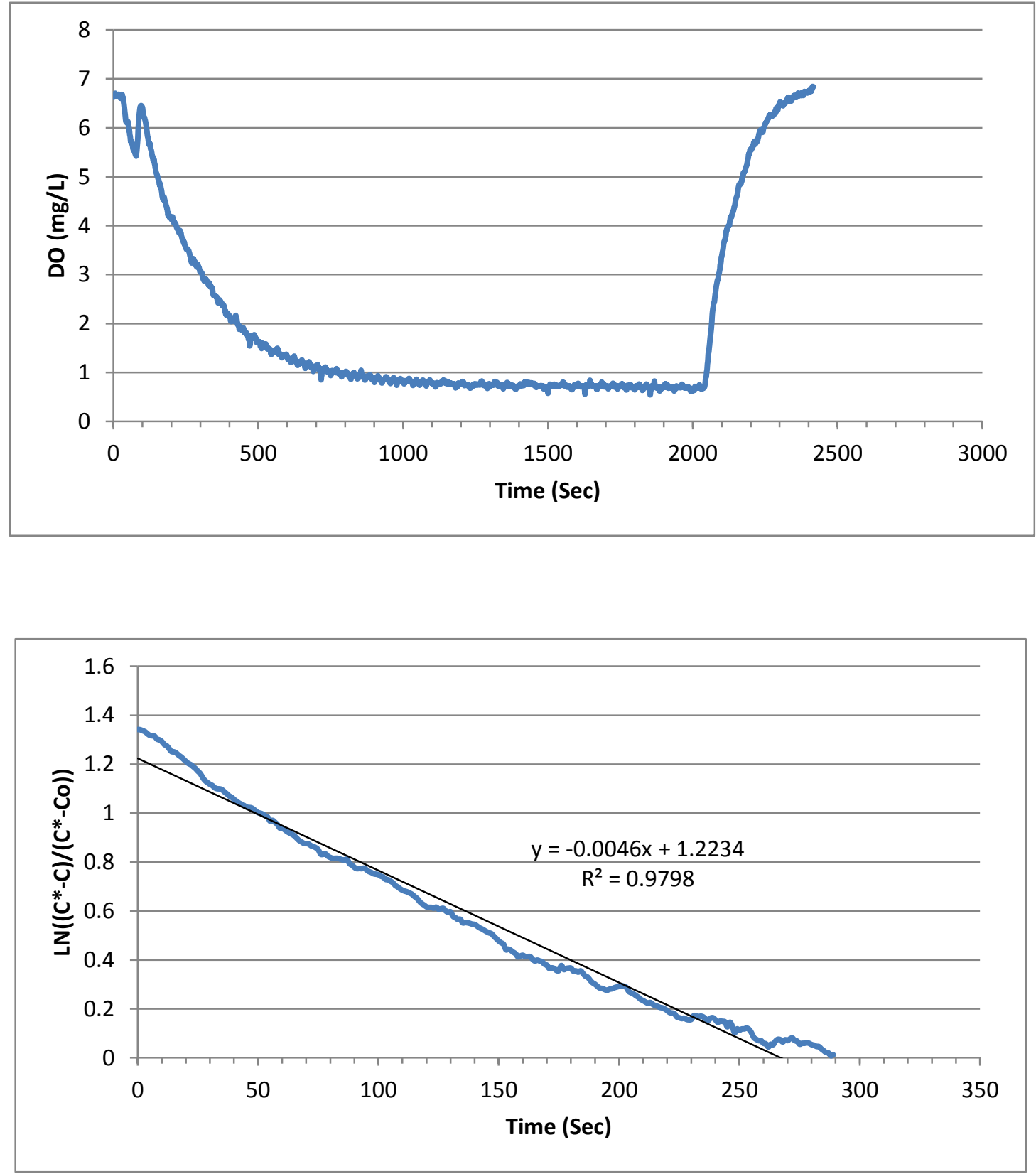


\section{APPENDIX L: DISSOLVED OXYGEN PROFILES AT 0.30 V/V/MIN AERATION RATE}
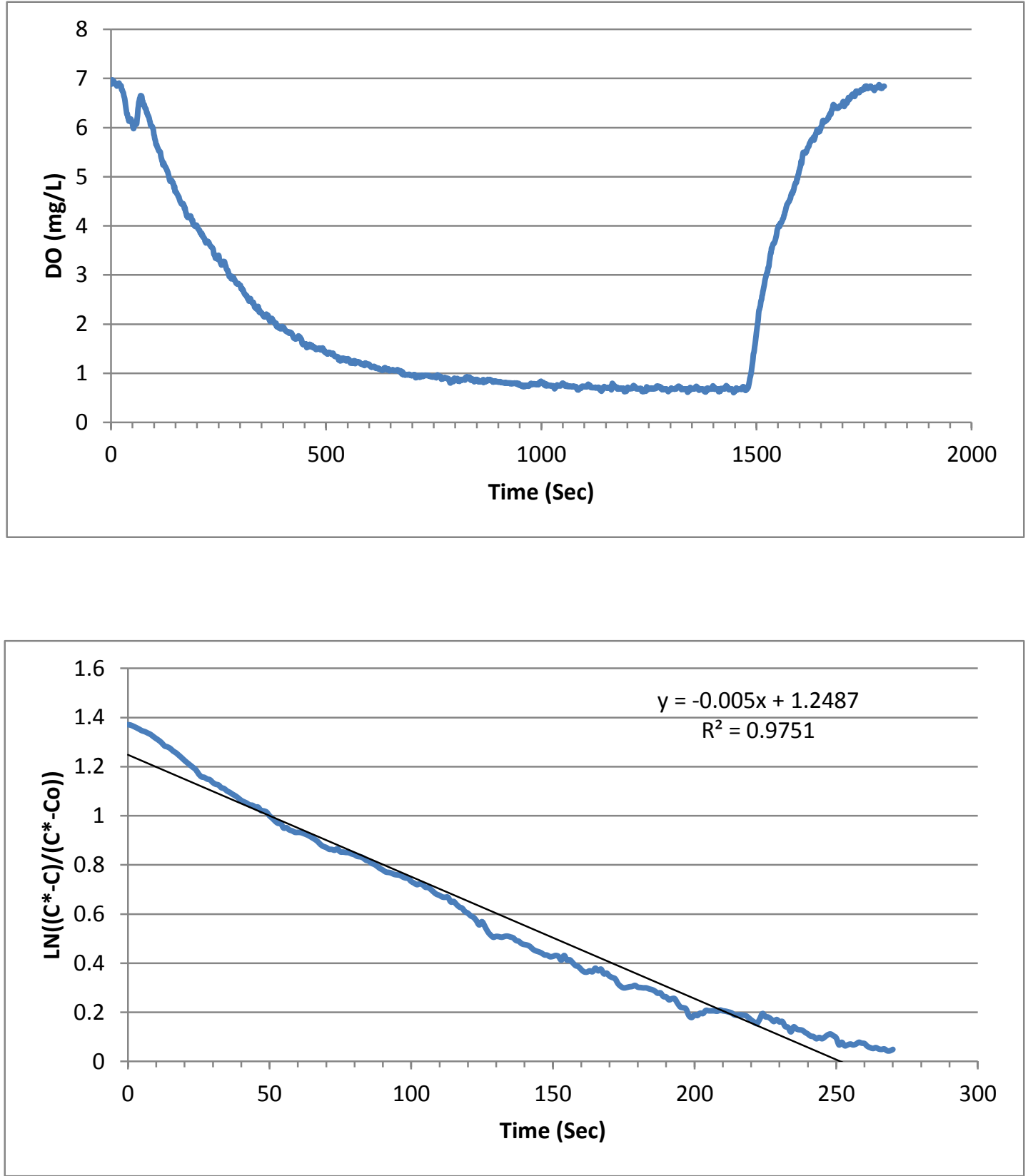


\section{APPENDIX M: DISSOLVED OXYGEN PROFILES AT 0.35 V/V/MIN}

AERATION RATE
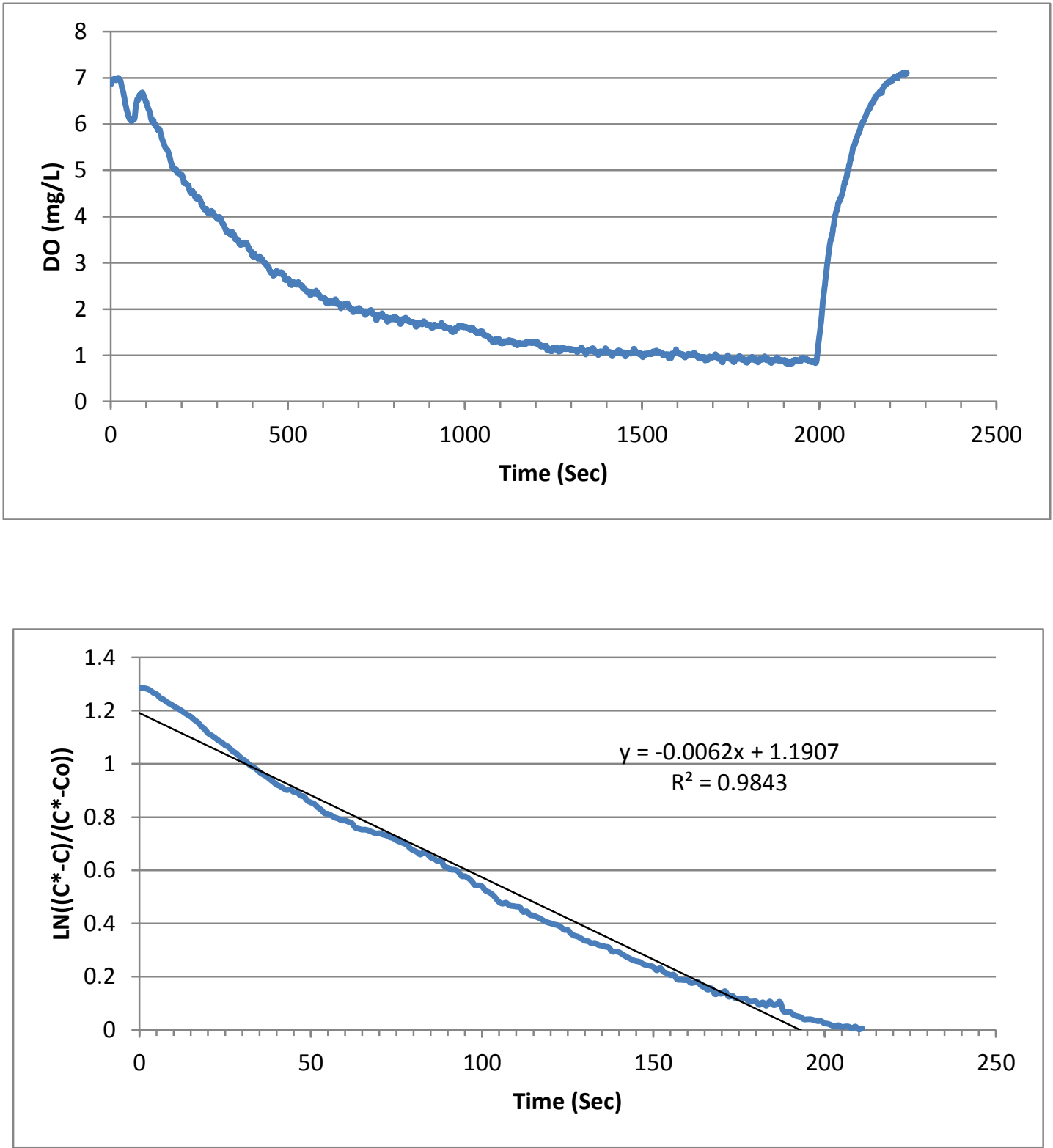


\section{APPENDIX N: DISSOLVED OXYGEN PROFILES AT 0.40 V/V/MIN AERATION RATE}
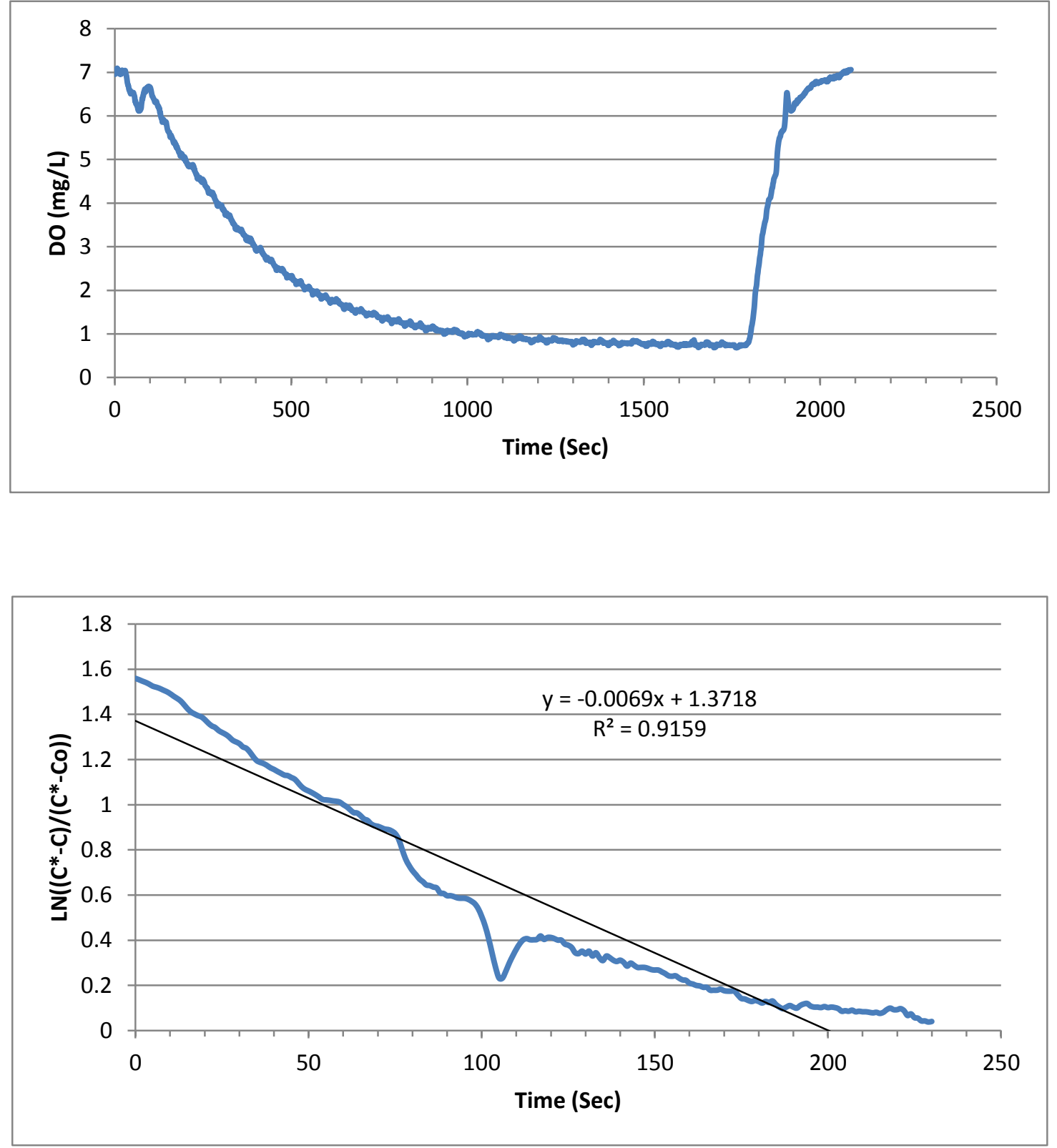


\section{APPENDIX O: DISSOLVED OXYGEN PROFILES AT 0.45 V/V/MIN AERATION RATE}
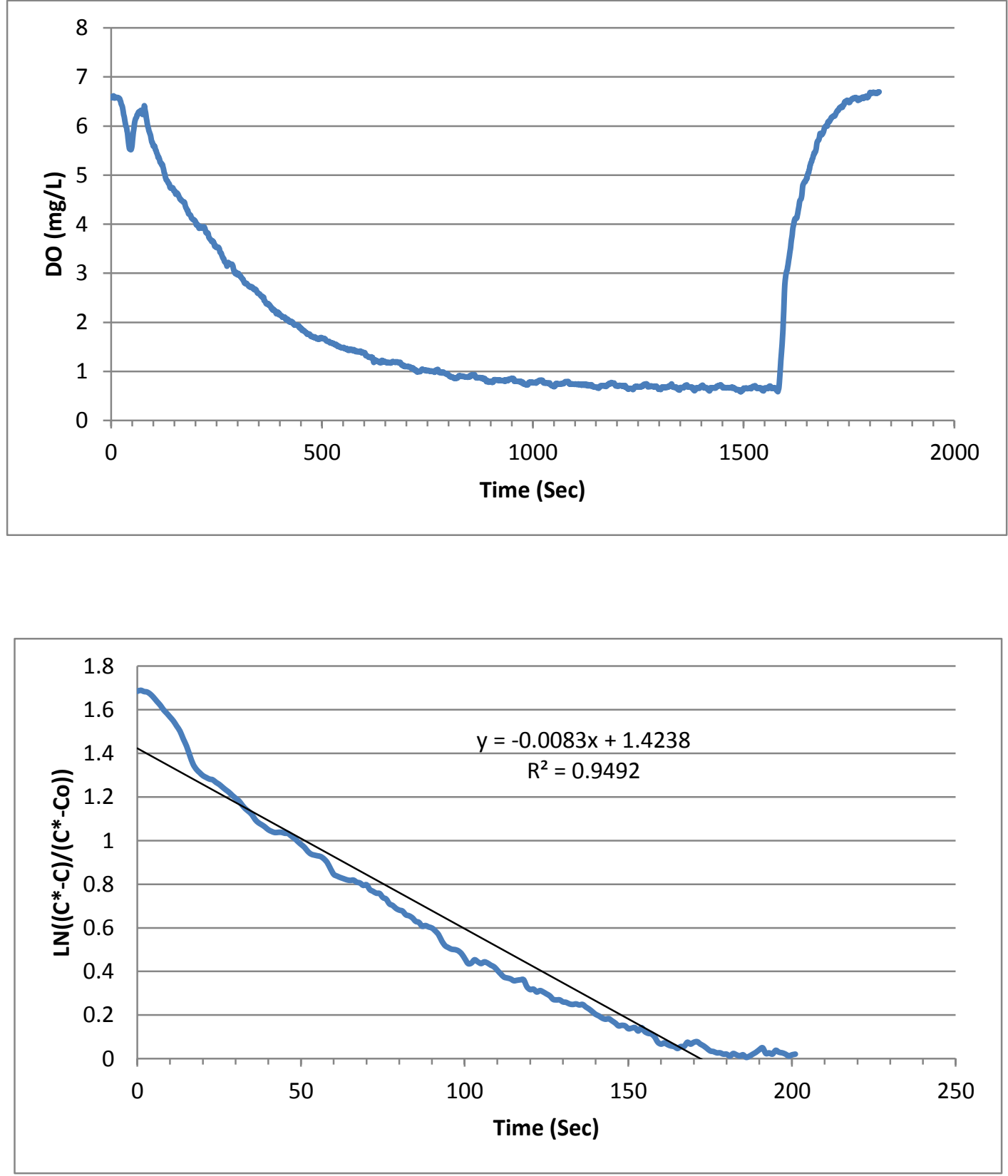


\section{APPENDIX P: DISSOLVED OXYGEN PROFILES AT 0.50 V/V/MIN AERATION RATE}
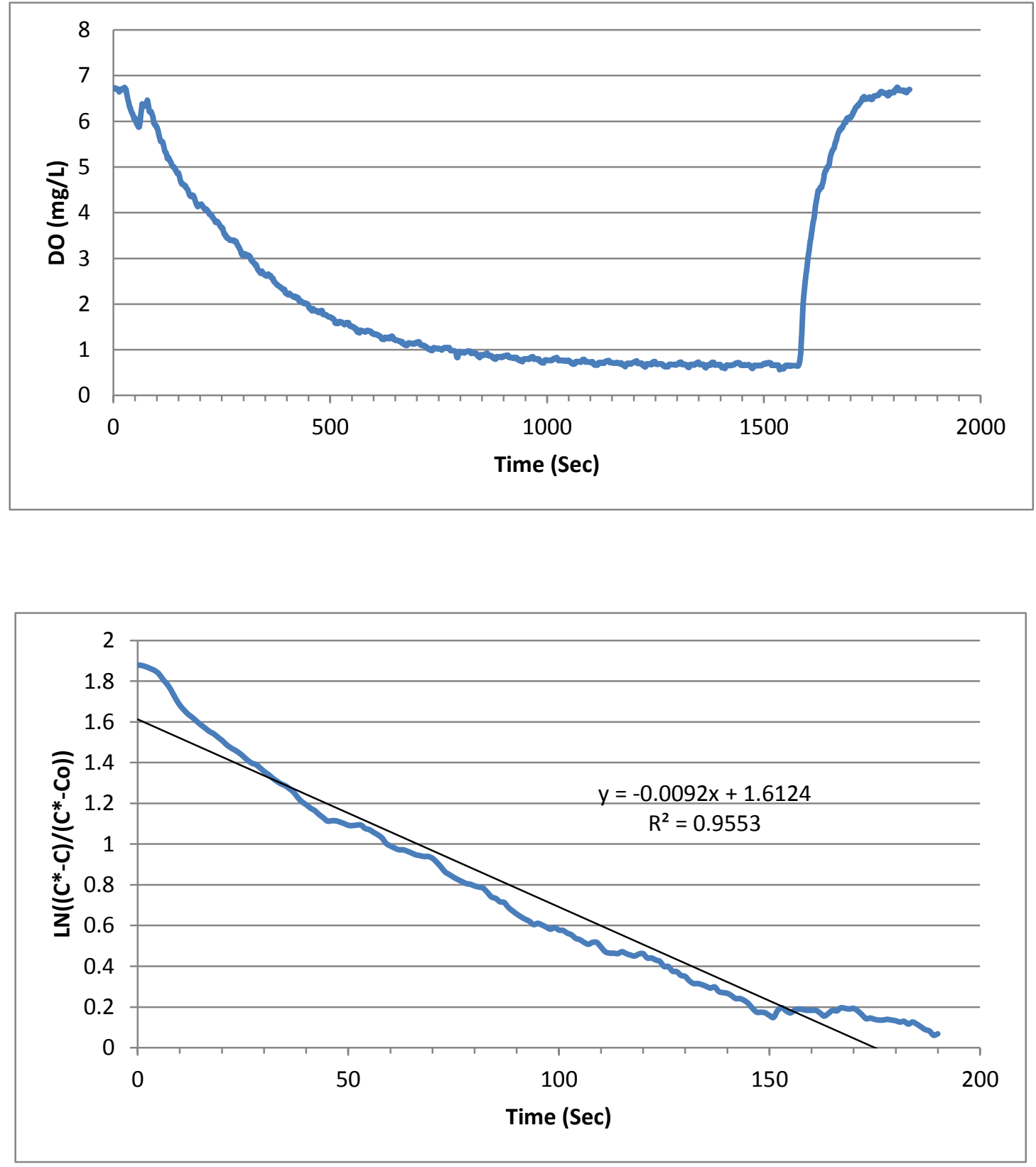


\section{APPENDIX Q: BIOMASS PRODUCTIVITY CALCULATIONS}

\section{Calculations for 3,000-L Cal Poly PBR unit}

$\mu_{\text {net }}=0.5917 \mathrm{~d}^{-1}$

$\Delta \mathrm{X}=\mathrm{X}-\mathrm{Xo}, \quad$ where $\mathrm{X}=1.0373 \mathrm{~g} \mathrm{~L}^{-1}$ at Day 8 of Batch Run \#2

$$
\mathrm{Xo}=0.1 \mathrm{~g} \mathrm{~L}^{-1} \text { at Day } 12 \text { of Batch Run \#2 }
$$

Illuminated surface area (photo-stage) of 3,000-L Cal Poly PBR $=115 \mathrm{~m}^{2}$

Total land area (including empty space) of Prototype PBR $=1.16 \mathrm{~m}^{2}$

Working volume of Prototype PBR $=235 \mathrm{~L}$

$V P=0.5917 \frac{1}{d a y} \cdot\left[\frac{1.0373 g-0.1 g}{L}\right]=0.55 \frac{g}{L \cdot d a y}$

$I S P=0.55 \frac{g}{L \cdot d a y} \cdot \frac{3,000 L}{115 m^{2}}=14.3 \frac{g}{m^{2} \cdot \text { day }}$

$O A P=0.55 \frac{1}{d a y} \cdot \frac{235 L}{1.16 m^{2}}=111 \frac{g}{m^{2} \cdot d a y}$

Sp. power input of 3,000-L Cal Poly PBR using $1^{\text {st }}$ scale-up method $=8.7 \mathrm{kWh} \mathrm{m}^{-3} \mathrm{~d}^{-1}$

$8.7 \frac{\mathrm{kW} \cdot \mathrm{h}}{\mathrm{m}^{3} \cdot \mathrm{day}} \cdot \frac{1 \mathrm{day}}{24 \mathrm{~h}} \cdot \frac{3 \mathrm{~m}^{3}}{1 \mathrm{~kW}} \cdot 1000 \mathrm{~W}=1,088 \mathrm{~W}=1,088 \frac{\mathrm{J}}{\mathrm{s}}$

$0.55 \frac{g}{L \cdot d a y} \cdot 3,000 \mathrm{~L} \cdot \frac{1 \mathrm{~s}}{1,088 \mathrm{~J}} \cdot \frac{1 \mathrm{day}}{86,400 \mathrm{~s}} \cdot \frac{1,000 \mathrm{mg}}{1 \mathrm{~g}} \cdot \frac{1,000 \mathrm{~J}}{1 \mathrm{~kJ}}=17.6 \frac{\mathrm{mg}}{\mathrm{kJ}}$ 
Sp. power input of 3,000-L Cal Poly PBR using $2^{\text {st }}$ scale-up method $=8.0 \mathrm{kWh} \mathrm{m}^{-3} \mathrm{~d}^{-1}$

$$
\begin{aligned}
& 8.0 \frac{\mathrm{kW} \cdot h}{\mathrm{~m}^{3} \cdot \mathrm{day}} \cdot \frac{1 \text { day }}{24 \mathrm{~h}} \cdot \frac{3 \mathrm{~m}^{3}}{1 \mathrm{~kW}} \cdot 1000 \mathrm{~W}=1,000 \mathrm{~W}=1,000 \frac{\mathrm{J}}{\mathrm{s}} \\
& 0.55 \frac{\mathrm{g}}{\mathrm{L} \cdot \mathrm{day}} \cdot 3,000 \mathrm{~L} \cdot \frac{1 \mathrm{~s}}{1,000 \mathrm{~J}} \cdot \frac{1 \mathrm{day}}{86,400 \mathrm{~s}} \cdot \frac{1,000 \mathrm{mg}}{1 \mathrm{~g}} \cdot \frac{1,000 \mathrm{~J}}{1 \mathrm{~kJ}}=19.1 \frac{\mathrm{mg}}{\mathrm{kJ}}
\end{aligned}
$$

\section{Calculations for 3,000-L Almeria PBR unit}

Illuminated surface area (photo-stage) $=110 \mathrm{~m}^{2}$

Total land area (including empty space) $=40 \mathrm{~m}^{2}$

Working volume $=3,000 \mathrm{~L}$

$$
\begin{aligned}
& I S P=0.42 \frac{g}{L \cdot d a y} \cdot \frac{3,000 L}{110 m^{2}}=11.5 \frac{g}{m^{2} \cdot d a y} \\
& O A P=0.42 \frac{g}{L \cdot d a y} \cdot \frac{3,000 L}{40 m^{2}}=31.5 \frac{g}{m^{2} \cdot \text { day }}
\end{aligned}
$$

Sp. power input of 3,000-L Almeria PBR from lierature review $=11 \mathrm{kWh} \mathrm{m}^{-3} \mathrm{~d}^{-1}$

$$
\begin{aligned}
& 11 \frac{\mathrm{kW} \cdot \mathrm{h}}{\mathrm{m}^{3} \cdot \mathrm{day}} \cdot \frac{1 \mathrm{day}}{24 \mathrm{~h}} \cdot \frac{3 \mathrm{~m}^{3}}{1 \mathrm{~kW}} \cdot 1000 \mathrm{~W}=1,375 \mathrm{~W}=1,375 \frac{\mathrm{J}}{\mathrm{s}} \\
& 0.42 \frac{\mathrm{g}}{\mathrm{L} \cdot \text { day }} \cdot 3,000 \mathrm{~L} \cdot \frac{1 \mathrm{~s}}{1,375 \mathrm{~J}} \cdot \frac{1 \mathrm{day}}{86,400 \mathrm{~s}} \cdot \frac{1,000 \mathrm{mg}}{1 \mathrm{~g}} \cdot \frac{1,000 \mathrm{~J}}{1 \mathrm{~kJ}}=10.6 \frac{\mathrm{mg}}{\mathrm{kJ}}
\end{aligned}
$$

Sp. power input of 3,000-L Almeria PBR from calculated values $=20 \mathrm{kWh} \mathrm{m}^{-3} \mathrm{~d}^{-1}$

$$
\begin{aligned}
& 20 \frac{\mathrm{kW} \cdot \mathrm{h}}{\mathrm{m}^{3} \cdot \text { day }} \cdot \frac{1 \text { day }}{24 \mathrm{~h}} \cdot \frac{3 \mathrm{~m}^{3}}{1 \mathrm{~kW}} \cdot 1000 \mathrm{~W}=2,500 \mathrm{~W}=2,500 \frac{\mathrm{J}}{\mathrm{s}} \\
& 0.42 \frac{\mathrm{g}}{\mathrm{L} \cdot \text { day }} \cdot 3,000 \mathrm{~L} \cdot \frac{1 \mathrm{~s}}{2,500 \mathrm{~J}} \cdot \frac{1 \text { day }}{86,400 \mathrm{~s}} \cdot \frac{1,000 \mathrm{mg}}{1 \mathrm{~g}} \cdot \frac{1,000 \mathrm{~J}}{1 \mathrm{~kJ}}=5.8 \frac{\mathrm{mg}}{\mathrm{kJ}} \\
& 162
\end{aligned}
$$




\section{APPENDIX R: AIRLIFT CALCULATIONS FOR THE 3,000-L CAL POLY PBR}

Pump work $=\frac{\text { shaft } \text { work }}{\text { efficiency }}=\frac{0.25 \mathrm{~kW}}{0.25}=1 \mathrm{~kW}$

Specific power input $=\frac{1 \mathrm{~kW}}{3 \mathrm{~m}^{3}} \cdot \frac{24 \mathrm{hrs}}{1 \mathrm{day}} \cdot 1$ day $=8 \frac{\mathrm{kWh}}{\mathrm{m}^{3} \mathrm{day}}$ 